Portland State University

PDXScholar

Summer 8-9-2019

\title{
Assessment of Cluster Chondrite Accretion \\ Temperature Using Electron Backscatter Diffraction and Implications for Chondrule Formation Models
}

Secana Portia Goudy

Portland State University

Follow this and additional works at: https://pdxscholar.library.pdx.edu/open_access_etds

Part of the Geology Commons

Let us know how access to this document benefits you.

\section{Recommended Citation}

Goudy, Secana Portia, "Assessment of Cluster Chondrite Accretion Temperature Using Electron Backscatter Diffraction and Implications for Chondrule Formation Models" (2019). Dissertations and Theses. Paper 5268.

https://doi.org/10.15760/etd.7141

This Thesis is brought to you for free and open access. It has been accepted for inclusion in Dissertations and Theses by an authorized administrator of PDXScholar. Please contact us if we can make this document more accessible: pdxscholar@pdx.edu. 


\title{
Assessment of Cluster Chondrite Accretion Temperature Using Electron Backscatter Diffraction and Implications for Chondrule Formation Models
}

\author{
by
}

Secana Portia Goudy

A thesis submitted in partial fulfillment of the requirements for the degree of

\author{
Master of Science \\ in \\ Geology
}

\section{Thesis Committee:}

Alexander Ruzicka, Chair

Richard Hugo

Nancy Price

Portland State University
2019 
C2019 Secana Portia Goudy 


\begin{abstract}
This thesis studies ordinary chondrites with cluster chondrite lithologies using electron backscatter diffraction so as to measure the temperatures of their olivine grains during deformation, for the purpose of constraining the accretion temperatures of cluster chondrites and creating new constraints on chondrule formation models. Samples analyzed with the technique are shock classified in this thesis as S1 and are type 3, so the deformation analyzed is interpreted to represent the temperatures of the chondrules during accretional deformation. It is found that the studied samples are primarily composed of chondrules at hot temperatures $\left(>850^{\circ} \mathrm{C}\right)$ during deformation, mixed with a questionable minority at cold $\left(<850^{\circ} \mathrm{C}\right)$ temperatures. This is interpreted to represent a primarily hot or possibly heterogeneous temperature of accretion; the objects accreting were mostly hot chondrules with a possible addition of cold chondrules. This interpretation establishes two new possible constraints for chondrule formation models, requiring that they must allow for chondrule accretion shortly after the heating event and that they might require the mixing of hot and cold chondrules in the short time period prior to that accretion. The former of these new constraints has much stronger evidence for it than the latter. These new constraints are most compatible with established protoplanetary bow shock and impact formation models, though if the mixing constraint can be dismissed density shockwave models are also viable. Other models are either wholly incompatible with the new constraints or require modification to be consistent with them.
\end{abstract}




\section{Acknowledgements}

I would like to thank Dr. Alex Ruzicka, for his infectious enthusiasm for meteoritics and introducing me to an endlessly fascinating field of study. I must also thank Dr. Dick Pugh, who is possessed of a similarly infectious passion for meteoritics and for his financial contributions to this research. I would also like to thank Dr. Nancy Price, Dr. Ken Cruikshank, and Veronica Biesiada for their helpful advice in navigating graduate school. I also am compelled to give recognition as a whole to the geology department graduate students of Portland State University, whose comradery created an excellent environment to further the pursuit of my thesis. 
Table of Contents

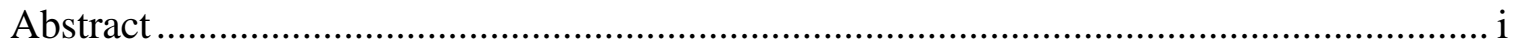

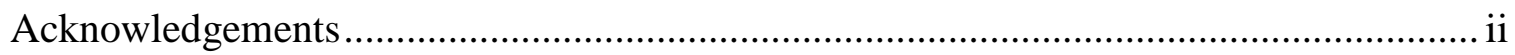

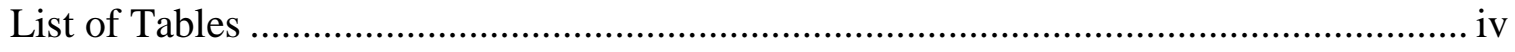

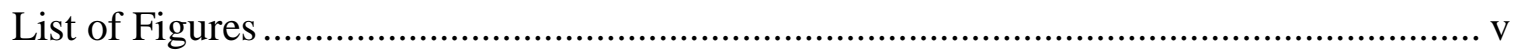

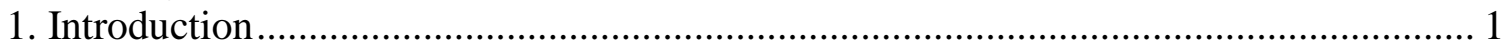

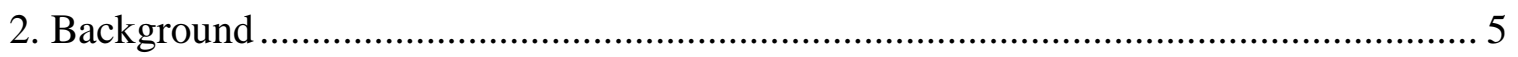

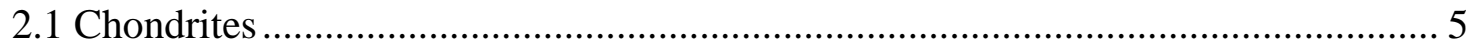

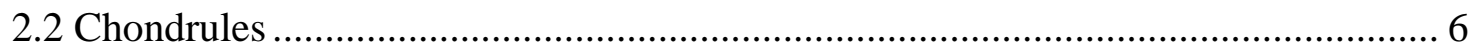

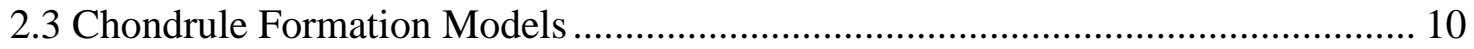

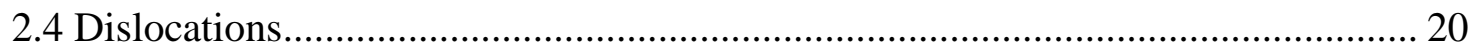

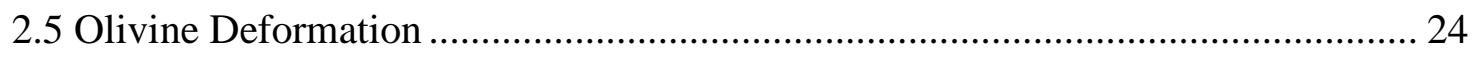

2.6 Electron Backscatter Diffraction...................................................................... 26

2.7 Shock Metamorphism and Postshock Annealing ........................................... 28

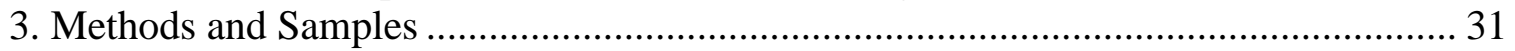

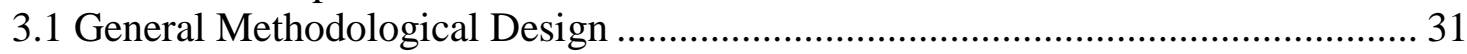

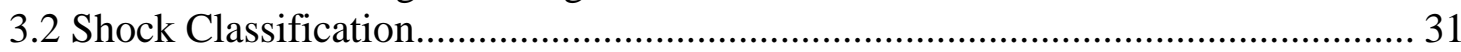

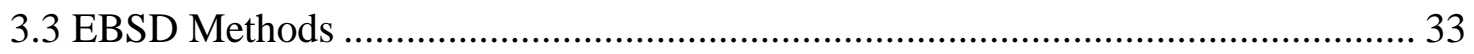

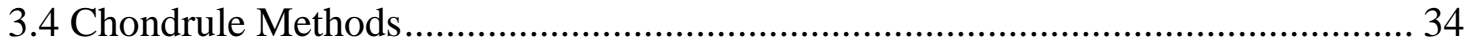

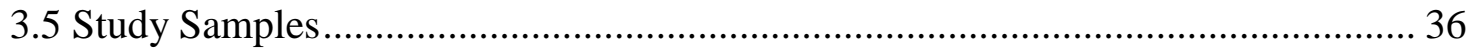

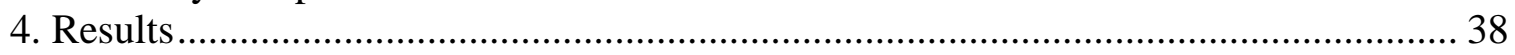

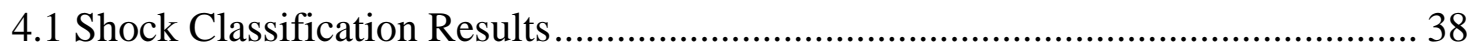

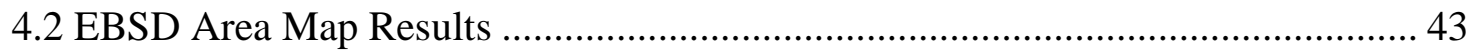

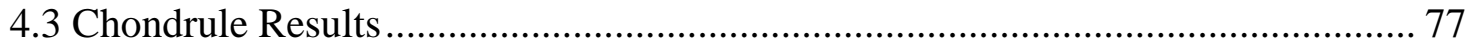

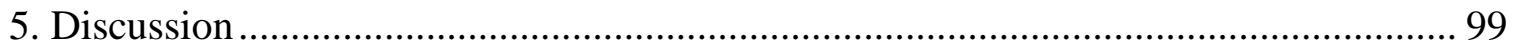

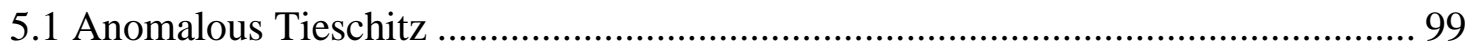

5.2 Relations between Parameters of Deformation and Temperature ...................... 100

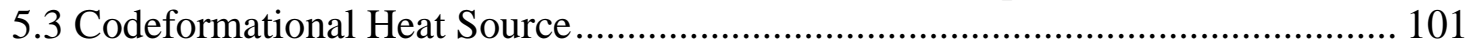

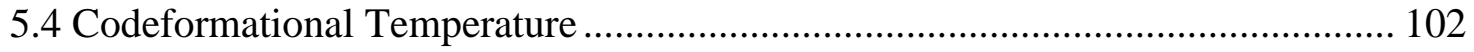

5.5 Implications for Chondrule Formation Models ............................................. 103

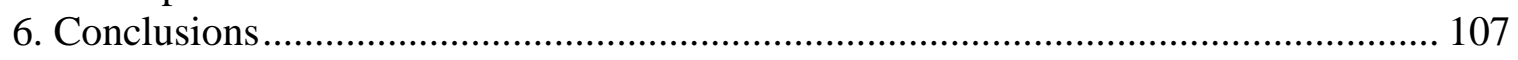

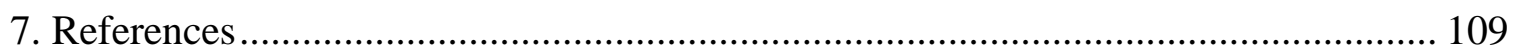

Appendix: Shock Data Figures of Non-EBSD Analyzed Samples ............................ 129 


\section{List of Tables}

Table 1. Summary of Jamsja and Ruzicka (2010) Classification Characteristics 33

Table 2. Table of Samples

Table 3. Olivine Shock Stage Data for Studied Samples.............................................. 38

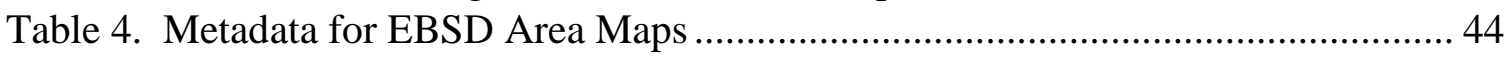

Table 5. EBSD Area Maps Results.......................................................................... 46

Table 6. Apparent Annealing Parameters of EBSD Analyzed Samples ......................... 46

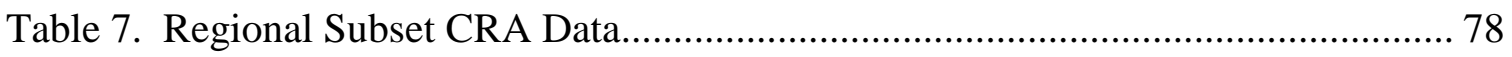

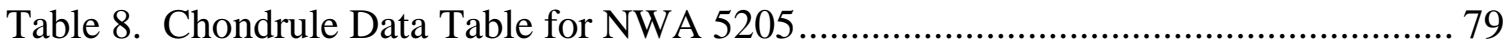

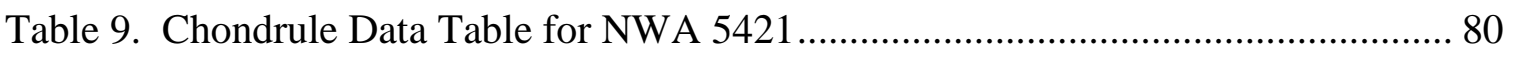

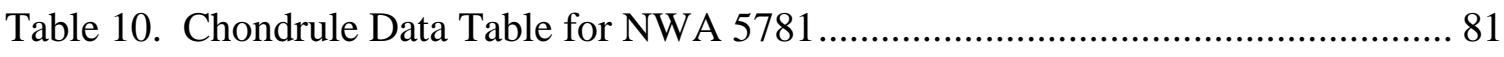

Table 11. Chondrule Data Table for Tieschitz........................................................... 82

Table 12. Summary of Implications on Formation Models for New Constraints ......... 106 


\section{List of Figures}

Figure 1. Example Image of a Cluster Chondrite ……….............................................. 4

Figure 2. Cartoon Diagrams of Edge and Screw Dislocations ....................................... 23

Figure 3. Geometric Relations Among Rotation Axes, Slip Systems, and Subgrain

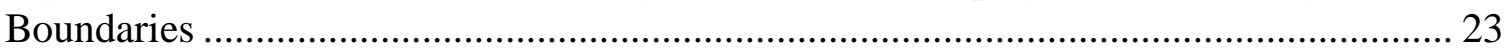

Figure 4. Diagram for Relating Crystallographic Rotation Axis Data to Olivine Slip

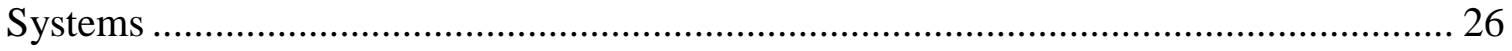

Figure 5. Set Up for EBSD Analysis with SEM ........................................................ 28

Figure 6. Grain Shock Stage Histogram of Sample CML 0954-1A-1 .............................. 39

Figure 7. Grain Shock Stage Histogram of Sample CML 0953-3A ................................. 39

Figure 8. Grain Shock Stage Histogram of Sample CML 0244-2 …………………....... 39

Figure 9. Grain Shock Stage Histogram of Sample CML 0781B .................................... 39

Figure 10. Shock Map of Sample CML 0954-1A-1 ………........................................ 40

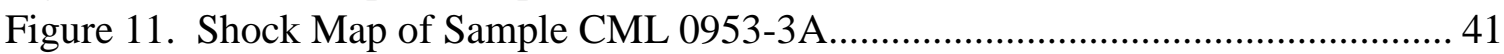

Figure 12. Shock Map of Sample CML 0244-2 ………………………………....... 42

Figure 13. Shock Map of Sample CML 0781B ………………………...................... 42

Figure 14. EBSD Mapped Areas of Selected Samples ..................................................... 45

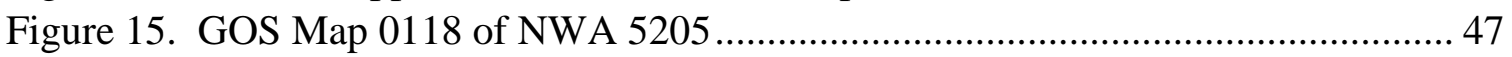

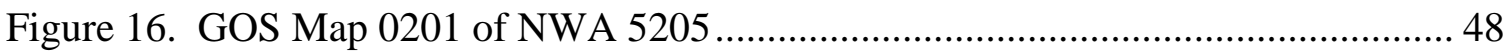

Figure 17. GOS Map 1215 of NWA 5421 ................................................................ 49

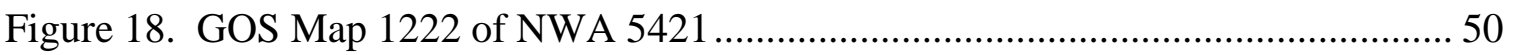

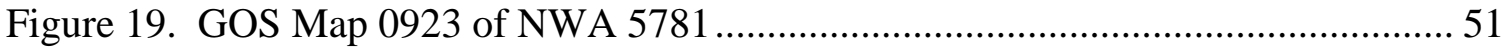

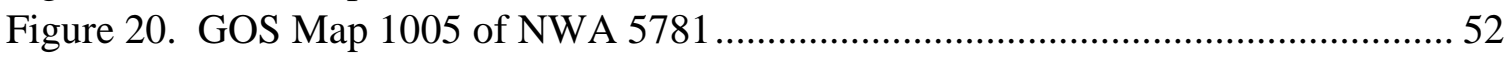

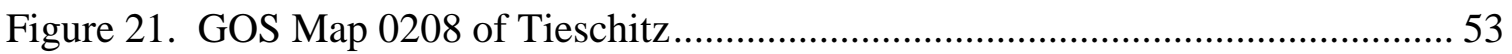

Figure 22. GOS Map 0215 of Tieschitz................................................................. 54

Figure 23. Local Misorientation Map 0118 of NWA 5205 .......................................... 55

Figure 24. Local Misorientation Map 0201 of NWA 5205 ........................................... 56

Figure 25. Local Misorientation Map 1215 of NWA 5421 ........................................... 57

Figure 26. Local Misorientation Map 1222 of NWA 5421 .......................................... 58

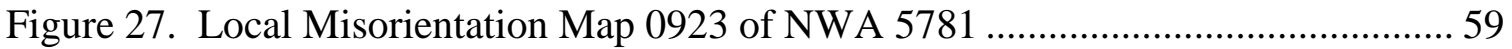

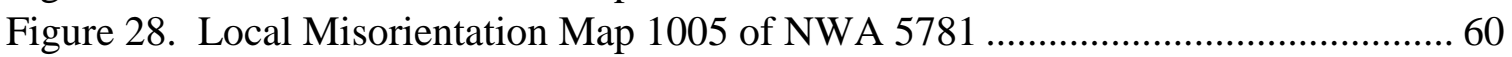

Figure 29. Local Misorientation Map 0208 of Tieschitz ................................................... 61

Figure 30. Local Misorientation Map 0215 of Tieschitz ................................................ 62

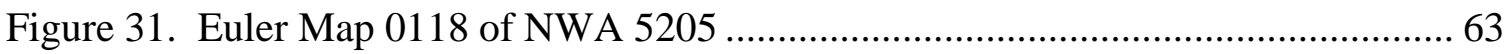

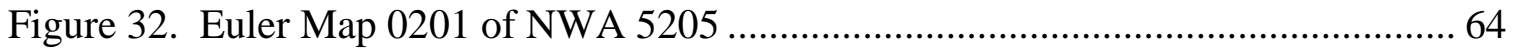

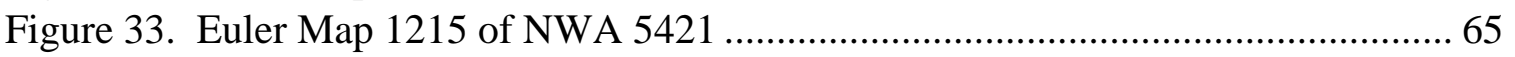

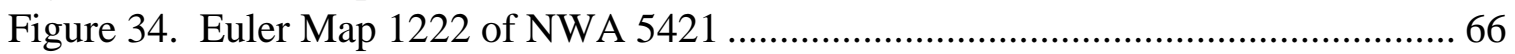

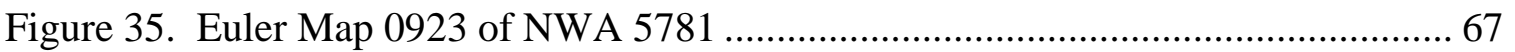

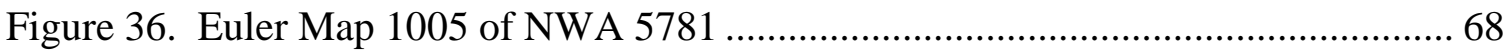

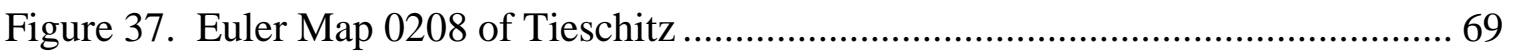

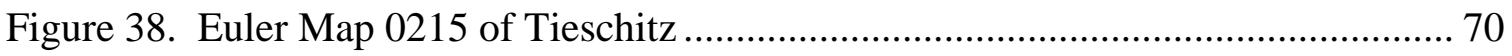




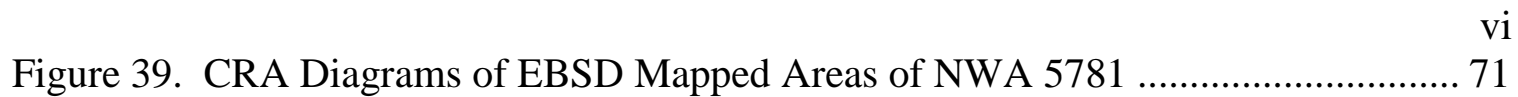

Figure 40. CRA Diagrams of EBSD Mapped Areas of NWA 5421 ............................ 72

Figure 41. CRA Diagrams of EBSD Mapped Areas of NWA 5205 .............................. 73

Figure 42. CRA Diagrams of EBSD Mapped Areas of Tieschitz ................................. 74

Figure 43. Diagrams Comparing GOS and MOS to Weighted Shock Stages ................ 75

Figure 44. Graph Comparing Mean GOS/Median GOS and Temperature Parameters... 76

Figure 45. Ternary Displaying Modal Crystal Rotation Axes in Analyzed Samples ...... 77

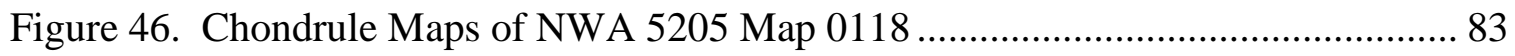

Figure 47. Chondrule Maps of NWA 5205 Map 0201 ................................................. 84

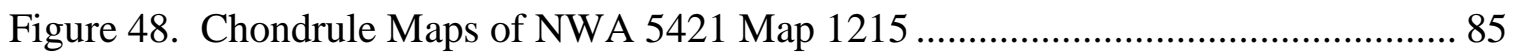

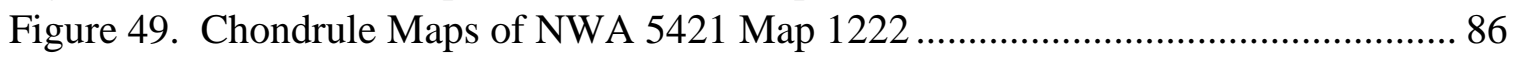

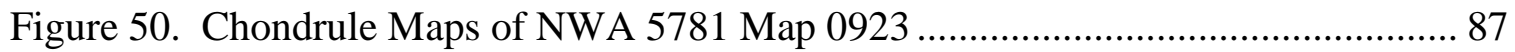

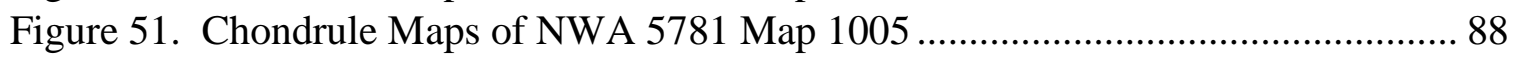

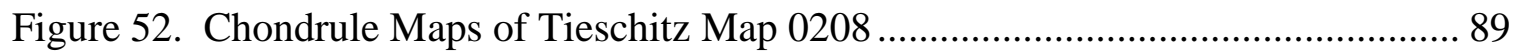

Figure 53. Chondrule Maps of Tieschitz Map 0215 .................................................. 90

Figure 54. Graph of Chondrule Temperature Parameters............................................ 91

Figure 55. Ternary Displaying Chondrule CRA Data of NWA 5205 .......................... 92

Figure 56. Ternary Displaying Chondrule CRA Data of NWA 5421 ........................... 93

Figure 57. Ternary Displaying Chondrule CRA Data of NWA 5781 ............................ 94

Figure 58. Ternary Displaying Chondrule CRA Data of Tieschitz ............................. 95

Figure 59. Graphs Comparing Chondrule Temperature and Shape Parameters ............. 96

Figure 60. Graphs Comparing Chondrule Temperature Parameters and Mean GOS...... 97

Figure 61. Graphs Comparing Chondrule Mean GOS and Shape Parameters ............... 98 


\section{Introduction}

The conventional view on chondrite accretion is that of cold accretion - that the components that came to together to create chondrites were cold during the event. This is based on evidence such as surviving presolar grains and amorphous carbon in unequilibrated ordinary chondrites, which are incompatible with exposure to higher temperatures (Michel-Levy and Lautie, 1981; Rubin and Brearley, 1996). This view is opposed by that of hot accretion: that chondrites' components were hot during accretion. While seemingly incompatible with the evidence for cold accretion, there is evidence possibly indicating hot accretion. Such evidence includes plastic deformation of chondrules in low grade chondrites, as well as an observed "burst" chondrule in Tieschitz (Hutchison et al., 1979).

Cluster chondrites are chondrite clasts with a high chondrule content (88-92 vol\%) that are characterized by close-fit textures of their chondrules and a low content of chondrule fragments and fine grained matrix (Metzler, 2012). Cluster chondrites are defined by their petrographic texture and have no special distinction in their overall chemistry and mineralogy from chondrites in general (Metzler, 2012; Metzler and Pack, 2016). Whether the proportions of chondrule texture types in cluster chondrites differ from those of non-cluster chondrites is inconsistent in the literature. Metzler (2012) reports no difference while Holmén and Wood (1986) observe overabundant granular and porphyritic chondrules, and underabundant barred and radiating chondrules. Chondrules in cluster chondrites exhibit a high degree of apparent deformation, based on a high proportion of irregular shapes, compared to non-cluster chondrites (Metzler, 2012). 
Cluster chondrites may be formed by the accretion and plastic deformation of hot chondrules (Hutchison et al., 1979; Hutchison and Bevan, 1983; Holmén and Wood, 1986; Sanders and Hill, 1994; Hutchison, 1996b; Metzler, 2012). However, it is also possible that they may have formed by aggregation of hot chondrules into a clump in the solar nebula (Rubin and Brearley, 1996). It has been estimated by their thermal histories that chondrules could have remained plastically deformable for 0.3-60 hours after their heating (Hood and Ciesla, 2001; Desch et al., 2012). Other competing explanations for close-fit textures, such as pressure solution, have been advocated by other workers. This mechanism would work by dissolving and redepositing chondrule material, just as it can do the same to sand grains in sandstone, and would best operate at lower temperatures (Rutter, 1976; Houseknecht, 1987; Skinner, 1989a, 1989b). It has also been argued that such close-fit textures occurred due to collision of chondrules prior to accretion, compaction-induced porosity loss, or even simple coincidence (Rubin, 1995b). Shock compaction may also be able to create close-fit textures (Davison et al., 2016), and is described in the shock metamorphism subsection of the background section (sec 2.6).

If it is true that cluster chondrites formed at high temperature then their existence establishes important constraints on chondrule formation. For chondrules to have accreted while hot would require them to be accreted onto or into planetesimals, or otherwise accrete into clumps, within hours of their formative heating, as chondrules cool rapidly after melting (e.g. Humayun, 2012; Miura and Yamamoto, 2014; Chaumard et al., 2018). This would imply that the proximity of an accreting planetesimal is likely correlated with chondrule heating, or that formation of planetesimals and chondrules occurs in the same 
process. This would be consistent with some bow shockwave (e.g. Morris et al., 2012; Mann et al., 2016) and impact splashing models (e.g. Dullemond et al., 2016; Lichtenberg et al., 2018). Further, the clast components of cluster chondrites would imply that shortly after heating events, the affected space of the nebula must contain a high proportion of chondrules to dust, which would favor models that heat entire local volumes, such as the shockwave models and the x-wind model (e.g. Shu et al., 2001; Morris et al., 2016). As a result, constraining the accretion temperatures of cluster chondrites has the potential to advance the debate over the mechanism of chondrule formation. Accordingly, determining the accretion conditions of cluster chondrites is the primary purpose of this study.

To accomplish this, a number of type 3 ordinary chondrites were shock classified to identify unshocked chondrites. Four of these are selected for electron backscatter diffraction (EBSD) analysis, chosen to represent the full range of cluster texture quality and for good preservation from terrestrial weathering. EBSD is used to determine accretion temperatures of cluster chondrite chondrules. This is because of its ability to constrain deformational temperature by analysis of olivine dislocation slip system activation (Ruzicka and Hugo, 2018). Individual chondrules are also analyzed to look for relations between temperature and deformation, and to test Metzler's (2012) inference of deformational temperature from chondrule deformation. Details concerning shock, EBSD, and chondrule analysis can be found in the Methods section. Background on chondrites and chondrules are provided in the following section to support later discussion on this study's implications for chondrule formation models. Further background information on 
the utility of olivine dislocation systems and EBSD for determining deformational temperature are also in the following section.

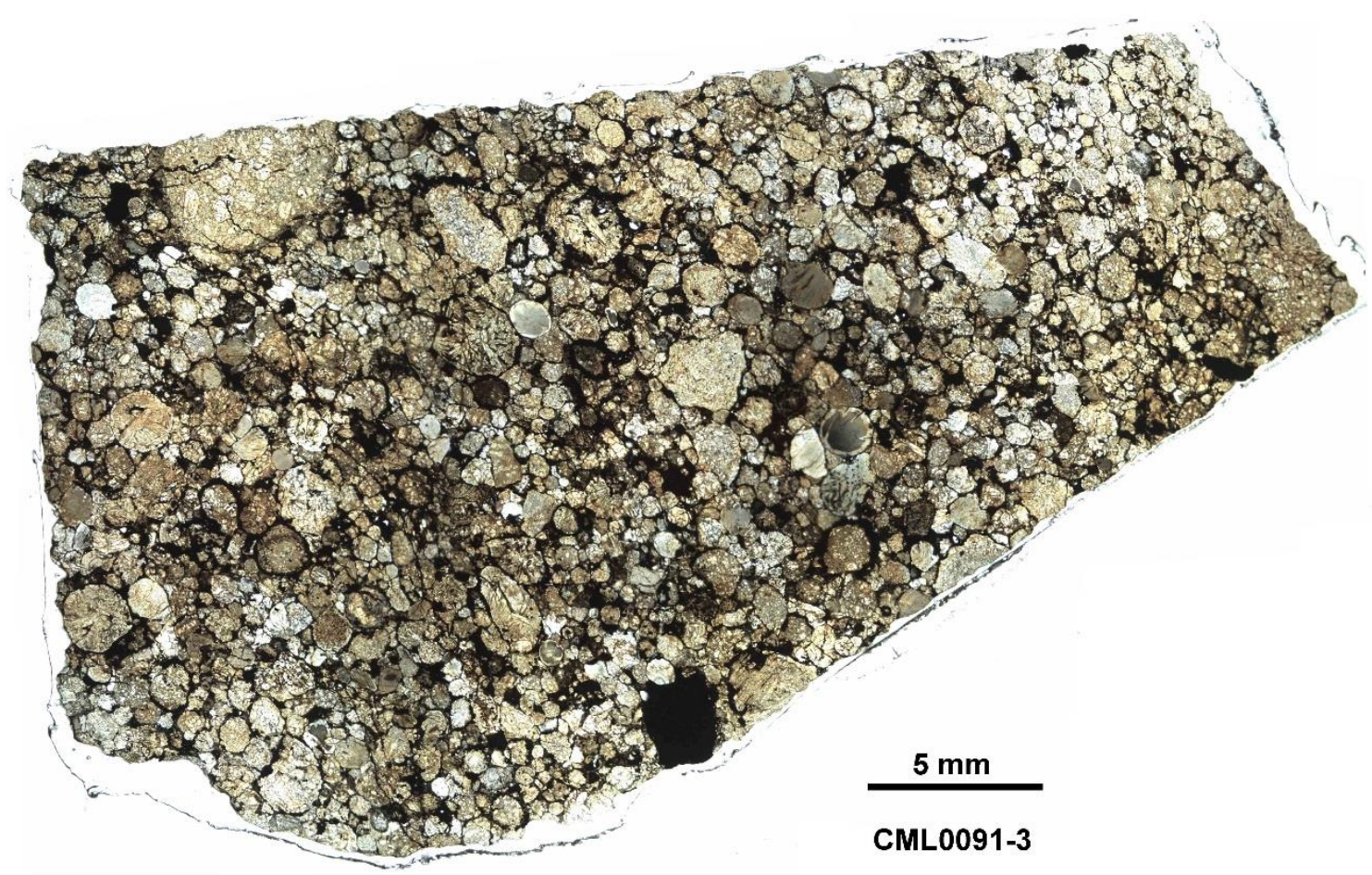

Figure 1. Example Image of a Cluster Chondrite. Sample is a section of NWA 11991. Possibly deformed chondrules in a close fit texture are visible. Image courtesy of Alex Ruzicka. 


\section{Background}

\subsection{Chondrites}

All cluster chondrites are a textural type of chondrite. Chondrites were first described more than two centuries ago and have been a subject of study since then (Howard, 1802). Chondrites are the most abundant of the two main types of meteorites that fall to Earth, constituting $\approx 87 \%$ of falls (Wood, 1988). They are distinguished from the other type of meteorite, achondrites, by their comparatively primitive nature. Whereas achondrites are mostly igneous rocks from differentiated bodies, chondrites have never undergone processes of melting and differentiation (Weisberg et al., 2006). Chondrites instead are primitive accretionary rocks; rocks that formed from the amalgamation of dust and clasts from the solar nebula (Weisberg et al., 2006). Chondrites and their components date from the formation of the solar system, having formed within its first $5 \mathrm{Ma}$ (Amelin et al., 2002; Connolly et al., 2006; Hutcheon et al., 2009; Jones et al., 2000; Bizzarro et al., 2017; Kita et al., 2005; Krot et al., 2009; Connelly et al., 2008; Kita and Ushikubo, 2012; Bollard et al., 2017; Becker et al., 2015; Rudraswami and Goswami, 2007; Connelly et al., 2012; Connelly and Bizzarro, 2009; Amelin and Krot, 2007; Villeneuve et al., 2009; Wadhwa et al., 2007; Yurimoto and Wasson, 2002; Yin et al., 2007; Kita et al., 2000; Budde et al., 2018; Scott, 2007). Chondrites have two principal components: matrix and clasts. The dominant clasts in chondrites are chondrules, though other clasts are present in much lower abundance such as calcium-aluminum inclusions and amoeboid olivine aggregates, both of which are known as refractory inclusions (Weisberg et al., 2006). Matrix in chondrites is postulated to have formed by a number of mechanisms, including 
condensation of solids in the solar nebula and fragmentation of chondrules and other objects (Alexander, et al., 1989).

Chondrites have varying degrees of thermal metamorphism at elevated temperature and low pressure (Huss et al., 2006). This is characterized by a 3 to 6 scale based on petrographic and mineral composition features created by Van Schmus and Wood (1967), with 3 being unequilibrated and 6 being the highest degree of metamorphism prior to melting; grade 3 is further subdivided (i.e. 3.0-3.9; Grossman and Brearley, 2005; Huss et al., 2006). Temperature ranges for end point petrographic grades are $250-600^{\circ} \mathrm{C}$ for type 3 and $850-950^{\circ} \mathrm{C}$ for type 6 (McSween et al., 1988; Huss et al., 2006). Types 3.0-3.5 ordinary chondrites experienced peak temperatures of $300-450^{\circ} \mathrm{C}$ (Brearley, 1990).

Chondrites are divided into a number of classes on the basis of their chemistry, mineralogy, and petrology. In this study only LL, L, H group chondrites are used, which together form the ordinary chondrite class. Ordinary chondrites, which constitute $\sim 80 \%$ of falls, are matrix poor (10-15 vol\% matrix) and have few refractory inclusions. They are reduced compared to terrestrial rocks, but are neither exceptionally reduced or oxidized compared to other meteorites (Weisberg et al., 2006).

\subsection{Chondrules}

Chondrules are the primary component comprising cluster chondrites. These objects are submillimeter ultramafic igneous spherules, or fragments thereof, commonly found in chondrites (Hewins, 1997; Zanda, 2004; Scott and Krot, 2005; Lauretta et al., 2006). While having a generally subspherical shape, many chondrules are elongated by gas streamlining in the solar nebula, rendered oblate by rotation while in a molten state, or 
fragmented after formation. However, a large portion of chondrules have irregular shapes not explainable by such processes (Charles et al., 2018). They are commonly composed of olivine and/or pyroxene crystals with a feldspathic glass mesostasis (Hewins, 1997; Lauretta et al., 2006).

Chondrules are dominantly porphyritic in texture, though supercooling-related cryptocrystalline, barred, and radial textures are not uncommon (Gooding and Keil, 1981; Hewins, 1997; Connolly et al., 1998; Lauretta et al., 2006). Nonporphyritic chondrules likely formed in spatial environments with a higher abundance of coarser solids than porphyritic ones, as has been inferred from their higher abundance among compound chondrules and cratered chondrules (Gooding and Keil, 1981). Rapid crystallization in such chondrules was likely the result of dust colliding with molten chondrules, thereby providing nucleation sites (Connolly and Hewins, 1995). In comparison, porphyritic chondrules formed from incompletely melted precursors or experienced much slower cooling times (Hewins and Radomsky, 1990; Radomsky and Hewins, 1990). Thermal history is not the sole determiner of chondrule textures; precursor composition also plays an important role, as ferroan chondrules will have lower liquidi than magnesian ones (Connolly and Hewins, 1991). Chondrules vary bimodally in their iron and magnesium content. Type I chondrules have an $\mathrm{Fe} /(\mathrm{Fe}+\mathrm{Mg})$ molar ratio of $<0.1$. Others are considered to be Type II chondrules (Hewins, 1997; Zanda, 2004; Scott and Krot, 2005; Lauretta et al., 2006). Accordingly, chondrules of differing texture types could have the same thermal history even if they differ in composition. 
Numerous studies of chondrules have established several constraints that any genetic model must be able to meet. As is indicated by several radiogenic chronometers, these objects formed in the solar nebula, with most forming 2-3 Ma after calciumaluminum inclusions (CAIs; $\left.\Delta \mathrm{t}_{\mathrm{CAI}}=2-3 \mathrm{Ma}\right)$, though some formed much earlier $\left(\Delta \mathrm{t}_{\mathrm{CA}} \approx 0\right.$ Ma) or later $\left(\Delta \mathrm{t}_{\mathrm{CA}} \approx 5 \mathrm{Ma}\right)$ (Jones et al., 2000; Kita et al., 2000; Amelin et al., 2002; Yurimoto and Wasson, 2002; Kita et al., 2005; Connolly et al., 2006; Amelin and Krot, 2007; Rudraswami and Goswami, 2007; Scott, 2007; Wadhwa et al., 2007; Yin et al., 2007; Connelly et al., 2008; Connelly and Bizzarro, 2009; Hutcheon et al., 2009; Krot et al., 2009; Villeneuve et al., 2009; Connelly et al., 2012; Kita and Ushikubo, 2012; Becker et al., 2015; Bizzarro et al., 2017; Bollard et al., 2017; Budde et al., 2018). Chondrules probably experienced abrupt heating to temperatures of $1600-2400 \mathrm{~K}$ from initial temperatures of $600 \mathrm{~K}$, and cooled at rates ranging from $0.5-8000 \mathrm{~K} / \mathrm{hr}$ in a dust-filled environment, based on their retention of $\mathrm{Na}$ and other moderately volatile elements, a lack of olivine reequilibration in 3.0-3.2 grade chondrites, and a lack of isotopic fractionation of Fe and $\mathrm{S}$ (Hewins, 1983; Lofgren and Russell, 1986; Hewins and Radomsky, 1990; Radomsky and Hewins, 1990; Hewins, 1991; Jones and Lofgren, 1993; Müller et al., 1995; Lofgren, 1996; Hewins, 1997; Connolly et al., 1998; Weinbruch et al., 1998; Cohen et al., 2000; Jones et al., 2000; Alexander and Wang, 2001; Yurimoto and Wasson, 2002; Yu et al., 2003; Tsuchiyama et al., 2004; Wasson, 2004; Zanda, 2004; Hewins et al., 2005; Tachibana and Huss, 2005; Connolly et al., 2006; Lauretta et al., 2006; Tachibana, 2006; Nagashima et al., 2008; Miyamoto et al., 2009; Alexander and Ebel, 2012; Desch et al., 2012; Humayun, 2012; Miura and Yamaoto, 2014; Villeneuve et al., 2015; Connolly and Jones, 2016; Soulié 
et al., 2017; Chaumard et al., 2018). On the basis of suppressed Raleigh fractionation, it has been argued that chondrules must have formed in spatially large regions of heating (Cuzzi and Alexander, 2006; Desch, 2006). The conditions of melting also require higher than canonical oxygen fugacities to retain $\mathrm{Na}$ and metallic iron in chondrule melts (Lewis et al., 1993; Hewins, 1997; Cohen and Hewins, 2004).

It has also been inferred from many lines of evidence that chondrules must have formed in an environment with a very high concentration of chondrules and other solids, as this would explain the incidence rate of compound chondrules, lack of isotopic fractionation from evaporation effects, and scarcity of glassy chondrules (Connolly and Hewins, 1995; Ciesla et al., 2004; Ciesla, 2006; Desch, 2006; Alexander and Ebel, 2012; Hewins et al., 2012; Arakawa and Nakamoto, 2016). The densities of chondrules implied by these observations are so high that such a cloud of chondrules would likely collapse under its own gravity (Alexander and Ebel, 2012). This possibly conflicts with another well-established constraint: that chondrules were oft subject to multiple heating events prior to accretion, as is evidenced by relict grains, crystal zoning, igneous rims, and textural distributions (Fox and Hewins, 2005; Kracher et al., 1984; Palme et al., 1992; Rubin and Krot, 1996; Hewins, 1997; Jones et al., 2000; Yurimoto and Wasson, 2002; Wasson and Rubin, 2003; Alexander, Barber, et al., 1989; Scott and Krot, 2005; Connolly et al., 2006; Lauretta et al., 2006; Ruzicka et al., 2007, 2008; Alexander and Ebel, 2012; Rubin, 2013; Baecker et al., 2017). Such a contradiction could be solved however, if such formed bodies were subsequently disrupted, allowing their components to be reheated in the nebula (Ruzicka, 2012). 
Additional constraints on chondrule origins are given by their compositions and sizes (Ciesla, 2005). For example, chemical complementarity between chondrules and matrix in chondrite groups, such that combined they match solar elemental abundance even if the chondrules and matrix by themselves do not, requires that chondrules and matrix are created or affected jointly during chondrule formation (Bland et al., 2005; Hezel and Palme, 2008, 2010; Desch et al., 2012; Palme et al., 2015; Budde et al., 2016). A final constraint is the low variance in chondrule sizes within chondrite groups, which favors models that can explain such limited size ranges (Jacquet, 2014).

\subsection{Chondrule Formation Models}

A number of models have been proposed since 1877 to explain the formation of chondrules, but the debate of which is correct has remained unsettled since that time (Sorby, 1877). The most currently favored formation models are the shockwave models (Connolly and Jones, 2016; Krot and Nagashima, 2017). Shockwave models posit that chondrules and their precursors were heated by supersonic shockwaves in the solar nebula (Connolly and Love, 1998; Jones et al., 2000; Desch et al., 2005; Connolly et al., 2006). The primary mechanism of heating is friction by gas drag once particles enter the shockwaves, though secondary heating effects such as thermal radiation from heated particles in a shockwave prior to entry and compressional heating of the nebular gas may also contribute (Connolly and Love, 1998; Desch and Connolly, 2002; Desch et al., 2005; Connolly et al., 2006). Gas drag in the shockwave would slow chondrules to the same speed as the shockwave, and the objects should be able to survive that deceleration (Connolly and Love, 1998; Sirono, 2006). Shockwaves are capable of producing a range 
of temperature environments, including those consistent with chondrule thermal histories, if the right conditions are met (Ciesla, 2005; Desch et al., 2005). A particular strength of many shockwave models is their ability to explain the high concentrations of both gas and solids inferred for chondrule formation, as the shockwaves would act to concentrate both and vaporize solids (Connolly and Love, 1998; Desch and Connolly, 2002; Boss and Durisen, 2005b). Another is the strong fit between the modeled thermal histories of particles moving through shockwaves and the thermal histories inferred for chondrules (Desch and Connolly, 2002; Desch et al., 2005; Connolly et al., 2006). Shockwaves can also explain the limited size ranges of chondrules, stripping melt from larger particles and vaporizing smaller ones (Miura and Nakamoto, 2005; Kato et al., 2006; Miura and Nakamoto, 2007). Further, shockwaves are easily recurring, and so can account for the abundant evidence for chondrule reheating (Ciesla, 2005; Desch et al., 2005). As shockwaves can affect large portions of the solar nebula, they can thermally process a large amount of solids (Ciesla, 2005). Finally, shockwaves are consistent with the cogenetic relationship of chondrules and matrix implied by complementarity (Palme et al., 2015).

A multitude of sources for shockwaves have been proposed: bow shocks around eccentric planetesimals, density waves in the solar nebula, $\mathrm{x}$-ray flares from the young sun, gas clumps falling into the solar nebula, tidal interactions with other stellar systems, and mass accretion into the solar nebula (Boss, 1996; Larson, 2002; Boss and Durisen, 2005b; Desch et al., 2005; Nakamoto et al., 2005; Connolly et al., 2006). X-ray flares would have occurred as a result of mass accretion to the young Sun, and could have generated substantial nebular shockwaves in the outer surface of the solar nebula (Nakamoto et al., 
2005). Shockwaves in the periphery of the solar disk could also be created by accretion of clumps of dust and gas, though like x-ray flares these would not affect the midplane of the solar nebula (Tanaka et al., 1998; Boss and Durisen, 2005b). A similar model works by the accretion of in-falling gas (Boss, 1996; Ruzmaikina and Ip, 1996; Boss and Durisen, 2005b). A further proposed source for shockwaves is tidal interactions with other proplyds in a densely packed stellar nursery (Larson, 2002). Issues with these particular models include their location away from the nebular midplane, where the solids are concentrated, and a difficulty in explaining how chondrules could be reheated (Boss and Durisen, 2005b). Further, these mechanisms are not expected to have operated in the timeframe that most chondrules were created (Boss and Durisen, 2005b).

The two most popular shockwave models are those of gravitational instabilities and bow shocks. In the former, shockwaves are caused by gravitational instability waves, that would be capable of processing material in the nebular midplane in a manner which has good agreement with inferred chondrule thermal histories (Hood and Horányi, 1991; Desch and Connolly, 2002; Miura et al., 2002; Ciesla and Hood, 2002; Boss and Durisen, 2005b; Desch et al., 2005; Hood et al., 2009; Morris and Desch, 2010; Morris et al., 2016). Numerical modeling studies indicate that an early formed Jupiter could generate such density shockwaves in the solar nebula (Boss and Durisen, 2005a). Additional modeling work has indicated that the required early formation of Jupiter is plausible, if not required (Boss, 2000; Hood et al., 2009; Mann et al., 2016). This modeling work has empirical support from work on iron meteorites that suggests the formation of two distinct 
geochemical reservoirs in the solar proplyd by $1 \mathrm{Ma}$, which is best explained by the formation of Jupiter (Kruijer et al., 2017).

The second popular model is that of chondrule heating in bow shocks, shockwaves around rapidly moving planetesimals and protoplanets (Hood, 1998; Weidenschilling et al., 1998; Ciesla, 2005; Desch et al., 2005; Hood et al., 2005, 2009; Hood and Weidenschilling, 2012; Morris et al., 2012). Early formed planetesimals could have been perturbed into eccentric orbits by proto-Jupiter, achieving high speeds relative to the nebular gas and thermally processing significantly large areas of the solar proplyd with their bow shocks before regaining a circular orbit (Hood, 1998; Weidenschilling et al., 1998; Marzari and Weidenschilling, 2002; Boss and Durisen, 2005b; Desch et al., 2005; Nagasawa et al., 2014). There is substantial evidence that planetesimals and planetary embryos had already formed before most chondrules did. Such evidence includes the dating of iron meteorites, angrites, and the core formation of Mars, observations of igneous clasts with chemistry consistent with differentiated parent bodies found in chondrites, and observations of granoblastic relict olivine textures in chondrules, though it has been argued that the latter of these does not require such an origin (Kennedy et al., 1992; Hutchison, 1996a; Lugmair and Shukolyukov, 2001; Baker et al., 2005; Kleine et al., 2005; Scherstén et al., 2006; Libourel and Krot, 2007; Sokol et al., 2007; Whattam et al., 2008; Dauphas and Pourmand, 2011; Libourel and Chaussidon, 2011). It is probable that such a planetesimal would need to be large. This would give chondrules time to be mechanically coupled to the larger bow shock. This coupling would avoid accretion and extend modelled chondrule cooling times to be consistent with chemical and petrologic observations (Hood 
et al., 2005; Mann et al., 2016). Chondrules that did not become coupled to the bow shock would be accreted to the planetesimal, and would not be thermally reprocessed and nor be likely to survive their collision with the planetesimal (Hood et al., 2005). Such larger planetesimals or planetary embryos could also have the advantage of possessing Na-rich outgassed atmospheres that could interact with forming chondrules, explaining their $\mathrm{Na}$ content (Morris et al., 2012). Further, modelling of chondrule thermal histories in bow shocks of planetary embryos is consistent with chondrule formation constraints (Morris et al., 2012). However, further modeling indicates that it may be the case that only larger objects can produce such thermal histories (Mann et al., 2016). It is also entirely feasible that both bow shock and density wave mechanisms could operate simultaneously, especially as both could be caused by an early-formed Jupiter (Boss and Durisen, 2005b).

Critics of shockwave models have used various arguments. Numerical modeling of the collisional disruption of chondrules encountering dust grains in the shockwave region by Jacquet and Thompson (2014) indicate that such collisions should destroy chondrules, but their model does not explain how dust could survive preheating so as to be in the shockwave region at all (Morris and Desch, 2010; Morris et al., 2016). Fedkin et al. (2012) used numerical modeling of kinetic chemical and isotopic fractionation under shockwave conditions to argue that the fractionations predicted by the modeling are inconsistent with geochemical data of chondrules. However, effects such as back-reaction are not accounted for by their model. It has also been argued on the basis of numerical modeling that the generation of nebular shockwaves sufficiently powerful to create chondrules is unlikely (Boley and Durisen, 2008). Further, the good fit of shockwave 
heating to inferred chondrule thermal histories has been questioned by Stammler and Dullemond (2014). Similarly, modeling of a nebular shockwave by Miura and Nakamoto (2006) found that thermal histories in their models did not agree with those inferred for chondrules, though more recent modelling studies do agree with chondrule thermal histories (Morris and Desch, 2010; Morris et al., 2016).

Currently discussed impact models generally resemble the model of Asphaug et al. (2011), which sees chondrules as resulting from impacts between young mostly-molten planetesimals (Lugmair and Shukolyukov, 2001; Sanders and Taylor, 2005; Sanders and Scott, 2012; Connolly and Jones, 2016). However, other contemporary solid or mostlysolid models exist (Johnson et al., 2015; Lichtenburg et al., 2017; Lichtenberg et al., 2018). Early versions of the model envisioned solid planetesimals colliding and spewing molten droplets, or the formation of chondrules as impact ejecta (Ruzicka et al., 2000; Hutchison et al., 2005). Such models are no longer favored, however, though recent versions have been supported with numerical modeling (Johnson et al., 2015). Instead, impact splashing models propose that chondrules result from oblique impacts of mostly molten early planetesimals. Heat provided by short-lived nuclides is thought to have melted these objects, and the models posit that they did not have time to differentiate so as to explain complementarity (Hevey and Sanders, 2006; Sanders and Scott, 2012). Collisions between these objects could have produced expanding clouds of droplets and vapor with cooling histories compatible with those inferred for chondrules (Sanders and Scott, 2012; Dullemond et al., 2014). Such collisions may have been numerous, and are estimated to have occurred about once per 240-24,000 years in the solar nebula (Hood et al., 2009). 
Chondrules generated by this model could also plausibly meet many other constraints, such as size ranges, ages of formation, and volatile contents (Sanders and Scott, 2012; Dullemond et al., 2016). Modeling of chondrule production by planetesimals indicates that this process could create a sufficient mass of chondrules in $10 \mathrm{Ma}$, too long for established chondrule and solar nebula chronology (Hasegawa et al., 2016). Chondrules in CR clan chondrites, especially those of the $\mathrm{CB}$ group but also some in the $\mathrm{CH}$ group, have many unusual features that are well explained from an impact-splashing origin (Krot et al., 2005, 2007; Bollard et al., 2015; Fedkin et al., 2015; Oulton et al., 2016; Krot and Nagashima, 2017; Hewins et al., 2018).

However, it is unlikely that all chondrules could have been formed by this process, as it is incompatible with Na-rich chondrules which are interpreted to have formed from CAI and AOA precursors and it cannot reheat chondrules (Boss, 1996; Ebert and Bischoff, 2016). An additional strike against this model is that Hf-W dating of lithophile-siderophile separation of materials in CR chondrites is cotemporal with chondrule formation in those rocks, which is inconsistent with those chondrules being sourced from a differentiated body (Budde et al., 2018). Further, chondrules and chondrites generally lack evidence of a differentiated source material and clasts of planetesimal origin are rare in chondrites (McSween, 1977; Boss, 1996). However, the versions of the impact splashing model that use undifferentiated bodies do not suffer this shortcoming (Lichtenberg et al., 2018). Additionally, impact models can be expected to produce a highly variant size population of chondrules inconsistent with what is observed in most chondrite groups (Jacquet, 2014). Further, complementarity is difficult to explain with impact models (Palme et al., 2015). 
Interestingly, the collisions of planetesimals may generate nebular shockwaves capable of forming chondrules, indicating that the collision and nebular shockwave mechanisms may be compatible (Hood et al., 2009).

Electromagnetic models for chondrule formation are of two primary types: conventional lightning models and current sheet models. Conventional lightning models are posited to have heated nebular solids both by Ohmic resistance heating and thermal radiation of heated material (Eisenhour et al., 1994; Boss, 1996). The primary proposed source of charging is the beta decay of long-lived isotopes such as ${ }^{40} \mathrm{~K}$. However, numerous studies have demonstrated that charge build ups in the solar nebula should dissipate rapidly and would overwhelm the electrical potential generated by this mechanism (Gibbard et al., 1997; Pilipp et al., 1998). This is problematic, because reproducing the cooling rates of chondrules requires lightning to occur frequently in a small volume of space (Fujii and Miyamoto, 1983). Another lightning model put forth by Desch and Cuzzi (2000) operates through triboelectric charging of turbulence-concentrated solids. Turbulent concentration is required for this model. Without it, even triboelectric charging in the solar nebula is quickly dissipated, except in situations involving low concentrations of dust in the solar nebula (Boss, 1996; Gibbard et al., 1997; Pilipp et al., 1998; Muranushi, 2010). This model can produce lightning energetic enough to melt solids but requires that short lived radioisotopes, such as ${ }^{26} \mathrm{Al}$, be heterogeneously distributed in the solar nebula or that chondrules be formed after such isotopes had decayed away, both of which conditions are inconsistent with empirical evidence (Amelin et al., 2002). A newer model proposes that ${ }^{26} \mathrm{Al}$ can be a charging source, so long as the environment is one of closely 
packed chondrules in a ring around a planetesimal or planetary embryo (Johansen and Okuzumi, 2018). However, regardless of the charge building model, lightning creates environments with conditions too oxidizing to be consistent with chondrule formation and is more likely to destroy dust aggregates than melt them (Poppe et al., 2010; Muranushi et al., 2012). Further, simulation of heating by lightning discharges indicates that heating rates are on the order of $10^{4} \mathrm{~K} / \mathrm{hr}$, too high to be consistent with the ranges of chondrule thermal history (Horányi and Robertson, 1996). The model of Johansen and Okuzumi (2018) is an exception, instead predicting cooling rates on the order of $10^{3} \mathrm{~K} / \mathrm{hr}$, which is a result of high particle densities not present in other lightning models.

The other primary model of electromagnetic heating is that of current sheets. In this model, heating is driven by magnetohydrodynamic interactions between the sun and the surface of the protoplanetary disk (Joung et al., 2004). The magnetic field of the protosun induces current sheets to form in the ionized surface of the disk (Joung et al., 2004). Short circuit instabilities could result in rapid flash-heating of solids in a current sheet (Joung et al., 2004; Hubbard et al., 2012, 2013). These instabilities would be triggered by rising temperatures, initiating at $1000 \mathrm{~K}$, far higher than the background preheating temperature inferred for chondrules (Hubbard et al., 2013). This effect could only act in the periphery of the disk, as its interior was a dead zone (Joung et al., 2004; Muranushi et al., 2012; Dzyurkevich et al., 2013). This dead zone would shrink over the lifetime of the solar nebula however, possibly allowing the current sheet to reach the midplane $\sim 2$ Ma after CAI formation (Joung et al., 2004). The current sheet model has been criticized by Desch and Turner (2015), whose numerical modeling predicts that the 
mechanism can only operate at temperatures that would have vaporized sulfur observed in chondrules from their precursors. Desch and Turner (2015) also argue that conditions capable of inducing current sheets to heat solids are incompatible with those conditions that allow current sheets to exist. However, current sheet models are capable of explaining complementarity (Palme et al., 2015).

The X-wind model proposes that chondrules and other igneous solids were created close to the young Sun at an "X-point," melted by its intense radiation and propelled outward into the disk by magnetically driven outflow (Shu et al., 1997, 2001). However, the X-wind model for chondrule formation has been heavily criticized. It is questionable that chondrules could retain their observed volatile contents while traveling outside the disk of the solar nebula (Ciesla, 2005). However, the unusual chondrules of the CR and $\mathrm{CH}$ chondrites lack such volatiles, and have been suggested to have formed by the $\mathrm{X}$-wind process (Krot et al., 2001). Further issues with the X-wind model have been raised by Desch et al. (2010), who argue it underestimates the degree of heating at the X-point, is self-contradictory on a theoretical basis, and cannot produce thermal histories consistent with those of chondrules. It is also argued that the X-wind model is unable to account for either complementarity or for the timing of chondrule formation (Palme et al., 2015).

It has been suggested that close-sourced gamma ray bursts could heat material in the solar nebula and form chondrules. Experiments by Duggan et al. (2003) have demonstrated that gamma rays can melt nebular solids and produce chondrule-like objects. It is also feasible that a gamma ray burst could induce widespread lightning in the solar nebula by ionizing atoms and propelling electrons in a coherent cloud far away from their 
source ions (McBreen et al., 2005; Winston et al., 2006). However, the probability of such a gamma ray burst occurring as often as once within close enough vicinity of the solar nebula over its lifetime has been calculated as being 0.1\% (McBreen and Hanlon, 1999). Accordingly, recurrent heating by this mechanism, as is required by differing formation dates in chondrules and pervasive evidence of reheating in chondrules, is difficult to explain, and other constraints, such as the highly concentrated environment of chondrule melts, remain unaddressed.

\subsection{Dislocations}

Dislocations in olivine grains are used in this study to infer the accretional temperatures of cluster chondrite chondrules. Dislocation creep is the primary mechanism of intracrystalline plastic deformation (Weertman and Weertman, 1992; Passchier and Trouw, 2005). It consists of the movement of linear crystal defects constrained to a plane through a mineral grain (Weertman and Weertman, 1992; Passchier and Trouw, 2005). There are two types of dislocations: edge dislocations and screw dislocations, see Figure 2 (Weertman and Weertman, 1992; Passchier and Trouw, 2005). Edge dislocations represent an extra half-plane in the crystal lattice, whereas screw dislocations represent a twisting of the crystal lattice (Weertman and Weertman, 1992; Passchier and Trouw, 2005).

The orientation and movement of dislocations through a crystal is described in crystallographic terms, principally Miller indices. Minerals can be classified into six crystal classes on the basis of their symmetries (Klein and Dutrow, 2007). Each of these crystal classes has a unique system of crystallographic poles, and the orientation of any crystallographically oriented feature can be described in terms of these poles, typically 
through a number indicating that a feature intersects the pole $(\geq 1)$ or not $(0)$ (Klein and Dutrow, 2007). These numbers are listed in series, with their ordering indicating which pole each number is referencing. Orthorhombic minerals such as olivine have three different mutually perpendicular crystallographic poles: a, b, and c (Klein and Dutrow, 2007). A plane described as (001) in an orthorhombic crystal intersects the c pole and not the a or b poles. Accordingly, such a plane must be perpendicular to the c pole and parallel to the $\mathrm{a}$ and $\mathrm{b}$ poles, given the geometry of the system. A line described as [001] parallels the $\mathrm{c}$ pole and not the $\mathrm{b}$ or a poles. However, given the geometric nature of the object, this has the result of the line paralleling the $\mathrm{c}$ pole and being perpendicular to the a and $\mathrm{b}$ poles, which is the opposite case from the plane before.

The crystallographically controlled movement of dislocations through a mineral is defined through a slip plane and slip direction, both of which are described with Miller indices (Weertman and Weertman, 1992). Slip planes are described in the form of $(h k l)$ or $\{h k l\}$. The former signifies a singular slip plane, whereas the latter signifies a set of planes that all intersect with the defined poles (Weertman and Weertman, 1992; Passchier and Trouw, 2005). Slip directions, also referred to as Burgers (b) vectors, are described in the manner of lines in the form of $[u v w]$ (Weertman and Weertman, 1992; Passchier and Trouw, 2005). A slip plane and a Burgers vector together constitute a slip system, a complete crystallographic description of a specific dislocation creep mechanism in a mineral, and are simply written as $[u v w](h k l)$ or $[u v w]\{h k l\}$ (Weertman and Weertman, 1992; Passchier and Trouw, 2005). 
Dislocations can pile up into dislocation walls and subgrain boundaries, which divide slightly misoriented domains of the crystal (Weertman and Weertman, 1992; Passchier and Trouw, 2005; Fossen, 2016). The development of subgrains is enhanced by annealing, as dislocations can migrate and aggregate during the process (Weertman and Weertman, 1992; Passchier and Trouw, 2005; Fossen, 2016). Misorientations between subgrains can be represented by a rotation axis in a crystallographic rotation axis (CRA) plot, in a crystal coordinate reference frame. CRAs represent an axis around which a misoriented section of crystal lattice could be realigned with a neighboring nonmisoriented section of crystal lattice. CRA rotation axes are perpendicular to the Burgers vectors (or slip directions) of the dislocations in the boundary between the described regions (See Figure 3, Lloyd et al. 1997). As a result, the Burgers vector of a dislocation system can be constrained to a plane perpendicular to the CRA rotation axis, though its precise direction is unknown. This plane should not be confused with the slip plane or planes of a dislocation system. If the preferred dislocation systems of a mineral are well established in the literature and sufficiently limited in number, the plane of possible Burgers vectors can be sufficient to infer the probable dislocation system, and thereby be used to give information on the conditions associated with deformation. A CRA stereonet for interpreting slip systems in the specific case of olivine can be seen in Figure 4. 

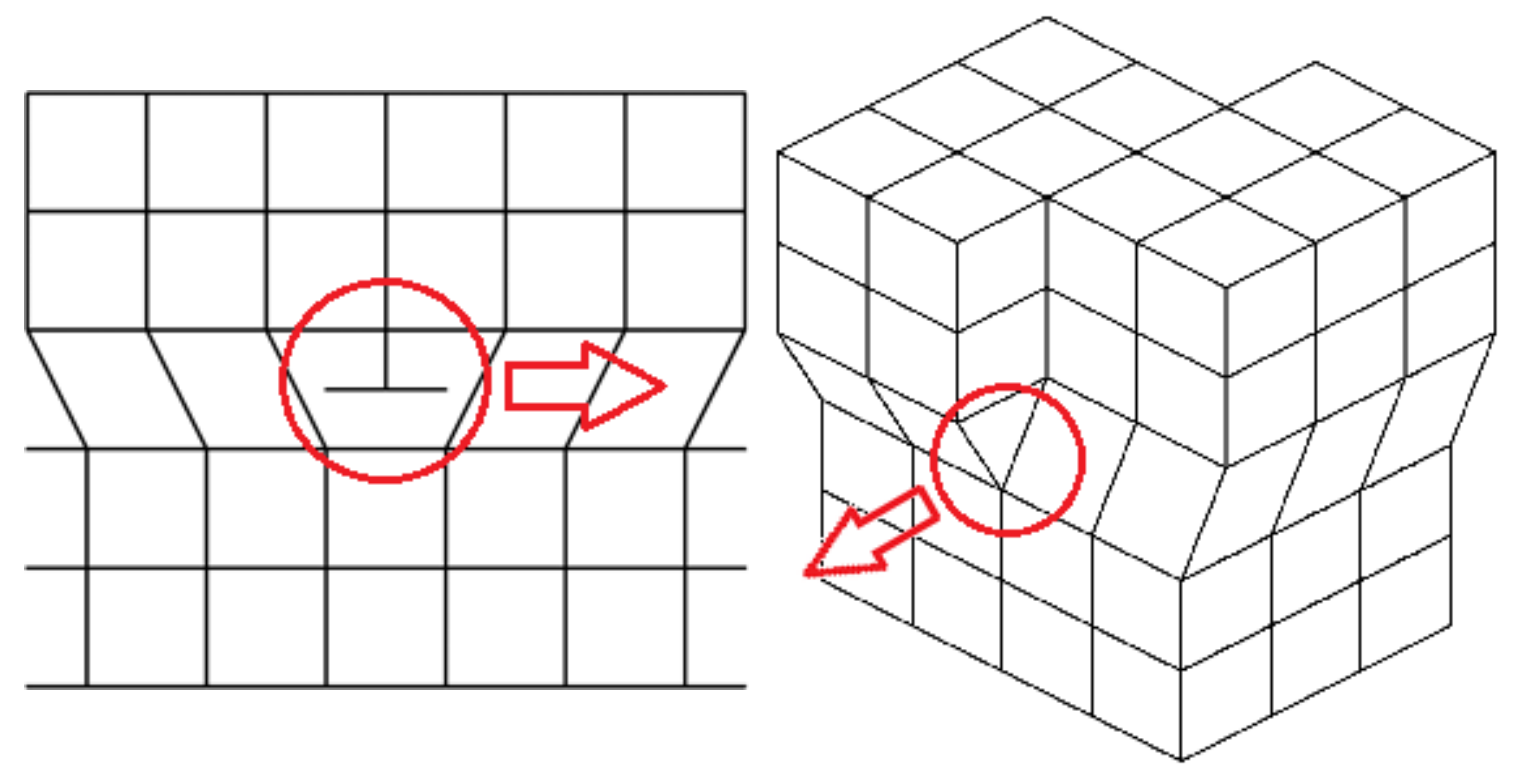

Figure 2. Cartoon Diagrams of Edge and Screw Dislocations. Left is an edge dislocation, right is a screw dislocation. Lines represent the crystal lattice; atoms are located in corners. After Fossen (2016). Dislocations are circled in red and Burgers vectors are indicated with red arrows.

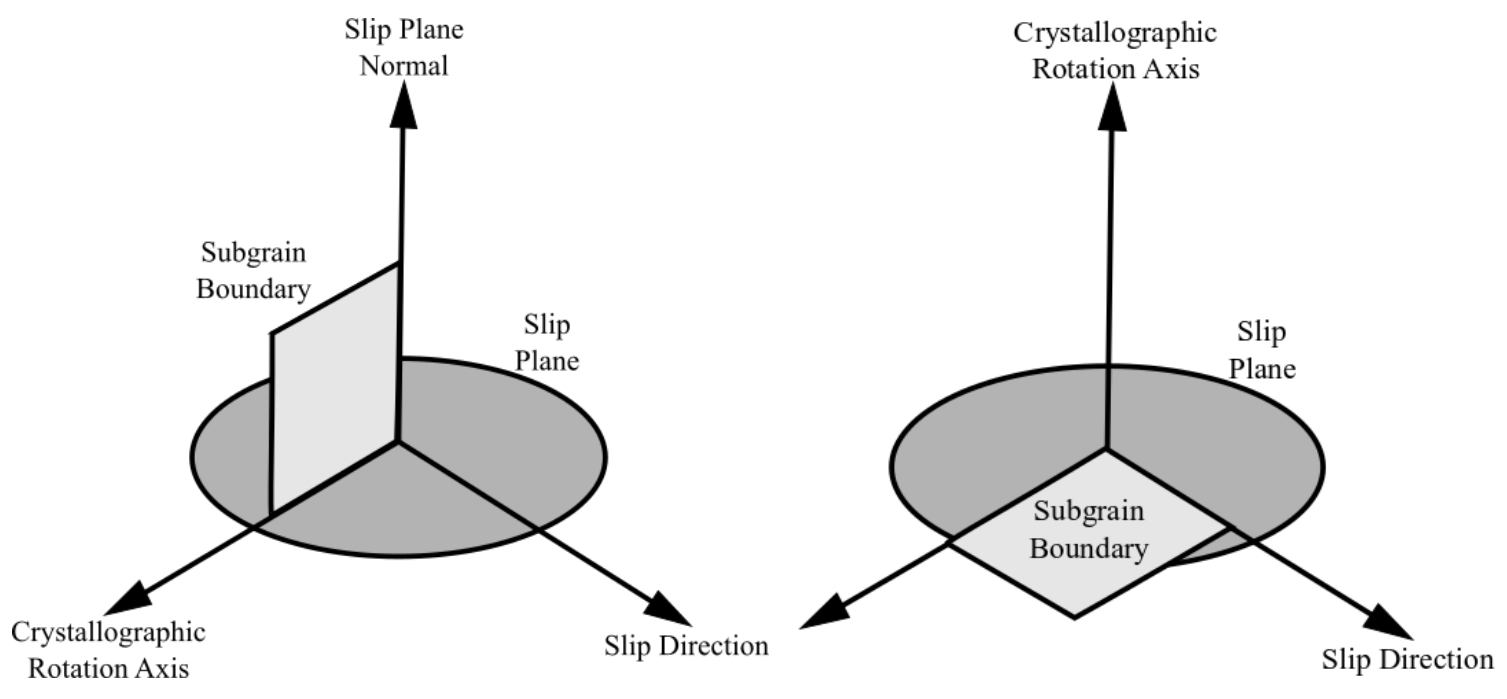

Figure 3. Geometric Relations Among Rotation Axes, Slip Systems, and Subgrain Boundaries. Slip direction is perpendicular to the crystallographic rotation axis. Right image is pertinent to screw dislocations, and left to edge dislocations. Adapted from Fig. 1b of Lloyd et al. (1997). 


\subsection{Olivine Deformation}

Olivine deformation is governed by a handful of primary dislocation slip systems that are active at different temperature ranges (Raleigh, 1968; Carter and Ave'Lallemant, 1970; Kirby and Wegner, 1978; Tommasi et al., 2000; Tielke et al., 2016). As olivine is common in cluster chondrite chondrules, identification of olivine slip systems is useful to infer codeformational temperature in these objects. The dislocation systems of olivine are (010)[100], $\{0 \mathrm{kl}\}[100]$, and $\{110\}[001]$ (Raleigh, 1965, 1967, 1968; Carter and Ave'Lallemant, 1970; Carter, 1971; Kirby and Wegner, 1978; Tommasi et al., 2000; Karato et al., 2008). They can be categorized by their Burgers vectors as either a-slip $(\mathbf{b}=[100])$ or c-slip $(\mathbf{b}=[001])$. While the activity of these systems is dependent on many factors including confining stress, water content, and oxygen fugacity, temperature is dominant over other factors (Raleigh, 1967, 1968; Carter and Ave'Lallemant, 1970; Tommasi et al., 2000; Karato et al., 2008). Although differing orientations of stress regimes can favor activation of one system over another, c-slip type systems are generally dominant at temperatures below $800^{\circ} \mathrm{C}$, a-slip type systems above $1000^{\circ} \mathrm{C}$, and a mixture of both system types occurs at $800-1000^{\circ} \mathrm{C}$ (Raleigh, 1967, 1968; Carter, 1971; Gaboriaud et al., 1981; Tommasi et al., 2000). Of the a-slip type systems, the (010)[100] system is dominant at higher temperatures than the $\{0 \mathrm{kl}\}[100]$ system (Carter and Ave'Lallemant, 1970; Carter, 1971; Karato et al., 2008). As for the other mechanisms, it is found that decreased pressure, high water content, and increased strain rates all favor c-slip (Carter and Ave'Lallemant, 1970; Durinck et al., 2005; Jung et al., 2006; Raterron et al., 2007, 2009; Tielke et al., 2017). 
Although much of the research on olivine deformation is oriented toward mantle conditions, extending the conventional temperature range of the c-slip to a-slip dominance transition to meteorites is not unreasonable even under the most extreme conditions. High water contents are unlikely for ordinary chondrites, and strain rate and pressure conditions can be expected to behave in a counteractive manner. Calculations based on the empirical relationships between confining pressure, strain rate, temperature for preferential slip system activation, and conditions appropriate for high impact shock conditions (10 GPa, $10^{-1}-10^{8} \mathrm{~s}^{-1}$ ) imply a transition temperature from c-slip to a-slip of $\approx 950^{\circ} \mathrm{C}$ (Raleigh, 1968 ; Carter and Ave'Lallemant, 1970; Carter, 1971; Stöffler et al., 1991; Schultz, 1996; Sharp and de Carli, 2006). Caution must be exercised with such a calculation, however, as it requires extending empirical mathematical relations far outside the range of strain rates and pressures in which they were derived. While the established effects on the transition temperature suggest reduction of it under the higher pressure conditions of shock metamorphism, observation of c-slip in cold shocked chondrites and a-slip in chondrites shocked while hot from thermal metamorphism suggest the transition remains in a reasonable temperature range (Carter et al., 1968; Ashworth et al., 1977; Müller and Wlotzka, 1982; Sneyd et al., 1988; Nakamura et al., 1992; Langenhorst et al., 1995; Leroux et al., 1996; Joreau et al., 1997; Leroux, 2001; Ruzicka and Hugo, 2018; Hugo et al., 2019). However, if the deformation observed in a sample does result from accretion processes rather than hypervelocity shock, the conditions of deformation should be much closer to those studied in the existing literature, rendering the matter less challenging. 


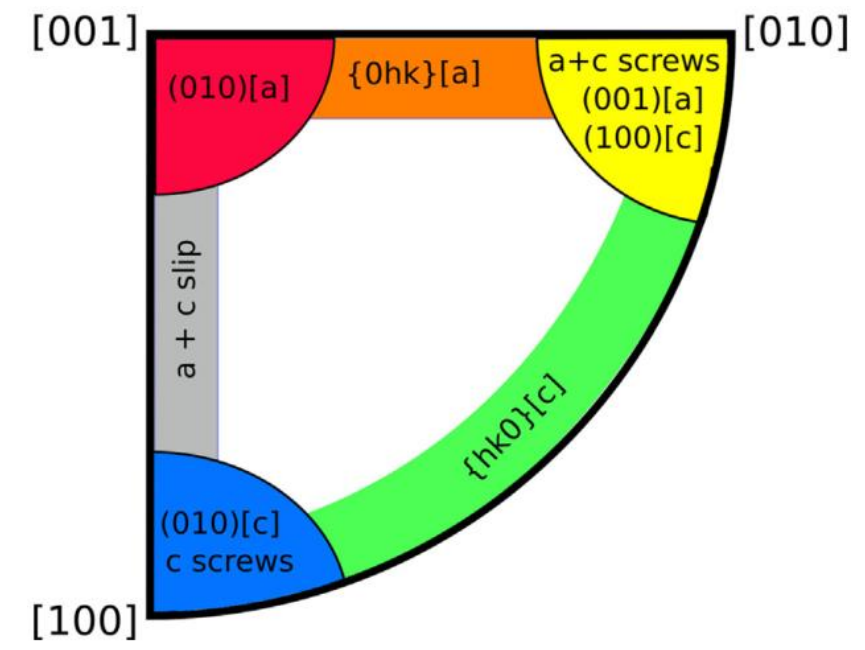

Figure 4. Diagram for Relating Crystallographic Rotation Axis Data to Olivine Slip Systems. Figure shows domains into which EBSD derived CRA data can be plotted, and which slip systems are associated with those domains. Diagram is a standard CRA-plot format and is an equal area stereonet section. Colors of domains are coded to interpreted temperature of deformation, from red (hot) to blue (cold). Figure is from (Ruzicka and Hugo, 2018), which itself is based in part on figure 2 of de Kloe et al. (2002).

\subsection{Electron Backscatter Diffraction}

EBSD is an analytical technique that can be used to map crystallographic orientations of mineral grains and subgrains in a sample. It employs use of a scanning electron microscope with a thin section sample inclined $70^{\circ}$ to the electron beam (Figure 5; Prior et al., 1999; Zaefferer, 2011). Electrons from the scanning beam penetrate the sample and diffract out through interactions with the crystal lattice of a mineral. The crystal lattice scatters diffracted electrons in an orderly manner that is dependent on its structure, which creates a crystallographically oriented diffraction pattern recordable by a detector (Prior et al., 1999; Zaefferer, 2011). As a result, a computer can rapidly determine the 
orientation of a mineral at a measured point from the diffraction patterns. A grid of such points can be measured across the sample, rastering an orientation map (Prior et al., 1999; Zaefferer, 2011).

Mapped EBSD data can be used to assess the overall intracrystalline deformation of olivine grains, as well as the slip systems active during olivine crystal deformation and the temperature conditions of that deformation. From internal orientation maps of single olivine crystals, misorientation boundaries can be identified and their CRAs compiled. Observed CRAs can be used to infer the slip systems active during deformation (de Kloe et al., 2002). Such analysis to determine slip systems of deformation has been successfully applied to olivine crystals by de Kloe et al. (2002) in experimentally deformed olivinepyroxene rocks and in meteorite samples by Ruzicka and Hugo $(2017,2018)$ and Hugo et al. (2019), with verification provided by transmission electron microscopy (TEM) analyses (Ruzicka et al., 2015; Hugo et al., 2019). CRA data can be used to infer the temperature of deformation, as the preferential activation of slip systems in olivine is temperature dependent (Ruzicka and Hugo, 2017, 2018). Mapped EBSD data can also be used to measure the grain orientation spread (GOS) and maximum grain orientation spread (MOS) values of crystals, which in meteoritic olivines are correlated to weighted shock stages determined by optical microscopy (Jamsja and Ruzicka, 2010; Ruzicka and Hugo, 2017, 2018; Hugo et al., 2019). 


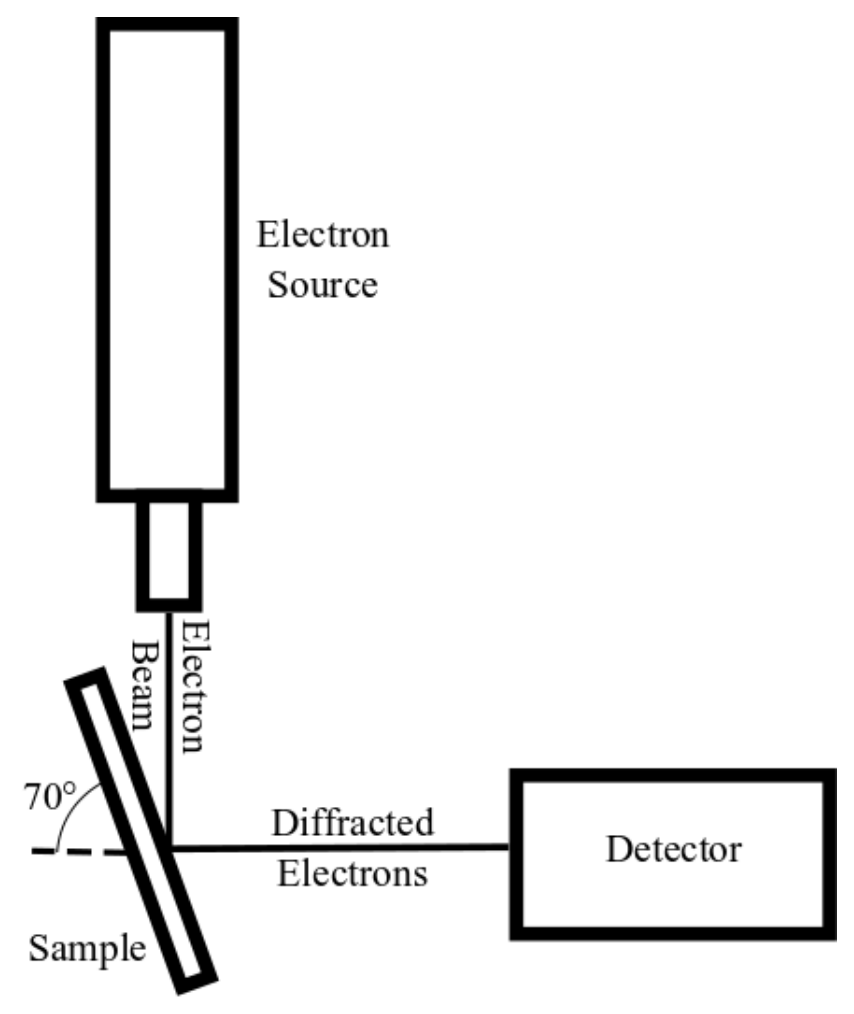

Figure 5. Set Up for EBSD Analysis with SEM. Figure is a cartoon diagram showing the basic configuration for EBSD analysis of a sample.

\subsection{Shock Metamorphism and Postshock Annealing}

Shock from impacts can deform olivine grains in chondrites, both brittlely and plasticly (Stöffler et al., 1988). Shock metamorphism is measured by an S1-S6 shock scale, with higher numbers signifying greater degrees of shock. The shock scale comes in two forms, the first being an integer system based on petrographic features of olivine and plagioclase created by Stöffler et al. (1991, 2018) and refined by Schmitt et al. (1994), Schmitt (2000), and Fritz et al. (2017). A second non-integer weighted shock stage scale was created by Jamsja and Ruzicka (2010). This latter scheme assigns a shock stage to each olivine grain based on shock-caused undulose extinction, planar fracturing, or recrystallization texture in olivine grains, associating data for individual crystals with 
conventional shock stages and then averaging all of the individual grain data to obtain a weighted shock stage for a sample.

While the degree of shock deformation is based on optically observable features, it has been established that these features correlate to the degree of olivine intracrystalline plastic deformation, including deformation resulting from dislocations (Carter et al., 1968; Nakamura et al., 1992; Ruzicka et al., 2015; Ruzicka and Hugo, 2017, 2018). Ruzicka and Hugo (2018) used EBSD derived mean grain orientation spread (GOS) metrics of olivine grains to characterize shock deformation, finding a good correlation $\left(r^{2}=0.94\right)$ between mean GOS and weighted shock stages in meteorites. Although mean GOS is still correlated to weighted shock stage in annealed meteorites, anomalously high GOS grains exist, indicating that skewness in GOS distributions can be evidence of annealed crystals.

Foliations with minor lineation components are commonly observed fabrics in meteorites, with impacts being commonly, though not always, cited as the most probable cause (Dodd, 1965; Cain et al., 1986; Scott et al., 1992; Nakamura et al., 1995; Tomeoka et al., 1999; Watt et al., 2006; Rubin and Swindle, 2011; Tait et al., 2013; Hanna et al., 2015; Forman et al., 2016, 2017). This process is called shock compaction, and it results in bimodal heating and deformation of chondrites, with matrix being much more affected than chondrules (Tomeoka et al., 1999; Bland et al., 2014; Davison et al., 2016). Numerical modeling indicates such compaction could deform chondrules in low-matrix chondrites, creating chondrule deformation and close-fit textures similar to those found in cluster chondrites (Davison et al., 2016). 
Thermal metamorphism can remove shock damage in minerals post-impact, restoring them to a low petrographic shock grade that contradicts their shock history (Ashworth and Mallinson, 1985; Rubin, 1992, 1995a; Ruzicka et al., 2015; Friedrich et al., 2017; Ruzicka and Hugo, 2017, 2018). Many meteorites show evidence for annealing after shock deformation (Ashworth et al., 1977; Leroux et al., 1996; Rubin, 2002, 2003; Rubin and Jones, 2003; Rubin, 2004; Rubin and Swindle, 2011; Friedrich et al., 2013, 2017). Annealing in meteorites can remove evidence for shock metamorphism from a meteorite (Ashworth and Mallinson, 1985; Friedrich et al., 2013, 2014, 2017). However, such annealed minerals still have microstructural evidence of shock in the form of dislocation configurations detectable by TEM (Ashworth and Mallinson, 1985; Ruzicka et al., 2015; Hugo et al., 2019). Annealing can also be inferred with EBSD data as GOS distributions are different for annealed and unannealed meteorites, with annealed meteorite GOS distributions being measurably skewed (Ruzicka and Hugo, 2017, 2018). 


\section{Methods and Samples}

\subsection{General Methodological Design}

The approach of this study followed three main methodological phases. 1) A number of type 3 OCs were shock classified with the aim of identifying unshocked samples. 2) Select unshocked samples were mapped twice with EBSD, and whole map data was analyzed so as to determine representative whole-sample deformation data and overall deformation temperature. 3) From the mapped data, 10 chondrules per map were subsetted and analyzed to determine their deformation character and deformation temperature. Then trends between deformation and temperature metrics were assessed for possible relations using ANOVA tests.

\subsection{Shock Classification}

To ensure the deformation measured with EBSD is not a result of shock and the observed cluster textures are not a result of shock compaction, the shock stages of all samples were measured so as to identify unshocked samples. This is not to say that cluster textures cannot be created by hypervelocity accretion and thereby have high shock stages. No conclusions along such lines are made in this study, but rather by using S1 samples deformational signals of post-accretion shock are minimized. This ensures the deformational signal observed is likely due to accretion.

Shock metamorphism was determined by assessment of shock features within the olivine grains of the samples by use of a Leica DM2500 petrographic microscope. Methods follows the guidelines of Stöffler et al. $(1991,2018)$ for determination of conventional shock stage and the methods of Jamsja and Ruzicka (2010) for determination of weighted 
shock stage. Shock stage character of olivine grains is determined as per the methods of Jamsja and Ruzicka (2010); the population of grains with a specific shock stage that composes a majority of observed grains ( $\geq 50 \%)$ is used to assign the conventional shock stage, or $\geq 25 \%$ of observed grains if no subpopulation constituted a majority of those observed. The former guideline is concordant with the procedure of Stöffler et al. (2018), and the latter with that of Stöffler et al. (1991). A summary of the criteria used by Jamsja and Ruzicka (2010) to classify the shock stage characters of olivine grains can be found in Table 1. For each grain this was done by measuring its angle of undulose extinction for the grain, counting the number of planar fractures sets in the grain, and noting whether the grain had been recrystallized or not. Weighted shock stage is determined as the mean of all assigned olivine shock stage characters, displayed with the standard deviation of the population (Jamsja and Ruzicka, 2010). Unlike as is recommended by Stöffler et al. (1991, 2018), plagioclase/maskelynite grains are not used for shock stage evaluation, as usable individuals of such grains are absent or rare in the samples due to their unequilibrated nature. Olivine grains used for analysis do not intersect the borders of the thin section and have minimal alteration and weathering, with the key criterion being that they are of sufficient recommended size ( $\geq 50 \mu \mathrm{m}$ in one dimension) and preservation to be able to display undulose extinction, planar fracture sets, and recrystallization should they have these features (Stöffler et al. 1991, 2018). Thin section samples were divided into sectors of approximately $6 \mathrm{~mm}^{2}$, and olivine shock character data was gathered for each for the purpose of mapping heterogeneity of shock character. Whole sample olivine shock data 
was used to determine both the conventional shock stage of the sample and the weighted shock stage of the sample.

Table 1. Summary of Jamsja and Ruzicka (2010) Classification Characteristics. Angle of undulose extinction is predominant over the number of planar fracture sets for purposes of shock stage character assignment.

\begin{tabular}{cl}
\hline Shock Stage & Criteria for Olivine \\
\hline S1 & Undulose Extinction Angle $<2^{\circ}$, No Planar Fracture Sets \\
S2 & Undulose Extinction Angle $2^{\circ}-3^{\circ}$, No Planar Fracture Sets \\
S3 & Undulose Extinction Angle $2^{\circ}-3^{\circ}, 1$ or More Planar Fracture Sets \\
S4 & Undulose Extinction Angle $3^{\circ}-5^{\circ}, 2$ or More Planar Fracture Sets \\
S5 & Undulose Extinction Angle $>5^{\circ}, 3$ or More Planar Fracture Sets \\
S6 & Recrystallization \\
\hline
\end{tabular}

\subsection{EBSD Methods}

Samples selected for EBSD analysis were chosen to fulfill three criteria: 1) low shock stage, as determined with the preceding shock methods, 2) low weathering, so as to use well preserved samples, 3 ) and all samples must collectively represent a wide range of cluster texture quality, so as to be representative of cluster chondrites as a whole. Once four samples were selected, they were analyzed with EBSD methods.

EBSD methods of Ruzicka and Hugo (2018) and Hugo et al. (2019) were used to gain CRA metrics for olivine subgrain boundaries in the samples and GOS metrics for olivine grains. A critical misorientation of $15^{\circ}$ was used to define grain boundaries in neighboring pixels of the same phase. CRA data were analyzed to determine causal slip systems for deformation in the olivine grains, and thereby infer temperature at deformation. GOS metrics were used to assess for evidence of annealed shock deformation in low shock grade samples by comparing mean GOS to median GOS, and to compare olivine deformation intensity to the shape parameters of the host chondrules. 
An accelerating voltage of $20 \mathrm{kV}$ was used for the EBSD work. EBSD mapping step sizes used ranged from 2 to $4 \mu \mathrm{m}$, with larger step sizes used for larger maps, so that all maps could be acquired in 24-48 hours. Minimum grain size was five contiguous pixels. Annealing parameters were computed by dividing the mean GOS by the median GOS of olivine grains larger in area than a $50 \mu \mathrm{m}$ diameter circle. Temperature parameters are calculated as the sum of the modal CRA densities, otherwise known as multiples of uniform density (MUD) values near the $<001>$ and $<010>$ poles relative to sum of the MUD values at all three poles, as these densities represent the activation of the a-slip systems favored in higher temperature regimes. Only misorientations of $2-10^{\circ}$ were used for constructing CRA diagrams. In CRA diagrams contouring was done based on a $25^{\circ}$ half-width.

\subsection{Chondrule Methods}

To determine the heterogeneity or homogeneity of chondrule accretion temperatures and to the test for other relations between GOS, shape parameters, and temperature, 10 chondrules from each map were selected and analyzed to determine their mean GOS, shape parameters, and temperature parameters. Temperature parameters for all samples were analyzed to determine their heterogeneity, and correlations between mean GOS, shape parameters, and temperature parameters were all tested for statistical significance. The purpose of this was test Metzler's (2012) inference of hot accretion from chondrule deformation, and whether chondrule and olivine deformation are linked.

Ten chondrules per EBSD map were chosen from each of the two maps of the four EBSD analyzed samples. The chosen chondrules were selected so as to represent the full spread of deformational variability of chondrules in the map. Deformational variability 
was determined by qualitative circularity, so that chondrules selected ranged from the most circular in shape within in a map to the least circular. Underlying this selection method is an assumption that undeformed chondrules are subcircular in cross-section. Chondrules with indistinct boundaries were avoided. Chondrules were chosen without regard as to their being chondrule fragments or not. Temperature parameters were determined for all chondrules, and mean GOS values and shape parameters were only determined for those selected chondrules that did not intersect the edges of a map, as it would be impossible to determine shape parameters for them. Temperature parameters were determined for chondrules as sums of modal densities near the poles of these subsets' CRA diagrams that are associated with olivine a-slip systems, as applied to chondrules subsetted from the EBSD maps using Channel 5 software. Shape parameters for chondrules were determined as the ratio of the perimeters of the chondrules and perimeters of circle with areas equal to those of the chondrules. This approach was intended to be consistent with "degree of chondrule deformation" used by Metzler (2012), which was interpreted by Metzler to reflect chondrule deformation on the assumption that undeformed chondrules were subspherical. ImageJ was used to measure chondrule perimeters and areas from band contrast images of the EBSD maps for the purposes of calculating the shape parameters. Mean GOS values for each chondrule were determined from the grain data of the chondrule subsets, with mean GOS being calculated as the average GOS of all olivine grains of a chondrule subset

Relations between the mean GOS, temperature parameters, and the shape parameters of the chondrules were analyzed sample by sample. Each metric was compared 
to another in pairs: mean GOS vs temperature parameters, mean GOS vs shape parameters, and shape parameters vs temperature parameters. Best fit lines were calculated for each of these metric pairs for each sample. These linear models were then analyzed using ANOVA tests, with a p-value of $5 \%$ being the threshold of statistical significance. If two or more samples show a significant correlation between a given pair of metrics, it is then determined that a consistent relationship exists. This is because when there is a $5 \%$ probability for each positive result to be a false positive, as would be case if the null hypothesis of no relation were true, then the probability of there being more than one false positive is $1.4 \%$, as calculated using a binomial distribution. This satisfies a 5\% p-value criterion.

Spatial variation in the CRA modality diagrams was calculated from automated subsets of EBSD whole maps, created by splitting the whole maps into 5 horizontal or vertical strips. The chosen orientation of the strips was selected to maximize their width. This created 10 regional subsets for each sample. Slip system modality for these strips was determined from their CRA diagrams, and 1.96 standard deviation ranges were calculated for each of the three slip systems for each sample. These formed the bounds of regional variation within the whole maps to which chondrule modal slip system activations could be compared.

\subsection{Study Samples}

Meteorites used in this study (Table 2, next page) are: Lut 006 (LL3), Moorabie (L3.8), NWA 1955 (H/L3-4), NWA 5205 (LL3.2), NWA 5421 (LL3.7), NWA 5781 (LL3.3), NWA 8575 (L/LL3), NWA 10220 (LL3), NWA 11291 (LL3), NWA 11351 (LL36), NWA 11905 (LL3.10-5), NWA 11991 (LL3), NWA 11992 (LL3), and Tieschitz (H/L 
3.6). All of these meteorites possess cluster chondrite lithologies and are type 3, characteristics which are necessary for this study. The low metamorphic type is needed so as to prevent the annealing of accretion-related dislocations by subsequent thermal metamorphism.

Some of the meteorites used in this study have existing research histories. NWA 5421 and NWA 5205 were previously examined by Metzler (2012) and Metzler and Pack (2016) to determine their chemistry and degree of deformation of their chondrules in their cluster chondrite clasts. Tieschitz is a much studied meteorite with evidence of aqueous alteration (Hutchison et al., 1998). It also has evidence of hot accretion in the form of indented chondrules and mesostasis expelled from chondrules during accretion (Hutchison et al., 1979, 1998). Lut 006 has been previously described by Hutson et al. (2014). These workers identified a texture of poorly foliated and closely packed chondrules in the meteorite, with a compositionally unusual matrix for its class.

Table 2. Table of Samples.

\begin{tabular}{lll}
\hline Name & Classification & Prior Shock Classifications \\
\hline NWA 5205 & LL3.2 (Weisberg et al., 2010) & None \\
NWA 5781 & LL3.3 (Weisberg et al., 2010) & None \\
NWA 5421 & LL3.7 (Weisberg et al., 2010) & None \\
Lut 006 & LL3 (Ruzicka et al., 2014) & S5 (Ruzicka et al., 2014) \\
NWA 10220 & LL3 (Bouvier et al., 2017) & S1 (Bouvier et al., 2017) \\
NWA 11291 & LL3 (Gattacceca et al., 2019) & None \\
NWA 11991 & LL3 (Hutson, Pers. Comm.) & None \\
NWA 11992 & LL3 (Hutson, Pers. Comm.) & None \\
NWA 11905 & LL3.10-5 (Agee, Pers. Comm.) & S3 (Agee, Pers. Comm.) \\
NWA 11351 & LL3-6 (Gattacceca et al., 2019) & S4 (Gattacceca et al., 2019) \\
NWA 8575 & L/LL3 (Ruzicka et al., 2014) & S1 (Ruzicka et al., 2014) \\
Moorabie & L3.8 (Clarke, 1975) & S4 (McCoy et al., 1994) \\
Tieschitz & H/L3.6 (Grady, 2000) & S1/2 (Scott et al., 1992), S3 \\
& & (Rubin et al., 1999) \\
NWA 1955 & H/L3-4 (Russell et al., 2005) & None \\
\hline
\end{tabular}




\section{Results}

\subsection{Shock Classification Results}

A number of samples were observed to be S1 (Table 3), four of which, 1) 0244-2

NWA 5781, 2) 0953-3A NWA 5421, 3) 0954-1A-1 NWA 5205, and 4) 0781B Tieschitz were chosen for EBSD analysis. Figures 6-9 display olivine shock stage grain histograms for each EBSD analyzed sample. Figures 10-13 display the spatial distribution of the shock data in these samples. Shock histogram figures and shock spatial distribution figures for samples not selected for EBSD analysis can be found in the Appendix. Shock data for the all samples are shown in Table 3.

Table 3. Olivine Shock Stage Data for Studied Samples. Columns 2 through 7 list number of observed grains with features consistent with the listed shock stage in a sample. A shock stage of "NA" means no shock stage can be assigned to a sample. Shock stages with "**" meet the criteria of Stöffler et al. (1991) but not of Stöffler et al. (2018). The " $n$ " column lists the number of grains measured in a sample.

\begin{tabular}{lccccccccc}
\hline \multicolumn{1}{c}{ Sample } & S1 & S2 & S3 & S4 & S5 & S6 & $\begin{array}{c}\text { Weighted } \\
\text { Shock Stage }\end{array}$ & $\begin{array}{c}\text { Shock } \\
\text { Stage }\end{array}$ & n \\
\hline 0091-3 NWA 11991 & 9 & 4 & 5 & 27 & 441 & 21 & $4.87 \pm 0.69$ & S5 & 507 \\
0244-2 NWA 5781 & 253 & 4 & 8 & 37 & 54 & 1 & $1.99 \pm 1.60$ & S1 & 357 \\
0545-2A Moorabie & 1 & 2 & 2 & 15 & 121 & 7 & $4.85 \pm 0.64$ & S5 & 148 \\
0677-2 NWA 11992 & 79 & 13 & 12 & 79 & 45 & 0 & $2.99 \pm 1.61$ & NA & 228 \\
0736-3 Lut 006 & 0 & 0 & 2 & 20 & 165 & 1 & $4.88 \pm 0.37$ & S5 & 188 \\
0754-2 NWA 11351 & 92 & 1 & 8 & 60 & 161 & 2 & $3.63 \pm 1.73$ & S5* & 324 \\
0754-3 NWA 11351 & 1 & 0 & 1 & 13 & 74 & 2 & $4.81 \pm 0.59$ & S5 & 91 \\
0781A Tieschitz & 304 & 9 & 0 & 11 & 8 & 0 & $1.22 \pm 0.81$ & S1 & 332 \\
0781B Tieschitz & 459 & 9 & 2 & 11 & 15 & 0 & $1.21 \pm 0.82$ & S1 & 496 \\
0856-2A NWA 1955 & 11 & 19 & 43 & 48 & 4 & 0 & $3.12 \pm 1.00$ & S4* & 125 \\
0953-3A NWA 5421 & 351 & 3 & 7 & 14 & 16 & 0 & $1.31 \pm 0.98$ & S1 & 391 \\
0953-3B NWA 5421 & 262 & 9 & 13 & 19 & 12 & 0 & $1.44 \pm 1.07$ & S1 & 315 \\
0954-1A-1 NWA 5205 & 1022 & 20 & 63 & 132 & 66 & 1 & $1.62 \pm 1.25$ & S1 & 1304 \\
0978-1A NWA 11905 & 268 & 0 & 6 & 10 & 20 & 0 & $1.40 \pm 1.13$ & S1 & 304 \\
0978-2A NWA 11905 & 77 & 0 & 1 & 1 & 7 & 0 & $1.38 \pm 1.15$ & S1 & 86 \\
0983-A NWA 11291 & 336 & 13 & 2 & 12 & 1 & 0 & $1.16 \pm 0.61$ & S1 & 364 \\
0983-B NWA 11291 & 333 & 12 & 3 & 7 & 4 & 0 & $1.15 \pm 0.63$ & S1 & 359 \\
0984-A NWA 10220 & 103 & 5 & 1 & 5 & 1 & 0 & $1.23 \pm 0.75$ & S1 & 115 \\
0985-A NWA 8575 & 233 & 8 & 0 & 6 & 1 & 0 & $1.12 \pm 0.55$ & S1 & 248 \\
\hline
\end{tabular}




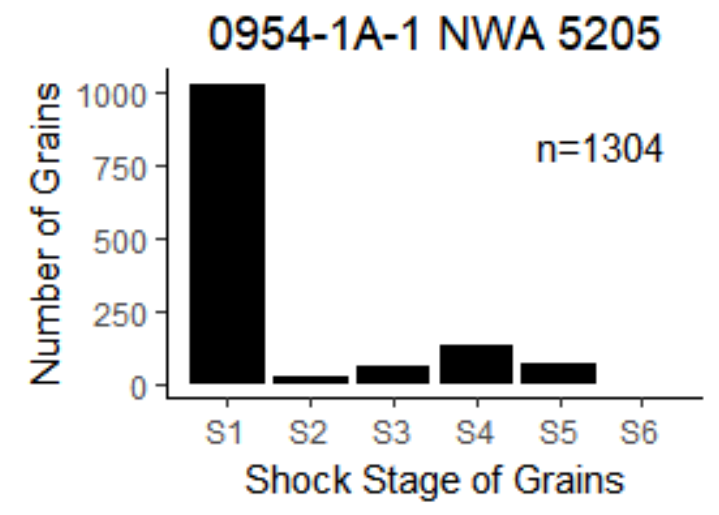

Figure 6. Grain Shock Stage Histogram of Sample CML 0954-1A-1. Shock stage is as defined by Stöffler et al. (1991, 2018).

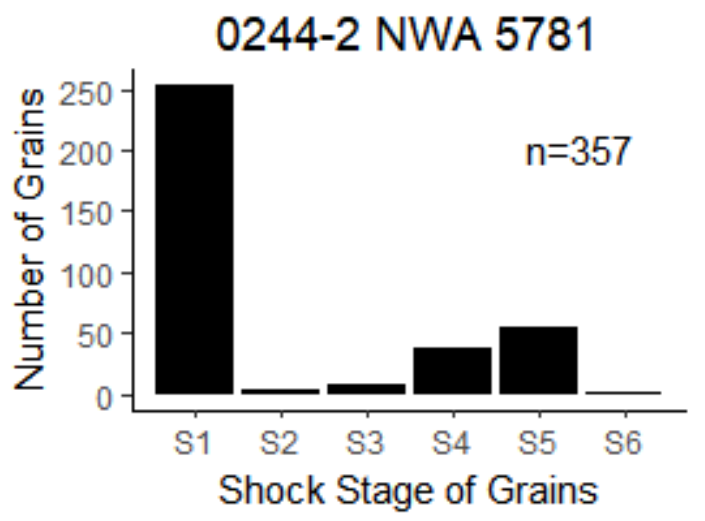

Figure 8. Grain Shock Stage Histogram of Sample CML 0244-2. Shock stage is as defined by Stöffler et al. (1991, 2018).

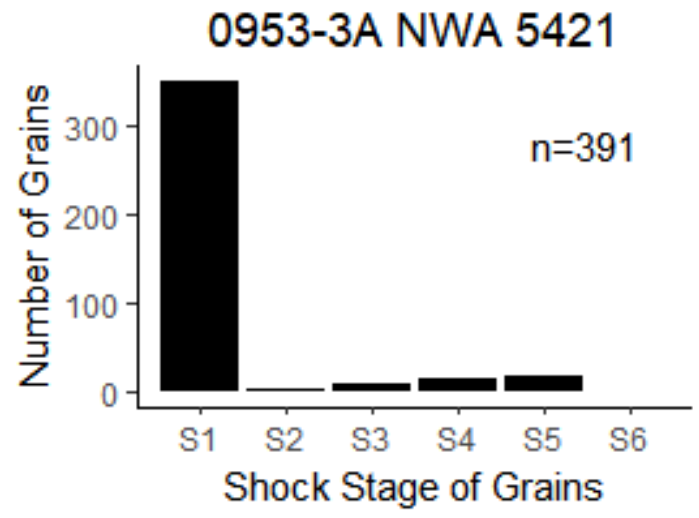

Figure 7. Grain Shock Stage Histogram of Sample CML 0953-3A. Shock stage is as defined by Stöffler et al. (1991, 2018).

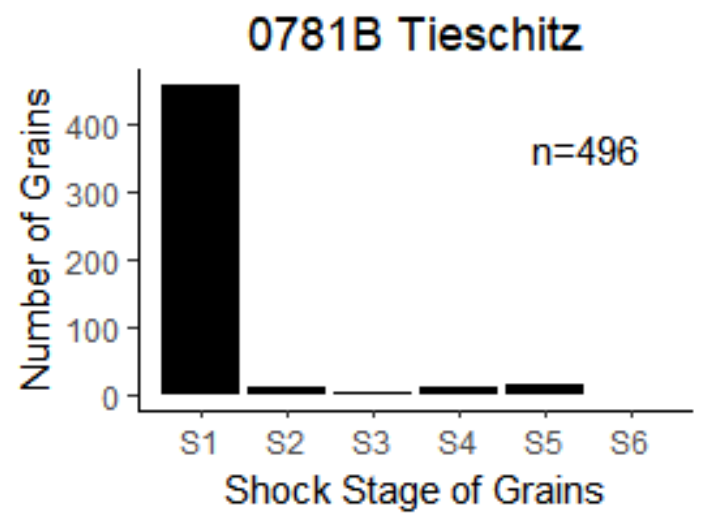

Figure 9. Grain Shock Stage Histogram of Sample CML 0781B. Shock stage is as defined by Stöffler et al. (1991, 2018). 
0954-1A-1 NWA 5205

\begin{tabular}{|c|c|c|c|c|c|c|c|}
\hline 9 & 28 & 26 & 29 & 39 & 33 & 4 & \multicolumn{1}{|c}{} \\
\hline 29 & 23 & 44 & 50 & 53 & 36 & 46 & 8 \\
\hline 48 & 32 & 19 & 28 & 19 & 49 & 22 & 23 \\
\hline 26 & 18 & 28 & 50 & 22 & 15 & 37 & 26 \\
\hline 36 & 20 & 34 & 30 & 27 & 37 & 25 & 10 \\
\hline 28 & 25 & 20 & 16 & 20 & 29 & 22 & 6 \\
\hline
\end{tabular}
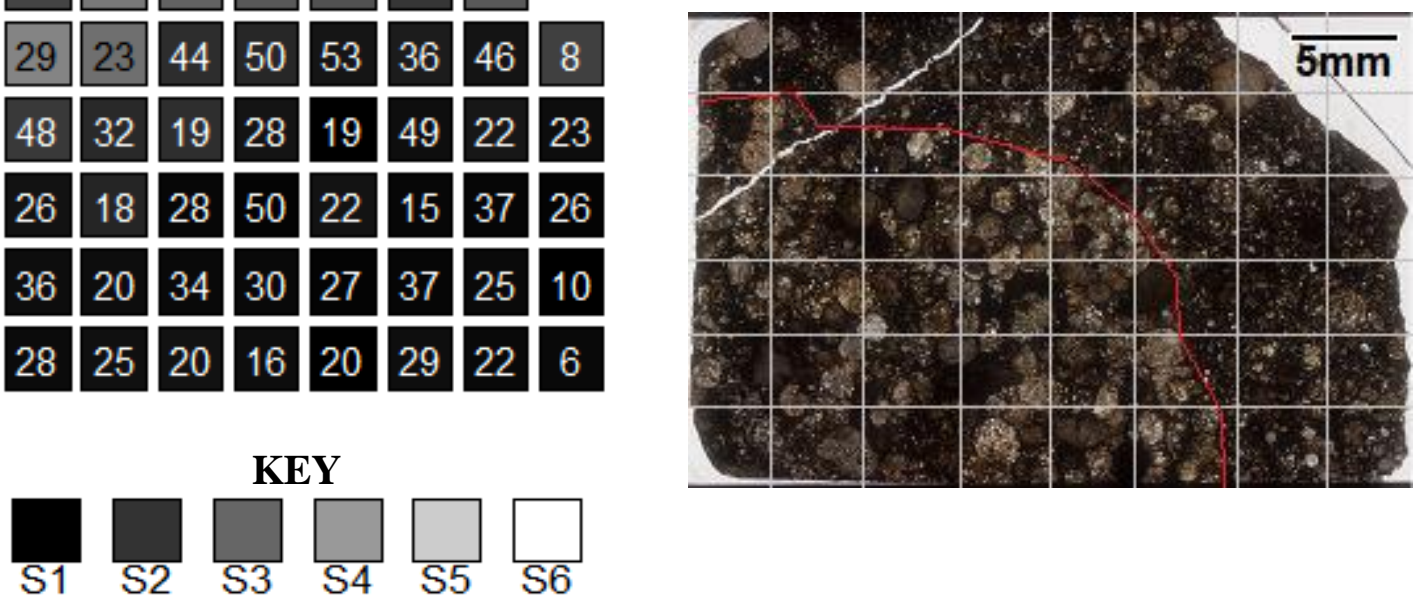

Figure 10. Shock Map of Sample CML 0954-1A-1. Left image is a sectored shock map, and the right is an image of the sample in the same orientation. Numbers in the shock map sectors are the number of measured grains in those cells. Sector color is based on the weighted shock stage of a sector on the scale defined by the key. Red line denotes boundary of cluster chondrite clast, which occupies the lower left of the thin-section image. 
0953-3A NWA 5421
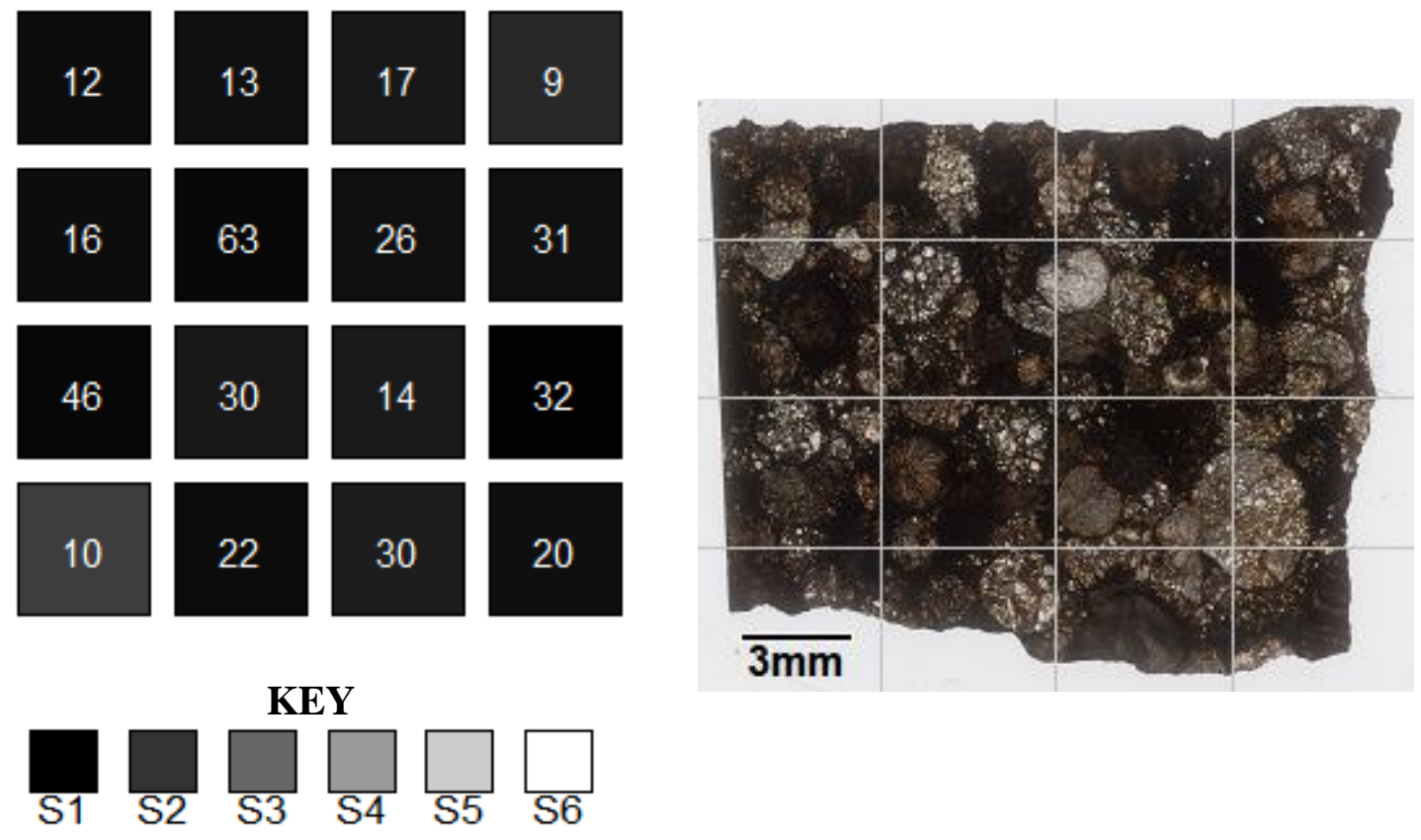

Figure 11. Shock Map of Sample CML 0953-3A. Left image is a sectored shock map, and the right is an image of the sample in the same orientation. Numbers in the shock map sectors are the number of measured grains in those cells. Sector color is based on the weighted shock stage of a sector on the scale defined by the key. 
0244-2 NWA 5781
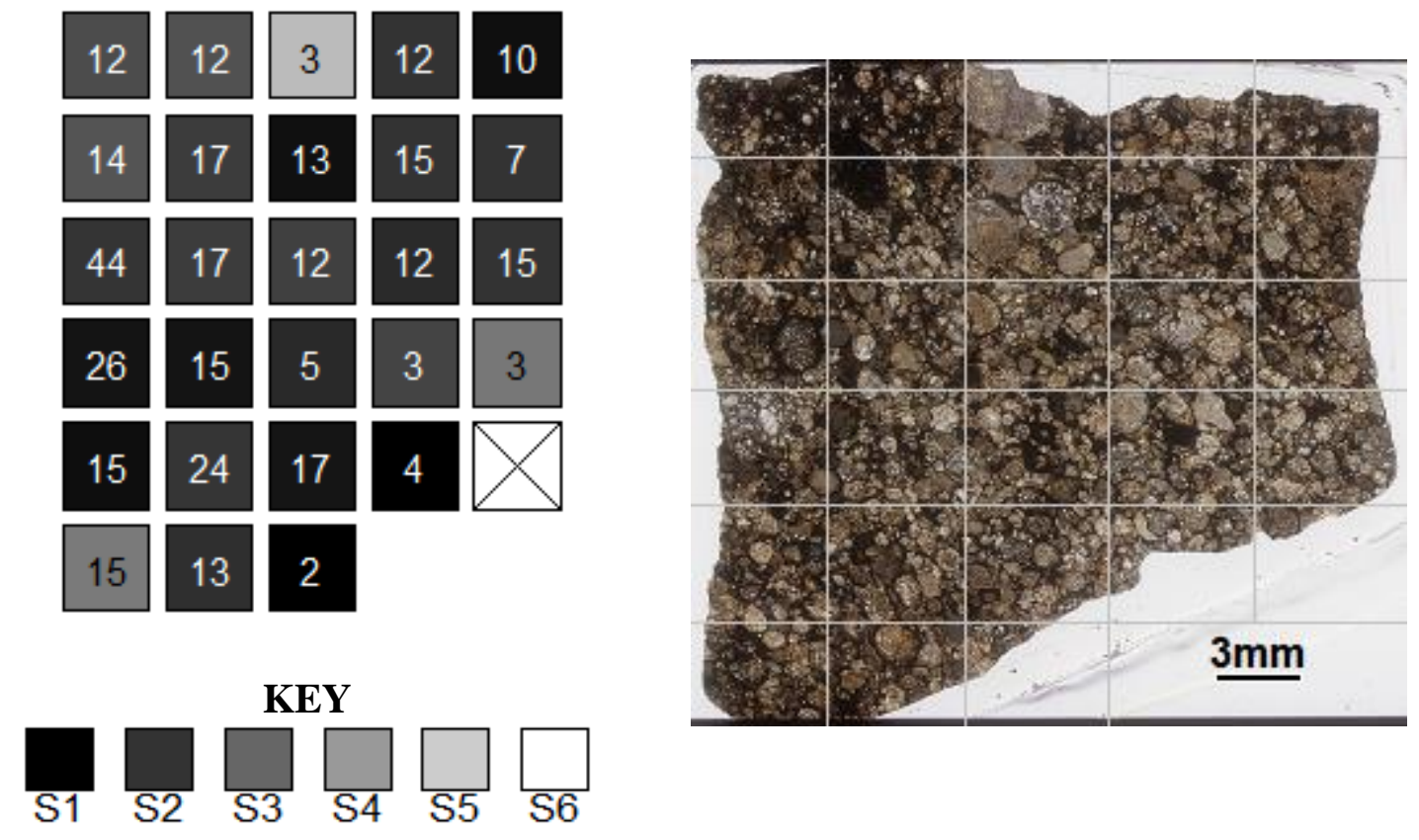

Figure 12. Shock Map of Sample CML 0244-2. Left image is a sectored shock map, and the right is an image of the sample in the same orientation. Numbers in the shock map sectors are the number of measured grains in those cells. Sector color is based on the weighted shock stage of a sector on the scale defined by the key. Sectors with an $\mathrm{X}$ have no grains usable for shock stage assessment.

\section{B Tieschitz}
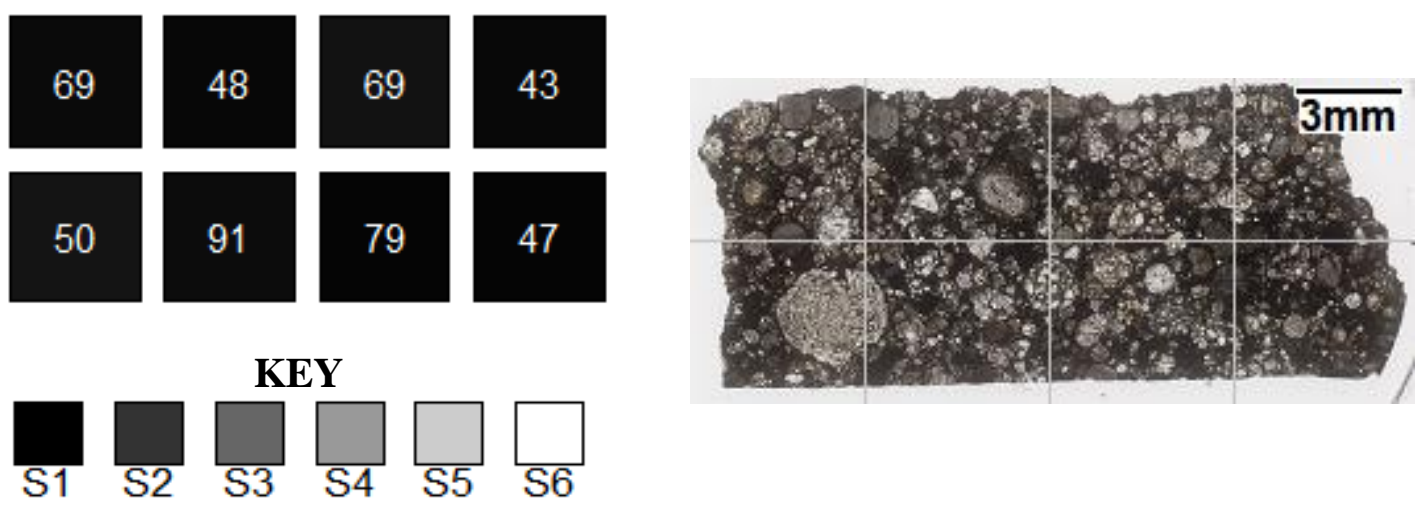

Figure 13. Shock Map of Sample CML 0781B. Left image is a sectored shock map, and the right is an image of the sample in the same orientation. Numbers in the shock map sectors are the number of measured grains in those cells. Sector color is based on the weighted shock stage of a sector on the scale defined by the key. 


\subsection{EBSD Area Map Results}

Tables 4 and 5 display data concerning the EBSD maps collected. Annealing parameters for the samples are displayed in Table 6. EBSD maps of the analyzed samples found generally low annealing parameters for the samples intermediate to the unannealed and annealed values documented by Ruzicka and Hugo (2018). The notable exception is Tieschitz, which has an annealing parameter consistent with metamorphic annealing. Figure 14 shows the locations of the mapped areas in the samples. Figures 15-22, 23-30, and 31-38 respectively display GOS, local misorientation, and grain orientation maps of the EBSD rastered areas. Figures 39-42 display CRA stereonets for the EBSD mapped regions of the four analyzed samples, showing activation of both a-slip and c-slip type systems in all samples. Figure 43 presents the analyzed samples plotted in relation to weighted shock stage and GOS/MOS relationships documented by Ruzicka and Hugo (2018), showing that the samples plot in a cluster removed from the established trendline. Figure 44 displays the apparent annealing and temperature parameters of the analyzed EBSD maps to those of Ruzicka and Hugo (2018). Figure 45 shows the modal slip system activation of the studied samples. 
Table 4. Metadata for EBSD Area Maps. "SAM" denotes "small area map," representing EBSD mosaics at finer step sizes and smaller mapped areas. "LAM" denotes "large area map," representing EBSD mosaics at coarser step sizes and covering larger areas. Step size is the distance between pixels in the mapped mosaic. "GOS" refers to GOS values of olivine grains greater than $50 \mu \mathrm{m}$ in diameter. Map names are derived from their date of collection, in the format MMDD.

\begin{tabular}{|c|c|c|c|c|c|c|c|}
\hline Sample & Map & Type & $\begin{array}{l}\begin{array}{l}\text { Area } \\
\left(\mathbf{m m}^{2}\right)\end{array} \\
\end{array}$ & $\begin{array}{l}\text { Subset Size } \\
\left(\mathbf{m m}^{2}\right)\end{array}$ & $\begin{array}{l}\text { Step Size } \\
(\mu \mathrm{m})\end{array}$ & $\begin{array}{l}\text { Total } \\
\text { Pixels } \\
\end{array}$ & $\begin{array}{l}\text { Olivine } \\
\text { Grains }\end{array}$ \\
\hline \multicolumn{8}{|l|}{$0244-2$} \\
\hline \multicolumn{8}{|l|}{ NWA } \\
\hline 5781 & 0923 & SAM & 10.9 & 2.18 & 1.99 & $2,748,603$ & 181 \\
\hline \multicolumn{8}{|l|}{$0244-2$} \\
\hline \multicolumn{8}{|l|}{ NWA } \\
\hline 5781 & 1005 & LAM & 97.6 & 19.5 & 4.00 & $6,087,400$ & 1336 \\
\hline \multicolumn{8}{|l|}{ 0781B } \\
\hline Tieschitz & 0208 & LAM & 66.4 & 13.3 & 3.99 & $4,152,588$ & 1280 \\
\hline \multicolumn{8}{|l|}{ 0781B } \\
\hline Tieschitz & 0215 & LAM & 53.1 & 10.6 & 3.62 & $4,030,983$ & 920 \\
\hline \multicolumn{8}{|l|}{ 0953-3A } \\
\hline \multicolumn{8}{|l|}{ NWA } \\
\hline 5421 & 1215 & SAM & 20.6 & 4.12 & 2.60 & $3,040,052$ & 478 \\
\hline \multicolumn{8}{|l|}{ 0953-3A } \\
\hline \multicolumn{8}{|l|}{ NWA } \\
\hline 5421 & 1222 & LAM & 75.9 & 15.2 & 4.00 & $4,742,080$ & 1427 \\
\hline \multicolumn{8}{|l|}{ 0954-1A-1 } \\
\hline \multicolumn{8}{|l|}{ NWA } \\
\hline 5205 & 0118 & SAM & 25.8 & 5.16 & 3.48 & $2,123,454$ & 641 \\
\hline \multicolumn{8}{|l|}{ 0954-1A-1 } \\
\hline NWA & & & & & & & \\
\hline 5205 & 0201 & LAM & 155 & 31.0 & 5.00 & $6,172,551$ & 1940 \\
\hline
\end{tabular}


NWA 5205

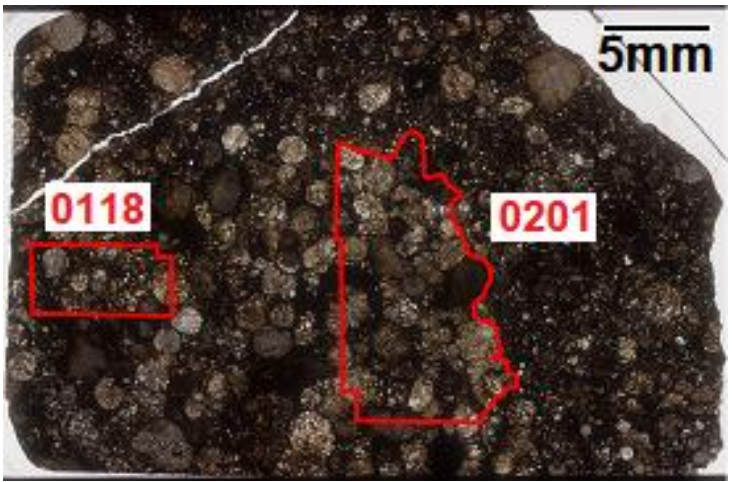

NWA 5781

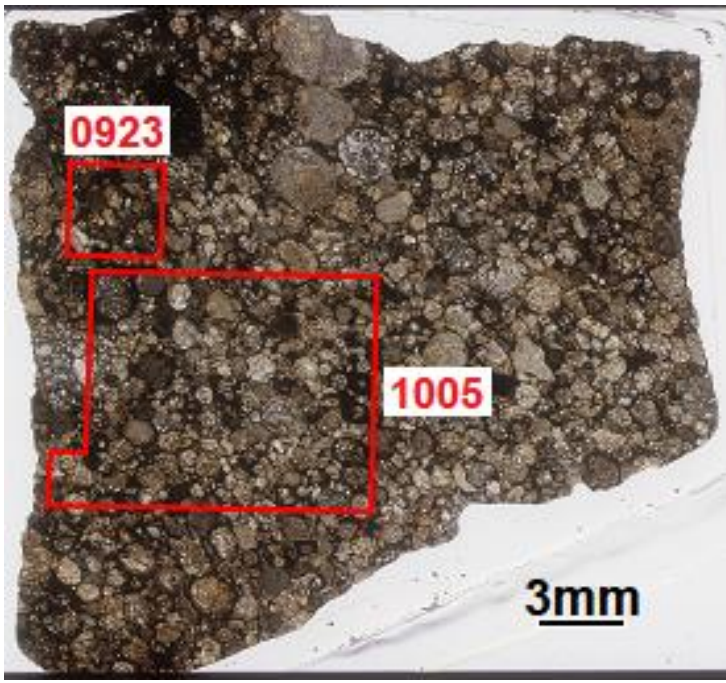

NWA 5421

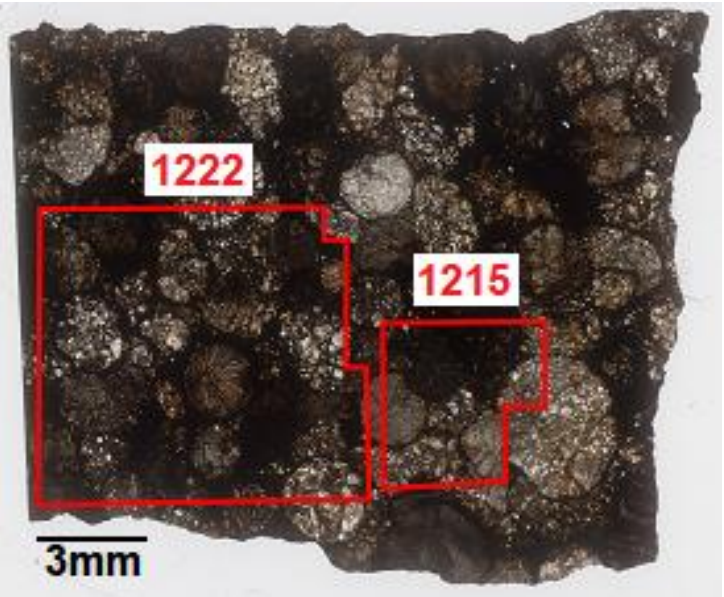

Tieschitz

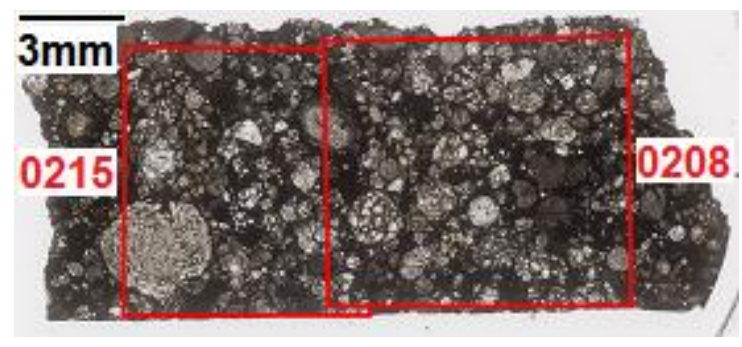

Figure 14. EBSD Mapped Areas of Selected Samples. Mapped areas are labeled by their date of acquisition (MMDD). All have one SAM and one LAM, except for Tieschitz, which has two LAMs. LAM areas are larger than SAM areas, but have larger raster step sizes. 
Table 5. EBSD Area Maps Results. Regional subsets are vertical or horizontal strips covering $20 \%$ of an EBSD mosaic. "GOS" and "MOS" refer to GOS and MOS values of olivine grains greater than $50 \mu \mathrm{m}$ in diameter. $\left.f_{\langle 010\rangle}+f_{\langle 001}\right\rangle$ is the sum of the modal pole densities of the two a-slip systems in CRA stereonets; it is synonymous with the temperature parameter. Map names are derived from their date of collection, in the format MMDD.

\begin{tabular}{|c|c|c|c|c|c|c|c|}
\hline Sample & Map & $\begin{array}{l}\text { GOS } \\
\text { Mean }\left({ }^{\circ}\right) \\
\end{array}$ & $\begin{array}{l}\text { GOS Std. } \\
\text { Dev. }\left({ }^{\circ}\right)\end{array}$ & $\begin{array}{l}\text { GOS } \\
\text { Mean/Med. }\left({ }^{\circ}\right)\end{array}$ & $\begin{array}{l}\text { MOS } \\
\text { Mean }\left({ }^{\circ}\right)\end{array}$ & $\begin{array}{l}\text { MOS Std. } \\
\text { Dev. }\left({ }^{\circ}\right) \\
\end{array}$ & $\begin{array}{l}f_{<010>+}+ \\
f_{\langle 001>} \\
\end{array}$ \\
\hline \multicolumn{8}{|l|}{$0244-2$} \\
\hline NWA 5781 & 0923 & 2.69 & 1.85 & 1.16 & 14.6 & 7.35 & 0.614 \\
\hline \multicolumn{8}{|l|}{ 0244-2 } \\
\hline NWA 5781 & 1005 & 2.37 & 1.58 & 1.25 & 10.3 & 5.72 & 0.604 \\
\hline \multicolumn{8}{|l|}{ 0781B } \\
\hline Tieschitz & 0208 & 1.69 & 1.91 & 1.87 & 8.86 & 6.33 & 0.624 \\
\hline \multicolumn{8}{|l|}{ 0781B } \\
\hline Tieschitz & 0215 & 1.44 & 1.91 & 2.15 & 8.03 & 6.69 & 0.640 \\
\hline \multicolumn{8}{|l|}{ 0953-3A } \\
\hline NWA 5421 & 1215 & 2.90 & 2.09 & 1.24 & 13.6 & 6.17 & 0.613 \\
\hline \multicolumn{8}{|l|}{ 0953-3A } \\
\hline NWA 5421 & 1222 & 2.60 & 2.00 & 1.24 & 12.4 & 6.33 & 0.617 \\
\hline \multicolumn{8}{|l|}{ 0954-1A-1 } \\
\hline NWA 5205 & 0118 & 2.18 & 1.95 & 1.36 & 11.7 & 7.88 & 0.592 \\
\hline \multicolumn{8}{|l|}{ 0954-1A-1 } \\
\hline NWA 5205 & 0201 & 2.43 & 1.85 & 1.26 & 10.7 & 5.87 & 0.619 \\
\hline
\end{tabular}

Table 6. Apparent Annealing Parameters of EBSD Analyzed Samples. Annealing parameter is (mean GOS of $>50 \mu \mathrm{m}$ olivine grains) / (median GOS of $>50 \mu \mathrm{m}$ olivine grains). Data used is of the merged olivine grain populations from both maps of each sample. Annealed specimens from Ruzicka and Hugo (2018) have an average annealing parameter of 1.89, and non-annealed specimens have an average annealing parameter of 1.10. Studied samples have minor apparent annealing, except for Tieschitz.

\begin{tabular}{lcc}
\hline Sample & Mean GOS / Median GOS & Grains Analyzed \\
\hline 0244-2 NWA 5781 & 1.24 & 1517 \\
0781B Tieschitz & 1.98 & 2200 \\
0953-3A NWA 5421 & 1.24 & 1905 \\
0954-1A-1 NWA 5205 & 1.27 & 2581 \\
\hline
\end{tabular}




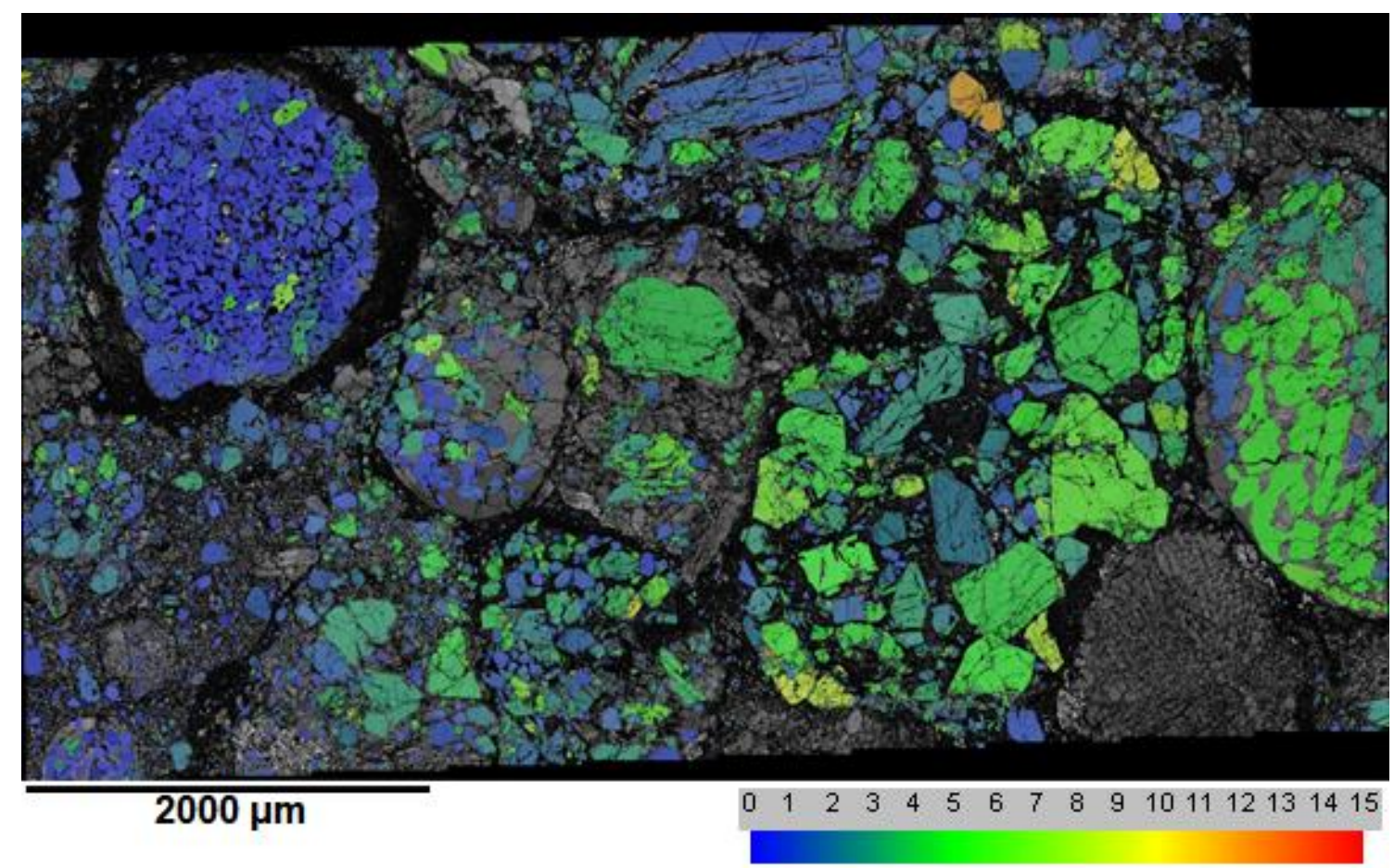

Figure 15. GOS Map 0118 of NWA 5205. Map shows olivine grains colored according to their GOS $\left(^{\circ}\right)$ values. 


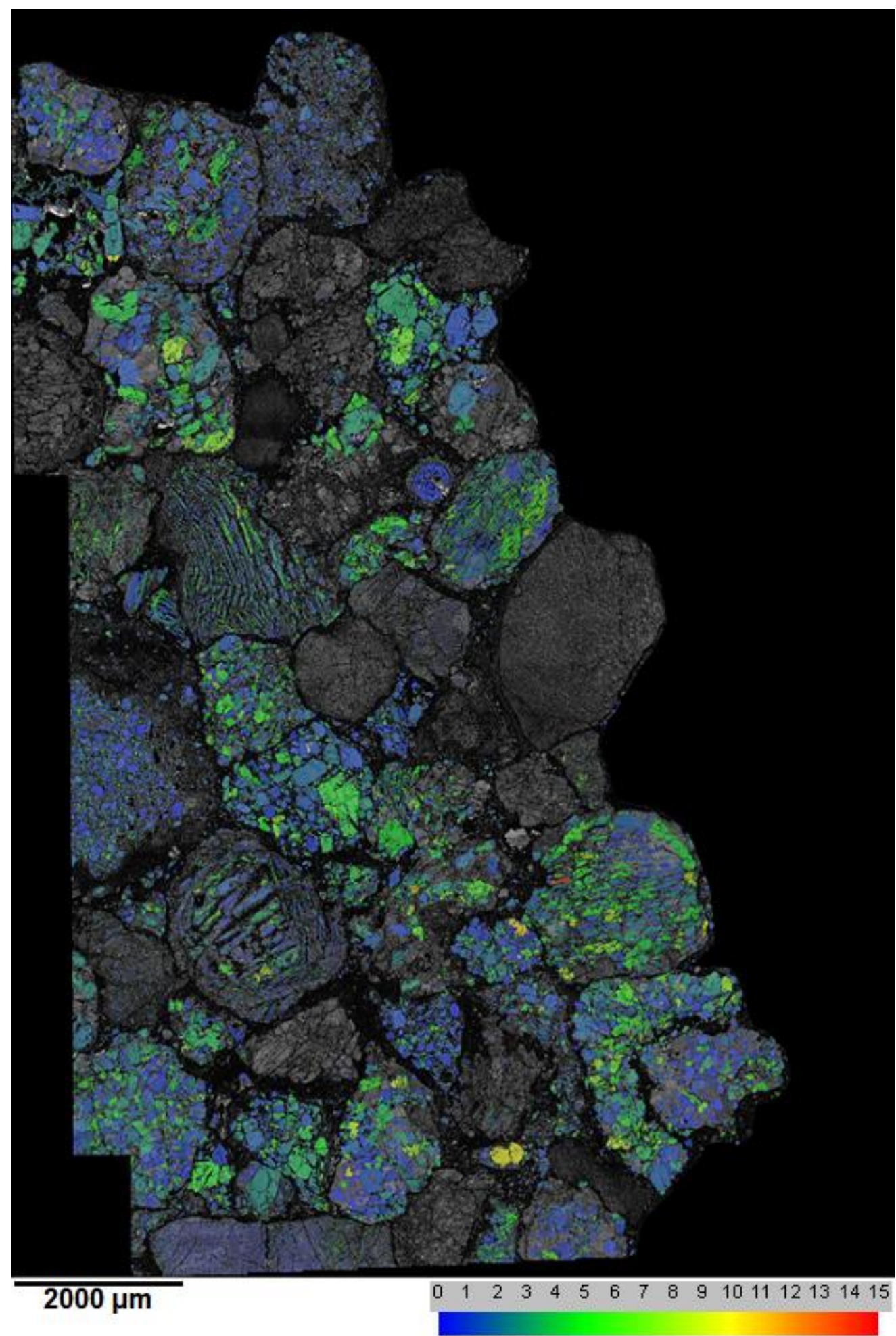

Figure 16. GOS Map 0201 of NWA 5205. Map shows olivine grains colored according to their GOS $\left(^{\circ}\right)$ values. Cluster chondrite clast only; host removed digitally. 


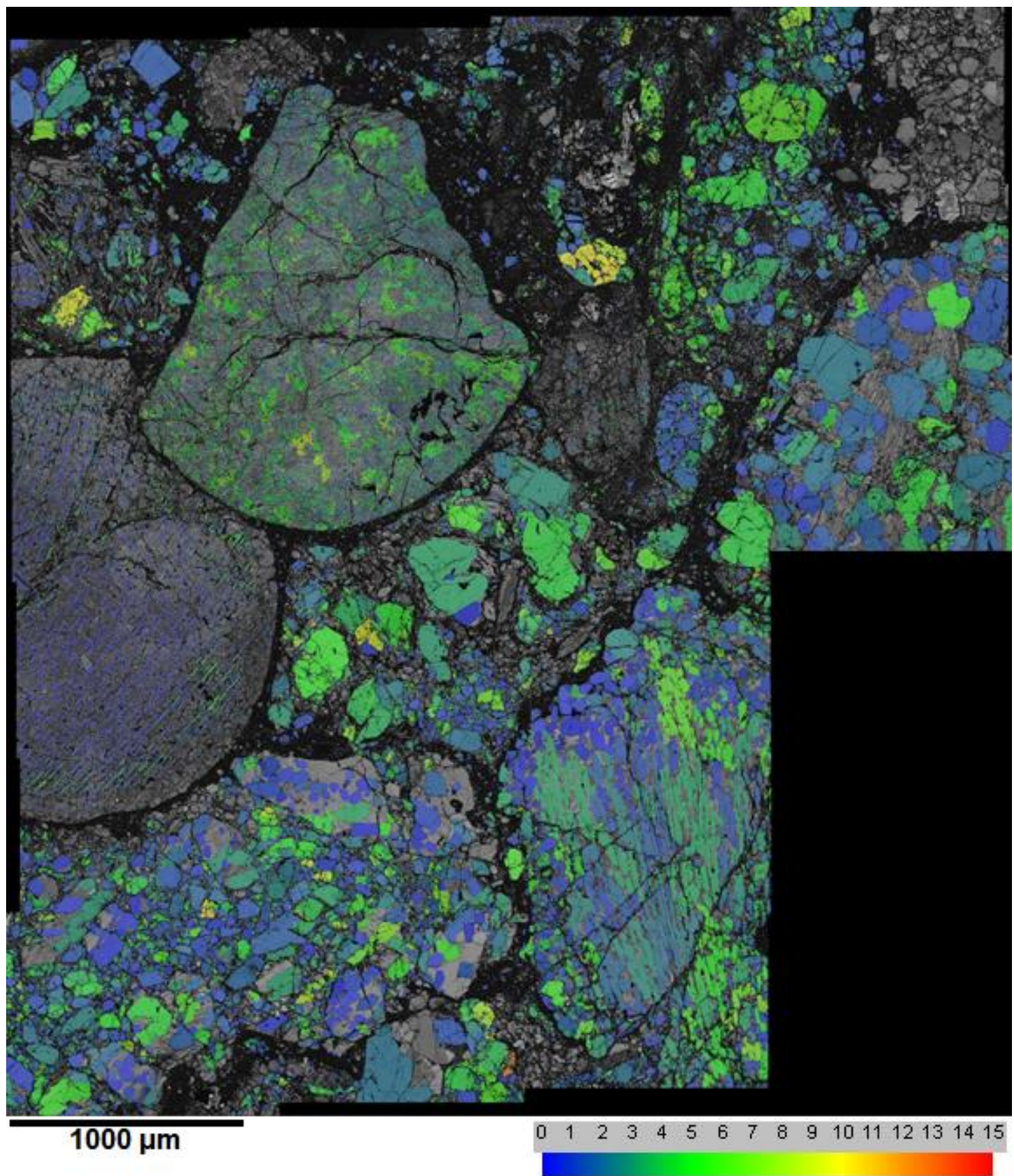

Figure 17. GOS Map 1215 of NWA 5421. Map shows olivine grains colored according to their GOS $\left({ }^{\circ}\right)$ values. 


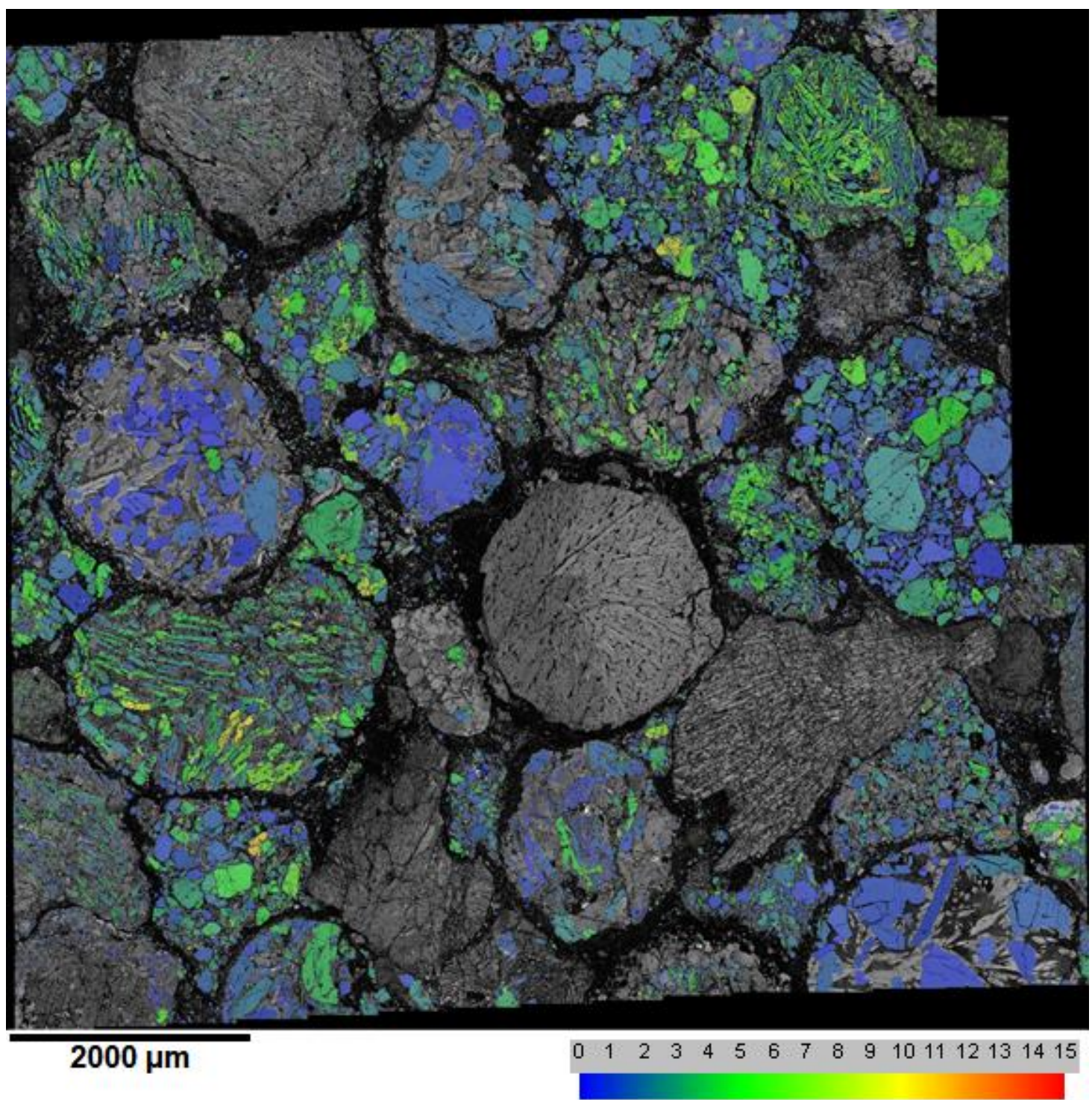

Figure 18. GOS Map 1222 of NWA 5421. Map shows olivine grains colored according to their GOS $\left(^{\circ}\right)$ values. 


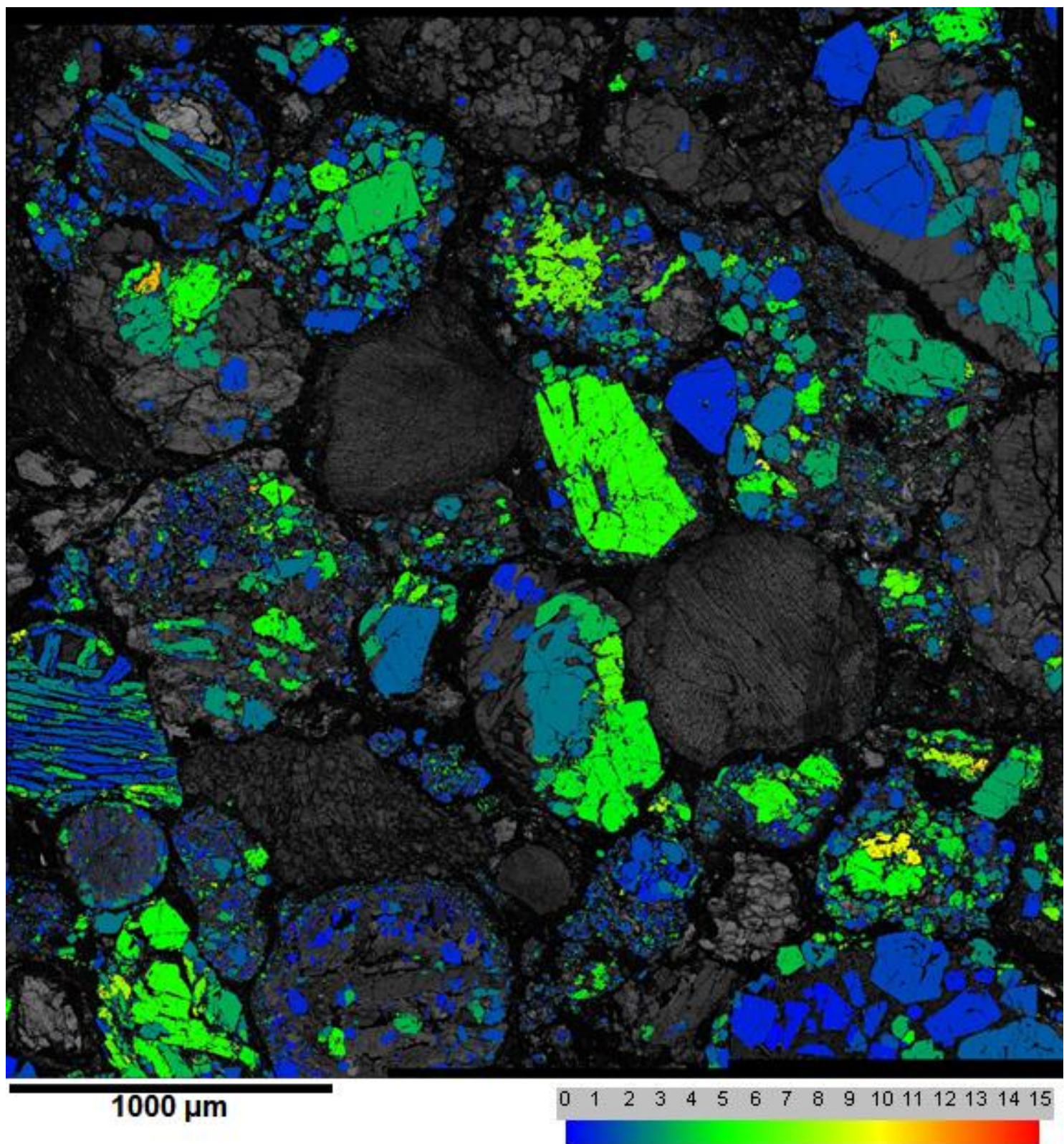

Figure 19. GOS Map 0923 of NWA 5781. Map shows olivine grains colored according to their GOS $\left({ }^{\circ}\right)$ values. 


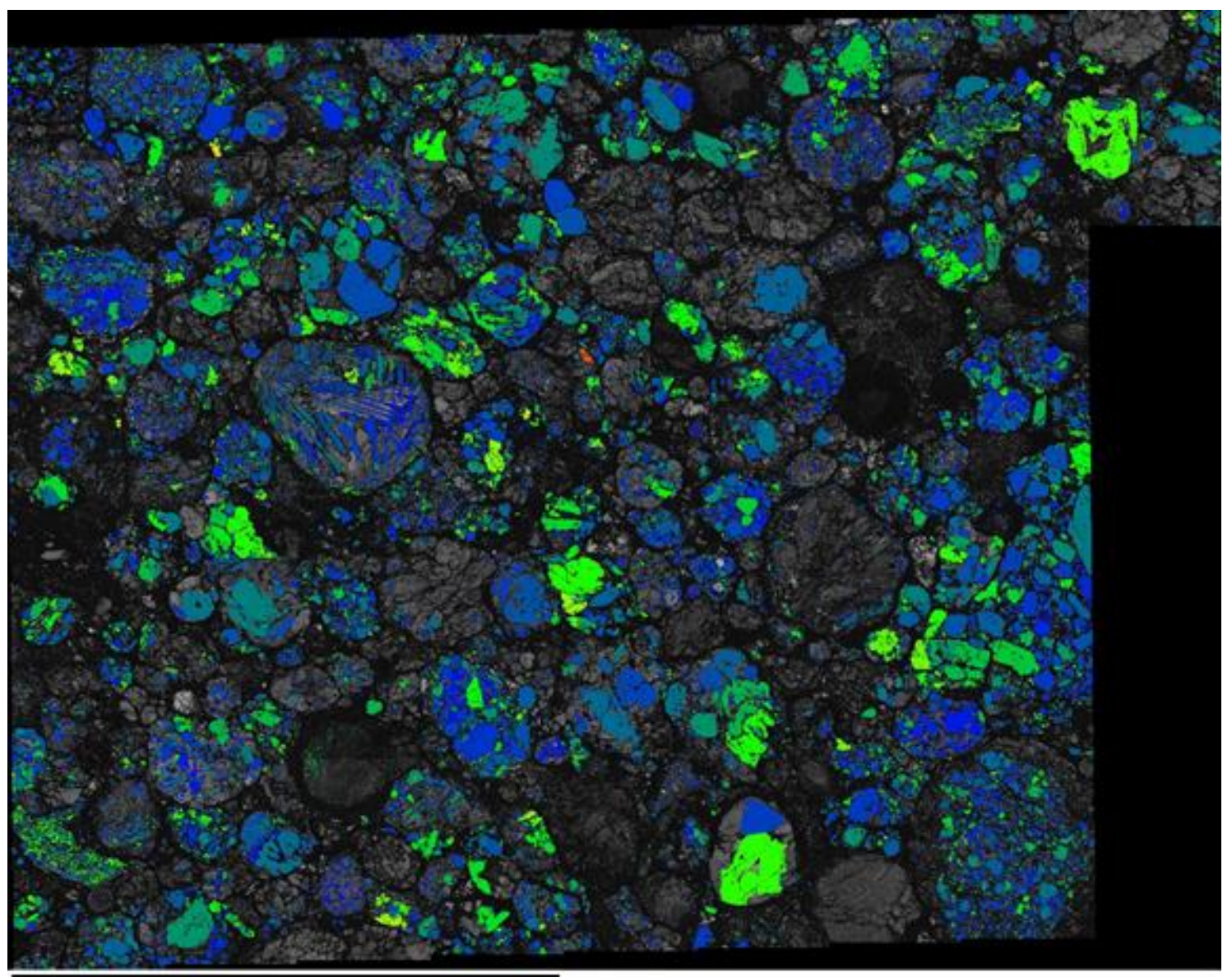

$5000 \mu \mathrm{m}$

$\begin{array}{lllllllllllllllll}0 & 1 & 2 & 3 & 4 & 5 & 6 & 7 & 8 & 9 & 10 & 11 & 1213 & 14 & 15\end{array}$

Figure 20. GOS Map 1005 of NWA 5781. Map shows olivine grains colored according to their $\operatorname{GOS}\left({ }^{\circ}\right)$ values. 


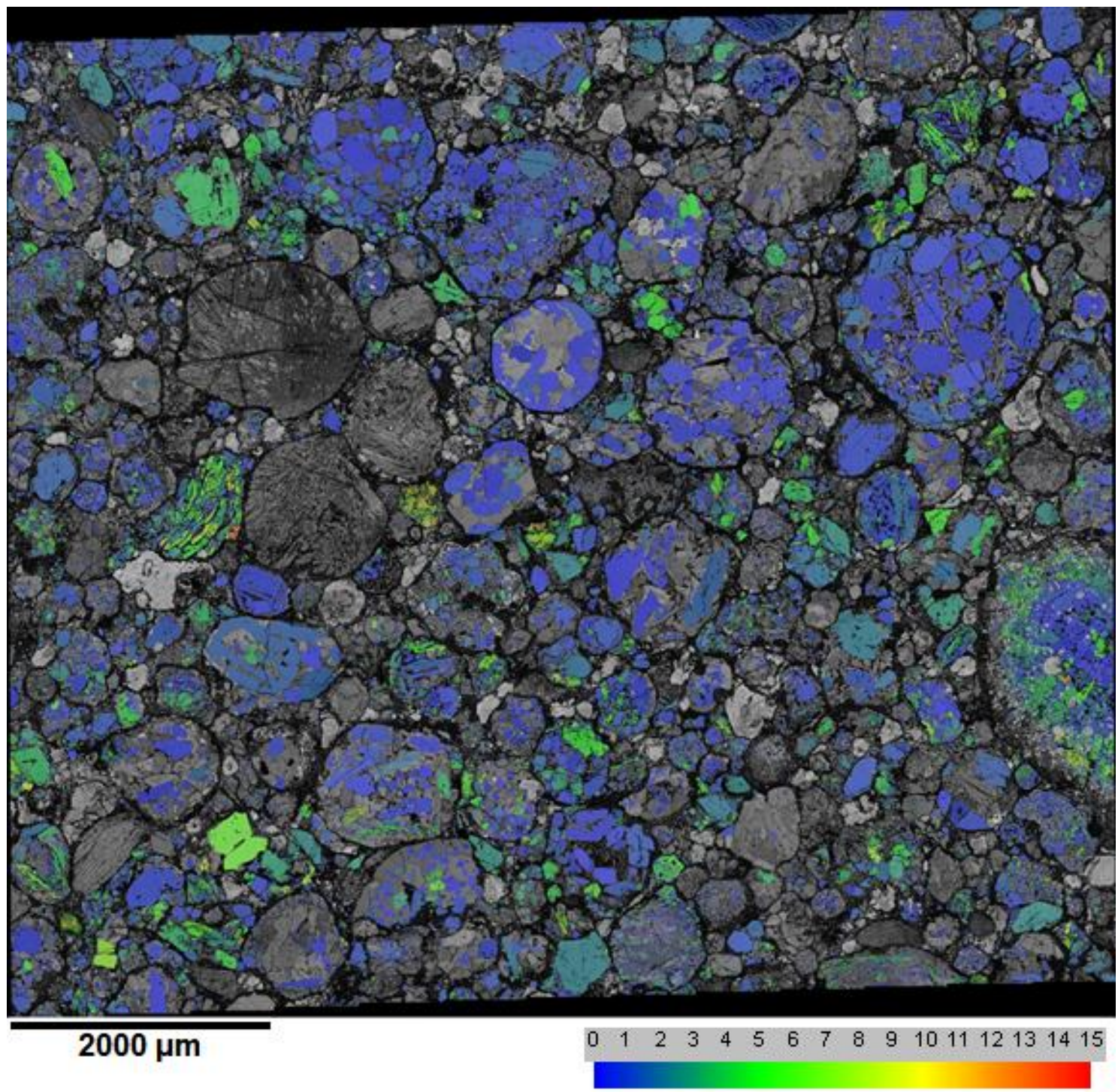

Figure 21. GOS Map 0208 of Tieschitz. Map shows olivine grains colored according to their GOS $\left(^{\circ}\right)$ values. 


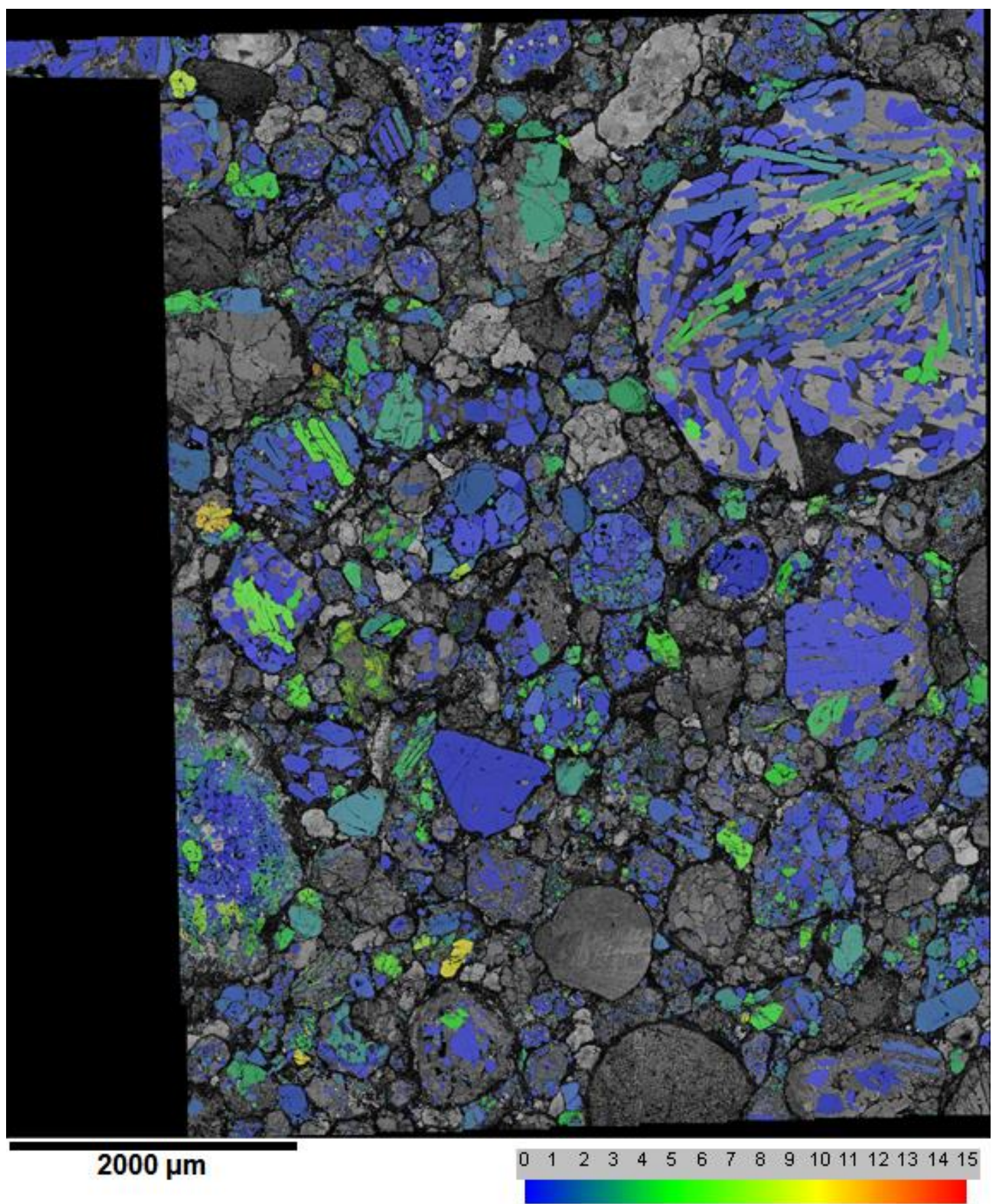

Figure 22. GOS Map 0215 of Tieschitz. Map shows olivine grains colored according to their GOS $\left(^{\circ}\right)$ values. 


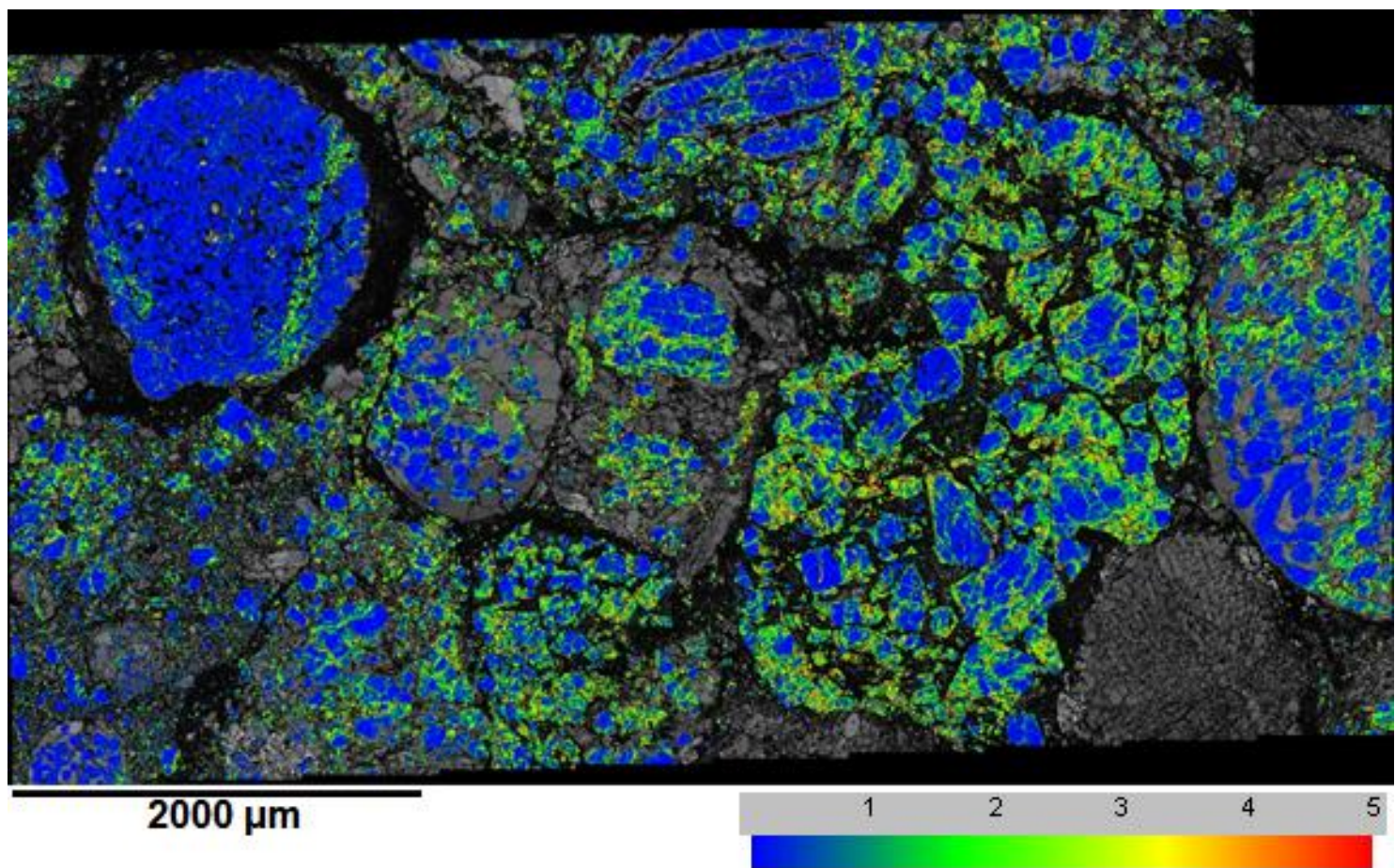

Figure 23. Local Misorientation Map 0118 of NWA 5205. Map shows local crystallographic misorientation in olivine grains, measured in degrees and displayed with a scaled color gradient. 


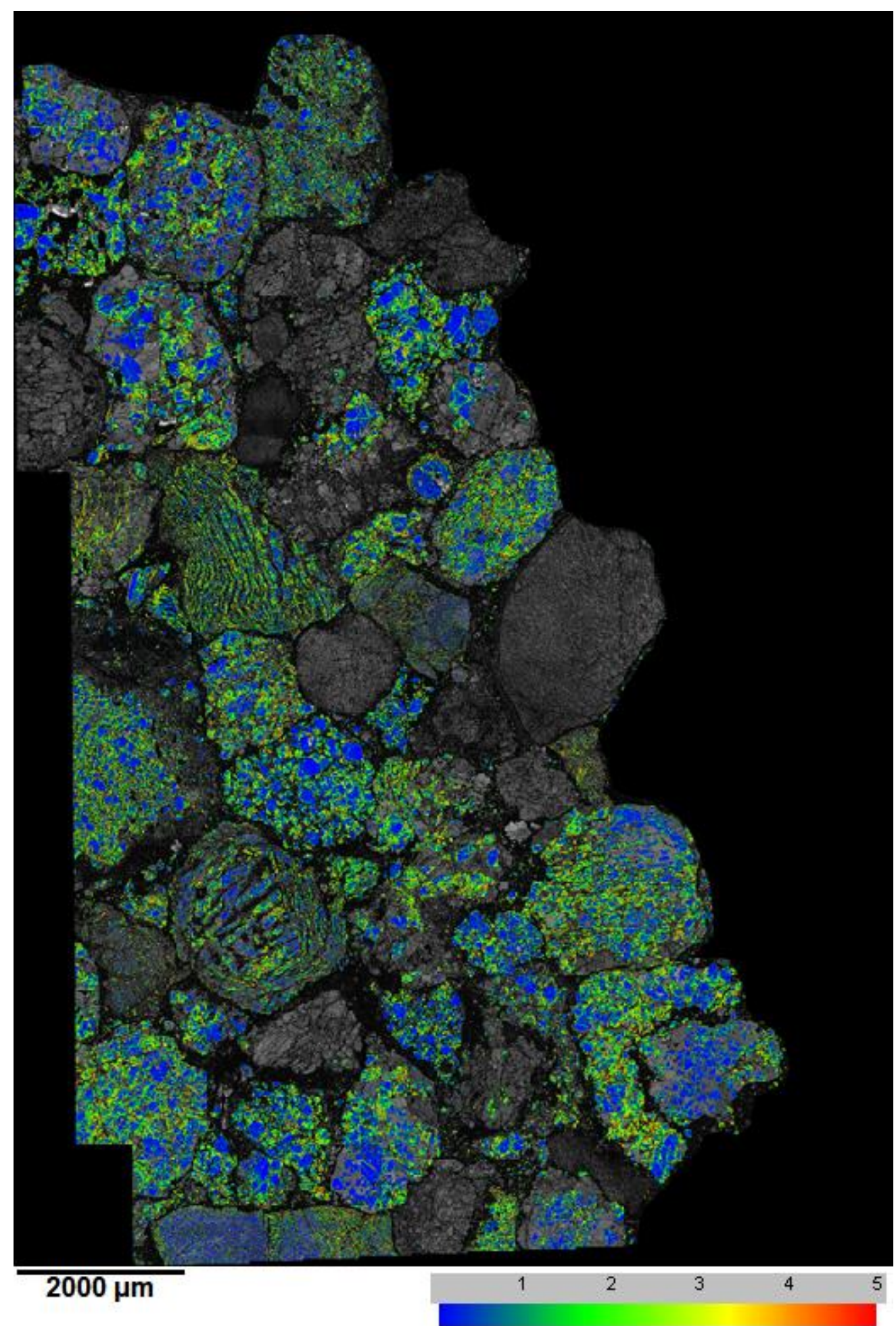

Figure 24. Local Misorientation Map 0201 of NWA 5205. Map shows local crystallographic misorientation in olivine grains, measured in degrees and displayed with a scaled color gradient. Cluster chondrite clast only; host removed digitally. 


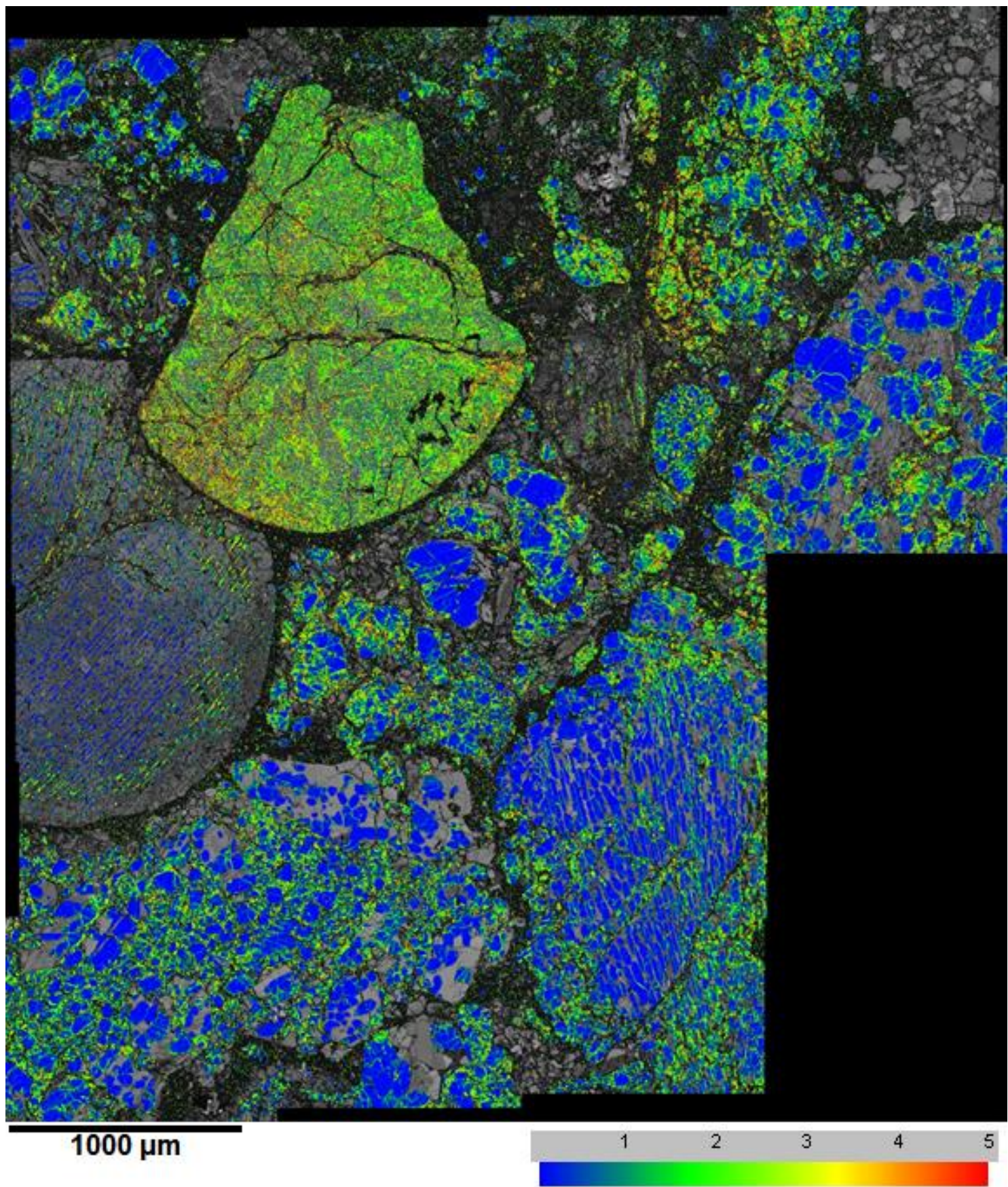

Figure 25. Local Misorientation Map 1215 of NWA 5421. Map shows local crystallographic misorientation in olivine grains, measured in degrees and displayed with a scaled color gradient. 


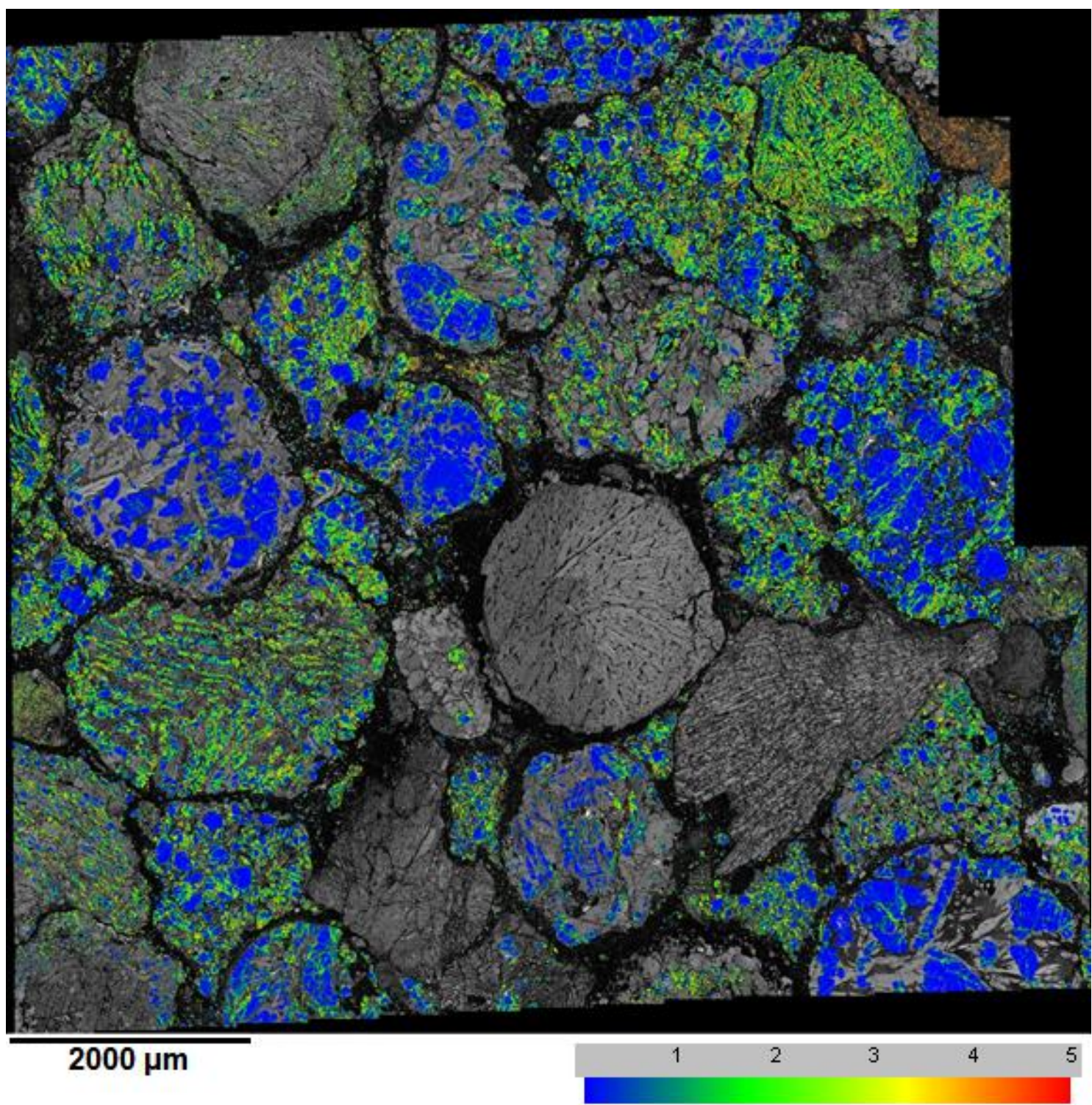

Figure 26. Local Misorientation Map 1222 of NWA 5421. Map shows local crystallographic misorientation in olivine grains, measured in degrees and displayed with a scaled color gradient. 


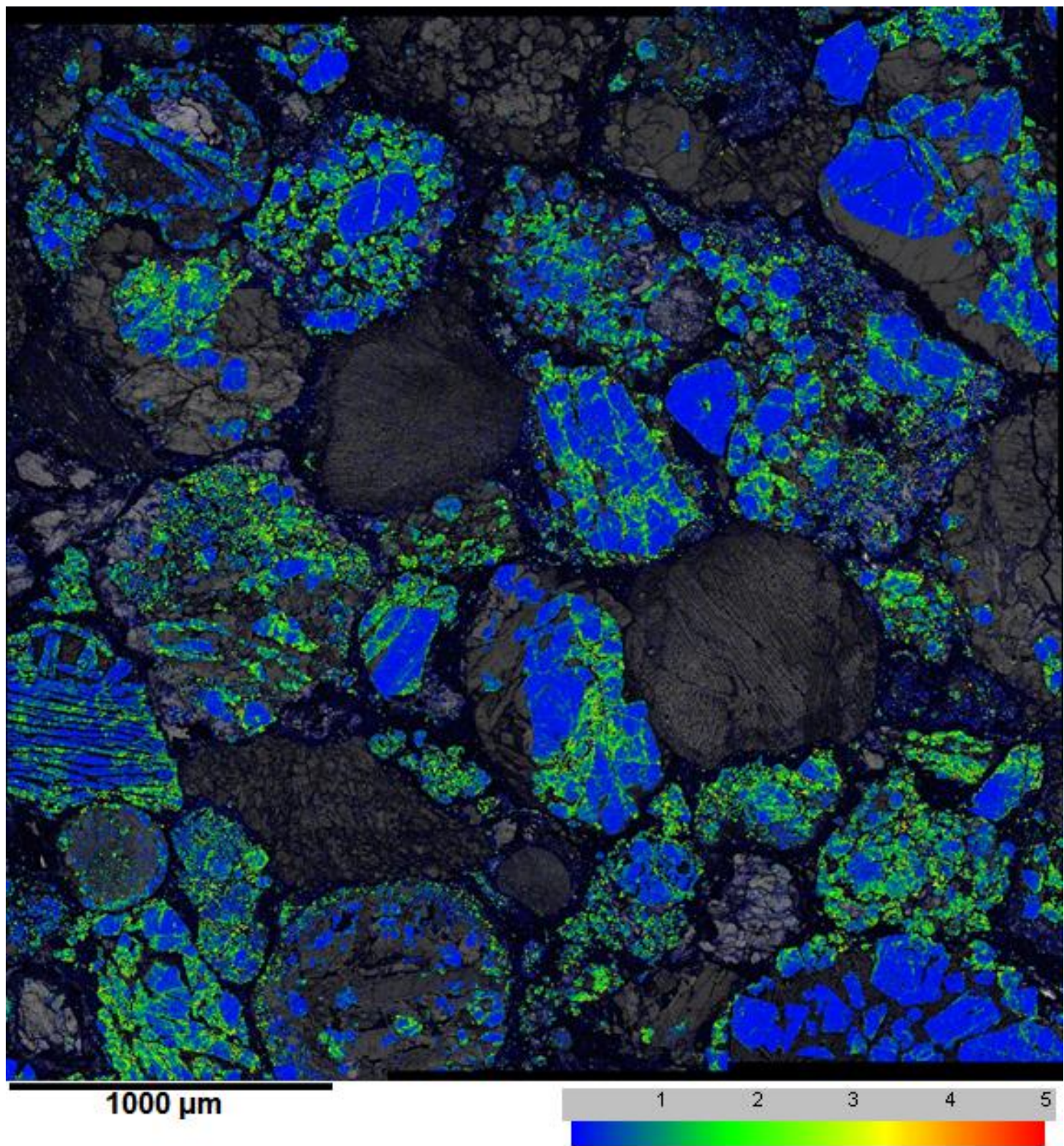

Figure 27. Local Misorientation Map 0923 of NWA 5781. Map shows local crystallographic misorientation in olivine grains, measured in degrees and displayed with a scaled color gradient. 


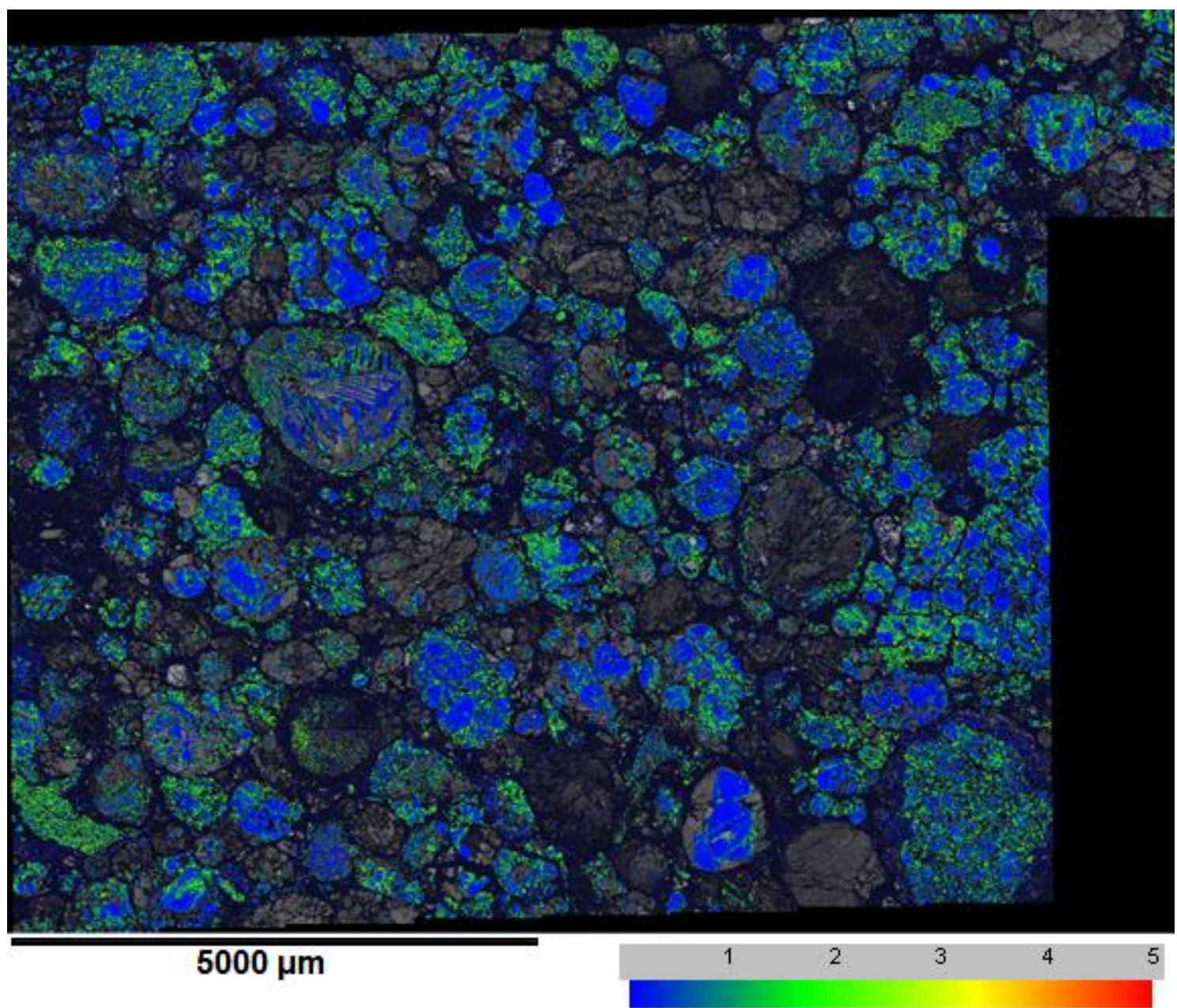

Figure 28. Local Misorientation Map 1005 of NWA 5781. Map shows local crystallographic misorientation in olivine grains, measured in degrees and displayed with a scaled color gradient. 


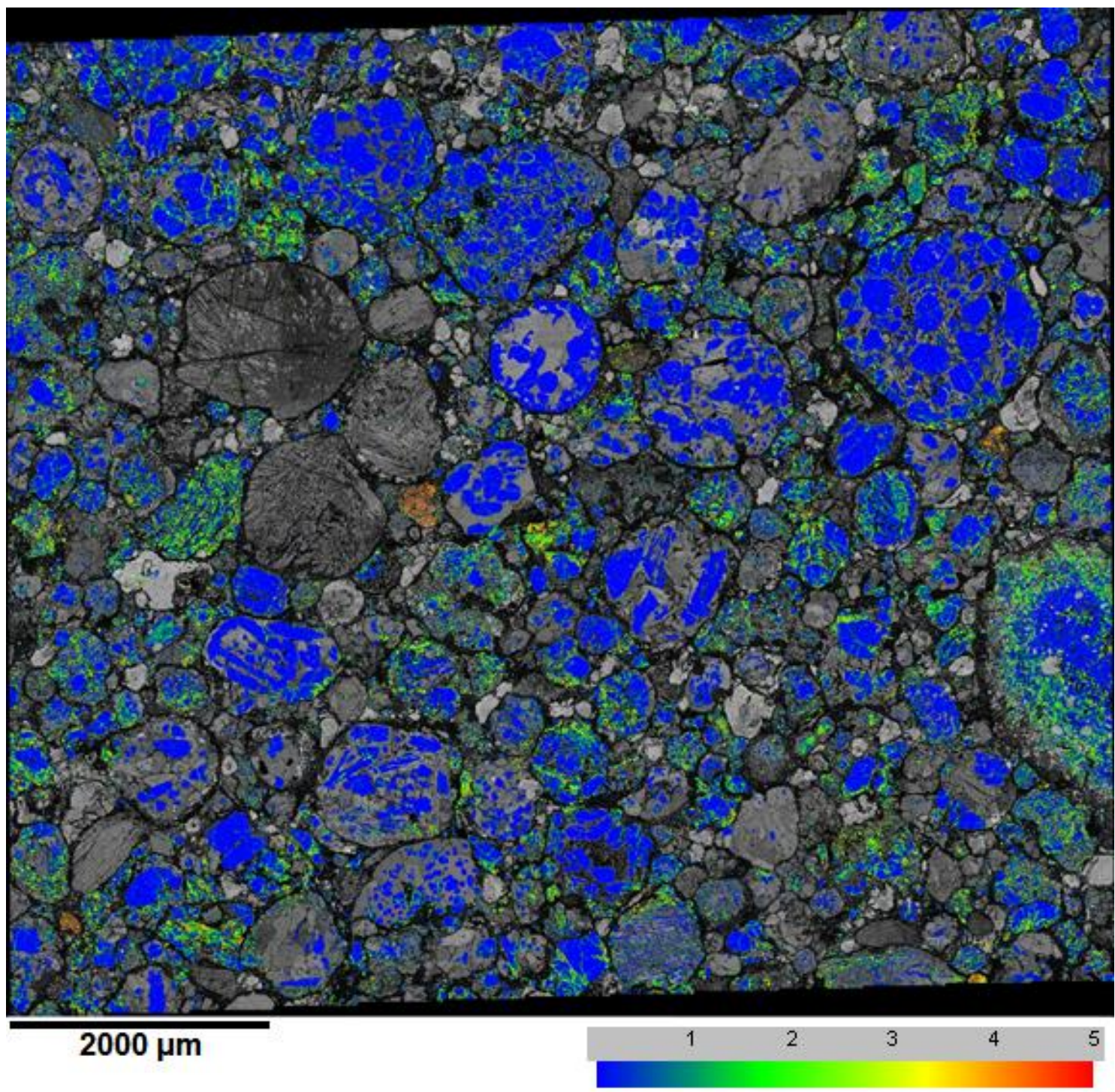

Figure 29. Local Misorientation Map 0208 of Tieschitz. Map shows local crystallographic misorientation in olivine grains, measured in degrees and displayed with a scaled color gradient. 


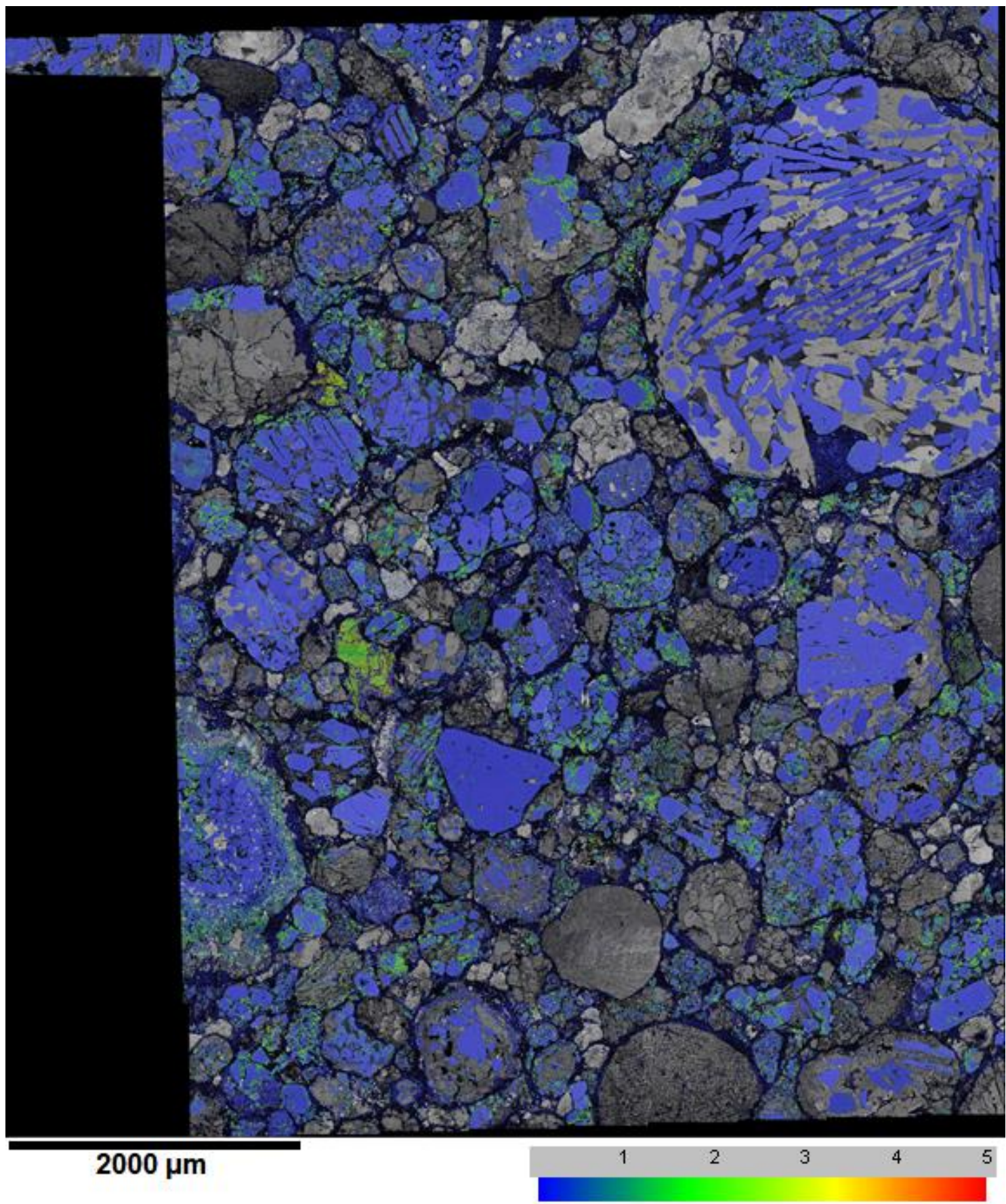

Figure 30. Local Misorientation Map 0215 of Tieschitz. Map shows local crystallographic misorientation in olivine grains, measured in degrees and displayed with a scaled color gradient. 


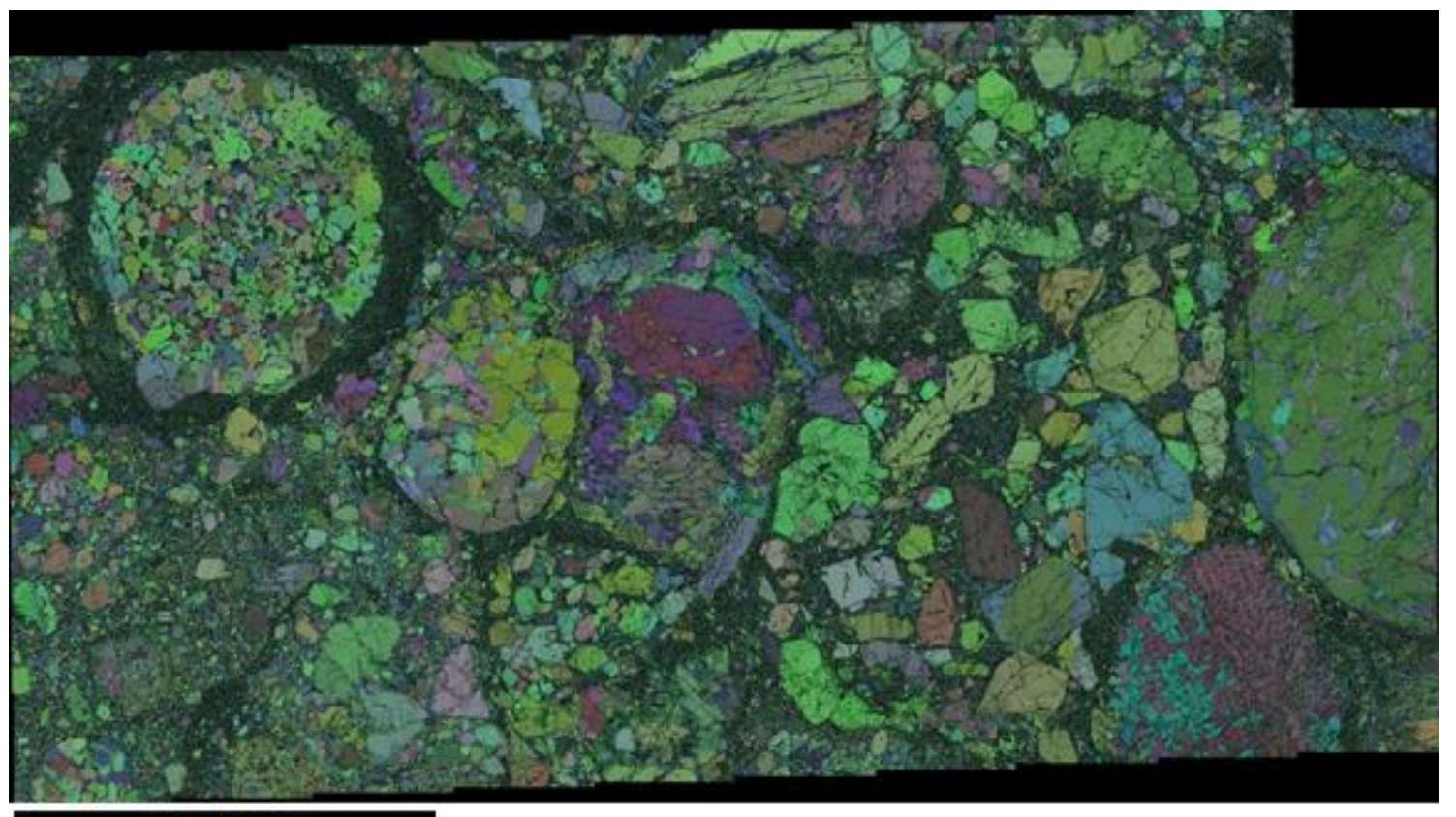

$$
2000 \mu \mathrm{m}
$$

Figure 31. Euler Map 0118 of NWA 5205. Map shows orientation of all crystal grains of all phases in a Euler coordinate system, as indicated by the color of the grains. 


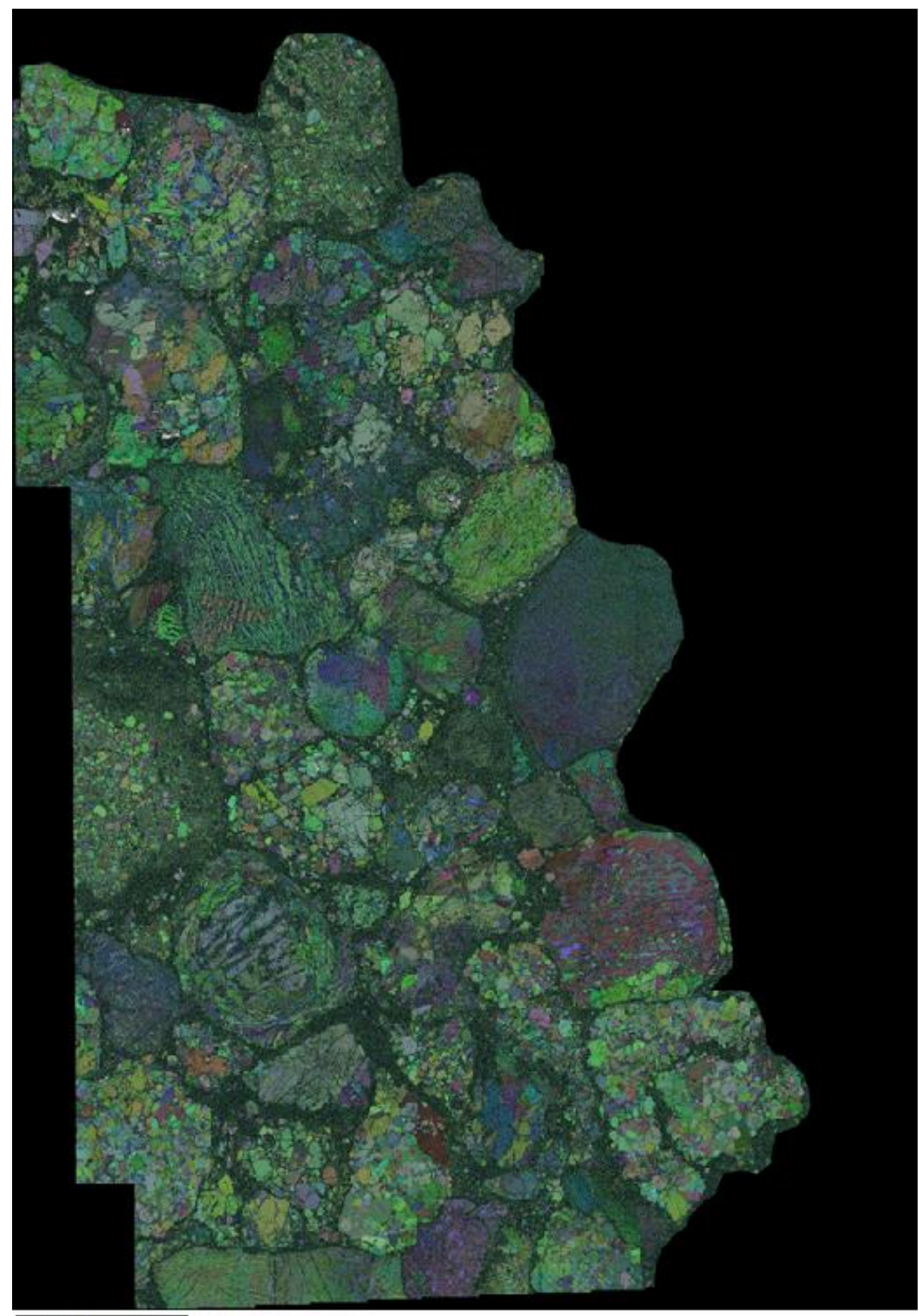

$2000 \mu \mathrm{m}$

Figure 32. Euler Map 0201 of NWA 5205. Map shows orientation of all crystal grains of all phases in a Euler coordinate system, as indicated by the color of the grains. Cluster chondrite clast only; host removed digitally. 


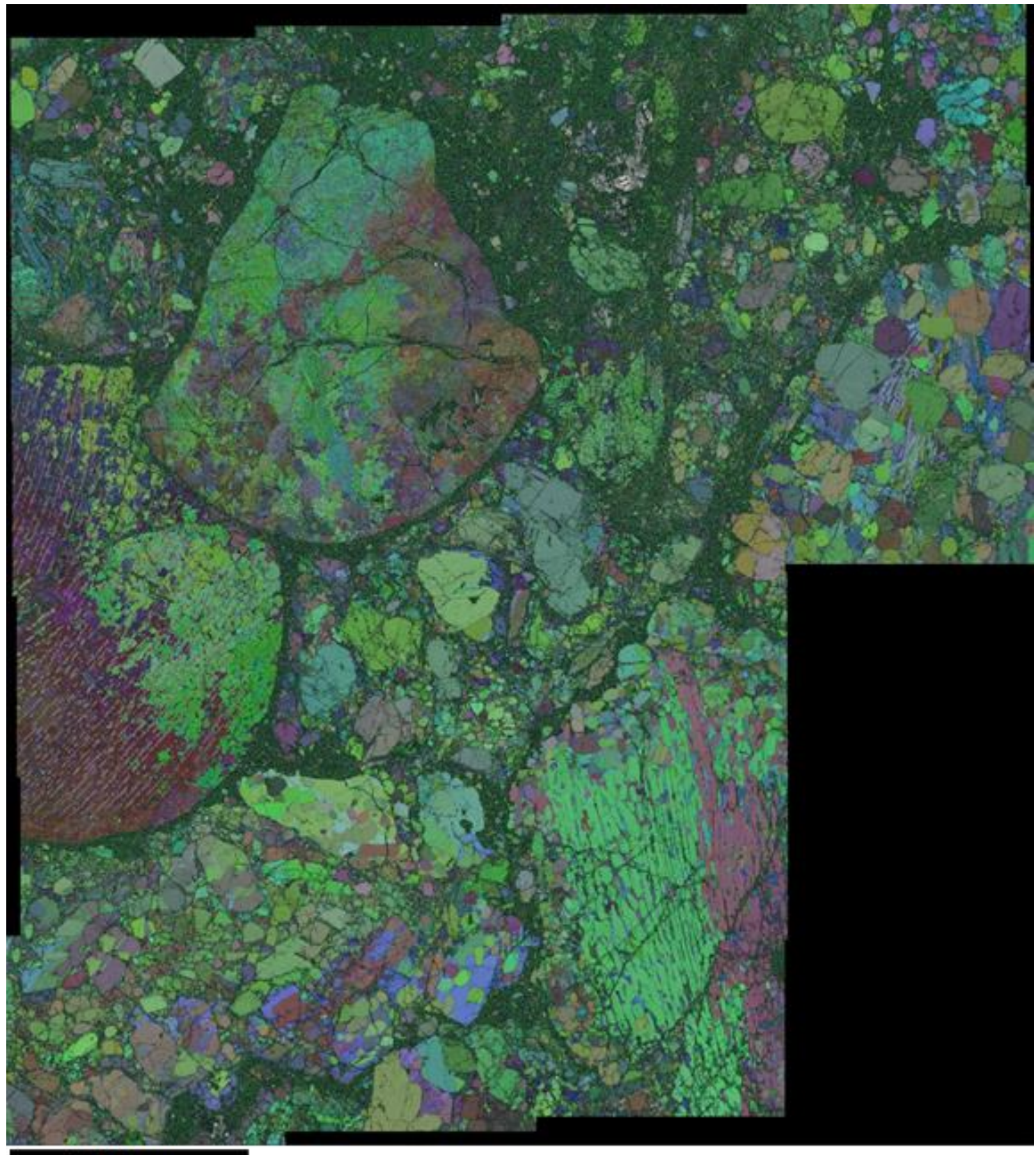

$1000 \mu \mathrm{m}$

Figure 33. Euler Map 1215 of NWA 5421. Map shows orientation of all crystal grains of all phases in a Euler coordinate system, as indicated by the color of the grains. 


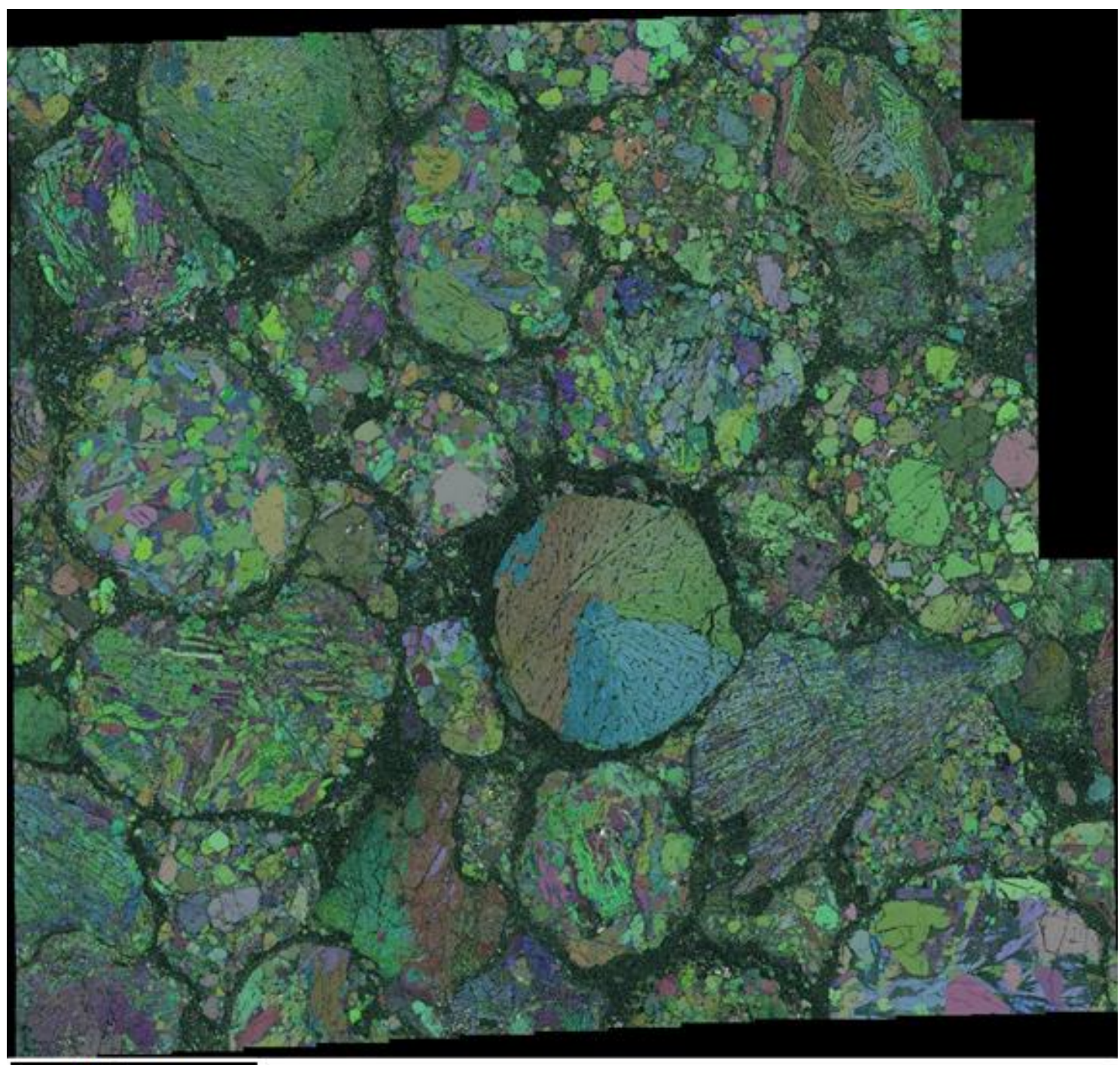

$2000 \mu \mathrm{m}$

Figure 34. Euler Map 1222 of NWA 5421. Map shows orientation of all crystal grains of all phases in a Euler coordinate system, as indicated by the color of the grains. 


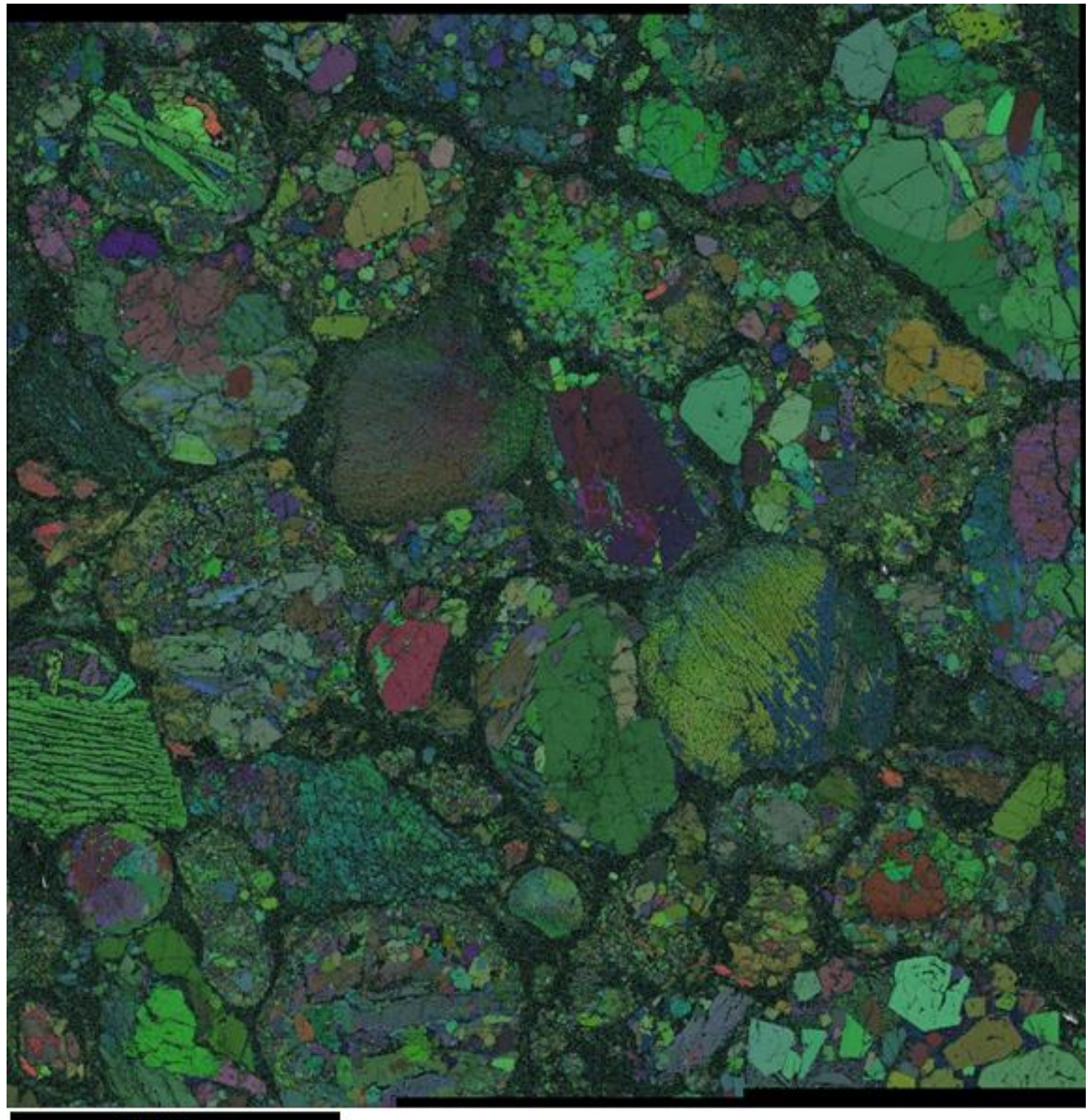

$1000 \mu \mathrm{m}$

Figure 35. Euler Map 0923 of NWA 5781. Map shows orientation of all crystal grains of all phases in a Euler coordinate system, as indicated by the color of the grains. 


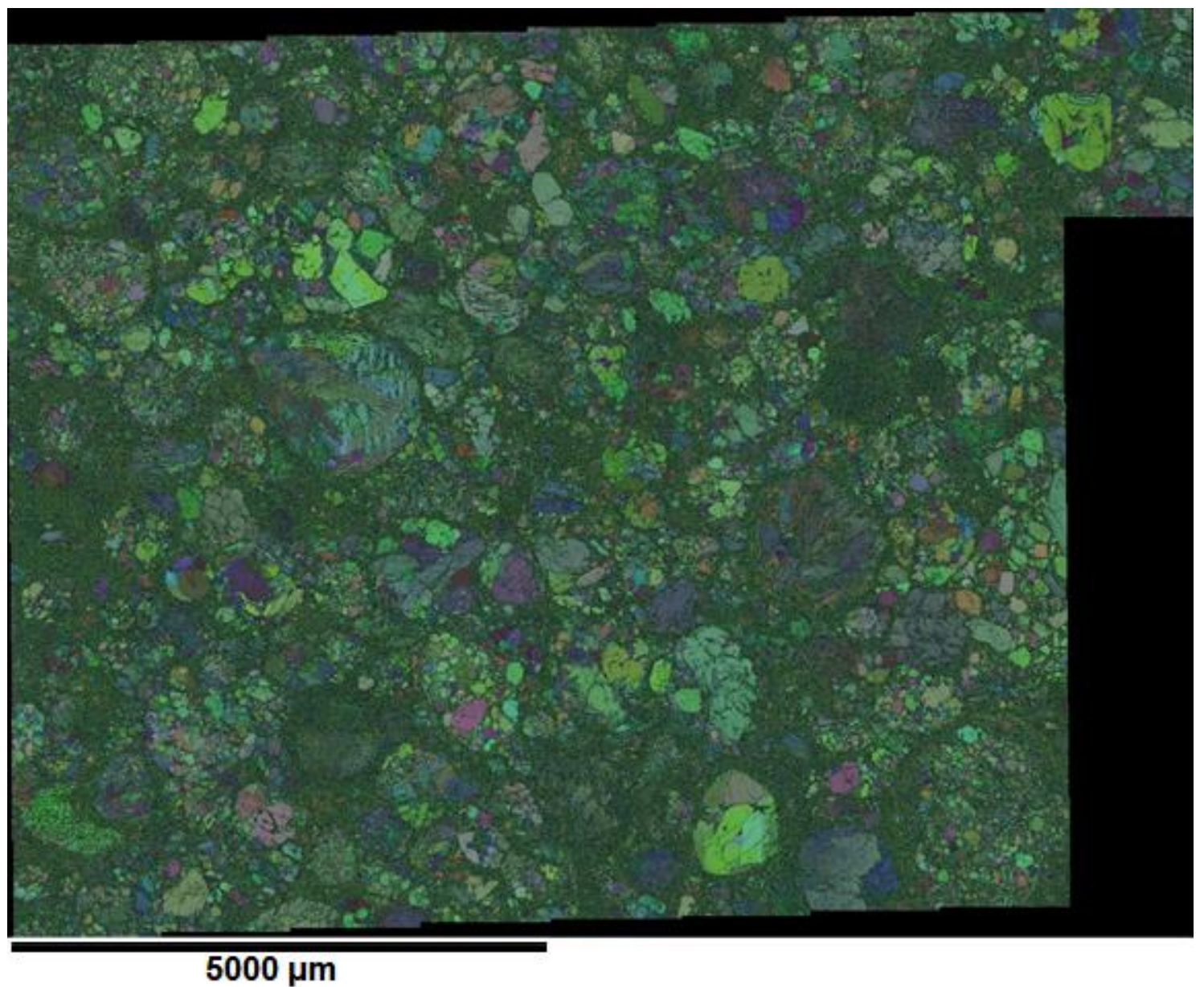

Figure 36. Euler Map 1005 of NWA 5781. Map shows orientation of all crystal grains of all phases in a Euler coordinate system, as indicated by the color of the grains. 


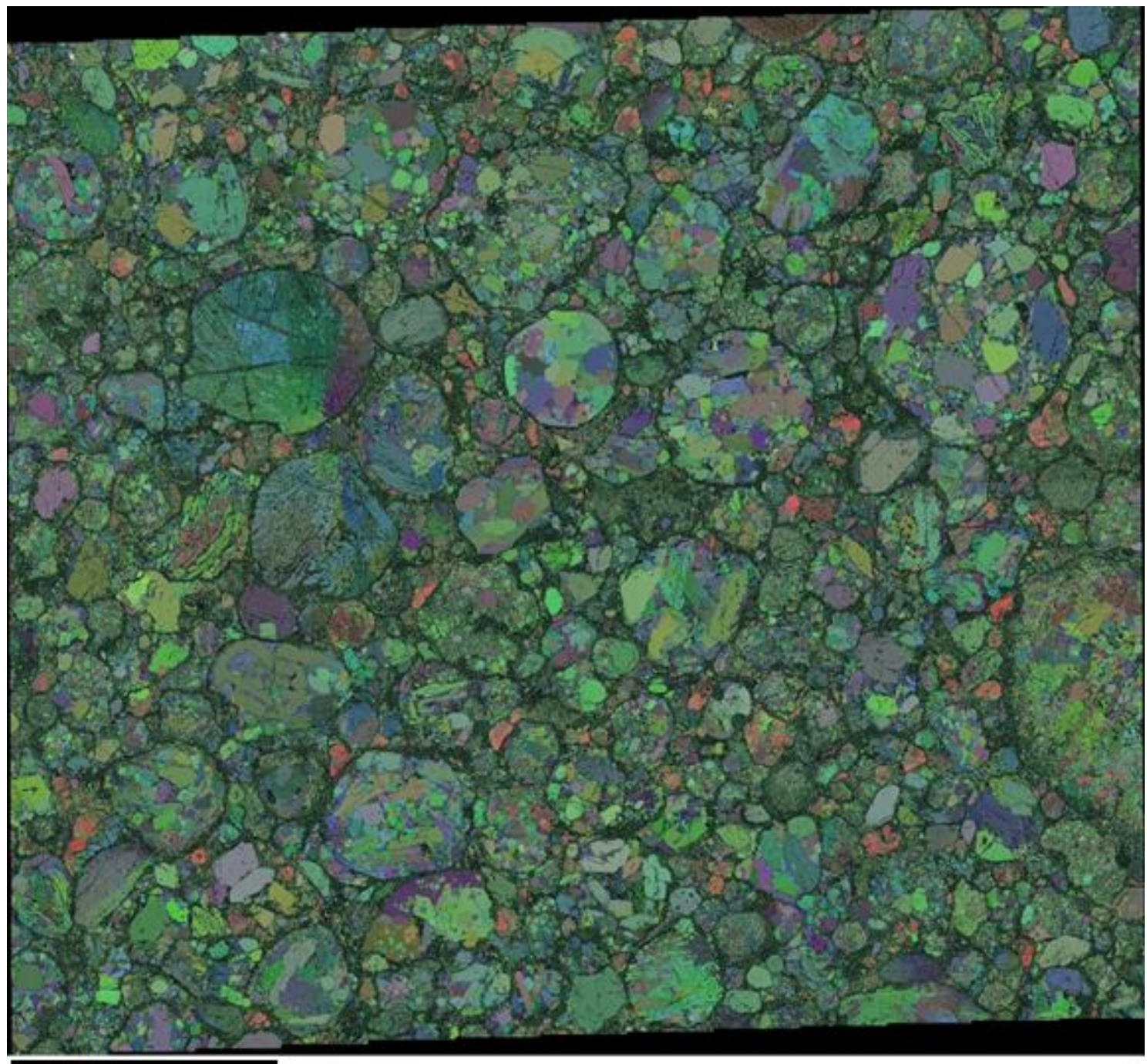

$2000 \mu \mathrm{m}$

Figure 37. Euler Map 0208 of Tieschitz. Map shows orientation of all crystal grains of all phases in a Euler coordinate system, as indicated by the color of the grains. 


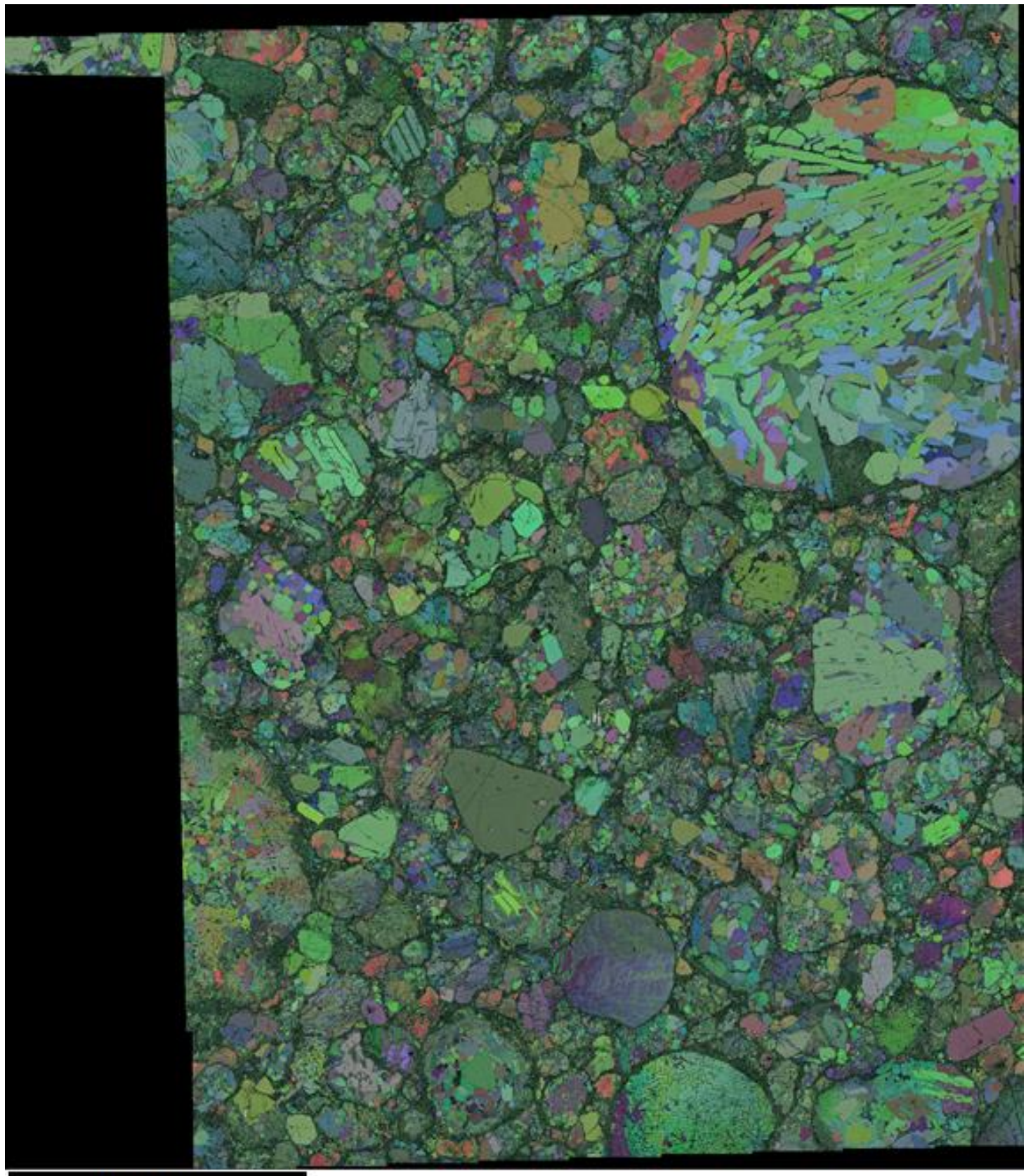

$2000 \mu \mathrm{m}$

Figure 38. Euler Map 0215 of Tieschitz. Map shows orientation of all crystal grains of all phases in a Euler coordinate system, as indicated by the color of the grains. 

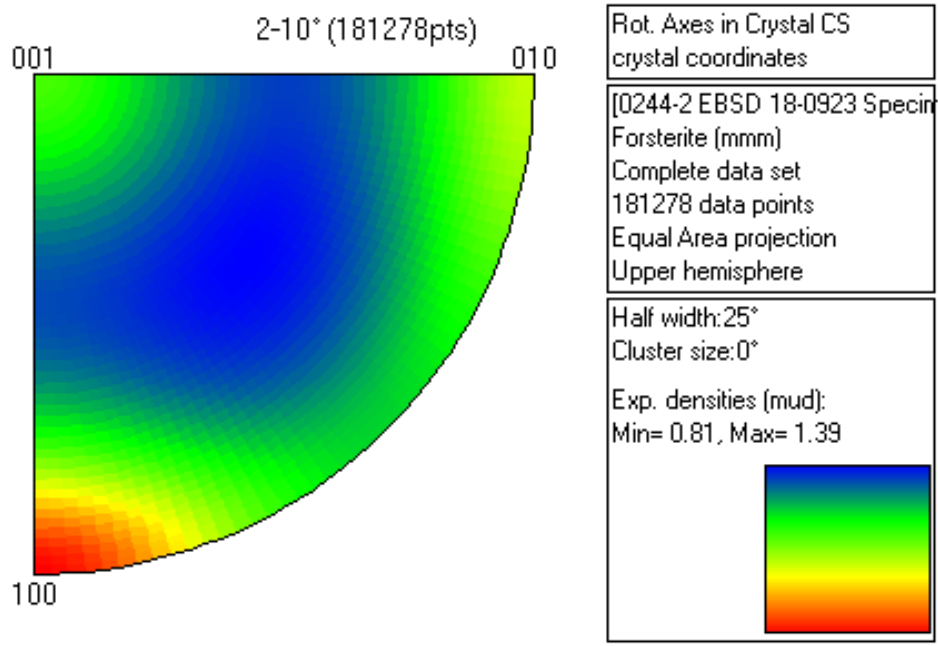

Figure 39. CRA Diagrams of EBSD Mapped Areas of NWA 5781. Upper diagram is for map 0923 (SAM), and lower diagram is for map 1005 (LAM). Hot colors indicate high density of CRA poles and cold colors indicate low density. Evidence for activation of all olivine slip systems is present.

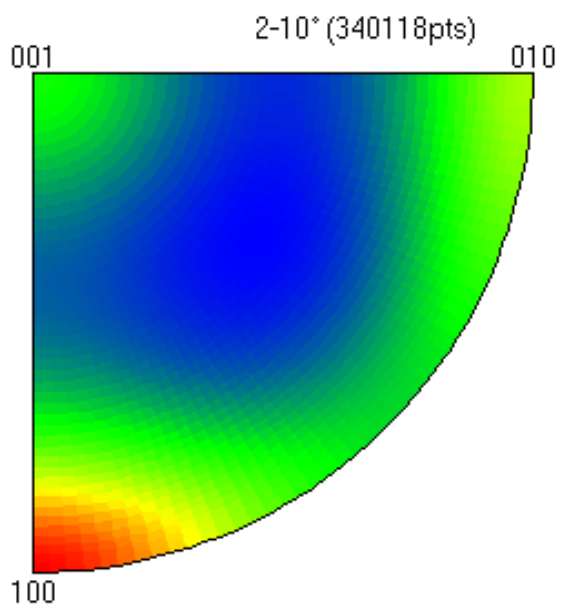

Rot. Axes in Crystal CS crystal coordinates

[0244-2 EBSD 18-1005 (LAM)

Forsterite (mmm)

Complete data set

340118 data points

Equal Area projection

Upper hemisphere

Half width: $25^{\circ}$

Cluster size: $0^{\circ}$

Exp. densities (mud):

Min $=0.80, \operatorname{Max}=1.43$

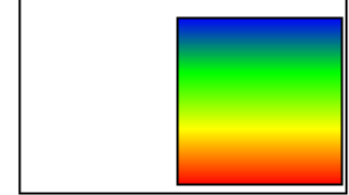
is the most activated system. 


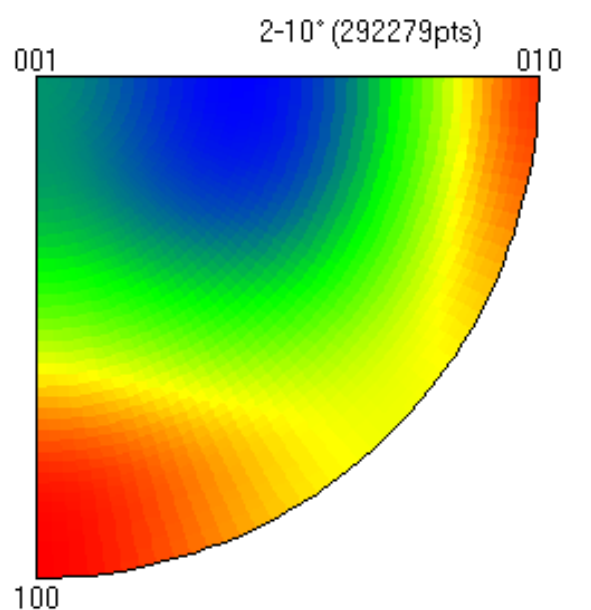

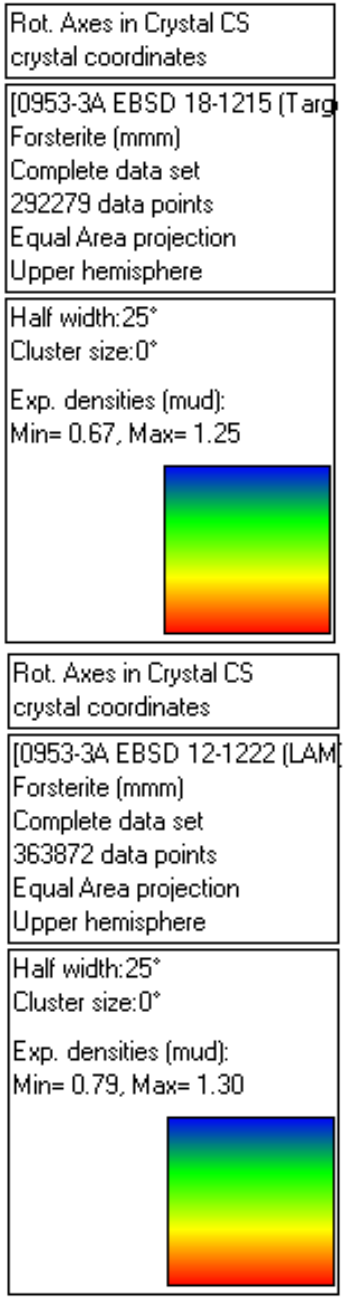

Figure 40. CRA Diagrams of EBSD Mapped Areas of NWA 5421. Upper diagram is for map 1215 (SAM), and lower diagram is for map 1222 (LAM). Hot colors indicate high density of CRA poles and cold colors indicate low density. Evidence for activation of all olivine slip systems is present.

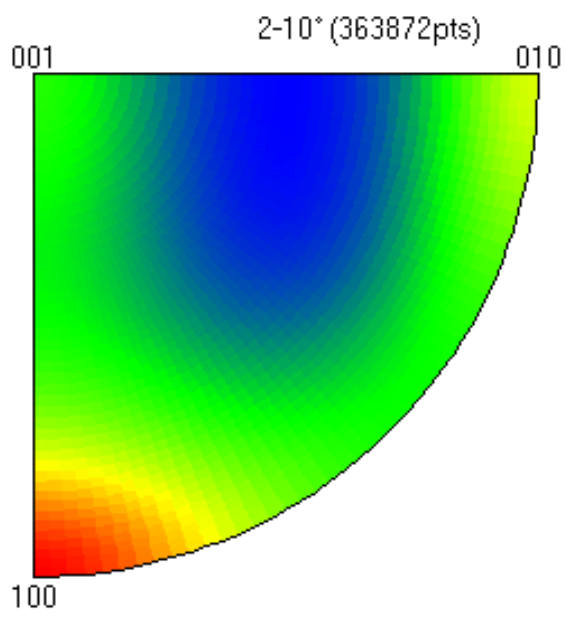
Based on Figure 4, c-slip is the most activated system. 

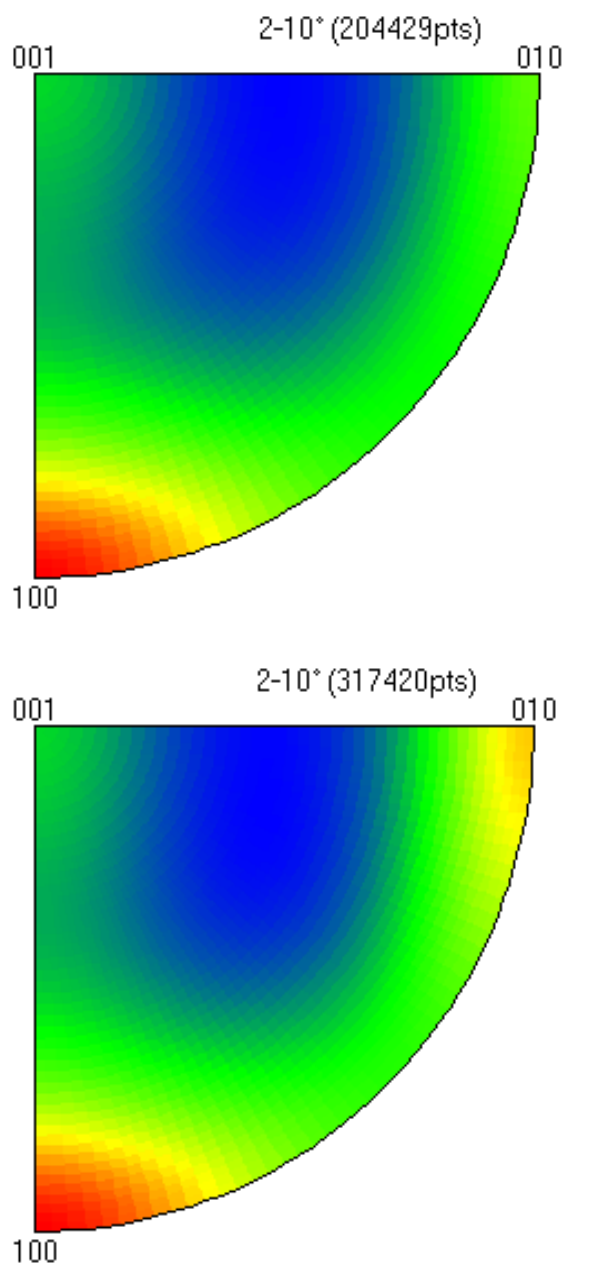

Rot. Axes in Crystal CS

crystal coordinates

[0954-1A-1 EBSD 19-0118 (mir

Forsterite (mmm)

Complete data set

204429 data points

Equal Area projection

Upper hemisphere

Half width: 25

Cluster size: $0^{\circ}$

Exp. densities (mud):

Min= 0.79, $\operatorname{Max}=1.39$

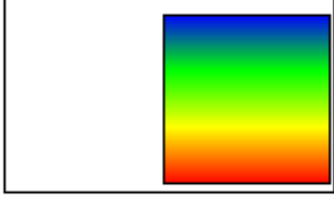

Rot. Axes in Crystal CS

crystal coordinates

[0954-1A-1 EBSD 19-0201 (LA Forsterite (mmm)

Clast

317420 data points

Equal Area projection

Upper hemisphere

Half width: $25^{\circ}$

Cluster size: $0^{\circ}$

Exp. densities (mud):

Min $=0.81, \operatorname{Max}=1.29$
Figure 41. CRA

Diagrams of EBSD

Mapped Areas of NWA 5205. Upper diagram is for map 0118 (SAM), and lower diagram is for map 0201 (LAM). Hot colors indicate high density of CRA poles and cold colors indicate low density. Evidence for activation of all olivine slip systems is present. Based on Figure 4, c-slip is the most activated system. 

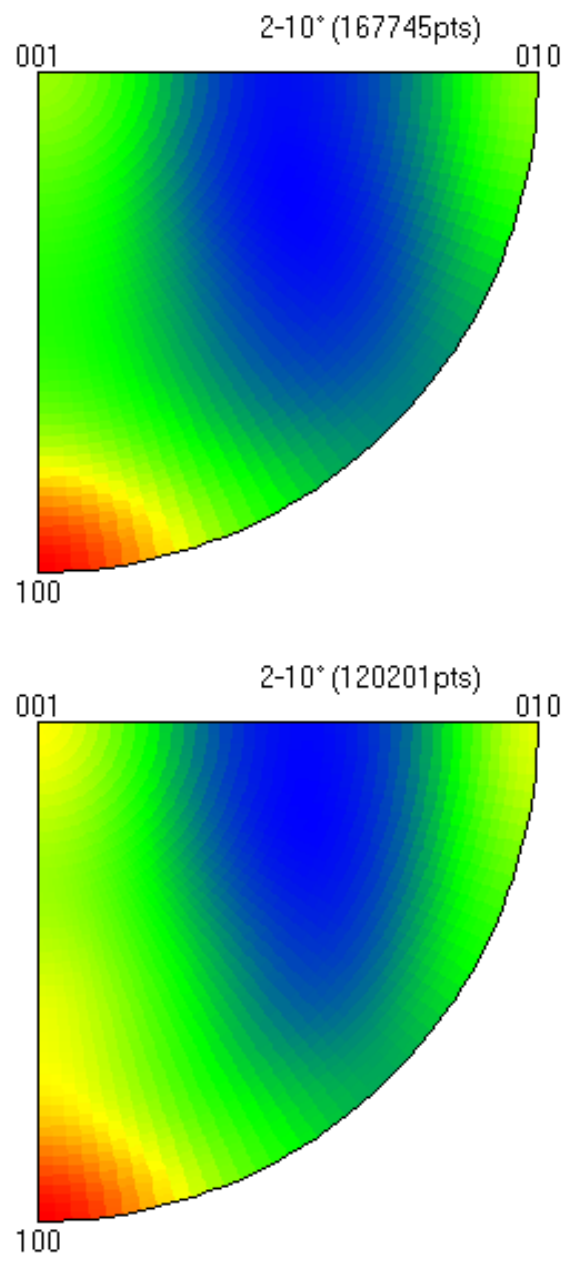

Rot. Axes in Crystal CS

crystal coordinates

[0781-B EBSD 19-0208 (LAM)

Forsterite (mmm)

Complete data set

167745 data points

Equal Area projection

Upper hemisphere

Half width: $25^{\circ}$

Cluster size: $0^{\circ}$

Exp. densities (mud):

$\mathrm{Min}=0.83, \mathrm{Max}=1.29$

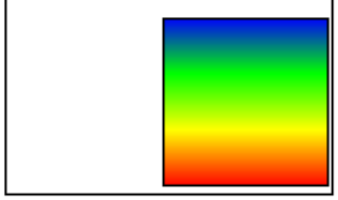

Rot. Axes in Crystal CS

crystal coordinates

[0781-B EBSD 19-0215 (LAM2

Forsterite (mmm)

Non-0verlap with LAM 1 Area

120201 data points

Equal Area projection

Upper hemisphere

Half width:25

Cluster size: $0^{\circ}$

Exp. densities (mud):

Min=0.83, $\operatorname{Max}=1.19$
Figure 42.

CRA

Diagrams of EBSD

Mapped Areas of

Tieschitz. Upper

diagram is for map 0208 (SAM), and lower diagram is for map 0215 (LAM). Hot colors indicate high density of CRA poles and cold colors indicate low density. Evidence for activation of all olivine slip systems is present. Based on Figure 4, c-slip is the most activated system. 

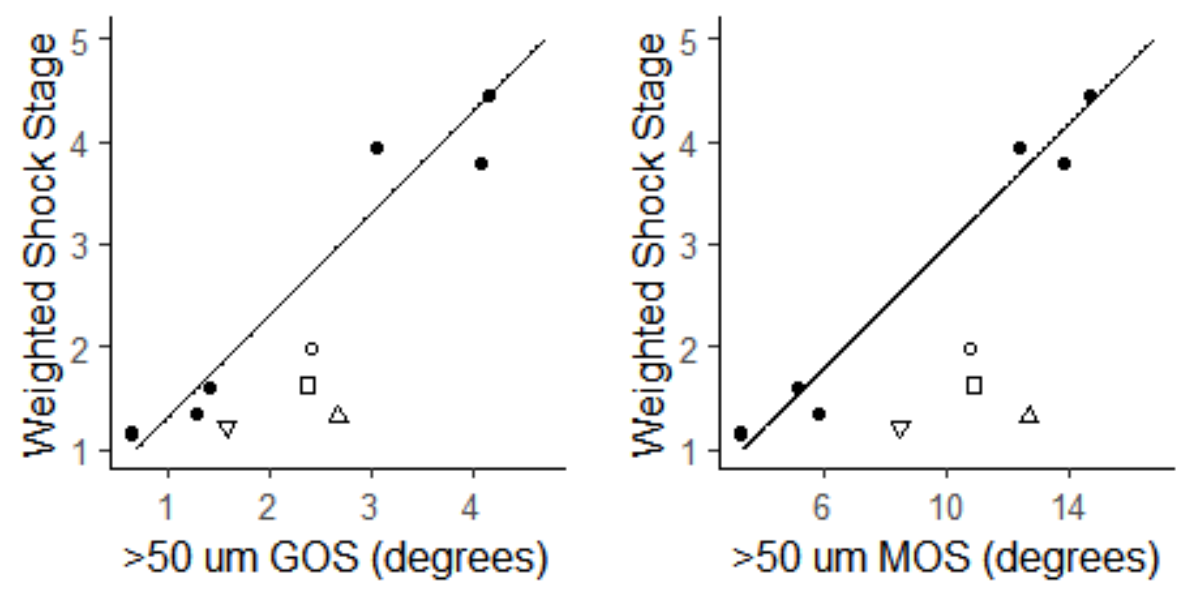

Legend

- NWA 5205

$\triangle$ NWA 5421

- NWA 5781

$\nabla$ Tieschtiz

- RH2018

- Trendline

Figure 43. Diagrams Comparing GOS and MOS to Weighted Shock Stages. GOS values are calculated from the combined olivine grain populations of map datasets. Data of this study depart from the trend established in Ruzicka and Hugo (2018), but are in regions of GOS, MOS, and weighted shock stages outside those of that study. In the legend, "RH2018" is an abbreviation representing data from Ruzicka and Hugo (2018). 


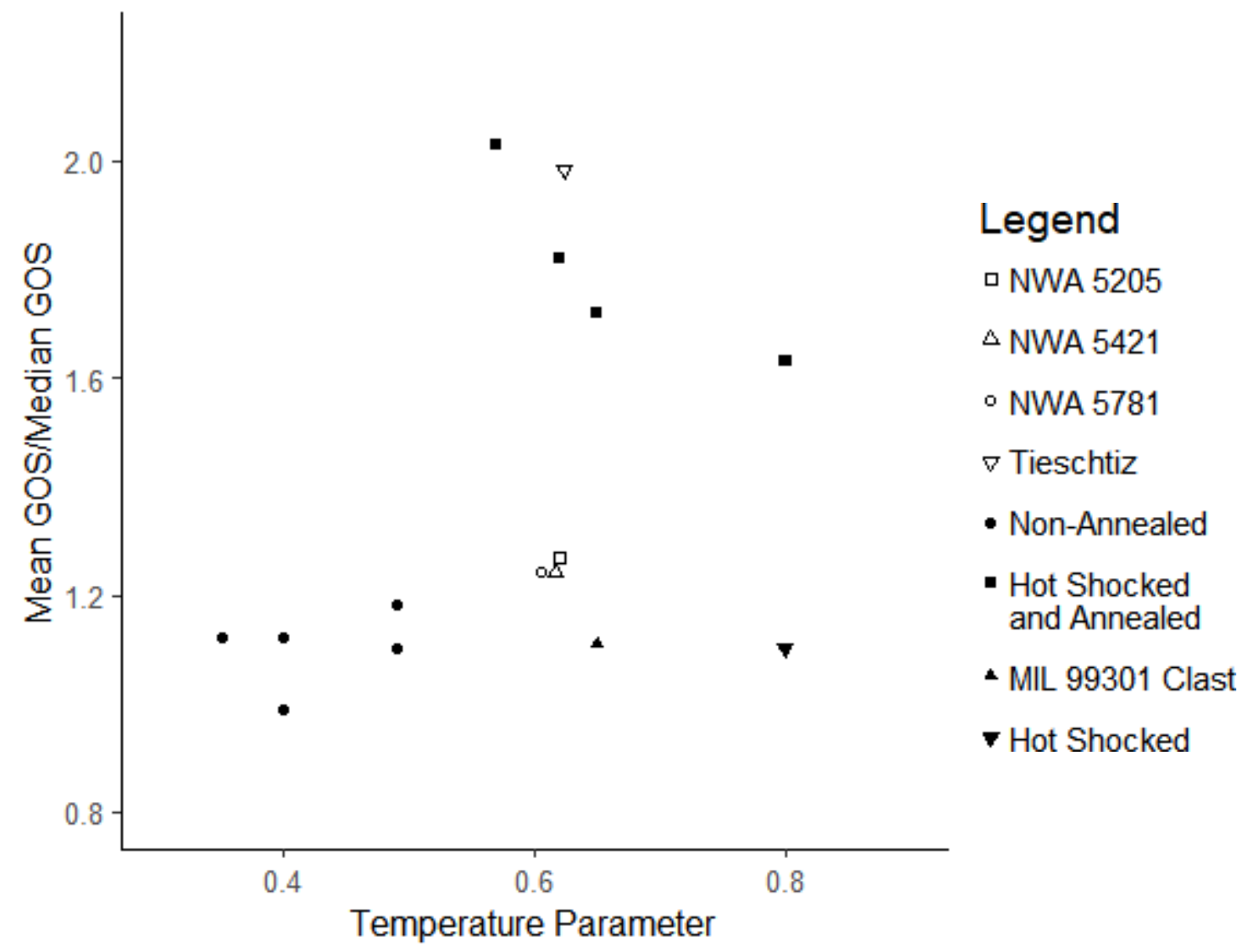

Figure 44. Graph Comparing Mean GOS/Median GOS and Temperature Parameters. Black points represent data from Ruzicka and Hugo (2018) and Hugo et al. (2019) shocked samples, white points are data from this study. The clast in MIL 99301 was interpreted as a shock melt that was deformed and incorporated into the host. "Hot shocked" refers to Saint-Séverin, which was interpreted as being shocked while hot due to thermal metamorphism and has had little annealing (Hugo et al., 2019). "Hot shocked and annealed" refers to samples that were shocked while hot due to thermal metamorphism and subsequently annealed. "Non-Annealed" refers to samples shocked while cold. Plotted points for this study's samples represent the combined datasets of both maps for each sample. Samples of this study all have approximately $60 \%$ a-slip activation. Most samples analyzed in this study form a tight cluster distinct from the clusters of Ruzicka and Hugo (2018) and Hugo et al. (2019). Tieschitz plots with the annealed samples, but unlike them, is unequilibrated. 


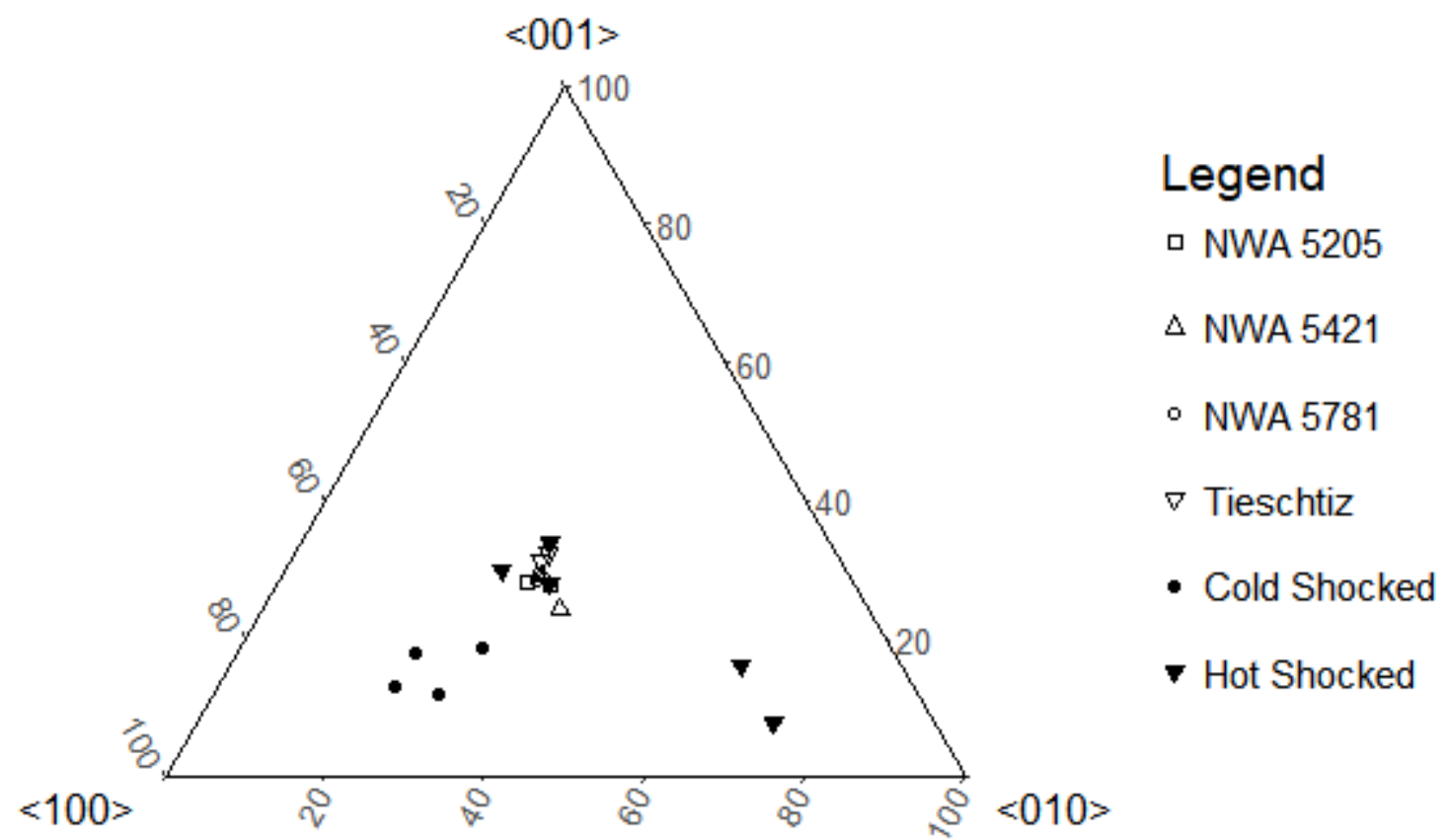

Figure 45. Ternary Displaying Modal Crystal Rotation Axes in Analyzed Samples. Samples are highly similar with subequal activation of cold $(<100\rangle$ CRAs), intermediately hot $(<010>$ CRAs) and very hot $(<001>$ CRAs). One point is plotted per EBSD mapped area. Shocked samples are from Ruzicka and Hugo (2018) and Hugo et al. (2019). Cold shocked samples were shock metamorphosed after thermal metamorphism. Hot shocked samples were shock metamorphosed during thermal metamorphism and include both annealed and unannealed meteorites.

\subsection{Chondrule Results}

Data for regional subsets are shown in Table 7. Tables 8-11 show chondrule subset data for the samples. Figures 46-53 identify analyzed chondrules in the EBSD maps and display localization of the chondrule data. Most individual chondrules have temperature parameters consistent with deformation under elevated temperatures, with only 2 of 80 chondrules having temperature parameters consistent with cold deformation. See Figure 54. Most chondrule modal slip systems are roughly consistent with their associated whole maps, however, Tieschitz is exceptionally variable, see Figures 55-58. It is also found that there is no significant correlation between the temperature parameters and shape parameters or mean GOS values of chondrules, however, there is a suggestive relationship 
between the mean GOS values and shape parameters of chondrules, though not one that is statistically significant by the set methodological criteria. See Figures 59-61.

Table 7. Regional Subset CRA Data. Columns are labeled by sample and map, rows are divided by subset and show MUD values and $\left.f_{\langle 010\rangle}+f<001\right\rangle$ values which are labeled as "T Param." $f<010\rangle+\mathbf{f}<001>$ is the sum of the MUD values of the two aslip systems in CRA stereonets; it is synonymous with the temperature parameter. $f_{<100>}$ is the MUD value of CRA poles associated with c-slip. Map designations are the same as those in Tables 4 and 5.

\begin{tabular}{|c|c|c|c|c|c|c|c|c|}
\hline & \multicolumn{2}{|c|}{ NWA 5205} & \multicolumn{2}{|c|}{ NWA 5421} & \multicolumn{2}{|c|}{ NWA 5781} & \multicolumn{2}{|c|}{ Tieschitz } \\
\hline & 0118 & 0201 & 1215 & 1222 & 0923 & 1005 & 0208 & 0215 \\
\hline \multicolumn{9}{|l|}{ Subset 1} \\
\hline $\mathrm{f}_{\langle 100\rangle}$ & 1.27 & 1.19 & 1.17 & 1.28 & 1.55 & 1.40 & 1.28 & 1.21 \\
\hline $\mathrm{f}_{\langle 010\rangle}$ & 1.20 & 1.33 & 1.17 & 1.06 & 0.99 & 1.15 & 1.01 & 0.99 \\
\hline $\mathrm{f}_{\langle 001\rangle}$ & 0.98 & 0.92 & 0.97 & 1.02 & 1.01 & 1.03 & 1.14 & 1.08 \\
\hline T Param. & 0.632 & 0.654 & 0.647 & 0.619 & 0.563 & 0.609 & 0.627 & 0.631 \\
\hline \multicolumn{9}{|l|}{ Subset 2} \\
\hline $\mathrm{f}_{\langle 100\rangle}$ & 1.35 & 1.20 & 1.33 & 1.26 & 1.25 & 1.47 & 1.30 & 1.25 \\
\hline $\mathrm{f}_{\langle 010\rangle}$ & 1.06 & 1.07 & 1.18 & 1.14 & 1.32 & 1.13 & 1.10 & 0.97 \\
\hline$f_{\langle 001\rangle}$ & 1.01 & 1.03 & 0.60 & 0.98 & 1.00 & 1.00 & 1.03 & 1.14 \\
\hline T Param. & 0.605 & 0.636 & 0.572 & 0.627 & 0.650 & 0.592 & 0.621 & 0.628 \\
\hline \multicolumn{9}{|l|}{ Subset 3} \\
\hline $\mathrm{f}_{\langle 100\rangle}$ & 1.25 & 1.25 & 1.17 & 1.34 & 1.51 & 1.56 & 1.26 & 1.14 \\
\hline $\mathrm{f}_{\langle 010\rangle}$ & 1.10 & 1.13 & 1.23 & 1.12 & 1.01 & 1.13 & 1.12 & 1.06 \\
\hline$f_{\langle 001\rangle}$ & 1.01 & 0.93 & 0.75 & 0.99 & 1.14 & 0.98 & 1.02 & 1.01 \\
\hline T Param. & 0.628 & 0.622 & 0.629 & 0.612 & 0.587 & 0.575 & 0.629 & 0.645 \\
\hline \multicolumn{9}{|l|}{ Subset 4} \\
\hline $\mathrm{f}_{\langle 100\rangle}$ & 1.36 & 1.31 & 1.25 & 1.32 & 1.22 & 1.39 & 1.24 & 1.17 \\
\hline $\mathrm{f}_{\langle 010\rangle}$ & 1.08 & 1.18 & 1.25 & 1.14 & 1.28 & 1.16 & 1.08 & 1.13 \\
\hline $\mathrm{f}_{\langle 001\rangle}$ & 0.94 & 0.94 & 0.83 & 0.96 & 1.07 & 1.04 & 1.09 & 1.07 \\
\hline T Param. & 0.598 & 0.618 & 0.625 & 0.614 & 0.658 & 0.613 & 0.636 & 0.653 \\
\hline \multicolumn{9}{|l|}{ Subset 5} \\
\hline $\mathrm{f}_{\langle 100\rangle}$ & 1.71 & 1.38 & 1.23 & 1.32 & 1.40 & 1.29 & 1.34 & 1.19 \\
\hline $\mathrm{f}_{\langle 010\rangle}$ & 0.92 & 1.14 & 1.16 & 1.05 & 1.17 & 1.18 & 1.04 & 1.07 \\
\hline $\mathrm{f}_{\langle 001\rangle}$ & 0.86 & 0.91 & 1.07 & 0.99 & 1.07 & 1.09 & 1.07 & 1.08 \\
\hline T Param. & 0.510 & 0.598 & 0.645 & 0.607 & 0.615 & 0.638 & 0.612 & 0.644 \\
\hline
\end{tabular}


Table 8. Chondrule Data Table for NWA 5205. Map designations are the same as those in Tables 4 and 5 . Chondrules intersecting the borders of maps do not have recorded shape parameters or mean GOS values, though MUD data is available for all chondrules. $f_{<010\rangle}+f_{<001>}$ is the sum of the MUD values of the two a-slip systems in CRA stereonets; it is synonymous with the temperature parameter. Map 201 only has data derived from the sample's cluster chondrite clast; host material was digitally removed.

\begin{tabular}{lcccc}
\hline Map & Chondrule & Shape Parameter & Mean GOS & $\mathrm{f}_{<010>}+\mathrm{f}_{<001>}$ \\
\hline 0118 & 1 & 1.06 & 0.69 & 0.560 \\
0118 & 2 & - & - & 0.604 \\
0118 & 3 & - & - & 0.414 \\
0118 & 4 & 1.16 & 2.96 & 0.630 \\
0118 & 5 & 1.11 & 2.15 & 0.630 \\
0118 & 6 & - & - & 0.597 \\
0118 & 7 & - & - & 0.608 \\
0118 & 8 & - & - & 0.541 \\
0118 & 9 & - & - & 0.654 \\
0118 & 10 & - & - & 0.565 \\
0201 & 1 & 1.34 & 2.47 & 0.680 \\
0201 & 2 & 1.23 & 2.76 & 0.664 \\
0201 & 3 & 1.08 & 2.09 & 0.630 \\
0201 & 4 & 1.40 & 2.29 & 0.671 \\
0201 & 5 & 1.08 & 2.48 & 0.519 \\
0201 & 6 & 1.19 & 2.75 & 0.645 \\
0201 & 7 & 1.09 & 2.37 & 0.649 \\
0201 & 8 & 1.58 & 2.69 & 0.636 \\
0201 & 9 & 1.17 & 2.95 & 0.594 \\
0201 & 10 & 1.33 & 2.50 & 0.632 \\
\hline
\end{tabular}


Table 9. Chondrule Data Table for NWA 5421. Map designations are the same as those in Tables 4 and 5. Chondrules intersecting the borders of maps do not have recorded shape parameters or mean GOS values, though MUD data is available for all chondrules. $f_{<010\rangle}+f_{<001}>$ is the sum of the MUD values of the two a-slip systems in CRA stereonets; it is synonymous with the temperature parameter.

\begin{tabular}{lcccc}
\hline Map & Chondrule & Shape Parameter & Mean GOS & $\mathrm{f}_{<010>}+\mathrm{f}_{<001>}$ \\
\hline 1215 & 1 & - & - & 0.642 \\
1215 & 2 & - & - & 0.586 \\
1215 & 3 & 1.38 & 2.59 & 0.645 \\
1215 & 4 & - & - & 0.665 \\
1215 & 5 & - & - & 0.631 \\
1215 & 6 & 1.11 & 2.56 & 0.541 \\
1215 & 7 & - & - & 0.665 \\
1215 & 8 & - & - & 0.706 \\
1215 & 9 & - & - & 0.621 \\
1215 & 10 & 1.15 & 1.94 & 0.575 \\
1222 & 1 & 1.18 & 2.95 & 0.542 \\
1222 & 2 & 1.45 & 2.97 & 0.676 \\
1222 & 3 & 1.19 & 2.96 & 0.611 \\
1222 & 4 & 1.29 & 2.16 & 0.623 \\
1222 & 5 & 1.40 & 2.71 & 0.661 \\
1222 & 6 & 1.09 & 0.91 & 0.617 \\
1222 & 7 & - & - & 0.638 \\
1222 & 8 & 1.36 & 2.66 & 0.608 \\
1222 & 9 & 1.07 & 1.29 & 0.545 \\
1222 & 10 & 1.16 & 2.69 & 0.623 \\
\hline
\end{tabular}


Table 10. Chondrule Data Table for NWA 5781. Map designations are the same as those in Tables 4 and 5 . Chondrules intersecting the borders of maps do not have recorded shape parameters or mean GOS values, though MUD data is available for all chondrules. $f_{\langle 010\rangle}+f_{<001}>$ is the sum of the MUD values of the two a-slip systems in CRA stereonets; it is synonymous with the temperature parameter.

\begin{tabular}{lcccc}
\hline Map & Chondrule & Shape Parameter & Mean GOS & $\mathrm{f}_{<010>}+\mathrm{f}_{<001>}$ \\
\hline 0923 & 1 & 1.08 & 1.29 & 0.510 \\
0923 & 2 & 1.12 & 2.36 & 0.668 \\
0923 & 3 & 1.19 & 2.07 & 0.637 \\
0923 & 4 & 1.30 & 1.71 & 0.652 \\
0923 & 5 & 1.17 & 2.36 & 0.548 \\
0923 & 6 & 1.15 & 2.84 & 0.508 \\
0923 & 7 & 1.25 & 2.48 & 0.677 \\
0923 & 8 & 1.20 & 1.81 & 0.610 \\
0923 & 9 & - & - & 0.587 \\
0923 & 10 & 1.41 & 2.64 & 0.677 \\
1005 & 1 & 1.12 & 1.41 & 0.640 \\
1005 & 2 & 1.28 & 1.59 & 0.647 \\
1005 & 3 & 1.03 & 1.57 & 0.598 \\
1005 & 4 & 1.15 & 1.64 & 0.702 \\
1005 & 5 & 1.09 & 1.15 & 0.524 \\
1005 & 6 & - & - & 0.641 \\
1005 & 7 & - & - & 0.650 \\
1005 & 8 & 1.11 & 1.09 & 0.642 \\
1005 & 9 & 1.09 & 1.40 & 0.653 \\
1005 & 10 & 1.06 & 1.54 & 0.580 \\
\hline
\end{tabular}


Table 11. Chondrule Data Table for Tieschitz. Map designations are the same as those in Tables 4 and 5. Chondrules intersecting the borders of maps do not have recorded shape parameters or mean GOS values, though MUD data is available for all chondrules. $f_{<010\rangle}+f_{<001}>$ is the sum of the MUD values of the two a-slip systems in CRA stereonets; it is synonymous with the temperature parameter.

\begin{tabular}{lcccc}
\hline Map & Chondrule & Shape Parameter & Mean GOS & $\mathrm{f}_{<010>}+\mathrm{f}_{<001>}$ \\
\hline 0208 & 1 & 1.11 & 0.79 & 0.591 \\
0208 & 2 & 1.04 & 1.06 & 0.570 \\
0208 & 3 & 1.02 & 0.36 & 0.324 \\
0208 & 4 & 1.13 & 1.45 & 0.582 \\
0208 & 5 & 1.06 & 0.73 & 0.724 \\
0208 & 6 & 1.19 & 2.59 & 0.642 \\
0208 & 7 & 1.24 & 2.30 & 0.553 \\
0208 & 8 & 1.08 & 1.24 & 0.632 \\
0208 & 9 & 1.26 & 0.95 & 0.709 \\
0208 & 10 & 1.09 & 1.34 & 0.596 \\
0215 & 1 & - & - & 0.592 \\
0215 & 2 & - & - & 0.672 \\
0215 & 3 & 1.11 & 1.33 & 0.731 \\
0215 & 4 & 1.39 & 1.57 & 0.547 \\
0215 & 5 & 1.07 & 1.11 & 0.625 \\
0215 & 6 & 1.13 & 0.68 & 0.771 \\
0215 & 7 & 1.17 & 0.68 & 0.765 \\
0215 & 8 & 1.12 & 1.74 & 0.753 \\
0215 & 9 & 1.10 & 1.20 & 0.678 \\
0215 & 10 & 1.08 & 0.69 & 0.630 \\
\hline
\end{tabular}


Chondrule ID

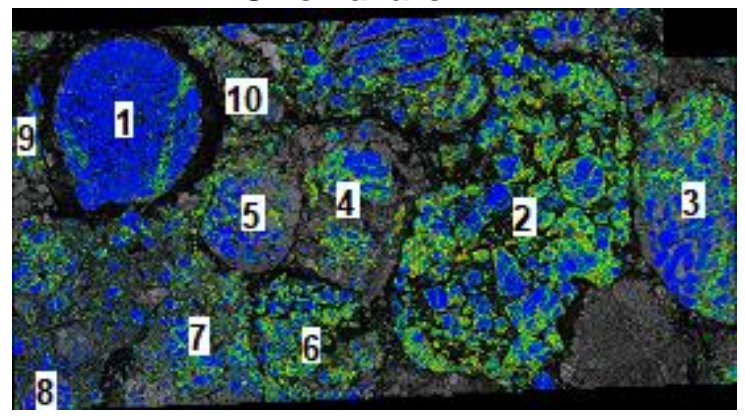

Shape Parameter

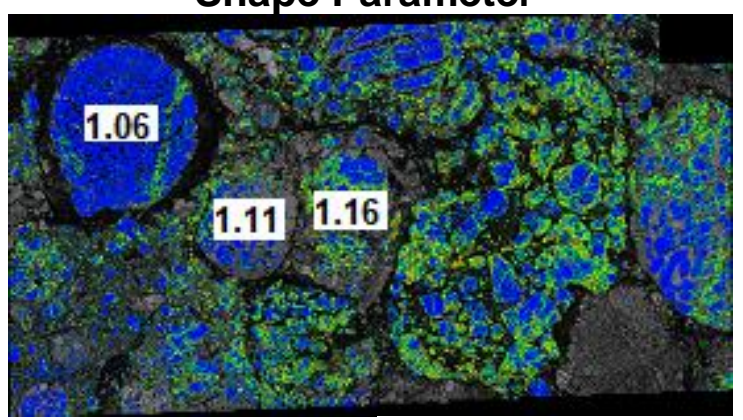

Temperature Parameter

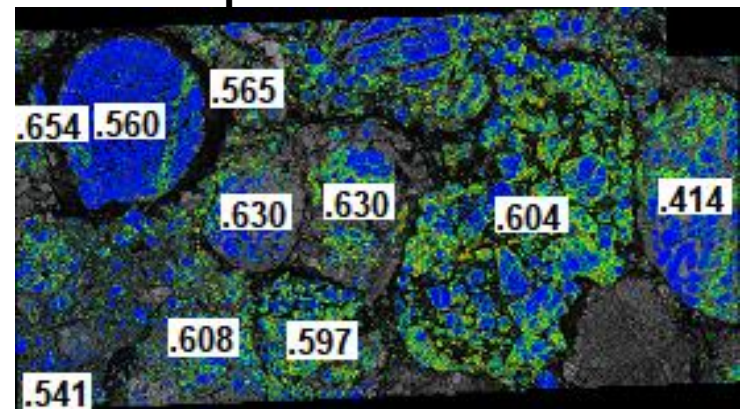

Mean GOS

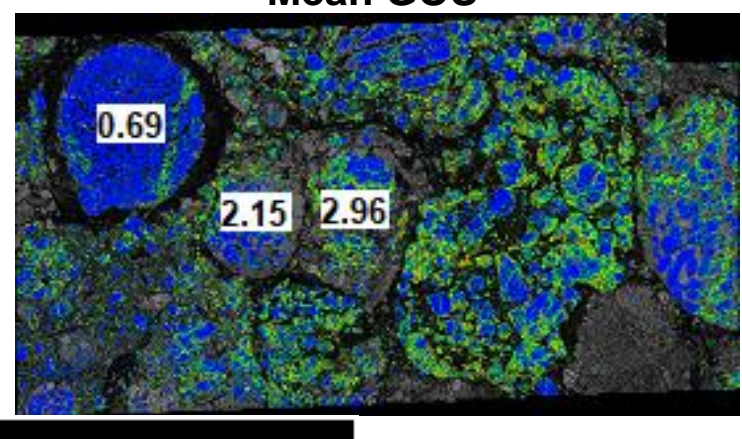

$6750 \mu \mathrm{m}$

Figure 46. Chondrule Maps of NWA 5205 Map 0118. Upper left map shows chondrule number labels. Upper right map shows $f<010\rangle+\mathbf{f}<001>$ values of the labeled chondrules. Lower left map shows shape parameters of the labeled chondrules. Lower right map shows mean GOS values $\left(^{\circ}\right)$ of the labeled chondrules. Background map is the olivine local misorientation map of map 0118. Scale applies to all maps. 

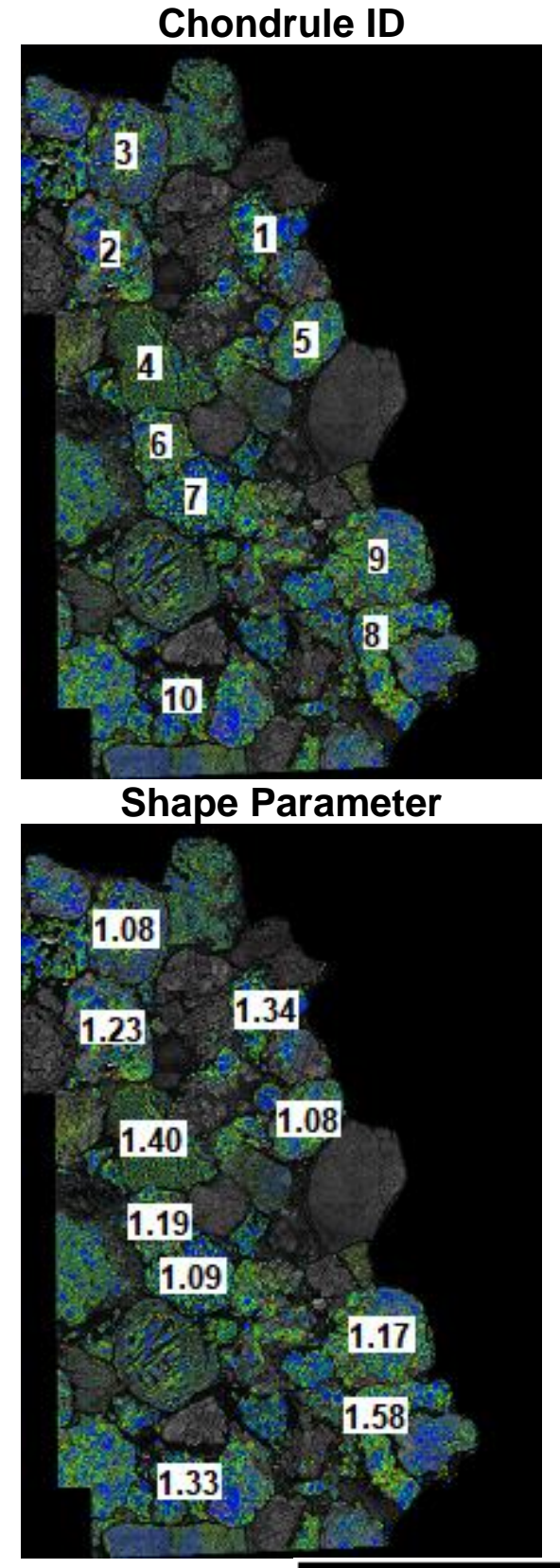

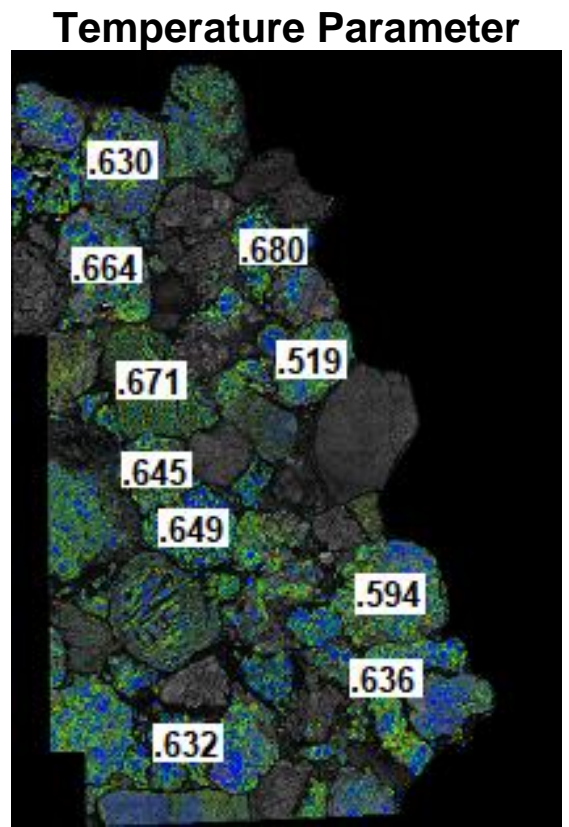

Mean GOS

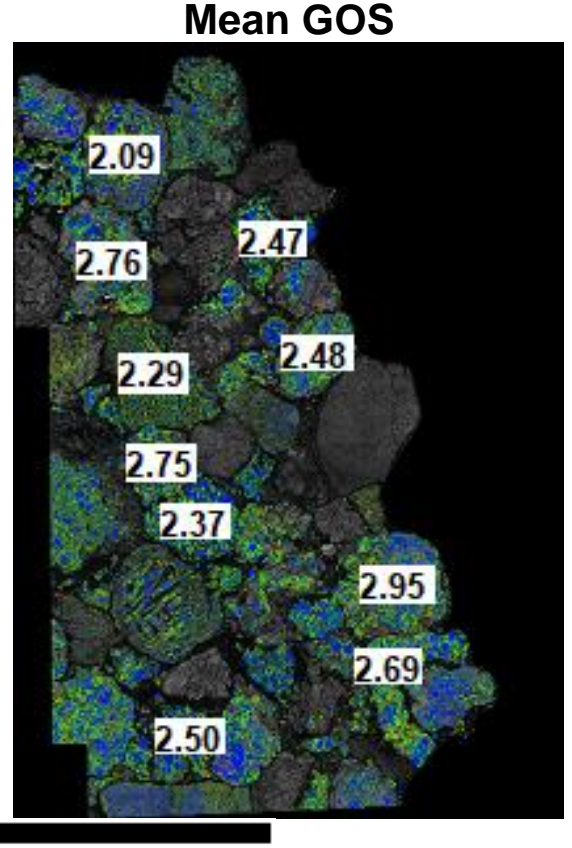

$13500 \mu \mathrm{m}$

Figure 47. Chondrule Maps of NWA 5205 Map 0201. Upper left map shows chondrule number labels. Upper right map shows $f_{\langle 010\rangle}+\mathbf{f}<001>$ values of the labeled chondrules. Lower left map shows shape parameters of the labeled chondrules. Lower right map shows mean GOS values $\left(^{\circ}\right)$ of the labeled chondrules. Background map is the olivine local misorientation map of map 0118. Scale applies to all maps. Cluster chondrite clast only; host removed digitally. 


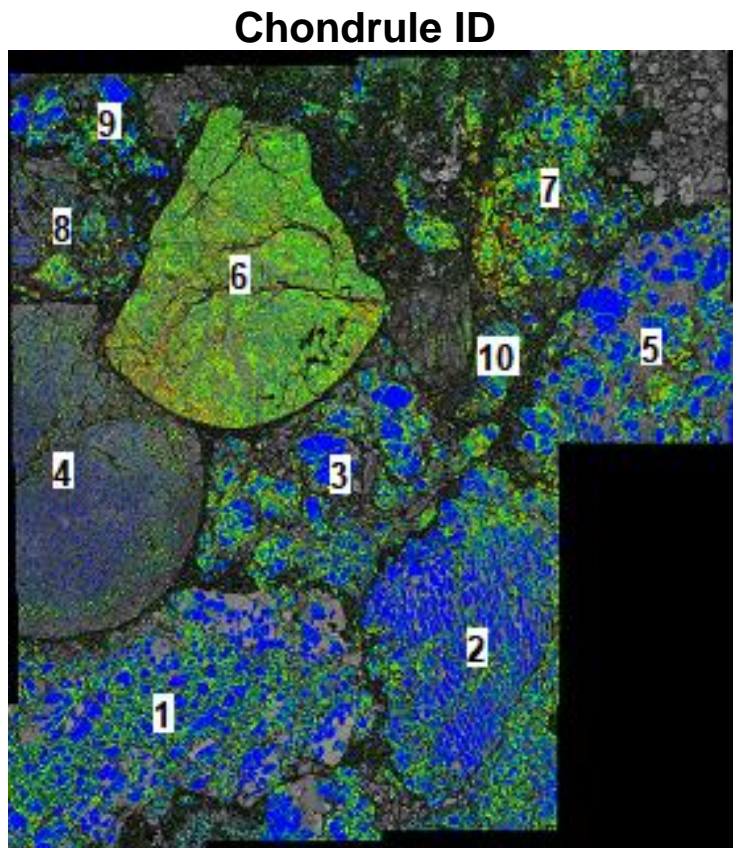

Shape Parameter

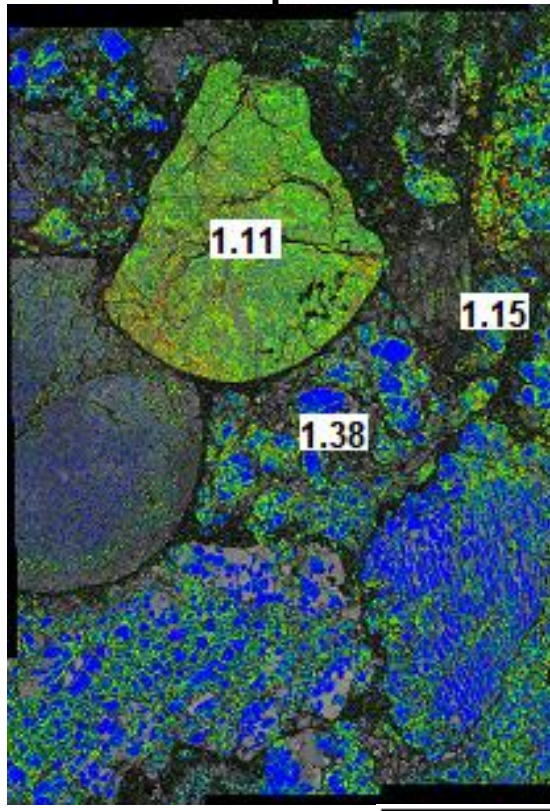

Temperature Parameter

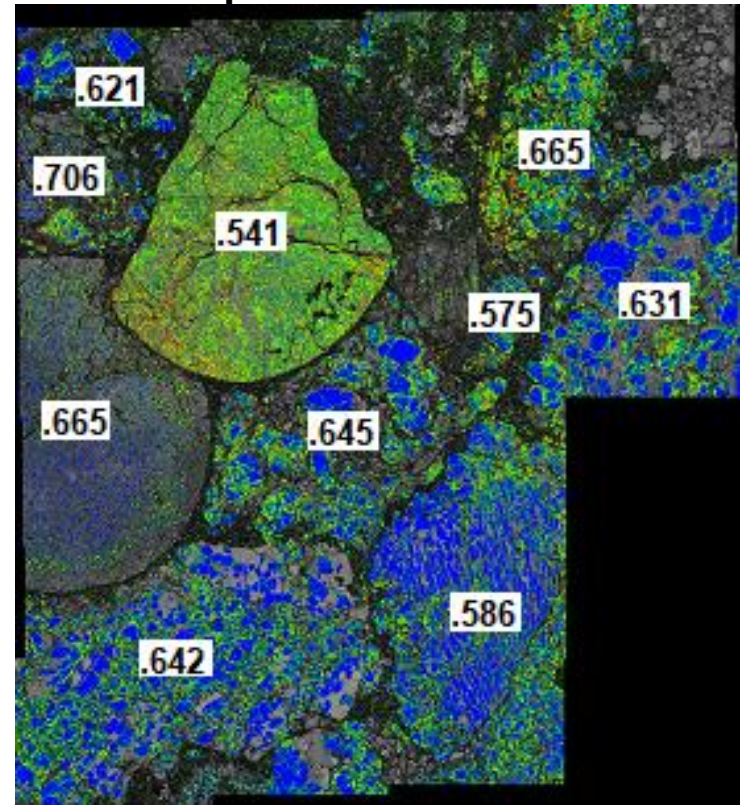

Mean GOS

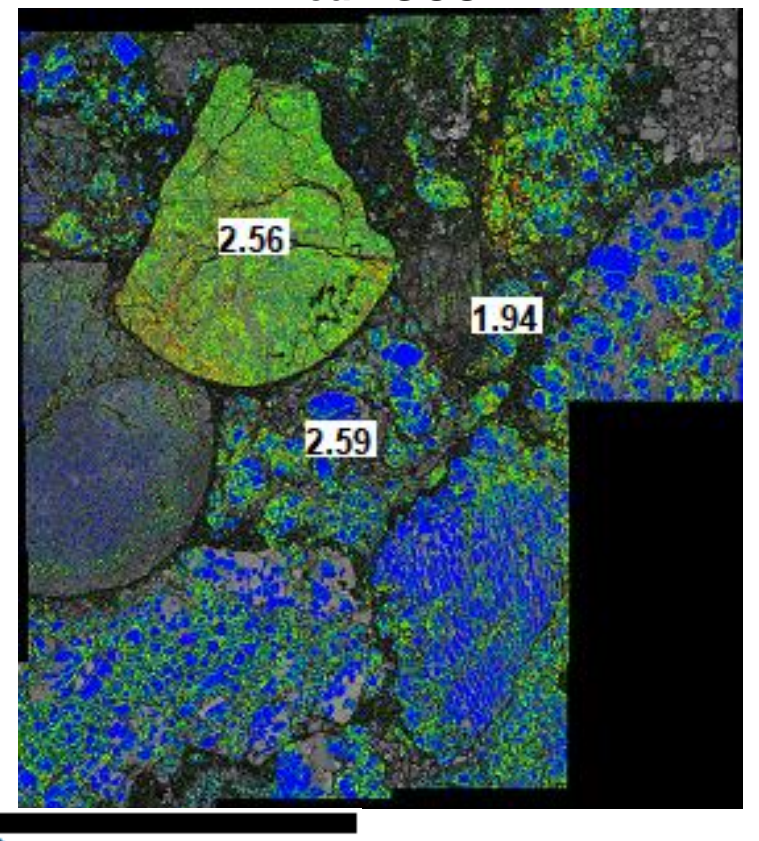

$4250 \mu \mathrm{m}$

Figure 48. Chondrule Maps of NWA 5421 Map 1215. Upper left map shows chondrule number labels. Upper right map shows $f_{\langle 010\rangle}+\mathbf{f}<001>$ values of the labeled chondrules. Lower left map shows shape parameters of the labeled chondrules. Lower right map shows mean GOS values $\left(^{\circ}\right)$ of the labeled chondrules. Background map is the olivine local misorientation map of map 1215. Scale applies to all maps. 


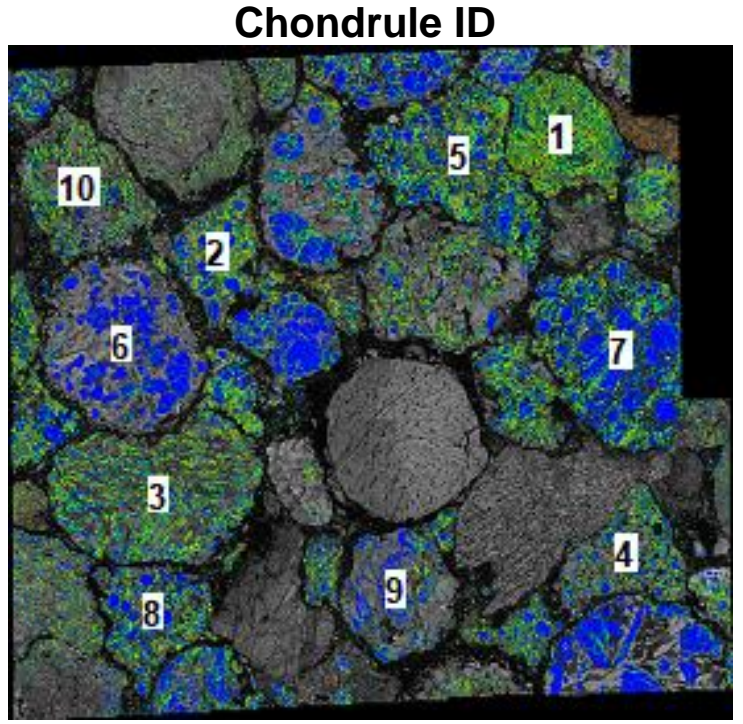

Shape Parameter

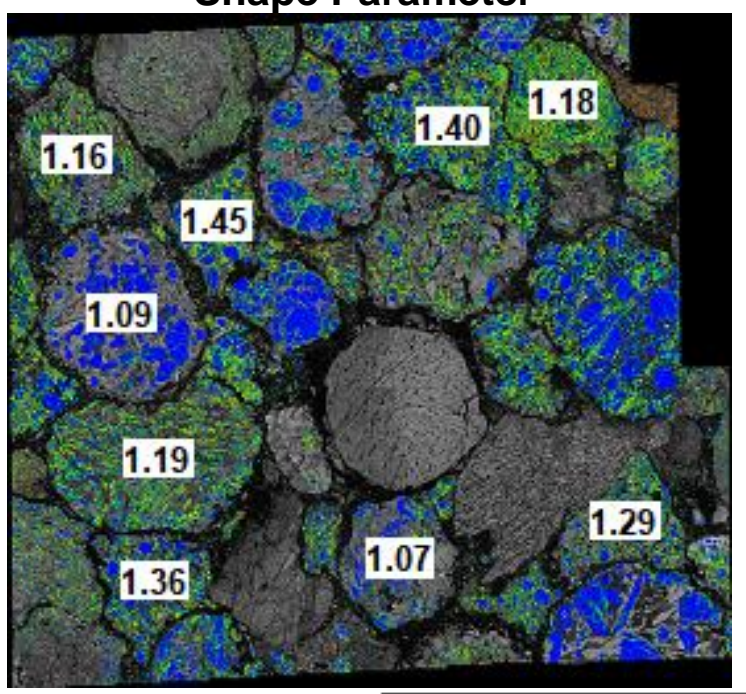

$9000 \mu \mathrm{m}$

Temperature Parameter

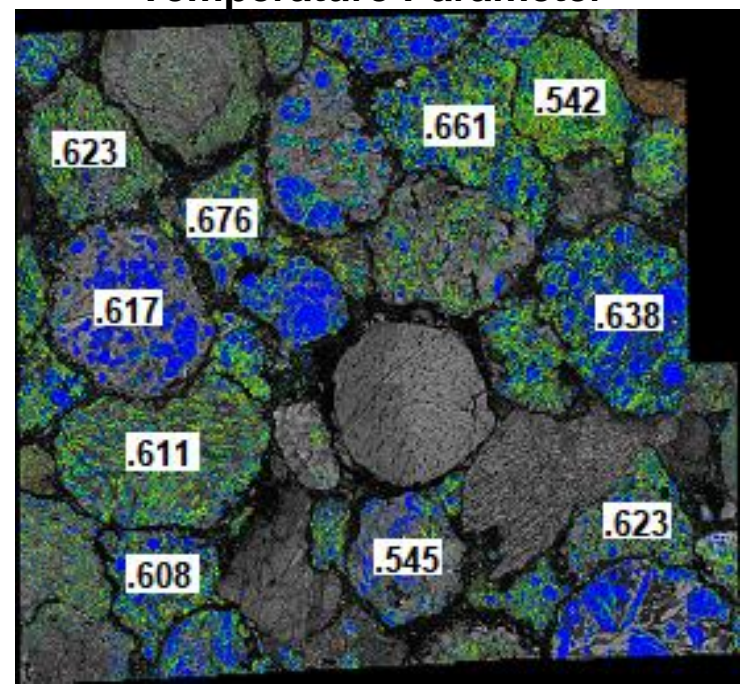

Mean GOS

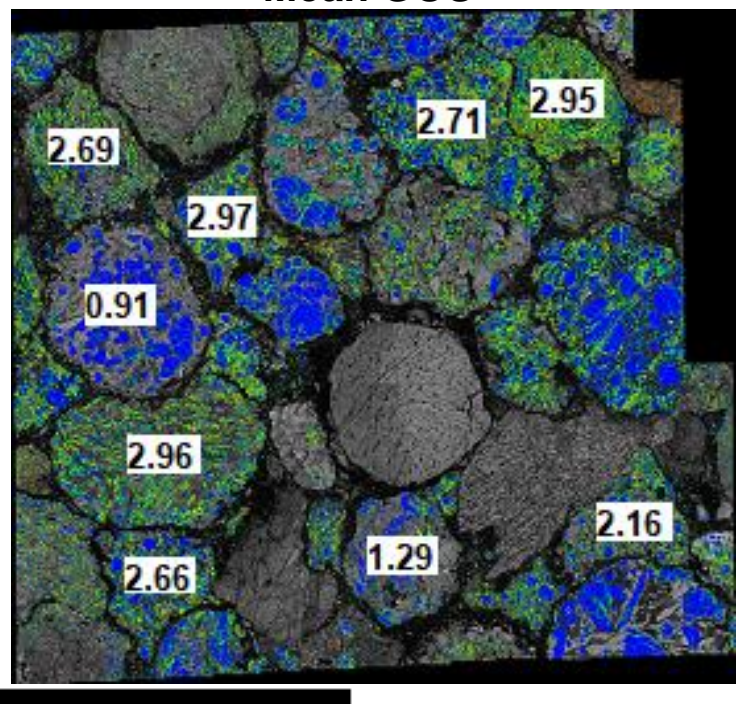

Figure 49. Chondrule Maps of NWA 5421 Map 1222. Upper left map shows chondrule number labels. Upper right map shows $f_{\langle 010\rangle}+f_{\langle 001}>$ values of the labeled chondrules. Lower left map shows shape parameters of the labeled chondrules. Lower right map shows mean GOS values $\left(^{\circ}\right)$ of the labeled chondrules. Background map is the olivine local misorientation map of map 1222. Scale applies to all maps. 
Chondrule ID

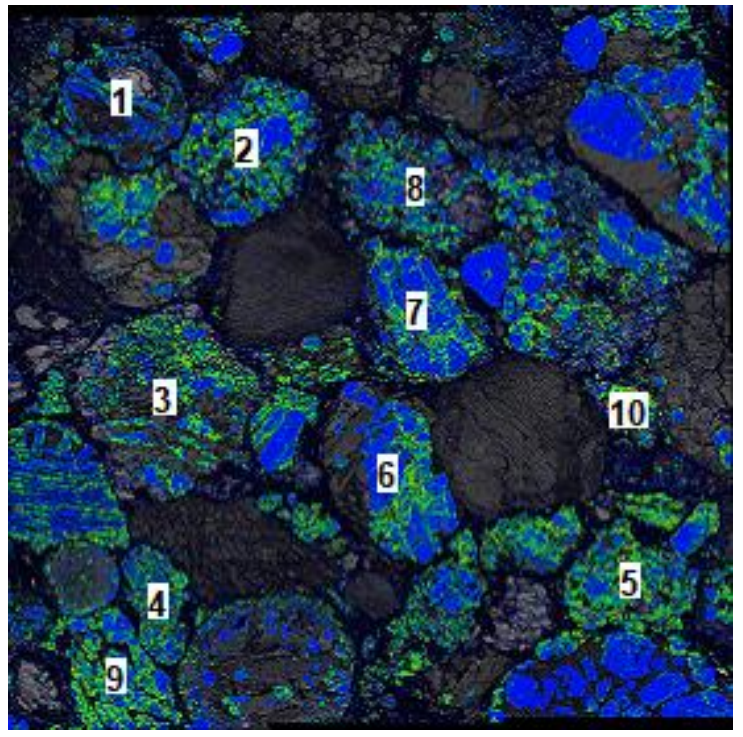

Shape Parameter

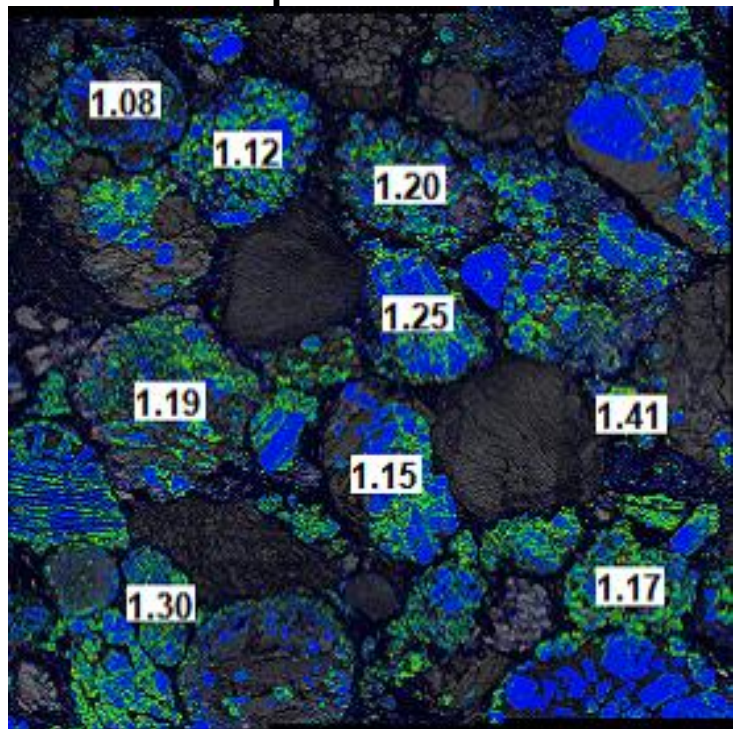

Temperature Parameter

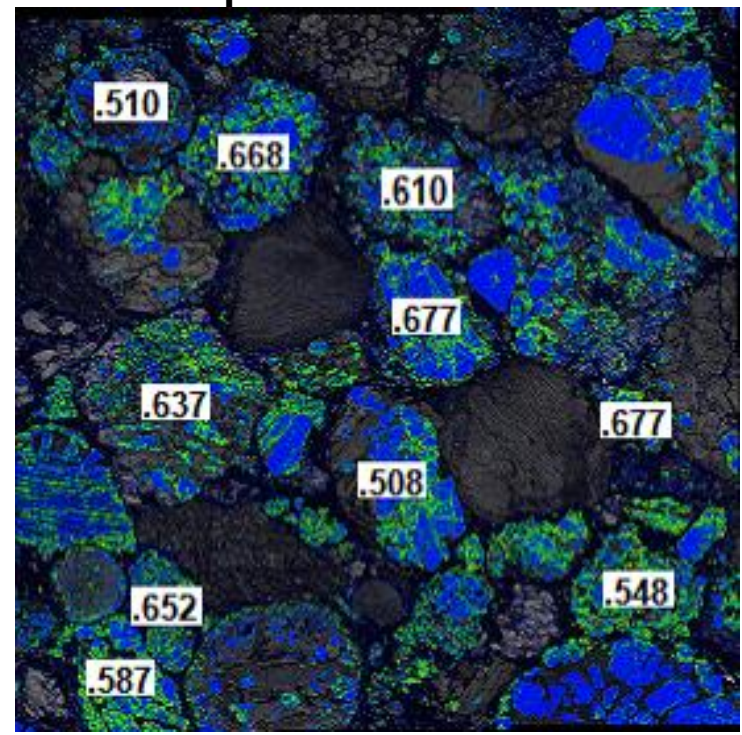

Mean GOS

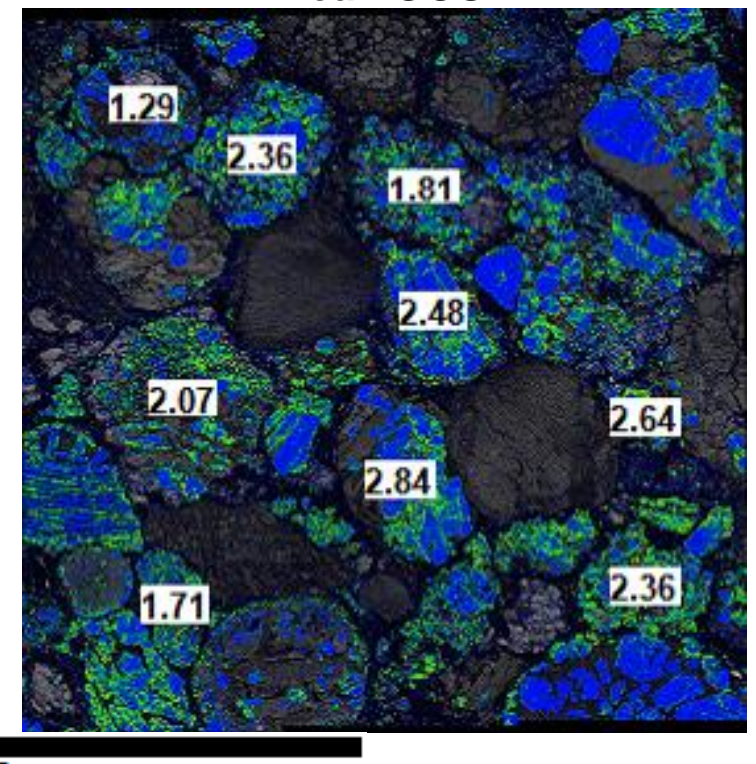

$3250 \mu \mathrm{m}$

Figure 50. Chondrule Maps of NWA 5781 Map 0923. Upper left map shows chondrule number labels. Upper right map shows $f_{\langle 010\rangle}+f_{\langle 001}>$ values of the labeled chondrules. Lower left map shows shape parameters of the labeled chondrules. Lower right map shows mean GOS values $\left(^{\circ}\right)$ of the labeled chondrules. Background map is the olivine local misorientation map of map 0923. Scale applies to all maps. 


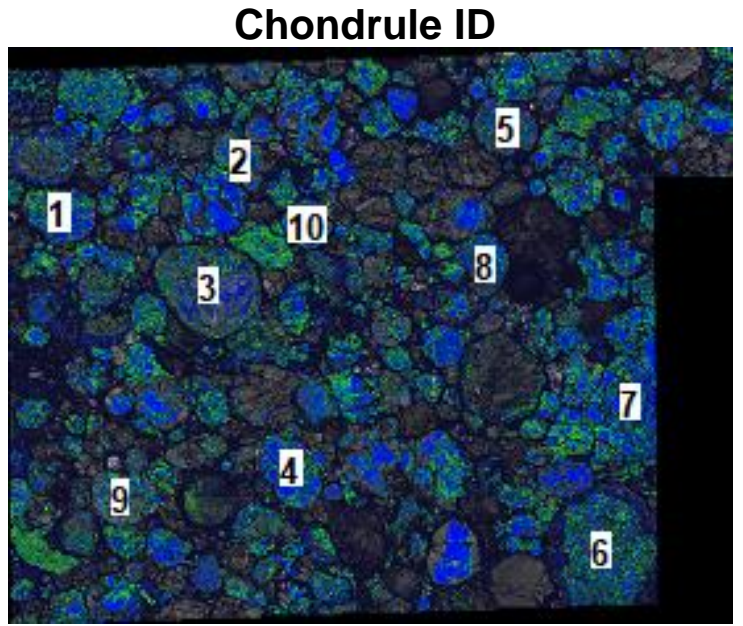

Shape Parameter

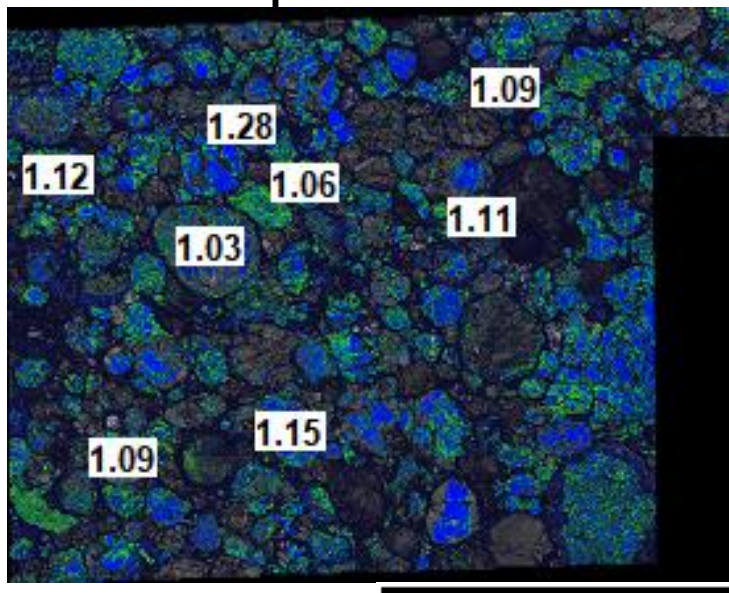

Temperature Parameter

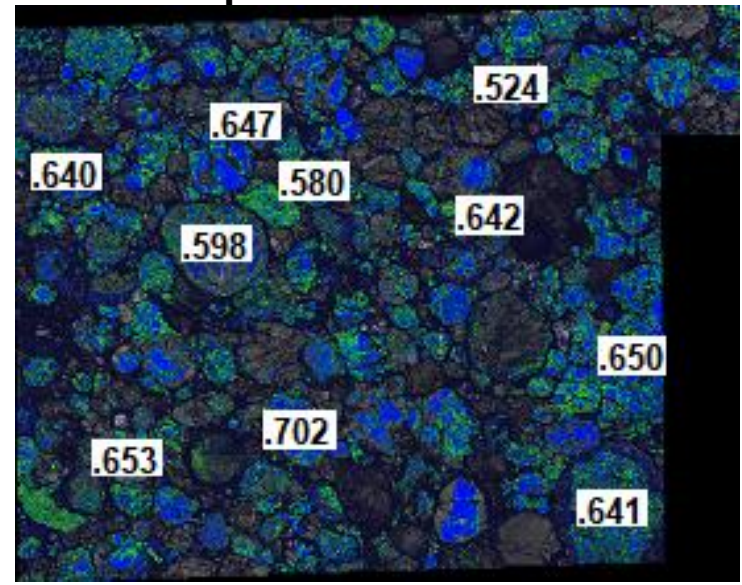

Mean GOS

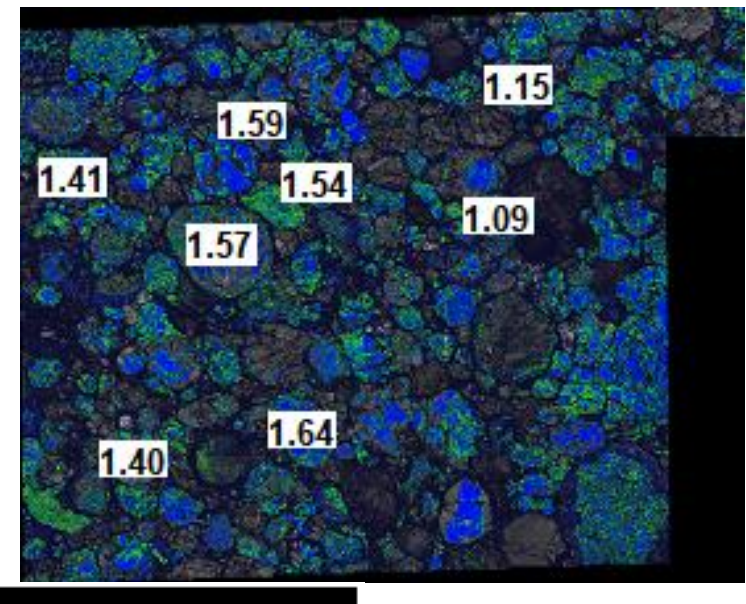

$11000 \mu \mathrm{m}$

Figure 51. Chondrule Maps of NWA 5781 Map 1005. Upper left map shows chondrule number labels. Upper right map shows $\mathbf{f}\langle 010\rangle+\mathbf{f}\langle 001\rangle$ values of the labeled chondrules. Lower left map shows shape parameters of the labeled chondrules. Lower right map shows mean GOS values $\left(^{\circ}\right)$ of the labeled chondrules. Background map is the olivine local misorientation map of map 1005. Scale applies to all maps. 


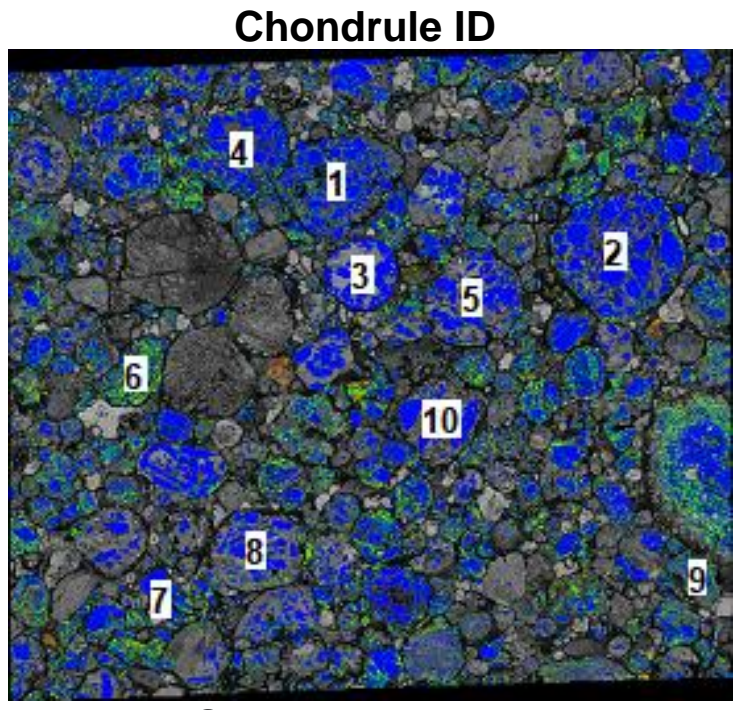

Shape Parameter

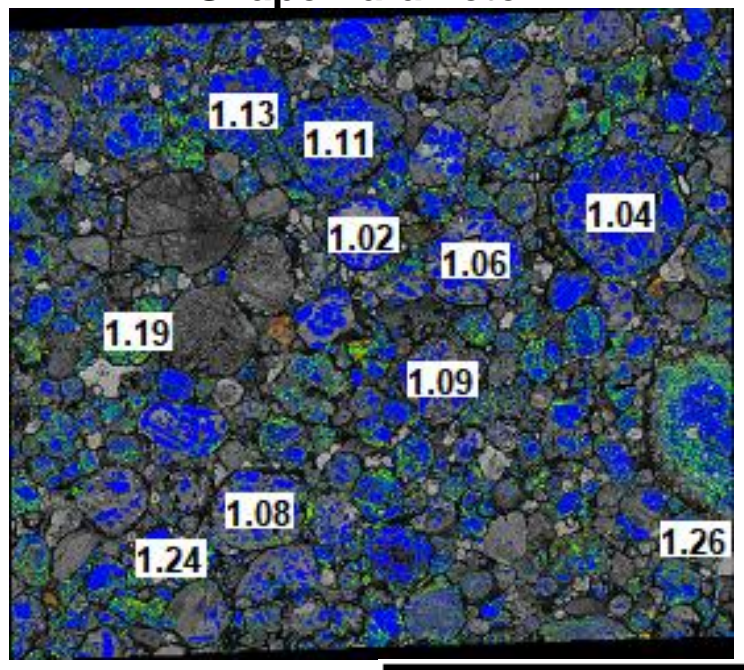

\section{$8500 \mu \mathrm{m}$}

Temperature Parameter

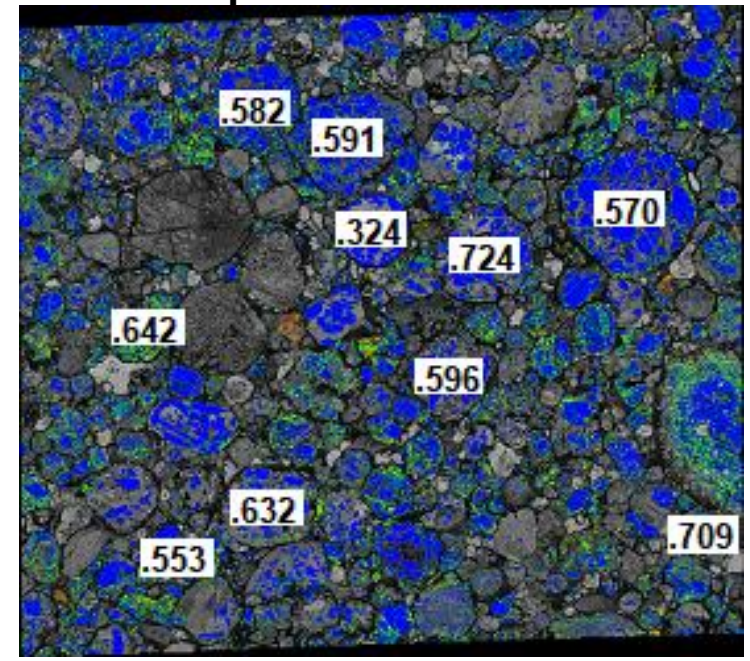

Mean GOS

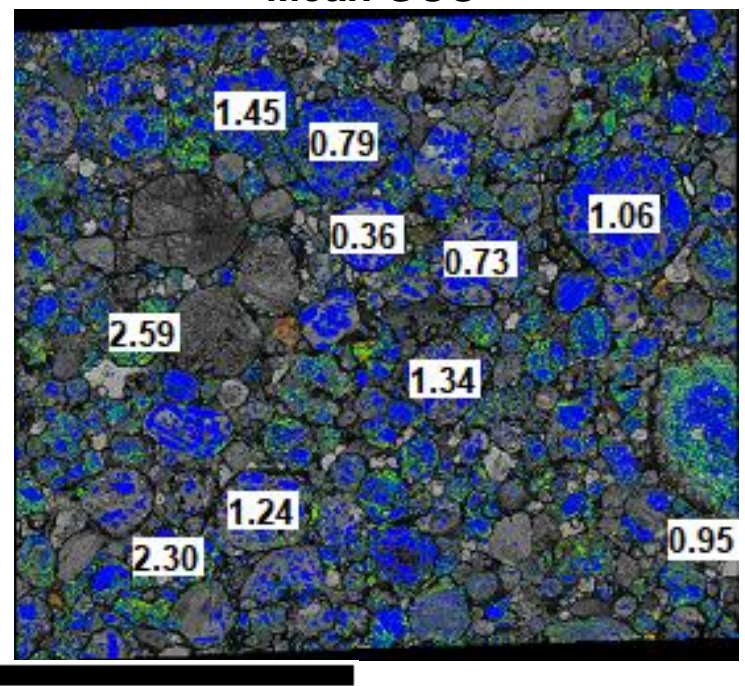

0.95

Figure 52. Chondrule Maps of Tieschitz Map 0208. Upper left map shows chondrule number labels. Upper right map shows $f<010\rangle+\mathbf{f}<001>$ values of the labeled chondrules. Lower left map shows shape parameters of the labeled chondrules. Lower right map shows mean GOS values $\left(^{\circ}\right)$ of the labeled chondrules. Background map is the olivine local misorientation map of map 0208. Scale applies to all maps. 


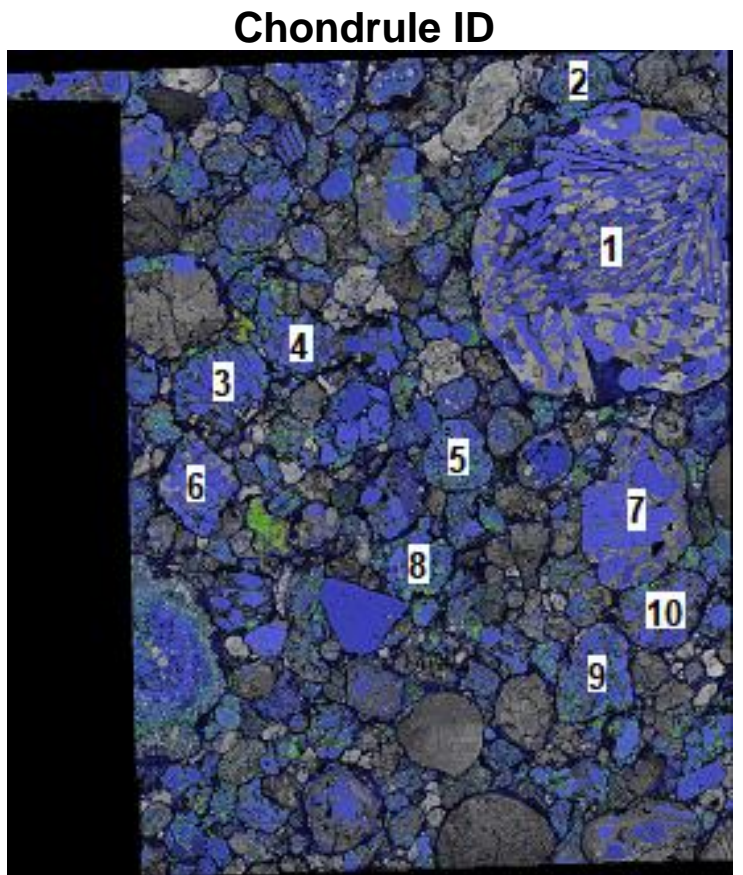

Shape Parameter

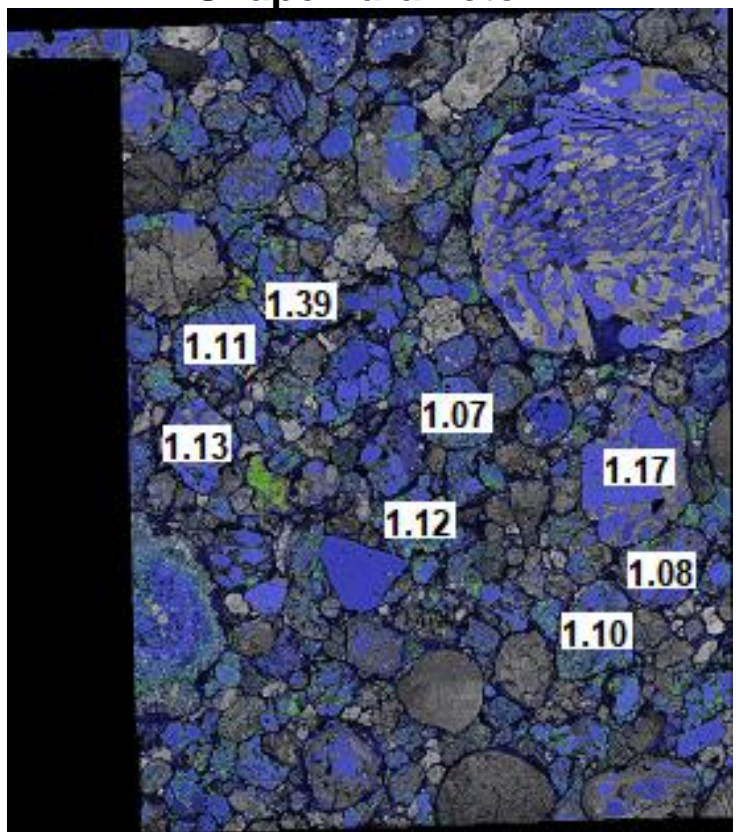

Temperature Parameter

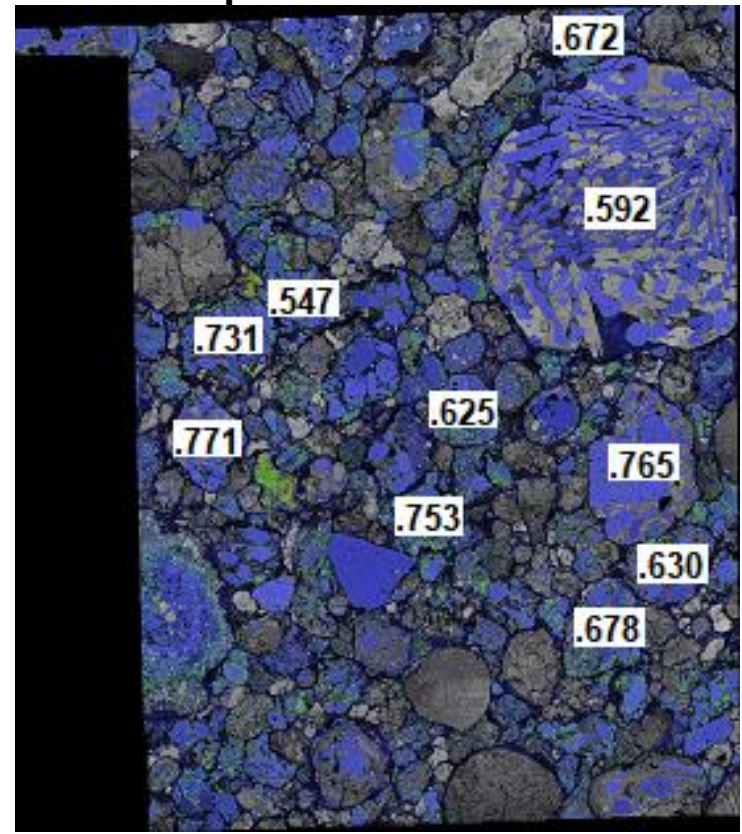

Mean GOS

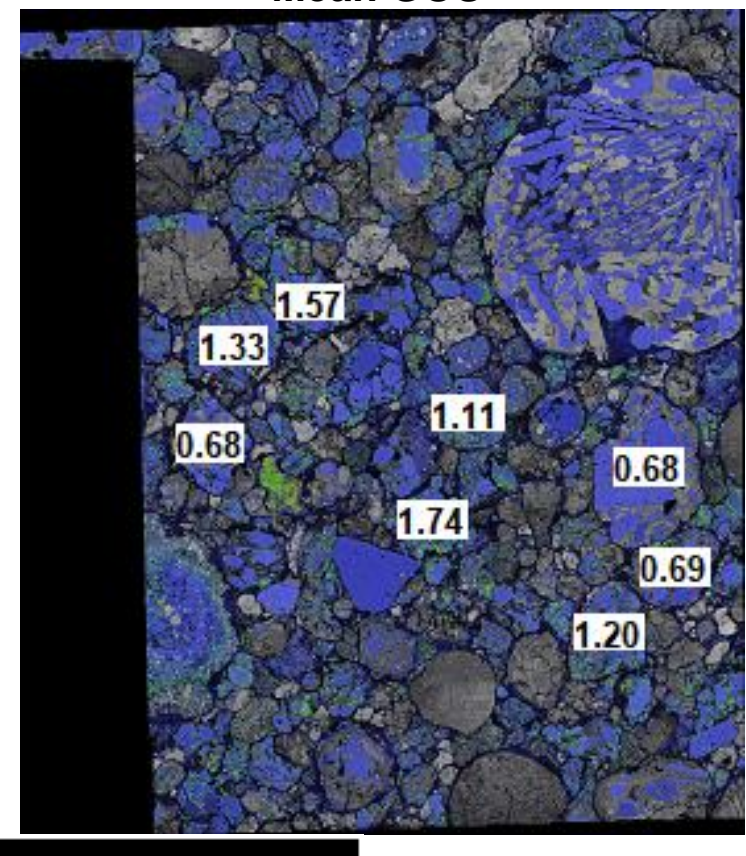

$6750 \mu \mathrm{m}$

Figure 53. Chondrule Maps of Tieschitz Map 0215. Upper left map shows chondrule number labels. Upper right map shows $f_{\langle 010\rangle}+\mathbf{f}<001>$ values of the labeled chondrules. Lower left map shows shape parameters of the labeled chondrules. Lower right map shows mean GOS values $\left(^{\circ}\right)$ of the labeled chondrules. Background map is the olivine local misorientation map of map 0215. Scale applies to all maps. 


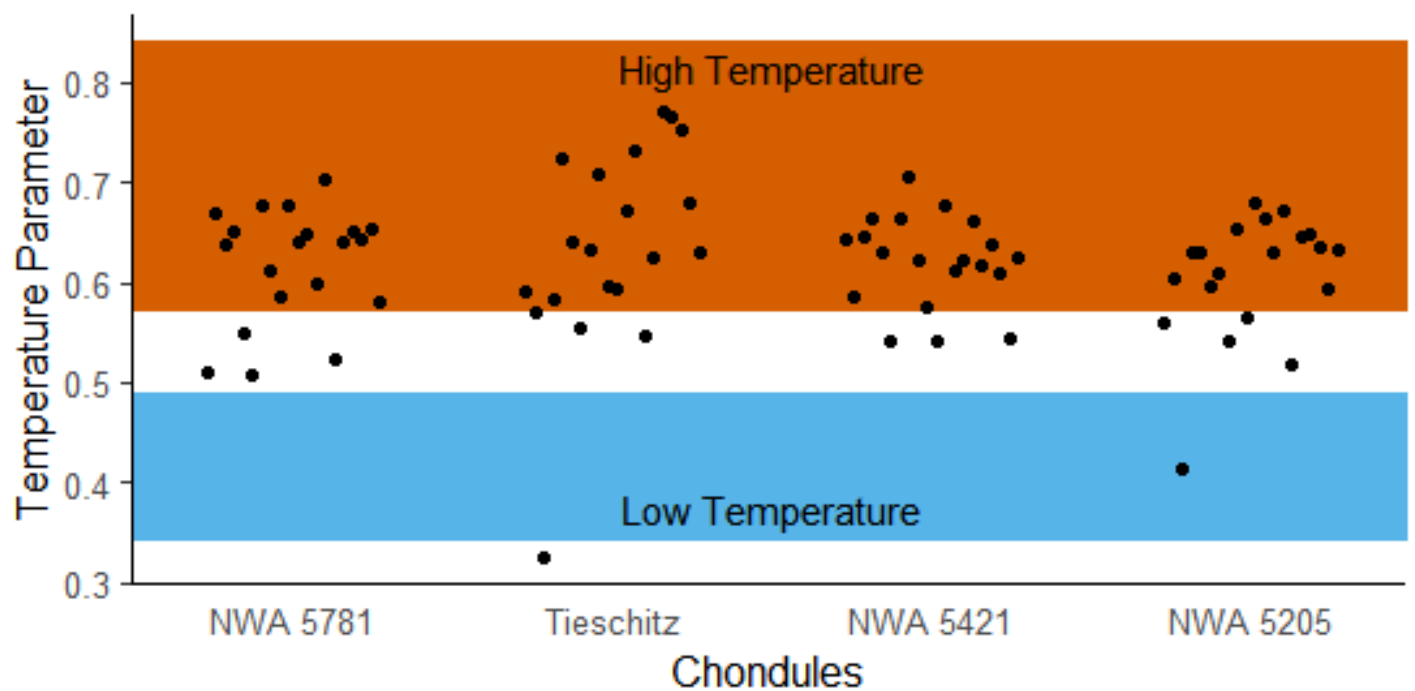

Figure 54. Graph of Chondrule Temperature Parameters. Points are chondrules, grouped by sample. Lower colored region represents the range of observed temperature parameters in cold shocked chondrites in Ruzicka and Hugo (2018). Upper colored region represents the range of observed temperature parameters in hot shocked chondrites in Ruzicka and Hugo (2018). 


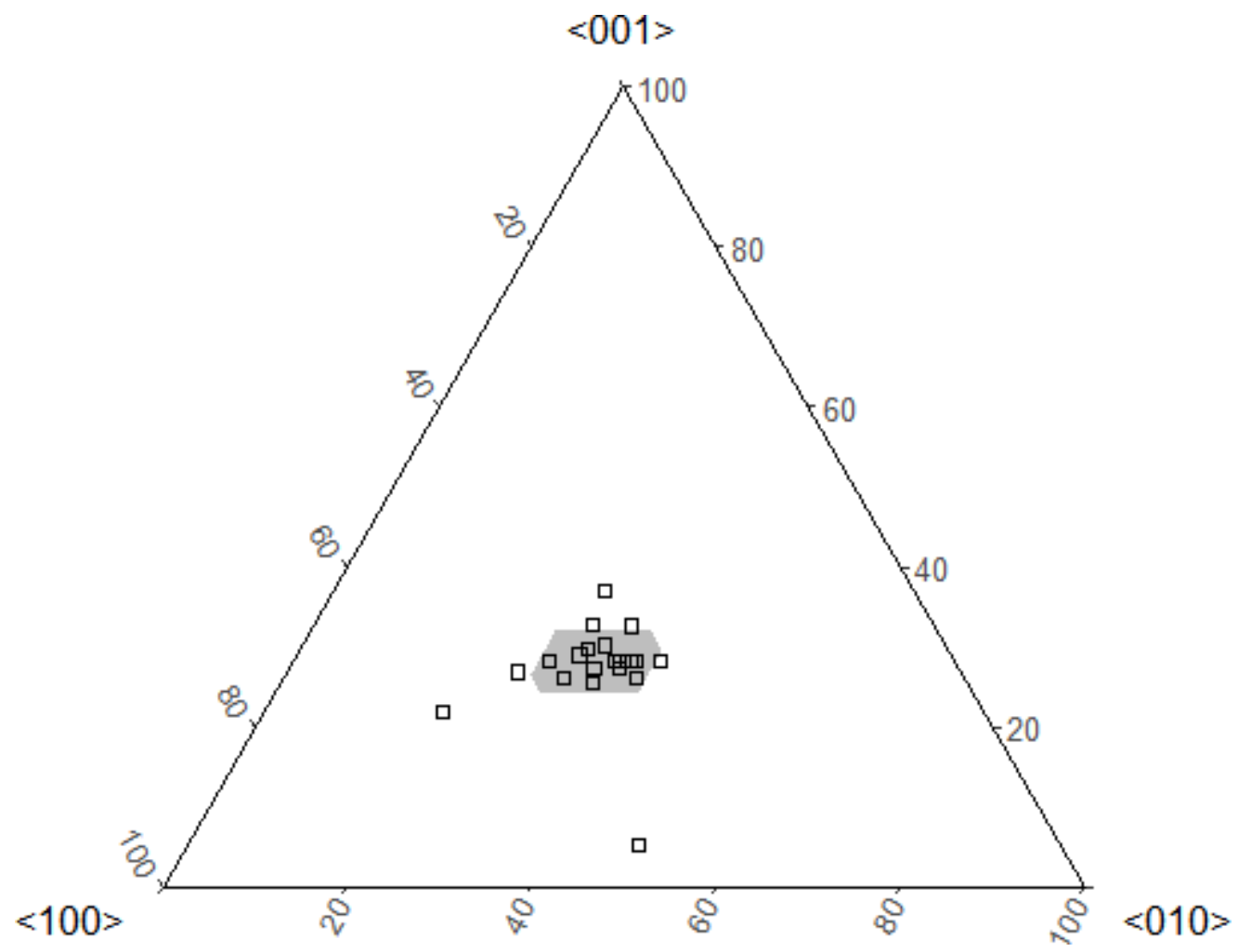

Figure 55. Ternary Displaying Chondrule CRA Data of NWA 5205. Points are chondrules. Gray shaded area represents regional variation determined from regional subsets. 


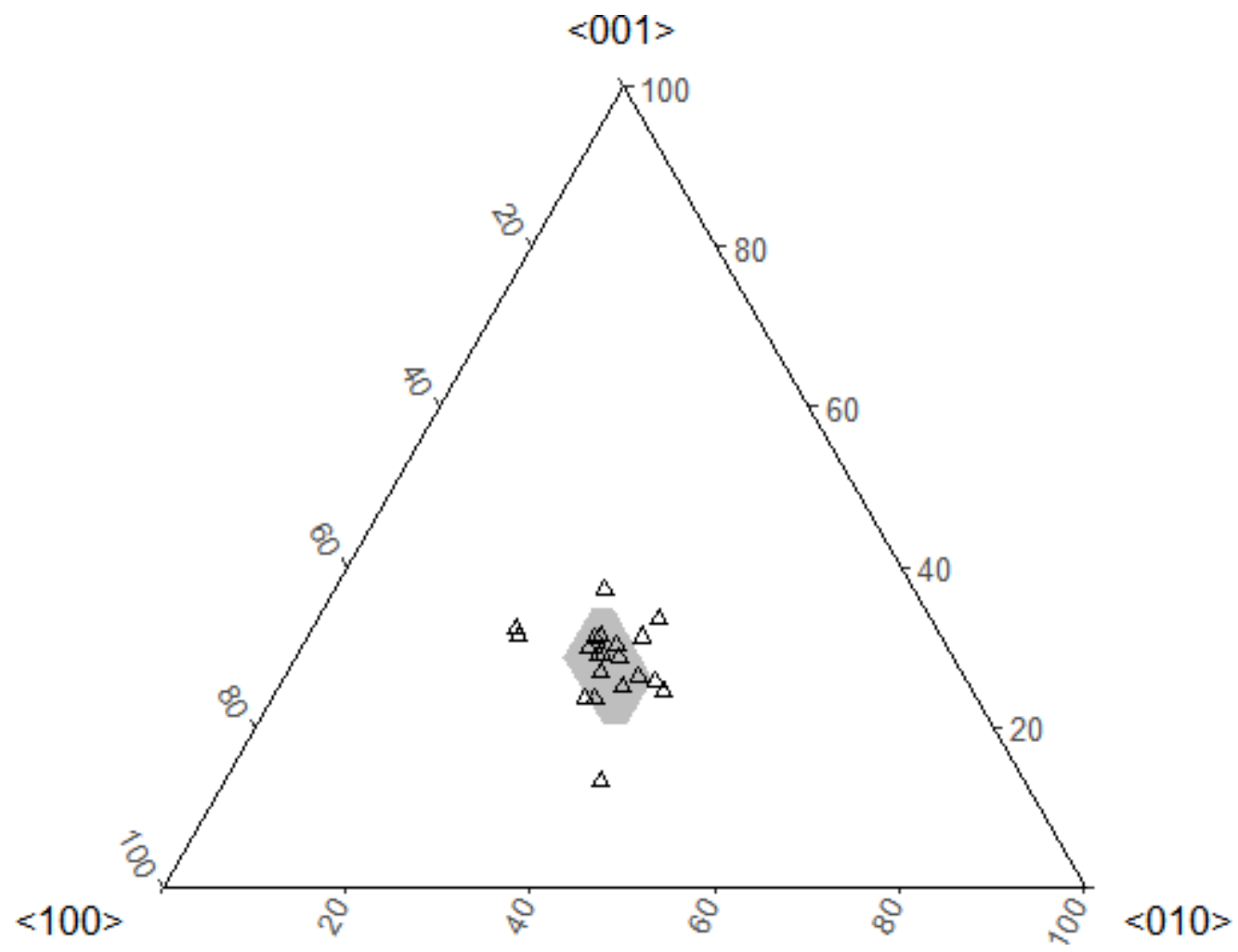

Figure 56. Ternary Displaying Chondrule CRA Data of NWA 5421. Points are chondrules. Gray shaded area represents regional variation determined from regional subsets. 


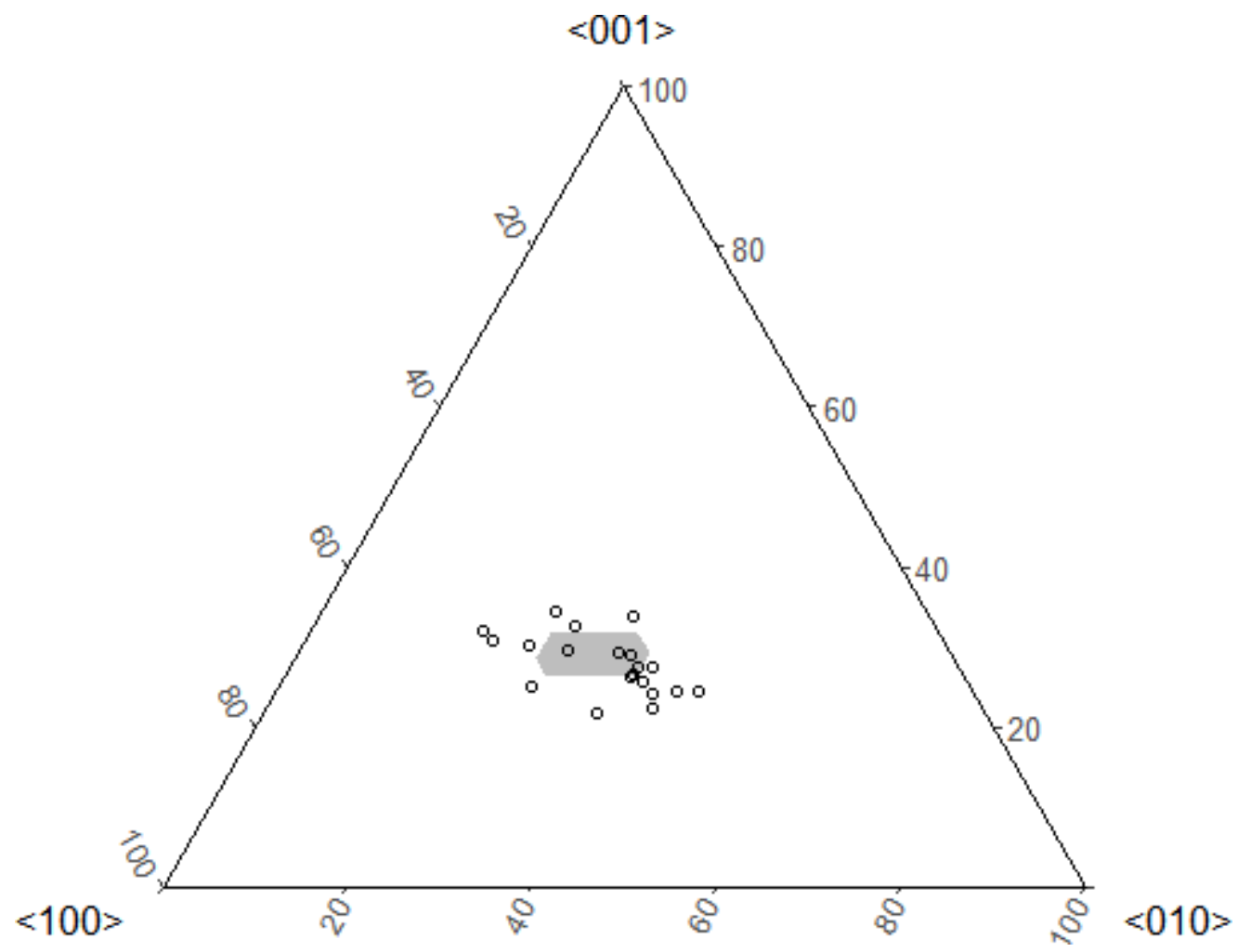

Figure 57. Ternary Displaying Chondrule CRA Data of NWA 5781. Points are chondrules. Gray shaded area represents regional variation determined from regional subsets. 


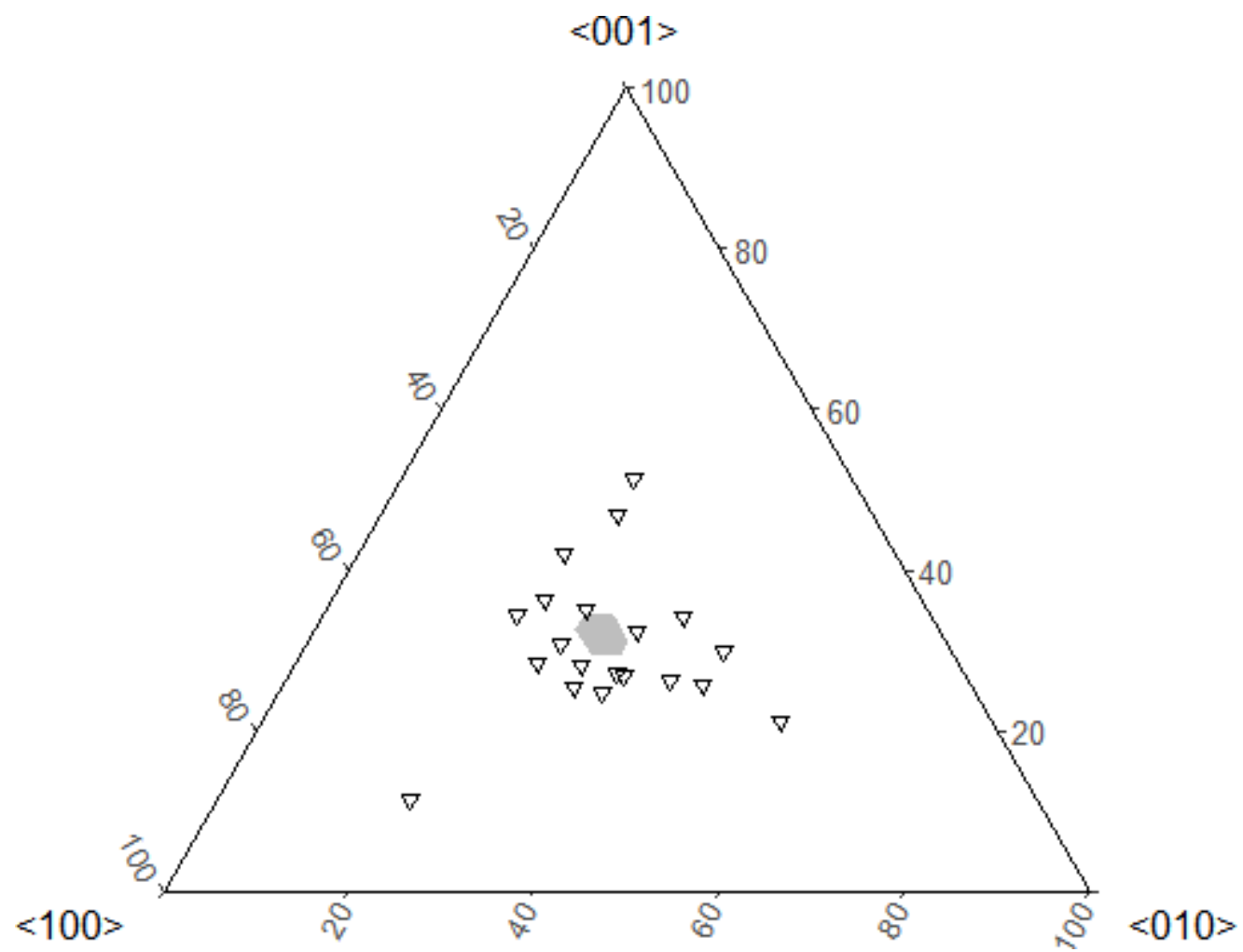

Figure 58. Ternary Displaying Chondrule CRA Data of Tieschitz. Points are chondrules. Gray shaded area represents regional variation determined from regional subsets. Uniquely, regional variation in this sample is determined from two large area maps. Other samples had regional variation determined from a large area map and a small area map. 
NWA 5205

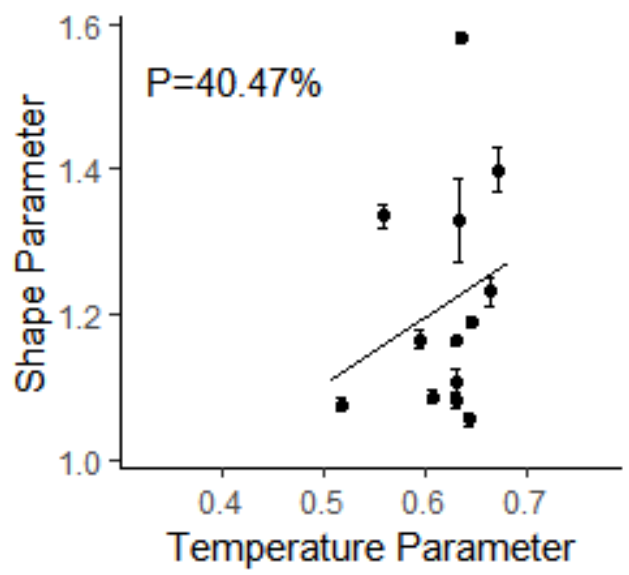

NWA 5781

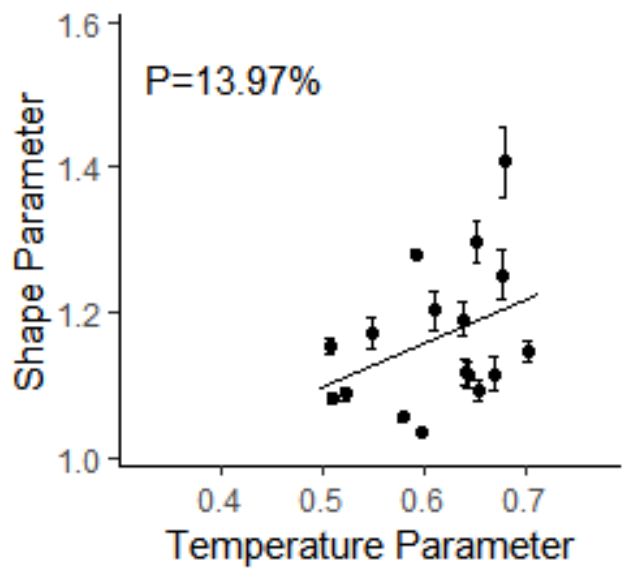

NWA 5421

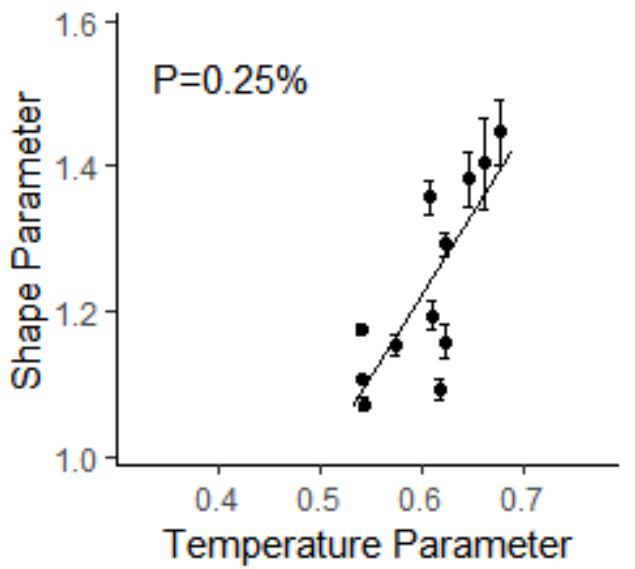

Tieschitz

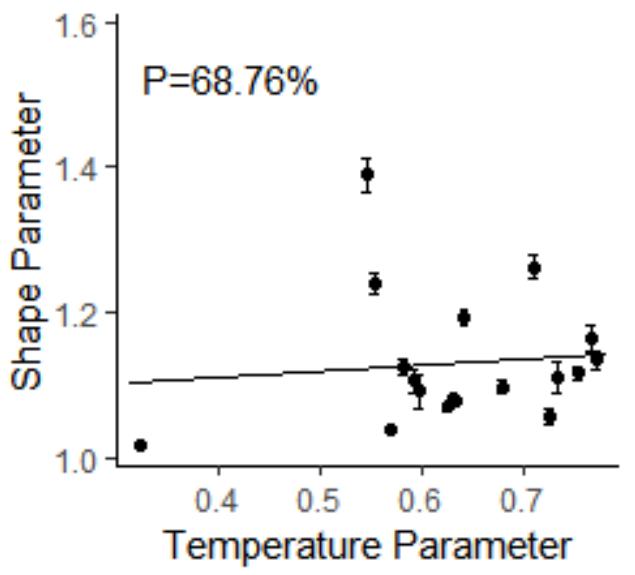

Figure 59. Graphs Comparing Chondrule Temperature and Shape Parameters. "P" is the probability of no significant correlation between the two parameters in the sample, as determined by ANOVA. Points are individual chondrules. Shape parameter error bars are $95 \%$ standard error ranges calculated from five measurements for each chondrule. 

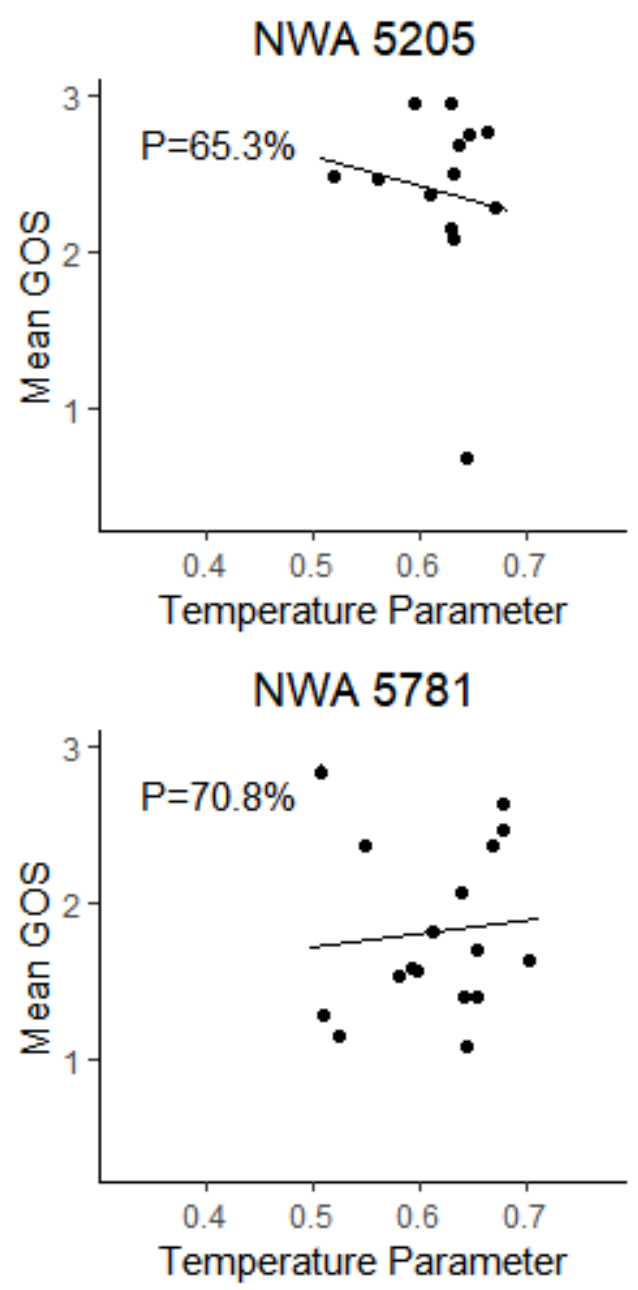
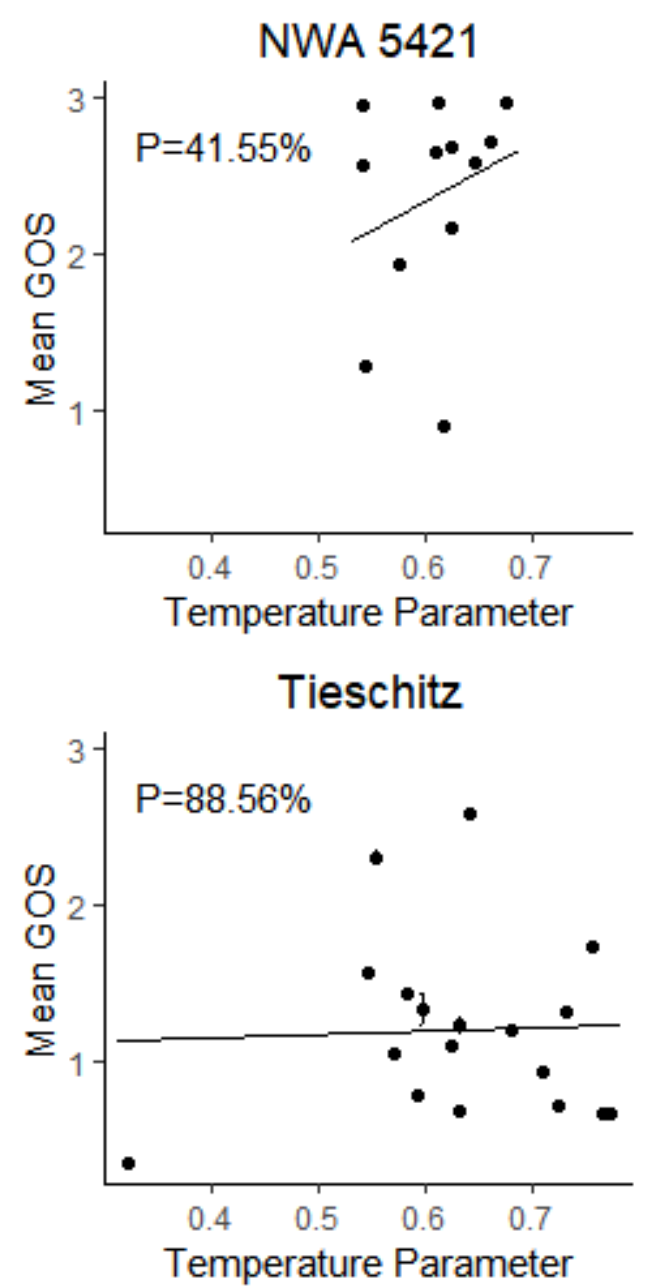

Figure 60. Graphs Comparing Chondrule Temperature Parameters and Mean GOS. "P" is the probability of no significant correlation between the two parameters in the sample, as determined by ANOVA. Points are individual chondrules. 
NWA 5205

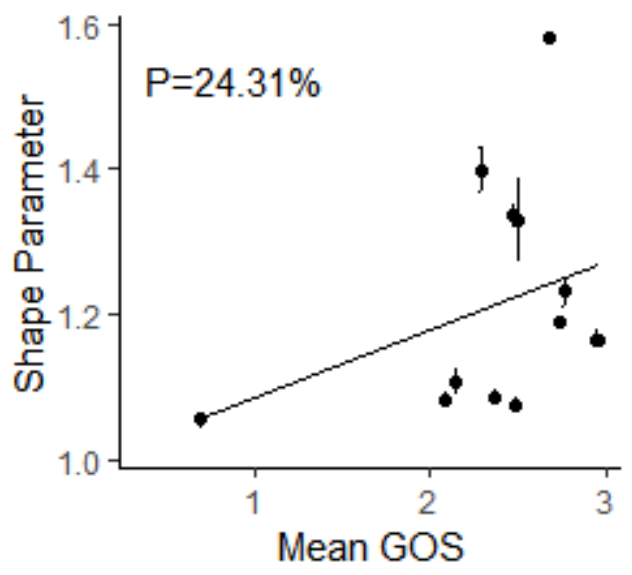

NWA 5781

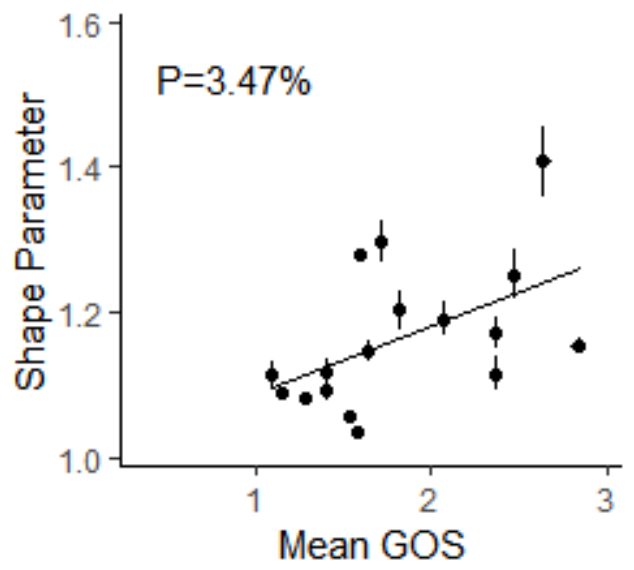

NWA 5421

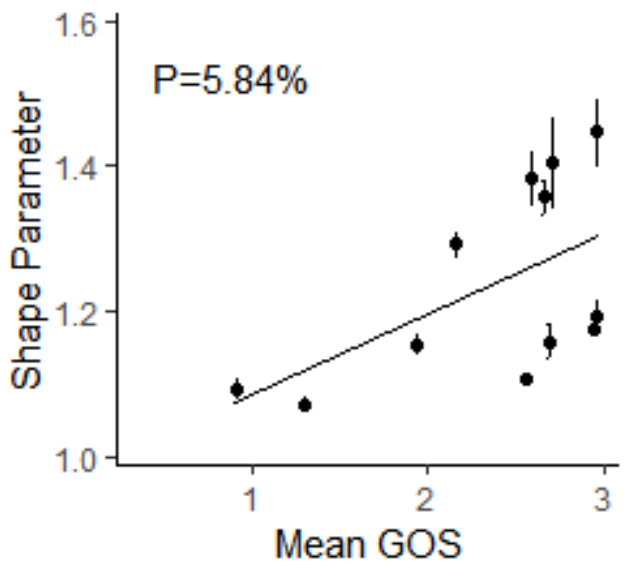

Tieschitz

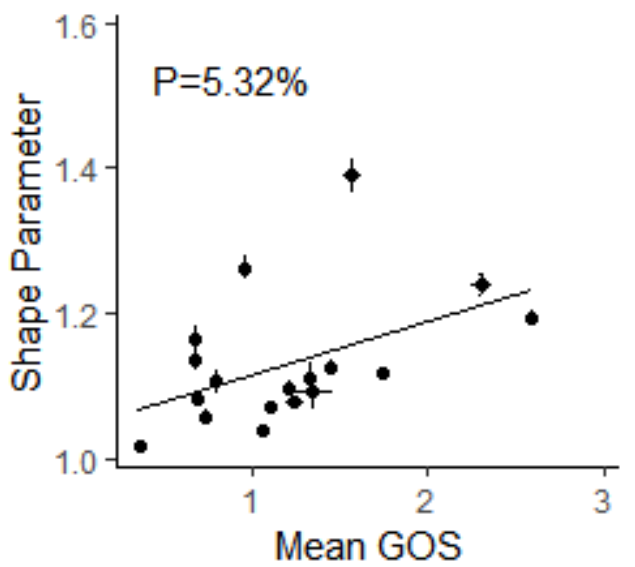

Figure 61. Graphs Comparing Chondrule Mean GOS and Shape Parameters. "P" is the probability of no significant correlation between the two parameters in the sample, as determined by ANOVA. Points are individual chondrules. Shape parameter error bars are $\mathbf{9 5 \%}$ standard error ranges calculated from five measurements for each chondrule. 


\section{Discussion}

\subsection{Anomalous Tieschitz}

A particular item of note is the anomalously high $\mathrm{GOS}_{\text {Mean }} / \mathrm{GOS}_{\text {Median }}$ observed for Tieschitz. All other studied samples show similar data in terms of their GOS Mean $_{\text {GOS }}$ Median values consistent with the low degree of annealing expected for type 3 chondrites. Tieschitz is a significant exception to an otherwise consistent trend that requires further explanation. Tieschitz, as a type 3.6 ordinary chondrite, is unlikely to have significant annealing, especially when NWA 5421 is of a higher metamorphic subtype (3.7) and shows no similar evidence of dislocation repair. If Tieschitz had undergone annealing from metamorphic heating, then NWA 5421 should have as well. As it is not sensible that Tieschitz should be thermally annealed when NWA 5421 is not, there must be another explanation. Recognizing that the apparent annealing parameter is a measure of skewness within a population of GOS measurements, it must be determined what could be responsible for the observed skew if not metamorphic annealing. Observing Figures 5558, it is apparent that the modal activation of slip systems in Tieschitz is more variable than in the other samples. Taking this into account, I could interpret that the observed annealing parameter in Tieschitz is the result of the especially high heterogeneity of the chondrules

of that meteorite as compared to the other samples, and not a result of annealing. Such an interpretation is not unique in the literature, as it was determined by Ruzicka and Hugo (2018) that a high annealing parameter was found in an unannealed breccia (MIL 99301) was created by the heterogeneity between a clast in that meteorite and its surrounding host rock. However, the variances of both weighted stages and mean GOS in Tieschitz are 
unexceptional for the latter and lower than the other samples for the former, which does not support the notion of heterogeneity. Despite this, it may be the case that Tieschitz is a variably shocked and deformed genomict breccia, if the variability in observed shock stages for the rock (S1-3) is considered (Scott et al., 1992; Rubin et al., 1999).

\subsection{Relations between Parameters of Deformation and Temperature}

From the statistical analysis of the chondrules, it is found that there is no significant correlation between the inferred temperatures of the chondrules and any measure of deformation or chondrule shape. It was expected that measures of temperature and deformation would be correlated, as higher temperatures promote plastic deformation. For the unintuitive insignificance of deformational temperature upon deformation, it may be the case that other factors play a more important role in how deformed the chondrules and their olivine grains are. Chondrules and their olivine grains vary in their content of $\mathrm{Mg}$ and other elements, which can affect the liquidus temperatures of these objects. With different liquidus temperatures, chondrules and olivine grains of differing compositions will have different homologous temperatures, and therefore, when at the same temperature may have varying susceptibilities to deformation. Further research comparing olivine and chondrule compositions to their temperature parameters and deformation metrics may be necessary to determine if this is the case. Regardless, the data indicate that deformation is not related to temperature.

As for the deformation metrics, the data suggests there may be a relationship between them. This is indicated by the low ANOVA p-values associated with mean GOS and the shape parameter in three of four samples, although the data does not strictly meet 
the $1.4 \%$ p-value criteria outlined in the methods. This implies that intracrystalline deformation may be linked to chondrule deformation - that when a chondrule is deformed during accretion, at least some of that deformation is accommodated by plastic deformation of its mineral grains. This suggests that EBSD measurable parameters such as GOS can reflect whole chondrule deformation and are related to accretional deformation in the analyzed chondrules. Further, while the measured deformation is unrelated to chondrule deformational temperature, potential chondrule deformation is possibly linked to the plastic deformation within chondrule olivine grains, indicating that temperatures inferred from intracrystalline deformation reflect the codeformational temperature of the chondrule. Grain properties are related to chondrule properties, so grain data could imply the state of chondrules as a whole. Further, this also suggests that the shapes of chondrules in cluster chondrites are at least partly due to deformation, supporting the interpretations of Metzler (2012).

\subsection{Codeformational Heat Source}

Given that a-slip is observed in the samples, it is apparent that many olivine grains in these rocks were at an elevated temperature, above roughly $850^{\circ} \mathrm{C}$ based on the activation temperature of a-slip, during their deformation. The dislocation data indicates that deformation is at least partly a result of high temperature plastic deformation at too high a temperature for liquid water. This allows the removal of pressure solution as a possible origin for the close fit textures. A critical question now is what is the source of the heat? Metamorphism is an unlikely cause, as these samples are all type 3 ordinary chondrites; the heat range implied by the observed slip systems requires temperatures 
inferred for higher metamorphic grades than these rocks have. Shock compaction is also implausible as a heat source, for all the samples used for EBSD analysis are S1, and c-slip dislocations are typically observed for shock related deformation in type 3 chondrites. While shock compaction can heat matrix to high temperature, it does not do the same for chondrules (Davison et al., 2016). One may attempt to argue that these samples have been annealed postshock, but this scenario is unlikely due to the low metamorphic grade of the samples. Further, most of these samples do not have the expected GOS signature of postshock annealing. However, there is potential evidence for a minor degree of annealing in the elevated $\mathrm{GOS}_{\text {mean }} / \mathrm{GOS}_{\text {median }}$ metrics of the NWA samples, possibly from residual heat. A third possible source is residual heat leftover from chondrule formation. Excepting other unknown heat sources, this one is left the most plausible because unlike the other two, it cannot be ruled out.

\subsection{Codeformational Temperature}

Interpreting the CRA data as being reflective of accretion temperature, a complex story emerges. There is strong evidence for hot deformation, or at least, deformation above $850^{\circ} \mathrm{C}$, as most CRA poles for the olivines are consistent with this result. However, there is also some evidence for predominant activation of the cold c-slip systems in a few chondrules, indicating that some were cold. With evidence for both hot and cold deformation during accretion, the most straightforward interpretation is that both hot and cold components were accreted during the formation of these meteorites. This is heterogenous accretion: a mixing of hot and cold components accreting together into one rock or onto the same planetesimal. 
It must be noted however, that another plausible explanation for the two c-slip dominant chondrules exists. If they possess a preferred crystal alignment, as the one in NWA 5205 does due to a barred olivine texture, a stress orientation favoring c-slip can result in the observed slip system activation. In light of this possibility caution toward the idea of heterogeneous accretion is warranted, as the $2.5 \%$ of "cold" chondrules it is based upon cannot be said to be cold without plausible skepticism. It is possible that future work, albeit with a larger sample population of chondrules, may be able to resolve this issue. Regardless, it is clear that the majority of the chondrules in the cluster chondrites accreted hot.

\subsection{Implications for Chondrule Formation Models}

This establishes possible new constraints for chondrule formation models to meet. The first of these requires that accretion of chondrules into a clump or onto a planetesimal be possible soon after the chondrules have been heated, which is supported by the strong evidence for hot accretion observed in this study. The second of these is heterogenous mixing; that a majority of hot chondrules must be able to mix with a small fraction of cold chondrules and then accrete together. This second constraint is less well founded than rapid accretion, as the evidence for cold-accreted chondrules in cluster chondrites is significantly weaker than that for the hot-accreted chondrules. This is because only 2 of 80 of the observed chondrules were deformed while cold, and furthermore the interpreted cold deformation of them can be reasonably doubted.

The constraint of rapid accretion is the more limiting constraint. Density shockwaves are able to concentrate chondrules and thereby facilitate rapid accretion, 
especially in a model where chondrules are already concentrated in a dense cloud or rubble pile of chondrules, such as that of Ruzicka (2012). Bow shock models are well able to meet the rapid accretion constraint, as an object for the chondrules to accrete on is available in the model. Impact models are also compliant with the new rapid accretion constraint. This is because in these models there are necessarily one or two colliding planetesimals nearby for chondrules to quickly accrete onto. In the lightning models, there is little reason for heating and accretion to be associated on the requisite timescales, creating a difficulty for meeting the rapid accretion constraint. Explaining how heating could be closely associated with accretion is difficult in the current sheet model, as there is no reason in the models for chondrules be become concentrated into a clump and no close proximity to a planetesimal is required by the model. The $\mathrm{X}$-wind is incompatible with the rapid accretion constraint, as there is no reason for accretion to follow shortly after heating. In the gamma ray burst model there is no reason for accretion or clumping to closely follow heating.

The heterogenous mixing constraint is less restricting than the rapid accretion constraint. Density shockwave models have difficulty meeting the constraint. These shockwaves heat vast regions of space, so it is difficult to explain the inferred mixing of hot and cold chondrules. Bow shocks process a smaller region of space, and so conceivably allow for freshly heated chondrules to mix with colder chondrules in orbit of or in the wake of the protoplanet or planetesimal. However, bow shock models are better able to meet the mixing constraint if the shockwave generating object is a protoplanet instead of a planetesimal, as the bow shocks of the latter are so small that chondrules will not be entrained into the gas flow, and thereby collide with the object. In the impact models, it is 
plausible that different debris clouds may cool at different rates and at variable rates throughout themselves, allowing for the mixing of cool and hot chondrules. Within the lightning models, the close proximity of hot and cold chondrules is easy to justify, given the small volumes of space that are heated. In the current sheet model, it is easy to explain the mixing of hot and cold chondrules with the thin volumes of the heated regions. The Xwind model's extensive region of heating and the trajectory of chondrules out of the protoplanetary disk are incompatible with the mixing of hot and cold components. The gamma ray burst model heats the whole of the solar nebula, but as it is conceivable that solids could be shielded by planetesimals or other large objects, a mixing of hot and cold chondrules after heating is not implausible. However, such cold chondrules would have be created by prior heating events, which is difficult to justify with the model, and this is ignoring the issues of how hot and cold chondrules would be mixed quickly and efficiently.

In summary, only protoplanetary shockwave models and impact models are compatible with both of the new constraints. In the case that the constraint of heterogenous mixing can be dismissed, density shockwaves can be viable. Other models cannot be made compatible with rapid accretion, and so are not viable, regardless of whether heterogenous mixing is a valid constraint or not. 
Table 12. Summary of Implications on Formation Models for New Constraints. " $X$ " indicates the model is compatible with the constraint with no or little modification. Incompatibility is indicated by leaving the space blank.

Model

Density Shockwaves

Protoplanetary Bow Shockwaves

Large Impacts

Lightning

Current Sheets

X-Wind

Gamma Ray Burst
Rapid Accretion Heterogenous Mixing $\mathrm{X}$

$\mathrm{X} \quad \mathrm{X}$

$\mathrm{X} \quad \mathrm{X}$

X

X 


\section{Conclusions}

In an analysis of four unshocked type 3 ordinary chondrites with cluster chondrite lithologies, evidence for temperature at accretion was found from the temperaturedependent activation of olivine dislocation slip systems that accommodate the deformation in these rocks. Strong evidence for primarily hot accretion of chondrules is observed from inferred slip system activation in their olivine grains. While primarily hot, a mixture of temperature signals is found, indicating a possibly heterogenous mixture of cold and hot chondrules accreting together to form the studied cluster chondrites. However, $97.5 \%$ of analyzed chondrules were hot during accretion, and the small number of cold-accreted chondrules can be reasonably questioned. Temperature of the chondrules is not well correlated with their degree of deformation, but their intracrystalline and whole-chondrule deformation are possibly linked. The most plausible source of heating for the accreted hot chondrules is the unknown chondrule formation mechanism, as these objects could not have been heated by thermal metamorphism or shock metamorphism in these unshocked and unequilibrated chondrites. This establishes two new possible constraints for chondrule formation mechanisms: 1) they must allow the mixing of hot and cold chondrules and 2) they must allow for chondrule accretion to be spatially and temporally proximal to chondrule formation. The second of these is founded on the weak evidence for coldaccreted chondrules, but the former is well supported by the data for hot chondrule deformation. Bow shock and impact models for chondrule formation can most plausibly meet these new constraints, whereas density shockwaves can possibly meet the rapid accretion constraint. Other models require modification to meet the constraints, 
particularly the rapid accretion constraint, and potentially must be rejected if they cannot be so accommodated. 


\section{References}

Alexander, C.M.O., Barber, D.J., and Hutchison, R., 1989, The microstructure of Semarkona and Bishunpur: Geochimica et Cosmochimica Acta, v. 53, p. 30453057, doi: 10.1016/0016-7037(89)90180-4.

Alexander, C.M.O., and Ebel, D.S., 2012, Questions, questions: Can the contradictions between the petrologic, isotopic, thermodynamic, and astrophysical constraints on chondrule formation be resolved? Meteoritics and Planetary Science, v. 47, p. 11571175, doi: 10.1111/j.1945-5100.2011.01308.x.

Alexander, C.M.O., Hutchison, R., and Barber, D.J., 1989, Origin of chondrule rims and interchondrule matrices in unequilibrated ordinary chondrites: Earth and Planetary Science Letters, v. 95, p. 187-207, doi: 10.1016/0012-821X(89)90096-4.

Alexander, C.M.O., and Wang, J., 2001, Iron isotopes in chondrules: Implications for the role of evaporation during chondrule formation: Meteoritics and Planetary Science, v. 36, p. 419-428, doi: 10.1111/j.1945-5100.2001.tb01883.x.

Amelin, Y., and Krot, A., 2007, Pb isotopic age of the Allende chondrules: Meteoritics and Planetary Science, v. 42, p. 1321-1335, doi: 10.1111/j.19455100.2007.tb00577.x.

Amelin, Y., Krot, A.N., Hutcheon, I.D., and Ulyanov, A.A., 2002, Lead isotopic ages of chondrules and calcium-aluminum - rich inclusions: Science, v. 297, p. 1678-1684, doi: $10.1126 /$ science. 1073950 .

Arakawa, S., and Nakamoto, T., 2016, Compound chondrule formation via collision of supercooled droplets: Icarus, v. 276, p. 102-106, doi: 10.1016/j.icarus.2016.04.041.

Ashworth, J.R., Barber, D.J., Brown, G.M., and Smith, J. V., 1977, Electron microscopy of some stony meteorites [and discussion]: Philosophical Transactions of the Royal Society A: Mathematical, Physical and Engineering Sciences, v. 286, p. 493-506, doi: 10.1098/rsta.1977.0128.

Ashworth, J.R., and Mallinson, L.G., 1985, Transmission electron microscopy of L-group chondrites, 2. Experimentally annealed Kyushu: Earth and Planetary Science Letters, v. 73, p. 33-40, doi: 10.1016/0012-821X(85)90032-9.

Asphaug, E., Jutzi, M., and Movshovitz, N., 2011, Chondrule formation during planetesimal accretion: Earth and Planetary Science Letters, v. 308, p. 369-379, doi: 10.1016/j.eps1.2011.06.007.

Baecker, B., Rubin, A.E., and Wasson, J.T., 2017, Secondary melting events in Semarkona chondrules revealed by compositional zoning in low-Ca pyroxene: Geochimica et Cosmochimica Acta, v. 211, p. 256-279, doi: 10.1016/j.gca.2017.05.013.

Baker, J., Bizzarro, M., Wittig, N., Connelly, J., and Haack, H., 2005, Early planetesimal melting from an age of $4.5662 \mathrm{Gyr}$ for differentiated meteorites: Nature, v. 436, p. 1127-1131, doi: 10.1038/nature03882.

Becker, M., Hezel, D.C., Schulz, T., Elfers, B.-M., and Münker, C., 2015, Formation timescales of CV chondrites from component specific Hf-W systematics: Earth and Planetary Science Letters, v. 432, p. 472-482, doi: 10.1016/j.epsl.2015.09.049.

Bizzarro, M., Connelly, J.N., and Krot, A.N., 2017, Chondrules: Ubiquitous chondritic 
solids tracking the evolution of the Solar protoplanetary disk, in Pessah, M. and Gressel, O. eds., Formation, Evolution, and Dynamics of Young Solar Systems, p. 161-195, doi: 10.1007/978-3-319-60609-5.

Bland, P.A., Alard, O., Benedix, G.K., Kearsley, A.T., Menzies, O.N., Watt, L.E., and Rogers, N.W., 2005, Volatile fractionation in the early solar system and chondrule/matrix complementarity: Proceedings of the National Academy of Sciences, v. 102, p. 13755-13760, doi: 10.1073/pnas.0501885102.

Bland, P.A., Collins, G.S., Davison, T.M., Abreu, N.M., Ciesla, F.J., Muxworthy, A.R., and Moore, J., 2014, Pressure-temperature evolution of primordial solar system solids during impact-induced compaction: Nature Communications, v. 5, p. 1-13, doi: $10.1038 /$ ncomms6451.

Boley, A.C., and Durisen, R.H., 2008, Gravitational Instabilities, Chondrule Formation, and the FU Orionis Phenomenon: Astrophysical Journal, v. 685, p. 1193-1209, doi: 10.1086/591013.

Bollard, J., Connelly, J.N., and Bizzarro, M., 2015, Pb-Pb dating of individual chondrules from the $\mathrm{CBa}$ chondrite Gujba: Assessment of the impact plume formation model: Meteoritics and Planetary Science, v. 50, p. 1197-1216, doi: 10.1111/maps.12461.

Bollard, J., Connelly, J.N., Whitehouse, M.J., Pringle, E.A., Bonal, L., Jørgensen, J.K., Nordlund, A., Moynier, F., and Bizzarro, M., 2017, Early formation of planetary building blocks inferred from Pb isotopic ages of chondrules: Science Advances, v. 3, p. 1-9, doi: 10.1126/sciadv.1700407.

Boss, A.P., 1996, A concise guide to chondrule formation models, in Hewins, R.H., Jones, R.H., and Scott, E.R.D. eds., Chondrules and the Protoplanetary Disk, Cambridge, Cambridge University Press, p. 257-263.

Boss, A.P., 2000, Possible rapid gas giant planet formation in the solar nebula and other protoplanetary disks: The Astrophysical Journal, v. 536, p. L101-L104, doi: $10.1086 / 312737$.

Boss, A.P., and Durisen, R.H., 2005a, Chondrule-forming shock fronts in the solar nebula: A possible unified scenario for planet and chondrite formation: The Astrophysical Journal, v. 621, p. L137-L140, doi: 10.1086/429160.

Boss, A.P., and Durisen, R.H., 2005b, Sources of shock waves in the protoplanetary disk, in Chondrites and the Protoplanetary Disk, p. 821-838.

Bouvier, A., Gattacceca, J., Agee, C., Grossman, J., and Metzler, K., 2017, The Meteoritical Bulletin, No. 104: Meteoritics and Planetary Science, v. 1, p. 1-247, doi: 10.1111/maps.12930.

Brearley, A.J., 1990, Carbon-rich aggregates in type 3 ordinary chondrites: Characterization, origins, and thermal history: Geochimica et Cosmochimica Acta, v. 54, p. 831-850, doi: 10.1016/0016-7037(90)90377-W.

Budde, G., Burkhardt, C., Brennecka, G.A., Fischer-Gödde, M., Kruijer, T.S., and Kleine, T., 2016, Molybdenum isotopic evidence for the origin of chondrules and a distinct genetic heritage of carbonaceous and non-carbonaceous meteorites: Earth and Planetary Science Letters, v. 454, p. 293-303, doi: 10.1016/j.epsl.2016.09.020.

Budde, G., Kruijer, T.S., and Kleine, T., 2018, Hf-W chronology of CR chondrites: Implications for the timescales of chondrule formation and the distribution of $26 \mathrm{Al}$ in 
the solar nebula: Geochimica et Cosmochimica Acta, v. 222, p. 284-304, doi: 10.1016/j.gca.2017.10.014.

Cain, P.M., McSween, H.Y.J., and Woodward, N.B., 1986, Structural deformation of the Leoville chondrite: Earth and Planetary Science Letters, v. 77, p. 165-175.

Carter, N.L., 1971, Static deformation of silica and silicates: Journal of Geophysical Research, v. 76, p. 5514-5540, doi: 10.1029/JB076i023p05514.

Carter, N.L., and Ave'Lallemant, H.G., 1970, High temperature flow of dunite and peridotite: Geological Society of America Bulletin, v. 81, p. 2181-2202.

Carter, N.L., Decarli, P.S., and Raleigh, C.B., 1968, Deformation of olivine in stony meteorites: Journal of Geophysical Research, v. 73, p. 5439-5461, doi: 10.1029/JB073i016p05439.

Charles, C.R.J., Robin, P.Y.F., Davis, D.W., and McCausland, P.J.A., 2018, Shapes of chondrules determined from the petrofabric of the CR2 chondrite NWA 801: Meteoritics and Planetary Science, v. 53, p. 935-951, doi: 10.1111/maps.13038.

Chaumard, N., Humayun, M., Zanda, B., and Hewins, R.H., 2018, Cooling rates of type I chondrules from Renazzo: Implications for chondrule formation: Meteoritics and Planetary Science, v. 53, p. 984-1005, doi: 10.1111/maps.13040.

Ciesla, F.J., 2005, Chondrule-forming processes - An overview, in Chondrites and the Protoplanetary Disk, p. 811-820.

Ciesla, F.J., 2006, Chondrule collisions in shock waves: Meteoritics and Planetary Science, v. 41, p. 1347-1359, doi: 10.1111/j.1945-5100.2006.tb00526.x.

Ciesla, F.J., and Hood, L.L., 2002, The nebular shock wave model for chondrule formation: Shock processing in a particle-gas suspension: Icarus, v. 158, p. 281-293, doi: 10.1006/icar.2002.6895.

Ciesla, F.J., Lauretta, D.S., and Hood, L.L., 2004, The frequency of compound chondrules and implications for chondrule formation: Meteoritics and Planetary Science, v. 39, p. 531-544, doi: 10.1111/j.1945-5100.2004.tb00917.x.

Clarke, R.S.J., 1975, The Meteoritical Bulletin, No. 53: Meteoritics, v. 10, p. 133-158.

Cohen, B.A., and Hewins, R.H., 2004, An experimental study of the formation of metallic iron in chondrules: Geochimica et Cosmochimica Acta, v. 68, p. 16771689, doi: 10.1016/j.gca.2003.10.004.

Cohen, B.A., Hewins, R.H., and Yu, Y., 2000, Evaporation in the young solar nebula as the origin of "just-right" melting of chondrules: Nature, v. 406, p. 600-602, doi: $10.1038 / 35020514$.

Connelly, J.N., Amelin, Y., Krot, A.N., and Bizzarro, M., 2008, Chronology of the solar system's oldest solids: Astrophysical Journal, v. 675, p. 121-124, doi: $10.1086 / 533586$.

Connelly, J.N., and Bizzarro, M., 2009, Pb-Pb dating of chondrules from CV chondrites by progressive dissolution: Chemical Geology, v. 259, p. 143-151, doi:

10.1016/j.chemgeo.2008.11.003.

Connelly, J.N., Bizzarro, M., Krot, A.N., Nordlund, Å., Wielandt, D., and Ivanova, M.A., 2012, The absolute chronolgy and thermal processing of solids in the Solar protoplanetary disk: Science, v. 338, p. 651-655, doi: 10.1126/science.1226919.

Connolly, H.C.J., Desch, S.J., Ash, R.D., and Jones, R.H., 2006, Transient Heating 
Events in the Protoplanetary Nebula, in Lauretta, D.S. and McSween Jr., H.Y. eds., Meteorites and the Early Solar System II, Yuson, University of Arizona Press, p. 383-397.

Connolly, H.C., and Hewins, R.H., 1995, Chondrules as products of dust collisions with totally molten droplets within a dust-rich nebular environment: An experimental investigation: Geochimica et Cosmochimica Acta, v. 59, p. 3231-3246, doi: 10.1016/0016-7037(95)00207-G.

Connolly, H.C., and Hewins, R.H., 1991, The influence of bulk composition and dynamic melting conditions on olivine chondrule textures: Geochimica et Cosmochimica Acta, v. 55, doi: 10.1016/0016-7037(91)90459-I.

Connolly, H.C.J., and Jones, R.H., 2016, Chondrules: The canonical and noncanonical views: Journal of Geophysical Research: Planets, v. 121, p. 1885-1899, doi: 10.1002/ 2016JE005113.

Connolly, H.C., Jones, B.D., and Hewins, R.H., 1998, The flash melting of chondrules: An experimental investigation into the melting history and physical nature of chondrule precursors: Geochimica et Cosmochimica Acta, v. 62, p. 2725-2735, doi: 10.1016/S0016-7037(98)00176-8.

Connolly, H.C., and Love, S.G., 1998, The formation of chondrules: Petrologic tests of the shock wave model: Science, v. 280, p. 62-67, doi: 10.1126/science.280.5360.62.

Cuzzi, J.N., and Alexander, C.M.O., 2006, Chondrule formation in particle-rich nebular regions at least hundreds of kilometres across: Nature, v. 441, p. 483-485, doi: 10.1038/nature04834.

Dauphas, N., and Pourmand, A., 2011, Hf-W-Th evidence for rapid growth of Mars and its status as a planetary embryo: Nature, v. 473, p. 489-492, doi: 10.1038/nature 10077 .

Davison, T.M., Collins, G.S., and Bland, P.A., 2016, Mesoscale modeling of impact compaction of primitive solar system solids: Astrophysical Journal, v. 821, p. 1-17, doi: 10.3847/0004-637X/821/1/68.

Desch, S., 2006, How to make a chondrule: Nature, v. 441, p. 416-417.

Desch, S.J., Ciesla, F.J., Hood, L.L., and Nakamoto, T., 2005, Heating of chondritic materials in solar nebula shocks, in Chondrites and the Protoplanetary Disk, p. 849872.

Desch, S.J., and Connolly, H.C.J., 2002, A model of the thermal processing of particles in solar nebula shocks: Applications to the cooling rates of chondrules: Meteoritics and Planetary Science, v. 37, p. 183-207.

Desch, S.J., and Cuzzi, J.N., 2000, The generation of lightning in the solar nebula: Icarus, v. 143, p. 87-105, doi: 10.1006/icar.1999.6245.

Desch, S.J., Morris, M.A., Connolly, H.C., and Boss, A.P., 2010, A critical examination of the $\mathrm{x}$-wind model for chondrule and calcium-rich, aluminum-rich inclusion formation and radionuclide production: The Astrophysical Journal, v. 725, p. 692711, doi: 10.1088/0004-637X/725/1/692.

Desch, S.J., Morris, M.A., Connolly, H.C.J., and Boss, A.P., 2012, The importance of experiments: Constraints on chondrule formation models: Meteoritics and Planetary Science, v. 47, p. 1139-1156, doi: 10.1111/j.1945-5100.2012.01357.x. 
Desch, S.J., and Turner, N.J., 2015, High temperature ionization in protoplanetary disks: The Astrophysical Journal, v. 811, p. 1-16, doi: 10.1088/0004-637X/811/2/156.

Dodd, R.T., 1965, Preferred orientation of chondrules in chondrites: Icarus, v. 4, p. 308316, doi: 10.1016/0019-1035(65)90007-2.

Duggan, P., McBreen, B., Carr, A.J., Winston, E., Vaughan, G., Hanlon, L., McBreen, S., Metcalfe, L., Kvick, A., and Terry, A.E., 2003, Gamma-ray bursts and X-ray melting of material to form chondrules and planets: Astronomy and Astrophysics, v. 409, p. L9-L12, doi: 10.1051/0004-6361:20031238.

Dullemond, C.P., Harsono, D., Stammler, S.M., and Johansen, A., 2016, Forming chondrules in impact splashes. II. Volatile retention: Astrophysical Journal, v. 832, p. 1-19, doi: 10.3847/0004-637X/832/1/91.

Dullemond, C.P., Stammler, S.M., and Johansen, A., 2014, Forming chondrules in impact splashes. I. radiative cooling model: The Astrophysical Journal, v. 794, p. 1-12, doi: 10.1088/0004-637X/794/1/91.

Durinck, J., Legris, A., and Cordier, P., 2005, Pressure sensitivity of olivine slip systems: First-principle calculations of generalised stacking faults: Physics and Chemistry of Minerals, v. 32, p. 646-654, doi: 10.1007/s00269-005-0041-2.

Dzyurkevich, N., Turner, N.J., Henning, T., and Kley, W., 2013, Magnetized accretion and dead zones in protostellar disks: Astrophysical Journal, v. 765, p. 1-27, doi: 10.1088/0004-637X/765/2/114.

Ebert, S., and Bischoff, A., 2016, Genetic relationship between Na-rich chondrules and $\mathrm{Ca}, \mathrm{Al}-$ rich inclusions? - Formation of Na-rich chondrules by melting of refractory and volatile precursors in the solar nebula: Geochimica et Cosmochimica Acta, v. 177, p. 182-204, doi: 10.1016/j.gca.2016.01.014.

Eisenhour, D.D., Daulton, T.L., and Buseck, P.R., 1994, Electromagnetic Heating in the Early Solar Nebula and the Formation of Chondrules: Science, v. 265, p. 10671070.

Fedkin, A. V., Grossman, L., Ciesla, F.J., and Simon, S.B., 2012, Mineralogical and isotopic constraints on chondrule formation from shock wave thermal histories: Geochimica et Cosmochimica Acta, v. 87, p. 81-116, doi: 10.1016/j.gca.2012.03.020.

Fedkin, A. V., Grossman, L., Humayun, M., Simon, S.B., and Campbell, A.J., 2015, Condensates from vapor made by impacts between metal-, silicate-rich bodies: Comparison with metal and chondrules in CB chondrites: Geochimica et Cosmochimica Acta, v. 164, p. 236-261, doi: 10.1016/j.gca.2015.05.022.

Forman, L. V., Bland, P.A., Timms, N.E., Collins, G.S., Davison, T.M., Ciesla, F.J., Benedix, G.K., Daly, L., Trimby, P.W., Yang, L., and Ringer, S.P., 2016, Hidden secrets of deformation: Impact-induced compaction within a CV chondrite: Earth and Planetary Science Letters, v. 452, p. 133-145, doi: 10.1016/j.epsl.2016.07.050.

Forman, L. V., Bland, P.A., Timms, N.E., Daly, L., Benedix, G.K., Trimby, P.W., Collins, G.S., and Davison, T.M., 2017, Defining the mechanism for compaction of the CV chondrite parent body: Geology, v. 45, p. 559-562, doi: 10.1130/G38864.1.

Fossen, H., 2016, Structural Geology: Cambridge, Cambridge University Press, 235-251 p. 
Fox, G.E., and Hewins, R.H., 2005, Chondrule reheating experiments and relict olivine: Geochimica et Cosmochimica Acta, v. 69, p. 2441-2449, doi: 10.1016/j.gca.2004.11.014.

Friedrich, J.M., Rubin, A.E., Beard, S.P., Swindle, T.D., Isachsen, C.E., Rivers, M.L., and Macke, R.J., 2014, Ancient porosity preserved in ordinary chondrites: Examining shock and compaction on young asteroids: Meteoritics and Planetary Science, v. 49, p. 1214-1231, doi: 10.1111/maps.12328.

Friedrich, J.M., Ruzicka, A., Macke, R.J., Thostenson, J.O., Rudolph, R.A., Rivers, M.L., and Ebel, D.S., 2017, Relationships among physical properties as indicators of high temperature deformation or post-shock thermal annealing in ordinary chondrites: Geochimica et Cosmochimica Acta, v. 203, p. 157-174, doi: 10.1016/j.gca.2016.12.039.

Friedrich, J.M., Ruzicka, A., Rivers, M.L., Ebel, D.S., Thostenson, J.O., and Rudolph, R.A., 2013, Metal veins in the Kernouvé (H6 S1) chondrite: Evidence for pre- or syn-metamorphic shear deformation: Geochimica et Cosmochimica Acta, v. 116, p. 71-83, doi: 10.1016/j.gca.2013.01.009.

Fritz, J., Greshake, A., and Fernandes, V.A., 2017, Revising the shock classification of meteorites: Meteoritics and Planetary Science, v. 52, p. 1216-1232, doi: 10.1111/maps.12845.

Fujii, N., and Miyamoto, M., 1983, Constraints on the heating and cooling processes of chondrule formation, in Lunar and Planetary Science Conference, p. 19.

Gaboriaud, R.J., Darot, M., Gueguen, Y., and Woirgard, J., 1981, Dislocations in olivine indented at low temperatures: Physics and Chemistry of Minerals, v. 7, p. 100-104, doi: 10.1007/BF00309460.

Gattacceca, J., Bouvier, A., Grossman, J., Metzler, K., and Uehara, M., 2019, The Meteoritical Bulletin, No. 106: Meteoritics and Planetary Science, v. 54, p. 469-471, doi: 10.1111/maps.13215.

Gibbard, S.G., Levy, E.H., and Morfill, G.E., 1997, On the possibility of lightning in the protoplanetary nebula: Icarus, v. 130, p. 517-533.

Gooding, J.L., and Keil, K., 1981, Relative abundances of chondrule primary textural types in ordinary chondrites and their bearing on conditions of chondrule formation: Meteoritics, v. 16, p. 17-43, doi: 10.1111/j.1945-5100.1981.tb00183.x.

Grady, M.M., 2000, Catalogue of Meteorites: London, Natural History Museum, 696 p.

Grossman, J.N., and Brearley, A.J., 2005, The onset of metamorphism in ordinary and carbonaceous chondrites: Meteoritics and Planetary Science, v. 40, p. 87-122, doi: 10.1111/j.1945-5100.2005.tb00366.x.

Hanna, R.D., Ketcham, R.A., Zolensky, M., and Behr, W.M., 2015, Impact-induced brittle deformation, porosity loss, and aqueous alteration in the Murchison CM chondrite: Geochimica et Cosmochimica Acta, v. 171, p. 256-282, doi: 10.1016/j.gca.2015.09.005.

Hasegawa, Y., Wakita, S., Matsumoto, Y., and Oshino, S., 2016, Chondrule formation via impact jetting triggered by planetary accretion: Astrophysical Journal, v. 816, p. 1-14, doi: 10.3847/0004-637X/816/1/8.

Hevey, P.J., and Sanders, I.S., 2006, A model for planetisemal meltdown by $26 \mathrm{Al}$ and its 
implications for meteorite parent bodies: Meteoritics and Planetary Science, v. 41, p. 95-106.

Hewins, R.H., 1997, Chondrules: Annual Review of Earth and Planetary Sciences, v. 25, p. 61-83, doi: 10.1146/annurev.earth.25.1.61.

Hewins, R.H., 1983, Dynamic crystallization experiments as constraints on chondrule genesis, in King, E.A. ed., Chondrules and their Origins, Houston, Lunar and Planetary Institute, p. 122-133.

Hewins, R.H., 1991, Retention of sodium during chondrule melting: Geochimica et Cosmochimica Acta, v. 55, p. 935-942, doi: 10.1016/0016-7037(91)90152-U.

Hewins, R.H., Condie, C., Morris, M., Richardson, M.L.A., Ouellette, N., and Metcalf, M., 2018, Thermal History of CBb Chondrules and Cooling Rate Distributions of Ejecta Plumes: Astrophysical Journal, v. 855, p. 1-7, doi: 10.3847/20418213/aab15b.

Hewins, R.H., Connolly, H.C., and Libourel, G., 2005, Experimental Constraints on Chondrule Formation, in Chondrites and the Protoplanetary Disk, p. 286-316.

Hewins, R.H., and Radomsky, P.M., 1990, Temperature conditions for chondrule formation: Meteoritics, v. 25, p. 309-318, doi: 10.1111/j.1945-5100.1990.tb00715.x.

Hewins, R.H., Zanda, B., and Bendersky, C., 2012, Evaporation and recondensation of sodium in Semarkona type II chondrules: Geochimica et Cosmochimica Acta, v. 78, p. 1-17, doi: 10.1016/j.gca.2011.11.027.

Hezel, D.C., and Palme, H., 2008, Constraints for chondrule formation from Ca-Al distribution in carbonaceous chondrites: Earth and Planetary Science Letters, v. 265, p. 716-725, doi: 10.1016/j.epsl.2007.11.003.

Hezel, D.C., and Palme, H., 2010, The chemical relationship between chondrules and matrix and the chondrule matrix complementarity: Earth and Planetary Science Letters, v. 294, p. 85-93, doi: 10.1016/j.epsl.2010.03.008.

Holmén, B.A., and Wood, J.A., 1986, Chondrules that indent one another: Evidence for hot accretion? Abstracts and Program for the 49th Annual Meeting of the Meteoritical Society, p. H-2.

Hood, L.L., 1998, Thermal processing of chondrule precursors in planetesimal bow shocks: Meteoritics and Planetary Science, v. 33, p. 97-107, doi: 10.1111/j.19455100.1998.tb01611.x.

Hood, L.L., and Ciesla, F.J., 2001, The scale size of chondrule formation regions: Constraints imposed by chondrule cooling rates: Meteoritics and Planetary Science, v. 36, p. 1571-1585, doi: 10.1111/j.1945-5100.2001.tb01849.x.

Hood, L.L., Ciesla, F.J., Artemieva, N.A., Marzari, F., and Weidenschilling, S.J., 2009, Nebular shock waves generated by planetesimals passing through Jovian resonance: Possible sites for chondrule formation: Meteoritics and Planetary Science, v. 44, p. 327-342.

Hood, L.L., Ciesla, F.J., and Weidenschilling, S.J., 2005, Chondrule formation in planetesimal bow shocks: Heating and cooling rates, in Krot, A.N., Scott, E.R.D., and Reipurth, B. eds., Chondrites and the Protoplanetary Disk, p. 873-882.

Hood, L.L., and Horányi, M., 1991, Gas dynamic heating of chondrule precursor grains in the solar nebula: Icarus, v. 93, p. 259-269. 
Hood, L.L., and Weidenschilling, S.J., 2012, The planetesimal bow shock model for chondrule formation: A more quantitative assessment of the standard (fixed Jupiter) case: Meteoritics and Planetary Science, v. 47, p. 1715-1727, doi: 10.1111/maps.12006.

Horányi, M., and Robertson, S., 1996, Chondrule formation in lightning discharges: Status of theory and experiments, in Hewins, R.H., Jones, R.H., and Scott, E.R.D. eds., Chondrites and the Protoplanetary Disk, Cambridge, Cambridge University Press, p. 303-310.

Houseknecht, D.W., 1987, Assessing the elative importance of compaction processes and cementation to reduction of porosity in sandstones: American Association of Petroleum Geologists Bulletin, v. 71, p. 633-642.

Howard, E., 1802, Experiments and observations on certain stony and metalline substances, which at different times are said to have fallen on the Earth; Also on various kinds of native iron: Philosophical Transactions of the Royal Society of London, v. 92, p. 168-212, doi: 10.1098/rstl.1802.0009.

Hubbard, A., McNally, C.P., and Mac Low, M.-M., 2012, Short circuits in thermally ionized plasmas: A mechanism for intermittent heating of protoplanetary disks: Astrophysical Journal, v. 761, doi: 10.1088/0004-637X/761/1/58.

Hubbard, A., McNally, C.P., Mac Low, M.M., Ebel, D.S., and D’Alessio, P., 2013, The short circuit instability in protoplanetary disks: Processing high temperature minerals, in EPJ Web of Conferences, v. 46, doi: 10.1051/epjconf/20134604005.

Hugo, R.C., Ruzicka, A.M., and Rubin, A.E., 2019, Mesoscale and microscale shock effects in the LL6 S4 chondrites Saint-Séverin and Elbert: A tale of two breccias: Meteoritics and Planetary Science, v. 54, p. 1-21, doi: 10.1111/maps.13304.

Humayun, M., 2012, Chondrule cooling rates inferred from diffusive profiles in metal lumps from the Acfer 097 CR2 chondrite: Meteoritics and Planetary Science, v. 47, p. 1191-1208, doi: 10.1111/j.1945-5100.2012.01371.x.

Huss, G.R., Rubin, A.E., and Grossman, J.N., 2006, Thermal Metamorphism in Chondrites, in Lauretta, D.S. and McSween, H.Y.J. eds., Meteorites and the early solar system II, Tucson, University of Arizona Press, p. 567-586.

Hutcheon, I.D., Marhas, K.K., Krot, A.N., Goswami, J.N., and Jones, R.H., 2009, 26Al in plagioclase-rich chondrules in carbonaceous chondrites: Evidence for an extended duration of chondrule formation: Geochimica et Cosmochimica Acta, v. 73, p. 5080-5099, doi: 10.1016/j.gca.2009.04.042.

Hutchison, R., 1996a, Chondrules and their associates in ordinary chondrites: A planetary connection?, in Hewins, R.H., Jones, R.H., and E.R.D., S. eds., Chondrules and the Protoplanetary Disk, Cambridge, Cambridge University Press, p. 311-318.

Hutchison, R., 1996b, Hot accretion of ordinary chondrites : The rocks don't lie: Lunar and Planetary Institute, v. 27, p. 579-580.

Hutchison, R., Alexander, C.M.O., and Bridges, J.C., 1998, Elemental redistribution in Tieschitz and the origin of white matrix: Meteoritics and Planetary Science, v. 33, p. 1169-1179, doi: 10.1111/j.1945-5100.1998.tb01721.x.

Hutchison, R., and Bevan, A.W.R., 1983, Conditions and Time of Chondrule Accretion, in Chondrules and their Origins, p. 162-179. 
Hutchison, R., Bevan, A.W.R., Agrell, S.O., and Ashworth, J.R., 1979, Accretion temperature of the Tieschitz, H3, chondritic meteorite: Nature, v. 280, p. 116-119, doi: $10.1038 / 280116 \mathrm{a} 0$.

Hutchison, R., Bridges, J.C., and Gilmour, J.D., 2005, Chondrules: Chemical, petrographic and chronologic clues to their origin by impact, in Chondrites and the Protoplanetary Disk, p. 933-950.

Hutson, M.L., Ruzicka, A.M., and Nazari, M., 2014, Diverse and unusual O-chondrites from the Lut Desert, Iran, in 77th Annual Meteoritical Society Meeting, p. 5180, doi: 10.1016/j.molstruc.2014.05.049.

Jacquet, E., 2014, The quasi-universality of chondrule size as a constraint for chondrule formation models: Icarus, v. 232, p. 176-186, doi: 10.1016/j.icarus.2014.01.012.

Jacquet, E., and Thompson, C., 2014, Chondrule destruction in nebular shocks: Astrophysical Journal, v. 797, p. 1-16, doi: 10.1088/0004-637X/797/1/30.

Jamsja, N., and Ruzicka, A., 2010, Shock and thermal history of Northwest Africa 4859, an annealed impact-melt breccia of LL chondrite parentage containing unusual igneous features and pentlandite: Meteoritics and Planetary Science, v. 45, p. 828849, doi: 10.1111/j.1945-5100.2010.01056.x.

Johansen, A., and Okuzumi, S., 2018, Harvesting the decay energy of 26Al to drive lightning discharge in protoplanetary discs: Astronomy and Astrophysics, v. 609, p. 1-22, doi: 10.1051/0004-6361/201630047.

Johnson, B.C., Minton, D.A., Melosh, H.J., and Zuber, M.T., 2015, Impact jetting as the origin of chondrules: Nature, v. 517, p. 339-341, doi: 10.1038/nature14105.

Jones, R.H., Lee, T., Connolly, H.C.J., Love, S.G., and Shang, H., 2000, Formation of Chondrules and CAIs: Theory VS. Observation, in Protostars and planets IV, p. 927-962.

Jones, R.H., and Lofgren, G.E., 1993, A comparison of FeO-rich, porphyritic olivine chondrules in unequilibrated chondrites and experimental analogues: Meteoritics, v. 28, p. 213-221, doi: 10.1111/j.1945-5100.1993.tb00759.x.

Joreau, P., Leroux, H., and Doukhan, J.C., 1997, A transmission electron microscope investigation of shock metamorphism in olivine of the Ilafegh 013 chondrite: Meteoritics and Planetary Science, v. 32, p. 309-316, doi: 10.1111/j.19455100.1997.tb01268.x.

Joung, M.K.R., Mac Low, M., and Ebel, D.S., 2004, Chondrule formation and protoplanetary disk heating by current sheets in nonideal magnetohydrodynamic turbulence: Astrophysical Journal, v. 606, p. 532-541, doi: 10.1086/381651.

Jung, H., Katayama, I., Jiang, Z., Hiraga, T., and Karato, S., 2006, Effect of water and stress on the lattice-preferred orientation of olivine: Tectonophysics, v. 421, p. 1-22, doi: 10.1016/j.tecto.2006.02.011.

Karato, S., Jung, H., Katayama, I., and Skemer, P., 2008, Geodynamic significance of seismic anisotropy of the upper mantle: new insights from laboratory studies: Annual Review of Earth and Planetary Sciences, v. 36, p. 59-95, doi: 10.1146/annurev.earth.36.031207.124120.

Kato, T., Nakamoto, T., and Miura, H., 2006, Maximal size of chondrules in shock wave heating model: Stripping of liquid surface in a hypersonic rarefied gas flow: 
Meteoritics and Planetary Science, v. 41, p. 49-65, doi: 10.1111/j.19455100.2006.tb00192.x.

Kennedy, A.K., Hutchison, R., Hutcheon, I.D., and Agrell, S.O., 1992, A unique high $\mathrm{Mn} / \mathrm{Fe}$ microgabbro in the Parnallee (LL3) ordinary chondrite: Nebular mixture or planetary differentiate from a previously unrecognized planetary body? Earth and Planetary Science Letters, v. 113, p. 191-205, doi: 10.1016/0012-821X(92)90219-L.

Kirby, S.H., and Wegner, M.W., 1978, Dislocation substructure of mantle-derived olivine as revealed by selective chemical etching and transmission electron microscopy: Physics and Chemistry of Minerals, v. 3, p. 309-330, doi: 10.1007/BF00311845.

Kita, N.T., Huss, G.R., Tachibana, S., Amelin, Y., Nyquist, L.E., and Hutcheon, I.D., 2005, Constraints on the origin of ohondrules and CAIs from short-lived and longLived radionuclides, in Krot, A.N., Scott, E.R.D., and Reipurth, B. eds., Chondrites and the Protoplanetary Disk, p. 558-587.

Kita, N.T., Nagahara, H., Togashi, S., and Morishita, Y., 2000, A short duration of chondrule formation in the solar nebula: Evidence from 26Al in Semarkona ferromagnesian chondrules: Geochimica et Cosmochimica Acta, v. 64, p. 39133922, doi: 10.1016/S0016-7037(00)00488-9.

Kita, N.T., and Ushikubo, T., 2012, Evolution of protoplanetary disk inferred from 26Al chronology of individual chondrules: Meteoritics and Planetary Science, v. 47, p. 1108-1119, doi: 10.1111/j.1945-5100.2011.01264.x.

Klein, C., and Dutrow, B., 2007, The Manual of Mineral Science: John Wiley \& Sons, $675 \mathrm{p}$.

Kleine, T., Mezger, K., Palme, H., Scherer, E., and Münker, C., 2005, Early core formation in asteroids and late accretion of chondrite parent bodies: Evidence from 182Hf-182W in CAIs, metal-rich chondrites, and iron meteorites: Geochimica et Cosmochimica Acta, v. 69, p. 5805-5818, doi: 10.1016/j.gca.2005.07.012.

de Kloe, R., Drury, M., and Farrer, J.K., 2002, Determination of activated slip systems in experimentally deformed olivine-orthopyroxene polycrystals using EBSD: Microscopy and Microanalysis, v. 8, p. 680-681.

Kracher, A., Scott, E.R.D., and Keil, K., 1984, Relict and other anomalous grains in chondrules: Implications for chondrule formation, in Lunar and Planetary Science Conference XIV, p. 559-566.

Krot, A.N., Amelin, Y., Bland, P., Ciesla, F.J., Connelly, J., Davis, A.M., Huss, G.R., Hutcheon, I.D., Makide, K., Nagashima, K., Nyquist, L.E., Russell, S.S., Scott, E.R.D., Thrane, K., et al., 2009, Origin and chronology of chondritic components: A review: Geochimica et Cosmochimica Acta, v. 73, p. 4963-4997, doi: 10.1016/j.gca.2008.09.039.

Krot, A.N., Amelin, Y., Cassen, P., and Meibom, A., 2005, Young chondrules in CB chondrites from a giant impact in the early Solar System: Nature, v. 436, p. 989992, doi: 10.1038/nature03830.

Krot, A.N., Ivanova, M.A., and Ulyanov, A.A., 2007, Chondrules in the CB/CH-like carbonaceous chondrite Isheyevo: Evidence for various chondrule-forming mechanisms and multiple chondrule generations: Chemie der Erde, v. 67, p. 283300, doi: 10.1016/j.chemer.2006.04.001. 
Krot, A.N., Meibom, A., Russell, S.S., Alexander, C.M.O., Jeffries, T.E., and Keil, K., 2001, A new astrophysical setting for chondrule formation: Science, v. 291, p. 1776-1779, doi: 10.1126/science.291.5509.1776.

Krot, A.N., and Nagashima, K., 2017, Constraints on mechanisms of chondrule formation from chondrule precursors and chronology of transient heating events in the protoplanetary disk: Geochemical Journal, v. 51, p. 45-68, doi: 10.2343/geochemj.2.0459.

Kruijer, T.S., Burkhardt, C., Budde, G., and Kleine, T., 2017, Age of Jupiter inferred from the distinct genetics and formation times of meteorites: Proceedings of the National Academy of Sciences, v. 114, p. 6712-6716, doi: 10.1073/pnas.1704461114.

Langenhorst, F., Joreau, P., and Doukhan, J.C., 1995, Thermal and shock metamorphism of the Tenham chondrite: A TEM examination: Geochimica et Cosmochimica Acta, v. 59, p. 1835-1845, doi: 10.1016/0016-7037(95)00086-F.

Larson, R.B., 2002, The role of tidal interactions in star formation: Monthly Notices of the Royal Astronomical Society, v. 332, p. 155-164, doi: 10.1046/j.13658711.2002.05281.x.

Lauretta, D.S., Nagahara, H., and Alexander, M.O., 2006, Petrology and the origin of ferromagnesian silicate chondrules, in Lauretta, D.S. and Mcsween, H.Y.J. eds., Meteorites and the Early Solar System II, Tucson, University of Arizona Press, p. 431-459.

Leroux, H., 2001, Microstructural shock signatures of major minerals in meteorites: European Journal of Mineralogy, v. 13, p. 253-272, doi: 10.1127/09351221/01/0013-0253.

Leroux, H., Doukhan, J.C., and Guyot, F., 1996, An analytical electron microscopy (AEM) investigation of opaque inclusions in some type 6 ordinary chondrites: Meteoritics and Planetary Science, v. 31, p. 767-776, doi: 10.1111/j.19455100.1996.tb02111.x.

Lewis, R.D., Lofgren, G.E., Franzen, H.F., and Windom, K.E., 1993, The effect of Na vapor on the Na content of chondrules: Meteoritics, v. 28, p. 622-628, doi: 10.1111/j.1945-5100.1993.tb00632.x.

Libourel, G., and Chaussidon, M., 2011, Oxygen isotopic constraints on the origin of Mgrich olivines from chondritic meteorites: Earth and Planetary Science Letters, v. 301, p. 9-21, doi: 10.1016/j.eps1.2010.11.009.

Libourel, G., and Krot, A.N., 2007, Evidence for the presence of planetesimal material among the precursors of magnesian chondrules of nebular origin: Earth and Planetary Science Letters, v. 254, p. 1-8, doi: 10.1016/j.epsl.2006.11.013.

Lichtenberg, T., Golabek, G.J., Dullemond, C.P., Schönbächler, M., Gerya, T. V., and Meyer, M.R., 2018, Impact splash chondrule formation during planetesimal recycling: Icarus, v. 302, p. 27-43, doi: 10.1016/j.icarus.2017.11.004.

Lichtenburg, T., Golabek, G.J., Dullemond, C.P., Schönbächler, M., Gerya, T.V., and Meyer, M.R., 2017, Impact splash chondrule formation revisited, in European Planetary Science Congress, v. 11, p. 6-7.

Lloyd, G.E., Farmer, A.B., and Mainprice, D., 1997, Misorientation analysis and the 
formation and orientation of subgrain and grain boundaries: Tectonophysics, v. 279, p. 55-78, doi: 10.1016/S0040-1951(97)00115-7.

Lofgren, G.E., 1996, A dynamic crystallization model for chondrule melts, in Hewins, R.H., Jones, R.H., and Scott, E.R.D. eds., Chondrules and the Protoplanetary Disk, Cambridge, Cambridge University Press, p. 187-196.

Lofgren, G., and Russell, W.J., 1986, Dynamic crystallization of chondrule melts of porphyritic and radial pyroxene composition: Geochimica et Cosmochimica Acta, v. 50, p. 1715-1726, doi: 10.1016/0016-7037(86)90133-X.

Lugmair, G.W., and Shukolyukov, A., 2001, Early solar system events and timescales: Meteoritics and Planetary Science, v. 36, p. 1017-1026.

Mann, C.R., Boley, A.C., and Morris, M.A., 2016, Planetary embryo bow shocks as a mechanism for chondrule formation: Astrophysical Journal, v. 818, p. 1-20, doi: 10.3847/0004-637X/818/2/103.

Marzari, F., and Weidenschilling, S., 2002, Mean motion resonances, gas drag, and supersonic planetesimals in the solar nebula: Celestial Mechanics and Dynamical Astronomy, v. 82, p. 225-242, doi: 10.1023/A:1015067000812.

McBreen, B., and Hanlon, L., 1999, Gamma-ray bursts and the origin of chondrules and planets: Astronomy and Astrophysics, v. 351, p. 759-765.

McBreen, B., Winston, E., McBreen, S., and Hanlon, L., 2005, Gamma-ray bursts and other sources of giant lightning discharges in protoplanetary systems: Astronomy and Astrophysics, v. 429, p. L41-L45, doi: 10.1051/0004-6361:200400102.

McCoy, T.J., Keil, K., Scott, E.R.D., Benedix, G.K., Ehlmann, A.J., Mayeda, T.K., and Clayton, R.N., 1994, Low-FeO Ordinary Chondrites: A Nebular Origin and New Chondrite Parent Body: Abstracts of the 25th Lunar and Planetary Science Conference, p. 865.

McSween, H.Y.J., 1977, Chemical and petrographic constraints on the origin of chondrules and inclusions in carbonaceous chondrites: Geochimica et Cosmochimica Acta, v. 41, doi: 10.1016/0016-7037(77)90216-2.

McSween, H.Y.J., Sears, D.W., and Dodd, R.T., 1988, Thermal Metamorphism, in Kerridge, J.F. and Matthews, M.S. eds., Meteorites and the Early Solar System, Tuscon, University of Arizona Press, p. 102-113.

Metzler, K., 2012, Ultrarapid chondrite formation by hot chondrule accretion? Evidence from unequilibrated ordinary chondrites: Meteoritics and Planetary Science, v. 47, p. 2193-2217, doi: 10.1111/maps.12009.

Metzler, K., and Pack, A., 2016, Chemistry and oxygen isotopic composition of cluster chondrite clasts and their components in LL3 chondrites: Meteoritics and Planetary Science, v. 51, p. 276-302, doi: 10.1111/maps.12592.

Michel-Levy, M.C., and Lautie, A., 1981, Microanalysis by Raman spectroscopy of carbon in the Tieschitz chondrite: Nature, v. 292, p. 321-322, doi: $10.1038 / 292321 \mathrm{a} 0$.

Miura, H., and Nakamoto, T., 2005, A shock-wave heating model for chondrule formation II. Minimum size of chondrule precursors: Icarus, v. 175, p. 289-304, doi: 10.1016/j.icarus.2004.11.011.

Miura, H., and Nakamoto, T., 2007, Shock-wave heating model for chondrule formation: 
Hydrodynamic simulation of molten droplets exposed to gas flows: Icarus, v. 188, p. 246-265, doi: 10.1016/j.icarus.2006.11.008.

Miura, H., and Nakamoto, T., 2006, Shock-wave heating model for chondrule formation: Prevention of isotopic fractionation: The Astrophysical Journal, v. 651, p. 12721295, doi: 10.1086/507402.

Miura, H., Nakamoto, T., and Susa, H., 2002, A shock-wave heating model for chondrule formation: Effects of evaporation and gas flows on silicate particles: Icarus, v. 160, p. 258-270, doi: 10.1006/icar.2002.6964.

Miura, H., and Yamamoto, T., 2014, A new estimate of the chondrule cooling rate deduced from an analysis of compositional zoning of relict olivine: Astronomical Journal, v. 147, p. 1-9, doi: 10.1088/0004-6256/147/3/54.

Miyamoto, M., Mikouchi, T., and Jones, R.H., 2009, Cooling rates of porphyritic olivine chondrules in the Semarkona (LL3.00) ordinary chondrite: A model for diffusional equilibration of olivine during fractional crystallization: Meteoritics and Planetary Science, v. 44, p. 521-530, doi: 10.1111/j.1945-5100.2009.tb00748.x.

Morris, M.A., Boley, A.C., Desch, S.J., and Athanassiadou, T., 2012, Chondrule formation in bow shocks around eccentric planetary embryos: Astrophysical Journal, v. 752, p. 1-17, doi: 10.1088/0004-637X/752/1/27.

Morris, M.A., and Desch, S.J., 2010, Thermal histories of chondrules in solar nebula shocks: Astronomical Journal, v. 722, p. 1474-1494, doi: 10.1088/0004637X/722/2/1474.

Morris, M.A., Weidenschilling, S.J., and Desch, S.J., 2016, The effect of multiple particle sizes on cooling rates of chondrules produced in large-scale shocks in the solar nebula: Meteoritics and Planetary Science, v. 51, p. 870-883, doi:

10.1111/maps.12631-2309.

Müller, W.F., Weinbruch, S., Walter, R., and Müller-Beneke, G., 1995, Transmission electron microscopy of chondrule minerals in the Allende meteorite: Constraints on the thermal and deformational history of granular olivine-pyroxene chondrules: Planetary and Space Science, v. 43, p. 469-483, doi: 10.1016/0032-0633(94)00181P.

Müller, W.F., and Wlotzka, F., 1982, Mineralogical study of the Leoville meteorite (CV3): macroscopic texture and transmission electron microscopic observations, in Lunar and Planetary Institute Science Conference Abstracts, p. 558-559.

Muranushi, T., 2010, Dust-dust collisional charging and lightning in protoplanetary discs: Monthly Notices of the Royal Astronomical Society, v. 401, p. 2641-2664, doi: 10.1111/j.1365-2966.2009.15848.x.

Muranushi, T., Okuzumi, S., and Inutsuka, S., 2012, Interdependence of electric discharge and magnetorotational instability in protoplanetary disks: Astrophysical Journal, v. 760, p. 1-14, doi: 10.1088/0004-637X/760/1/56.

Nagasawa, M., Tanaka, K.K., Tanaka, H., Nakamoto, T., Miura, H., and Yamamoto, T., 2014, Revisiting Jovian-resonance induced chondrule formation: Astrophysical Journal Letters, v. 794, p. 1-5, doi: 10.1088/2041-8205/794/1/L7.

Nagashima, K., Moriuchi, Y., Tsukamoto, K., Tanaka, K.K., and Kobatake, H., 2008, Critical cooling rates for glass formation in levitated $\mathrm{Mg} 2 \mathrm{SiO} 4-\mathrm{MgSiO} 3$ chondrule 
melts: Journal of Mineralogical and Petrological Sciences, v. 103, p. 204-208, doi: 10.2465/jmps.070620c.

Nakamoto, T., Hayashi, M.R., Kita, N.T., and Tachibana, S., 2005, Chondrule-forming shock waves in the solar nebula by x-ray flares, in Krot, A.N., Scott, E.R.D., and Reipurth, B. eds., Chondrites and the Protoplanetary Disk, p. 883-892.

Nakamura, T., Tomeoka, K., Sekine, T., and Takeda, H., 1995, Impact-induced chondrule flattening in the Allende CV3 carbonaceous chondrite: Shock experiments: Meteoritics, v. 30, p. 344-347, doi: 10.1111/j.1945-5100.1995.tb01133.x.

Nakamura, T., Tomeoka, K., and Takeda, H., 1992, Shock effects of the Leoville CV carbonaceous chondrite: A transmission electron microscope study: Earth and Planetary Science Letters, v. 114, p. 159-170, doi: 10.1016/0012-821X(92)90158-R.

Oulton, J., Humayun, M., Fedkin, A., and Grossman, L., 2016, Chemical evidence for differentiation, evaporation and recondensation from silicate clasts in Gujba: Geochimica et Cosmochimica Acta, v. 177, p. 254-274, doi: 10.1016/j.gca.2016.01.008.

Palme, H., Hezel, D.C., and Ebel, D.S., 2015, The origin of chondrules: Constraints from matrix composition and matrix-chondrule complementarity: Earth and Planetary Science Letters, v. 411, p. 11-19, doi: 10.1016/j.epsl.2014.11.033.

Palme, H., Spettel, B., Kurat, G., and Zinner, E., 1992, Origin of Allende chondrules, in Lunar and Planetary Science Conference XXIII, p. 1021-1022.

Passchier, C.W., and Trouw, R.A.J., 2005, Microtectonics: Heidelberg, Springer-Verlag Berlin Heidelberg, 31-37 p.

Pilipp, W., Hartquist, T.W., Morfill, G.E., and Levy, E.H., 1998, Chondrule formation by lightning in the Protosolar Nebula? Astronomy and Astrophysics, v. 331, p. 121146.

Poppe, T., Güttler, C., and Springborn, T., 2010, Thermal metamorphoses of cosmic dust aggregates: Experiments by furnace, electrical gas discharge, and radiative heating: Earth, Planets and Space, v. 62, p. 53-56, doi: 10.5047/eps.2008.10.001.

Prior, D.J., Boyle, A.P., Brenker, F., Cheadle, M.C., Austin, D., Lopez, G., Peruzzo, L., Potts, G.J., Reddy, S., Spiess, R., Timms, N.E., Trimby, P., Wheeler, J., and Zetterström, L., 1999, The application of electron backscatter diffraction and orientation contrast imaging in the SEM to textural problems in rocks: American Mineralogist, v. 84, p. 1741-1759, doi: 10.2138/am-1999-11-1204.

Radomsky, P.M., and Hewins, R.H., 1990, Formation conditions of pyroxene-olivine and magnesian olivine chondrules: Geochimica et Cosmochimica Acta, v. 54, p. 34753490, doi: 10.1016/0016-7037(90)90299-Z.

Raleigh, C.B., 1965, Glide mechanisms in experimentally deformed minerals: Science, v. 150, p. 739-741, doi: 10.1126/science.150.3697.739.

Raleigh, C.B., 1968, Mechanisms of plastic deformation of olivine: Journal of Geophysical Research, v. 73, p. 5391-5406, doi: 10.1029/JB073i016p05391.

Raleigh, C.B., 1967, Plastic deformation of upper mantle silicate minerals: Geophysical Journal of the Royal Astronomical Society, v. 14, p. 45-49, doi: 10.1111/j.1365246X.1967.tb06220.x.

Raterron, P., Amiguet, E., Chen, J., Li, L., and Cordier, P., 2009, Experimental 
deformation of olivine single crystals at mantle pressures and temperatures: Physics of the Earth and Planetary Interiors, v. 172, p. 74-83, doi: 10.1016/j.pepi.2008.07.026.

Raterron, P., Chen, J., Li, L., Weidner, D., and Cordier, P., 2007, Pressure-induced slipsystem transition in forsterite: Single-crystal rheological properties at mantle pressure and temperature: American Mineralogist, v. 92, p. 1436-1445, doi: 10.2138/am.2007.2474.

Rubin, A.E., 1992, A shock-metamorphic model for silicate darkening and compositionally variable plagioclase in $\mathrm{CK}$ and ordinary chondrites: Geochimica et Cosmochimica Acta, v. 56, p. 1705-1714, doi: 10.1016/0016-7037(92)90236-C.

Rubin, A.E., 2003, Chromite-plagioclase assemblages as a new shock indicator; Implications for the shock and thermal histories of ordinary chondrites: Geochimica et Cosmochimica Acta, v. 67, p. 2695-2709, doi: 10.1016/S0016-7037(03)00107-8.

Rubin, A.E., 2013, Multiple melting in a four-layered barred-olivine chondrule with compositionally heterogeneous glass from LL3.0 Semarkona: Meteoritics and Planetary Science, v. 48, p. 445-456, doi: 10.1111/maps.12069.

Rubin, A.E., 1995a, Petrologic evidence for collisional heating of chondritic asteroids: Icarus, v. 113, p. 156-167, doi: 10.1006/icar.1995.1013.

Rubin, A.E., 2002, Post-shock annealing of Miller Range 99301 (LL6): Implications for impact heating of ordinary chondrites: Geochimica et Cosmochimica Acta, v. 66, p. 3327-3337, doi: 10.1016/S0016-7037(02)00916-X.

Rubin, A.E., 2004, Postshock annealing and postannealing shock in equilibrated ordinary chondrites: Implications for the thermal and shock histories of chondritic asteroids: Geochimica et Cosmochimica Acta, v. 68, p. 673-689, doi: 10.1016/S00167037(00)00452-6.

Rubin, A.E., 1995b, Pouring "cold water" on hot accretion: Meteoritics, v. 30, p. 568569.

Rubin, A.E., and Brearley, A.J., 1996, A critical evaluation of the evidence for hot accretion: Icarus, v. 124, p. 86-96, doi: 10.1006/icar.1996.0191.

Rubin, A.E., and Jones, R.H., 2003, Spade: An H chondrite impact-melt breccia that experienced post-shock annealing: Meteoritics and Planetary Science, v. 38, p. 1507-1520, doi: 10.1111/j.1945-5100.2003.tb00254.x.

Rubin, A.E., and Krot, A.N., 1996, Multiple heaitng of chondrules, in Hewins, R.H., Jones, R.H., and Scott, E.R.D. eds., Chondrules and the Protoplanetary Disk, Cambridge, Cambridge University Press, p. 173-180.

Rubin, A.E., Sailer, A.L., and Wasson, J.T., 1999, Troilite in the chondrules of type-3 ordinary chondrites: Implications for chondrule formation: Geochimica et Cosmochimica Acta, v. 63, p. 2281-2298, doi: 10.1016/S0016-7037(99)00119-2.

Rubin, A.E., and Swindle, T.D., 2011, Flattened chondrules in the LAP 04581 LL5 chondrite: Evidence for an oblique impact into LL3 material and subsequent collisional heating: Meteoritics and Planetary Science, v. 46, p. 587-600, doi: 10.1111/j.1945-5100.2011.01176.x.

Rudraswami, N.G., and Goswami, J.N., 2007, 26Al in chondrules from unequilibrated L chondrites: Onset and duration of chondrule formation in the early solar system: 
Earth and Planetary Science Letters, v. 257, p. 231-244, doi:

10.1016/j.eps1.2007.02.034.

Russell, S.S., Zolensky, M., Righter, K., Folco, L., Jones, R., Connolly, H.C., Grady, M.M., and Grossman, J.N., 2005, The Meteoritical Bulletin, No. 89, 2005

September: Meteoritics and Planetary Science, v. 40, p. A201-A263, doi: 10.1111/j.1945-5100.2005.tb00425.x.

Rutter, E.H., 1976, The kinetics of rock deformation by pressure solution: Philosophical Transactions of the Royal Society of London, v. 283, p. 203-219.

Ruzicka, A., 2012, Chondrule formation by repeated evaporative melting and condensation in collisional debris clouds around planetesimals: Meteoritics and Planetary Science, v. 47, p. 2218-2236, doi: 10.1111/j.1945-5100.2012.01412.x.

Ruzicka, A., Floss, C., and Hutson, M., 2008, Relict olivine grains, chondrule recycling, and implications for the chemical, thermal, and mechanical processing of nebular materials: Geochimica et Cosmochimica Acta, v. 72, p. 5530-5557, doi: 10.1016/j.gca.2008.08.017.

Ruzicka, A., Grossman, J., Bouvier, A., and Agee, C., 2014, The Meteoritical Bulletin, No. 103: Meteoritics \& Planetary Science, v. 52, p. 1014, doi: 10.1111/maps. 12888.

Ruzicka, A., Hiyagon, H., Hutson, M., and Floss, C., 2007, Relict olivine, chondrule recycling, and the evolution of nebular oxygen reservoirs: Earth and Planetary Science Letters, v. 257, p. 274-289, doi: 10.1016/j.eps1.2007.02.037.

Ruzicka, A.M., and Hugo, R.C., 2017, EBSD analyses of seven ordinary chondrites: Deformation metrics and implications for parent body thermal histories, in 80th Annual Meeting of the Meteoritical Society 2017,.

Ruzicka, A.M., and Hugo, R.C., 2018, Electron backscatter diffraction (EBSD) study of seven heavily metamorphosed chondrites: Deformation systematics and variations in pre-shock temperature and post-shock annealing: Geochimica et Cosmochimica Acta, v. 234, p. 115-147.

Ruzicka, A., Hugo, R., and Hutson, M., 2015, Deformation and thermal histories of ordinary chondrites: Evidence for post-deformation annealing and syn-metamorphic shock: Geochimica et Cosmochimica Acta, v. 163, p. 219-233, doi: 10.1016/j.gca.2015.04.030.

Ruzicka, A., Snyder, G.A., and Taylor, L.A., 2000, Crystal-bearing lunar spherules: Impact melting of the Moon's crust and implications for the origin of meteoritic chondrules: Meteoritics and Planetary Science, v. 35, p. 173-192, doi: 10.1111/j.1945-5100.2000.tb01984.x.

Ruzmaikina, T.V., and Ip, W.H., 1996, Chondrule formation in the accretion shock, in Hewins, R.H., Jones, R.H., and Scott, E.R.D. eds., Chondrules and the Protoplanetary Disk, Cambridge, Cambridge University Press, p. 277-284.

Sanders, I.S., and Hill, H.G.M., 1994, Multistage compound chondrules and molded chondrules in the Bovedy (L3) meteorite: Meteoritics, v. 29, p. 527-528.

Sanders, I.S., and Scott, E.R.D., 2012, The origin of chondrules and chondrites: Debris from low-velocity impacts between molten planetesimals? Meteoritics and Planetary Science, v. 47, p. 2170-2192, doi: 10.1111/maps.12002.

Sanders, I.J., and Taylor, G.J., 2005, Implications of 26Al in Nebular Dust: Formation of 
Chondrules by Disruption of Molten Planetesimals, in Chondrites and the Protoplanetary Disk, p. 915-932.

Scherstén, A., Elliott, T., Hawkesworth, C., Russell, S., and Masarik, J., 2006, Hf-W evidence for rapid differentiation of iron meteorite parent bodies: Earth and Planetary Science Letters, v. 241, p. 530-542, doi: 10.1016/j.epsl.2005.11.025.

Schmitt, R.T., 2000, Shock experiments with the H6 chondrite Kernouvé: Pressure calibration of microscopic shock effects: Meteoritics and Planetary Science, v. 35, p. 545-560, doi: 10.1111/j.1945-5100.2000.tb01435.x.

Schmitt, R.T., Deutsch, A., and Stöffler, D., 1994, Shock recovery experiments with the H6 chondrite Kernouvé at preshock temperatures of 293 and $920 \mathrm{~K}$, in Meteoritics, v. 29 , p. 529-530.

Van Schmus, W.R., and Wood, J.A., 1967, A chemical-petrologic classification for the chondritic meteorites: Geochimica et Cosmochimica Acta, v. 31, p. 747-765, doi: 10.1016/S0016-7037(67)80030-9.

Schultz, P.H., 1996, Shear heating by oblique impacts, in Geological Society of America, 28th annual meeting., p. 384.

Scott, E.R.D., 2007, Chondrites and the protoplanetary disk: Annual Review of Earth and Planetary Sciences, v. 35, p. 577-620, doi:

10.1146/annurev.earth.35.031306.140100.

Scott, E.R.D., Keil, K., and Stöffler, D., 1992, Shock metamorphism of carbonaceous chondrites: Geochimica et Cosmochimica Acta, v. 56, p. 4281-4293, doi: 10.1016/0016-7037(92)90268-N.

Scott, E.R.D., and Krot, A.N., 2005, Chondritic meteorites and the high-temperature nebular origins of their components, in Krot, A.N., Scott, E.R.., and Reipurth, B. eds., Chondrites and the Protoplanetary Disk, p. 15-53.

Sharp, T.G., and de Carli, P.S., 2006, Shock effects in meteorites, in Lauretta, D.S. and $\mathrm{McS}$ ween, H.Y.J. eds., Meteorites and the Early Solar System II, Tuscon, University of Arizona Press, p. 653-677.

Shu, F.H., Shang, H., Glassgold, A.E., and Lee, T., 1997, X-rays and fluctuating X-winds from protostars: Science, v. 277, p. 1475-1479, doi: 10.1126/science.277.5331.1475.

Shu, H.F., Shang, H., Gounelle, M., Glassgold, A.E., and Lee, T., 2001, The origin of chondrules and refractory inclusions in chondritic meteorites: Astronomical Journal, v. 548, p. 1029-1050.

Sirono, S., 2006, Can a chondrule precursor survive a shock wave? Astronomy and Astrophysics, v. 455, p. 379-384, doi: 10.1051/0004-6361:20065166.

Skinner, W.R., 1989a, Cold vs hot accretion of Tieschitz and other chondrites, in Lunar Planetary Science Conference XX, p. 1018-1019.

Skinner, W.R., 1989b, Compaction and lithification of chondrites, in Lunar and Planetary Science Conference XX, p. 1020-1021.

Sneyd, D.S., McSween, H.Y., Sugiura, N., Strangway, D.W., and Nord, G.L., 1988, Origin of petrofabrics and magnetic anisotropy in ordinary chondrites: Meteoritics, v. 23, p. 139-149, doi: 10.1111/j.1945-5100.1988.tb00909.x.

Sokol, A.K., Bischoff, A., Marhas, K.K., Mezger, K., and Zinner, E., 2007, Late 
accretion and lithification of chondritic parent bodies: $\mathrm{Mg}$ isotope studies on fragments from primitive chondrites and chondritic breccias: Meteoritics and Planetary Science, v. 42, p. 1291-1308, doi: 10.1111/j.1945-5100.2007.tb00575.x.

Sorby, H.C., 1877, On the structure and origin of meteorites: Nature, v. 15, p. 495-498, doi: $10.1038 / 015547 \mathrm{c} 0$.

Soulié, C., Libourel, G., and Tissandier, L., 2017, Olivine dissolution in molten silicates: An experimental study with application to chondrule formation: Meteoritics and Planetary Science, v. 52, p. 225-250, doi: 10.1111/maps.12792.

Stammler, S.M., and Dullemond, C.P., 2014, A critical analysis of shock models for chondrule formation: Icarus, v. 242, p. 1-10, doi: 10.1016/j.icarus.2014.07.024.

Stöffler, D., Bischoff, A., Buchwald, V., and Rubin, A.E., 1988, Shock effects in meteorites, in Kerridge, J.F. and Matthews, M.S. eds., Meteorites and the Early Solar System, Tucson, University of Arizona Press, p. 165-202.

Stöffler, D., Hamann, C., and Metzler, K., 2018, Shock metamorphism of planetary silicate rocks and sediments: Proposal for an updated classification system: Meteoritics and Planetary Science, v. 53, p. 5-49, doi: 10.1111/maps.12912.

Stöffler, D., Keil, K., and Scott, E.R.D., 1991, Shock metamorphism of ordinary chondrites: Geochimica et Cosmochimica Acta, v. 55, p. 3845-3867, doi: 10.1016/0016-7037(91)90078-J.

Tachibana, S., 2006, Chondrule formation and evolution of the early solar system: Journal of Mineralogical and Petrological Sciences, v. 101, p. 37-47.

Tachibana, S., and Huss, G.R., 2005, Sulfur isotope composition of putative primary troilite in chondrules from Bishunpur and Semarkona: Geochimica et Cosmochimica Acta, v. 69, p. 3075-3097, doi: 10.1016/j.gca.2004.06.025.

Tait, A.W., Fisher, K.R., and Simon, J.I., 2013, Strain measurements of chondrules and refraction inclusion in Allende: Lunar and Planetary Conference, v. 45, p. 1-2.

Tanaka, K.K., Tanaka, H., Nakazawa, K., and Nakagawa, Y., 1998, Shock heating due to accretion of a clumpy cloud onto a protoplanetary disk: Icarus, v. 134, p. 137-154, doi: 10.1006/icar.1998.5947.

Tielke, J.A., Zimmerman, M.E., and Kohlstedt, D.L., 2016, Direct shear of olivine single crystals: Earth and Planetary Science Letters, v. 455, p. 140-148, doi: 10.1016/j.epsl.2016.09.002.

Tielke, J.A., Zimmerman, M.E., and Kohlstedt, D.L., 2017, Hydrolytic weakening in olivine single crystals: Journal of Geophysical Research: Solid Earth, v. 122, p. 3465-3479, doi: 10.1002/2017JB014004.

Tomeoka, K., Yamahana, Y., and Sekine, T., 1999, Experimental shock metamorphism of the Murchison CM carbonaceous chondrite: Geochimica et Cosmochimica Acta, v. 63, p. 3683-3703, doi: 10.1016/S0016-7037(99)00149-0.

Tommasi, A., Mainprice, D., Canova, G., and Chastel, Y., 2000, Viscoplastic selfconsistent and equilibrium-based modeling of olivine lattice preferred orientations: Implications for the upper mantle seismic anisotropy: Journal of Geophysical Research, v. 105, p. 7893-7908, doi: 10.1029/1999JB900411.

Tsuchiyama, A., Osada, Y., Nakano, T., and Uesugi, K., 2004, Experimental reproduction of classic barred olivine chondrules: Open-system behavior of 
chondrule formation: Geochimica et Cosmochimica Acta, v. 68, p. 653-672, doi: 10.1016/S0016-7037(03)00448-4.

Villeneuve, J., Chaussidon, M., and Libourel, G., 2009, Evidence for 26Al homogeneous distribution in the solar system from the $\mathrm{Mg}$ isotopic composition of chondrules: Science, v. 325, p. 985-988, doi: 10.1126/science.1173907.

Villeneuve, J., Libourel, G., and Soulié, C., 2015, Relationships between type I and type II chondrules: Implications on chondrule formation processes: Geochimica et Cosmochimica Acta, v. 160, p. 277-305, doi: 10.1016/j.gca.2015.03.033.

Wadhwa, M., Amelin, Y., Davis, A.M., Lugmair, G.W., Meyer, B., Gounelle, M., and Desch, S.J., 2007, From dust to planetesimals: Implications for the solar protoplanetary disk from short-lived radionuclides, in Protostars and Planets V, p. 835-848.

Wasson, J.T., 2004, Petrographic evidence for rapid heating and cooling during chondrule formation, in Workshop on Chondrites and Protoplanetary Disk,.

Wasson, J.T., and Rubin, A.E., 2003, Ubiquitous low-FeO relict grains in type II chondrules and limited overgrowths on phenocrysts following the final melting event: Geochimica et Cosmochimica Acta, v. 67, p. 2239-2250, doi: 10.1016/S0016-7037(03)00023-1.

Watt, L.E., Bland, P.A., Prior, D.J., and Russell, S.S., 2006, Fabric analysis of Allende matrix using EBSD: Meteoritics and Planetary Science, v. 41, p. 989-1001.

Weertman, J., and Weertman, J.R., 1992, Elementary Dislocation Theory: New York, Oxford University Press, 213 p.

Weidenschilling, S.J., Marzari, F., and Hood, L.L., 1998, The origin of chondrules at Jovian resonances: Science, v. 279, p. 681-684, doi: 10.1126/science.279.5351.681.

Weinbruch, S., Büttner, H., Holzheid, A., Rosenhauer, M., and Hewins, R.H., 1998, On the lower limit of chondrule cooling rates: The significance of iron loss in dynamic crystallization experiments: Meteoritics and Planetary Science, v. 33, p. 65-74, doi: 10.1111/j.1945-5100.1998.tb01608.x.

Weisberg, M.K., McCoy, T.J., and Krot, A.N., 2006, Systematics and evaluation of meteorite classification, in Lauretta, D.S. and McSween, H.Y.J. eds., Meteorites and the Early Solar System II, Tuscon, University of Arizona Press, p. 19-52.

Weisberg, M.K., Smith, C., Benedix, G., Herd, C.D.K., Righter, K., Haack, H., Yamaguchi, A., Chennaoui Aoudjehane, H., and Grossman, J.N., 2010, The Meteoritical Bulletin, No. 97: Meteoritics and Planetary Science, v. 45, p. 449-493, doi: 10.1111/j.1945-5100.2010.01036.x.

Whattam, S.A., Hewins, R.H., Cohen, B.A., Seaton, N.C., and Prior, D.J., 2008, Granoblastic olivine aggregates in magnesian chondrules: Planetesimal fragments or thermally annealed solar nebula condensates? Earth and Planetary Science Letters, v. 269, p. 200-211, doi: 10.1016/j.epsl.2008.02.013.

Winston, E., McBreen, S., McBreen, B., and Hanlon, L., 2006, Gamma-ray bursts and giant lightning discharges in protoplanetary systems, in AIP Conference Proceedings, v. 836, p. 161-164, doi: 10.1063/1.2207880.

Wood, J.A., 1988, Chondritic meteorites and the solar nebula: Annual Review of Earth and Planetary Sciences, v. 16, p. 53-72. 
Yin, Q.-Z., Jacobsen, B., Moynier, F., and Hutcheon, I.D., 2007, Toward consistent chronology in the early solar system: High-resolution $53 \mathrm{Mn}-53 \mathrm{Cr}$ chronometry for chondrules: Astrophysical Journal, v. 662, p. L43-L46, doi: 10.1086/519282.

Yu, Y., Hewins, R.H., Alexander, C.M.O.D., and Wang, J., 2003, Experimental study of evaporation and isotopic mass fractionation of potassium in silicate melts: Geochimica et Cosmochimica Acta, v. 67, p. 773-786, doi: 10.1016/S00167037(02)01176-6.

Yurimoto, H., and Wasson, J.T., 2002, Extremely rapid cooling of a carbonaceouschondrite chondrule containing very $16 \mathrm{O}$-rich olivine and a $26 \mathrm{Mg}$-excess:

Geochimica et Cosmochimica Acta, v. 66, p. 4355-4363, doi: 10.1016/S00167037(02)01218-8.

Zaefferer, S., 2011, A critical review of orientation microscopy in SEM and TEM: Crystal Research and Technology, v. 46, p. 607-628, doi: 10.1002/crat.201100125.

Zanda, B., 2004, Chondrules: Earth and Planetary Science Letters, v. 224, p. 1-17, doi: 10.1016/j.eps1.2004.05.005. 
Appendix: Shock Data Figures of Non-EBSD Analyzed Samples

Shock data figures for samples that were not selected for EBSD analysis are presented below and on the following pages.
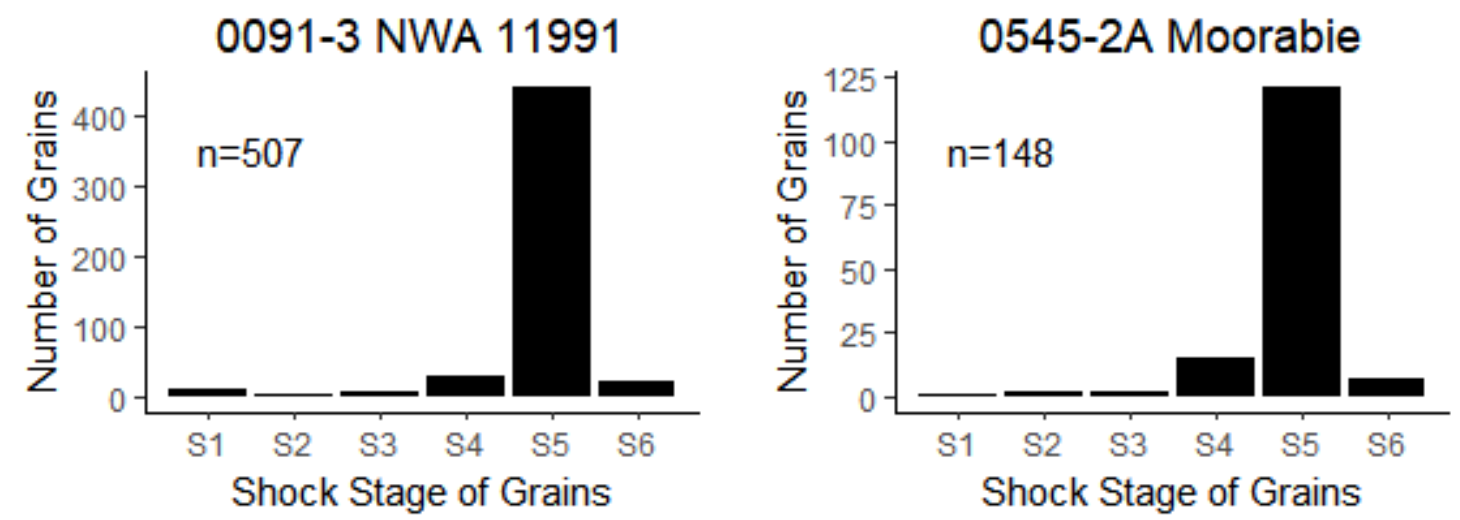

Figure 62. Grain Shock Stage Figure 63. Grain Shock Stage Histogram of Sample CML 0091-3. Histogram of Sample CML 0545-2A. Shock stage is as defined by Stöffler et Shock stage is as defined by Stöffler et al. (1991, 2018). al. (1991, 2018).
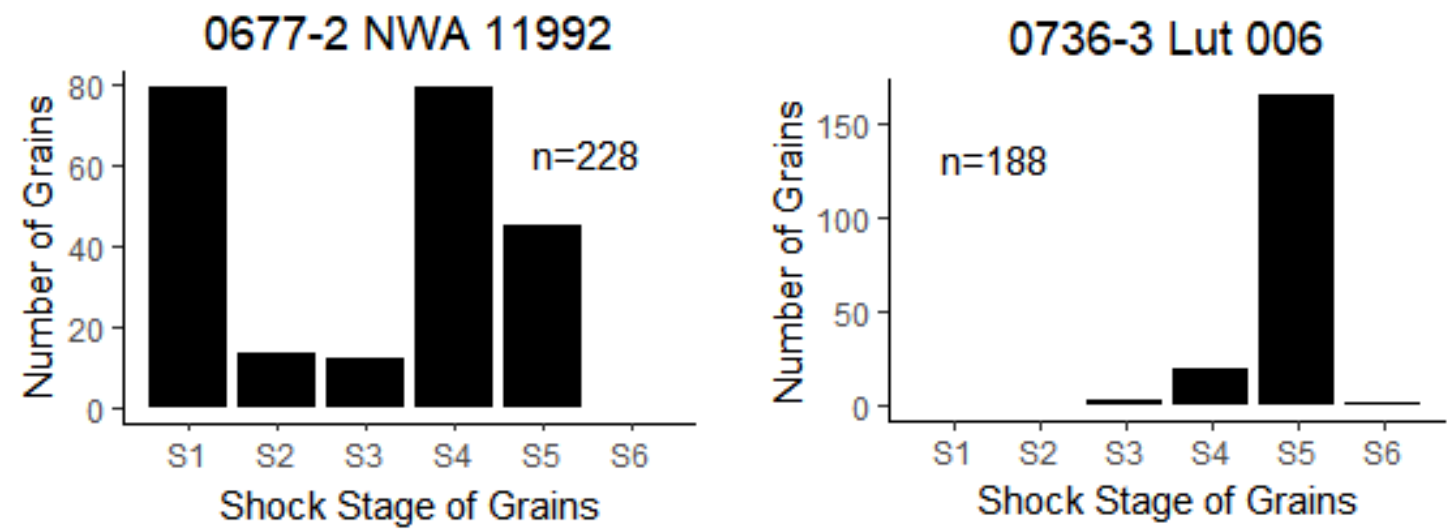

Figure 64. Grain Shock Stage Figure 65. Grain Shock Stage Histogram of Sample CML 0677-2. Histogram of Sample CML 0763-3. Shock stage is as defined by Stöffler et Shock stage is as defined by Stöffler et al. (1991, 2018).

al. (1991, 2018). 


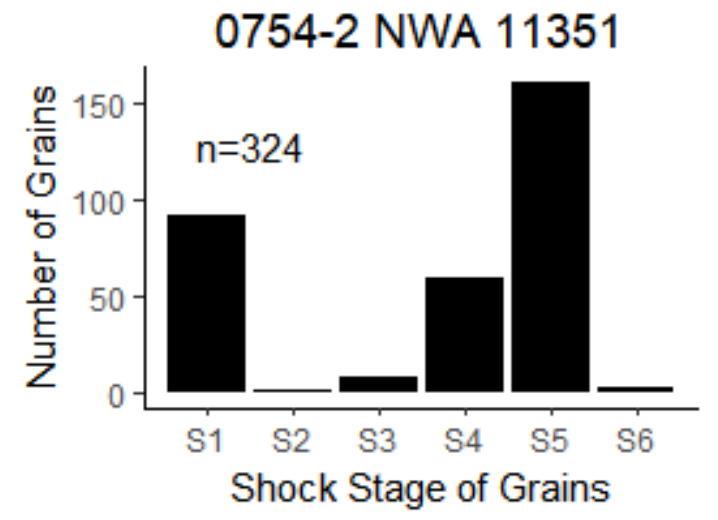

Figure 66. Grain Shock Stage Figure 67. Grain Shock Stage Histogram of Sample CML 0754-2. Histogram of Sample CML 0754-3. Shock stage is as defined by Stöffler et Shock stage is as defined by Stöffler et al. (1991, 2018). Sample is composed of different lithologies than 0754-3.

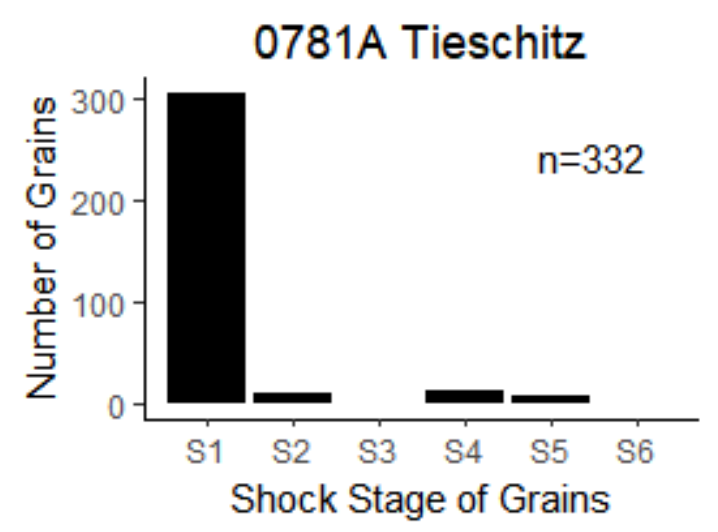

Figure 68. Grain Shock Stage Figure 69. Grain Shock Stage Histogram of Sample CML 0781A. Histogram of Sample CML 0856-2A. Shock stage is as defined by Stöffler et Shock stage is as defined by Stöffler et al. $(1991,2018)$.

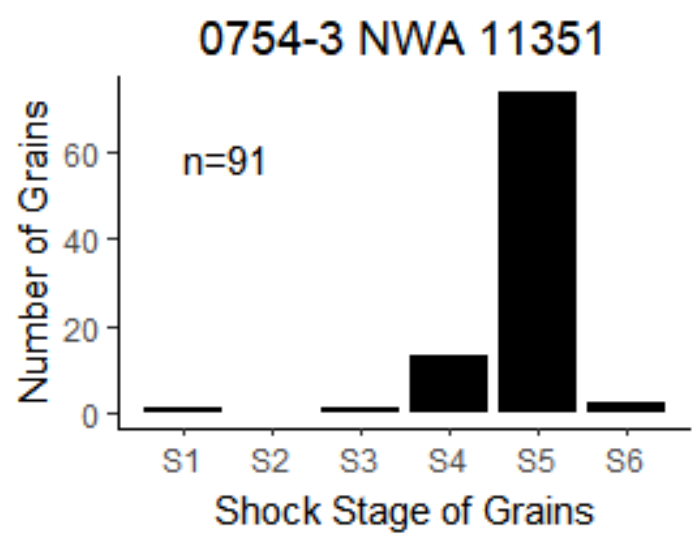
al. (1991, 2018). Sample is composed of a different lithology than 0754-2.

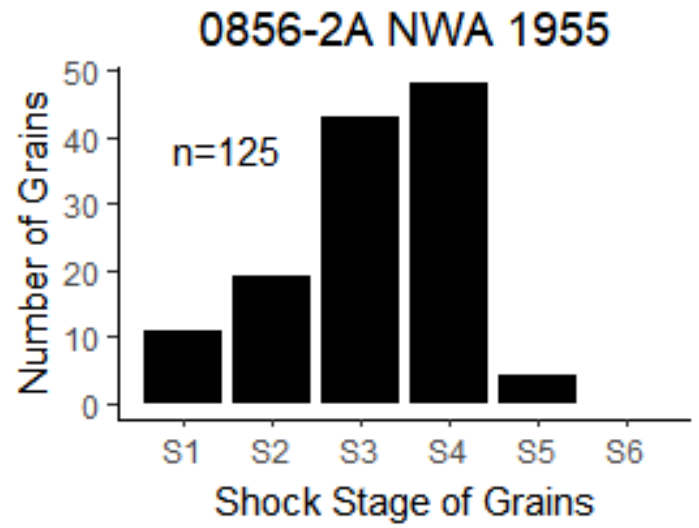

al. (1991, 2018). 

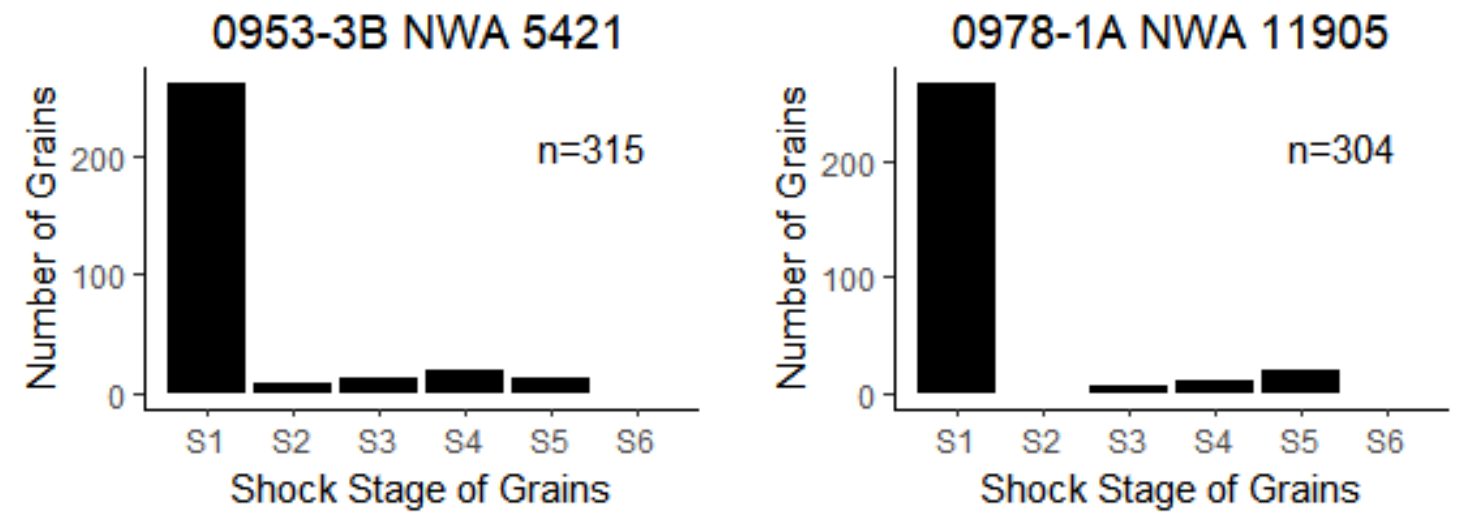

Figure 70. Grain Shock Stage Figure 71. Grain Shock Stage Histogram of Sample CML 0953-3B. Histogram of Sample CML 0978-1A. Shock stage is as defined by Stöffler et Shock stage is as defined by Stöffler et al. (1991, 2018).

al. (1991, 2018).
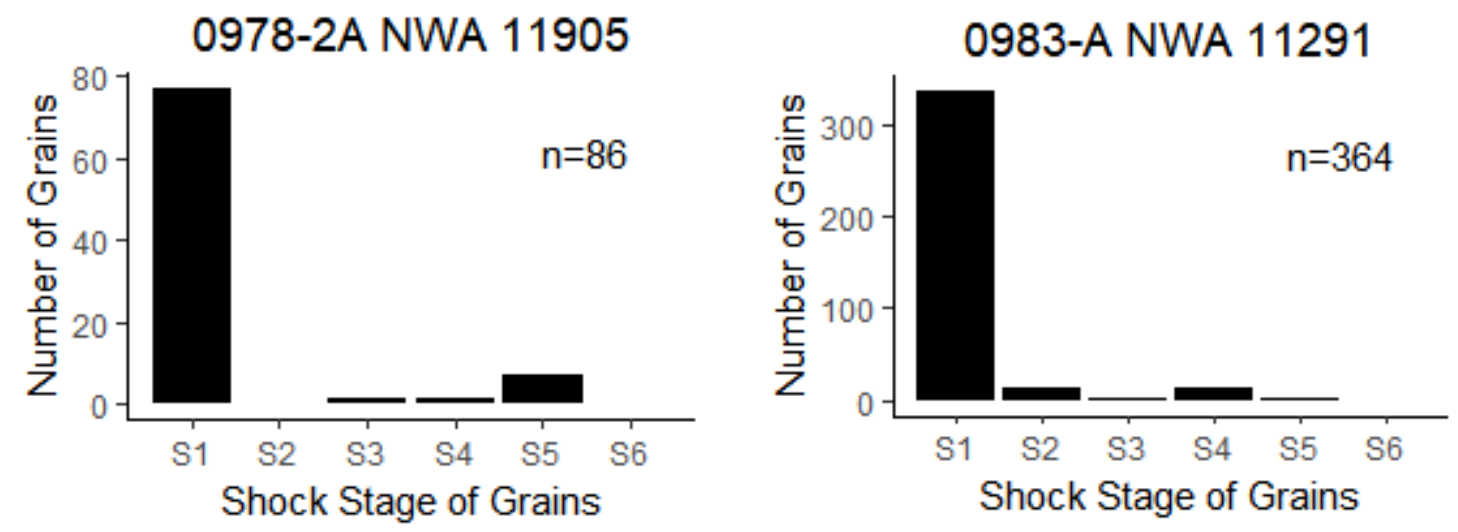

Figure 72. Grain Shock Stage Figure 73. Grain Shock Stage Histogram of Sample CML 0978-2A. Histogram of Sample CML 0983-A. Shock stage is as defined by Stöffler et Shock stage is as defined by Stöffler et al. al. (1991, 2018). (1991, 2018). 

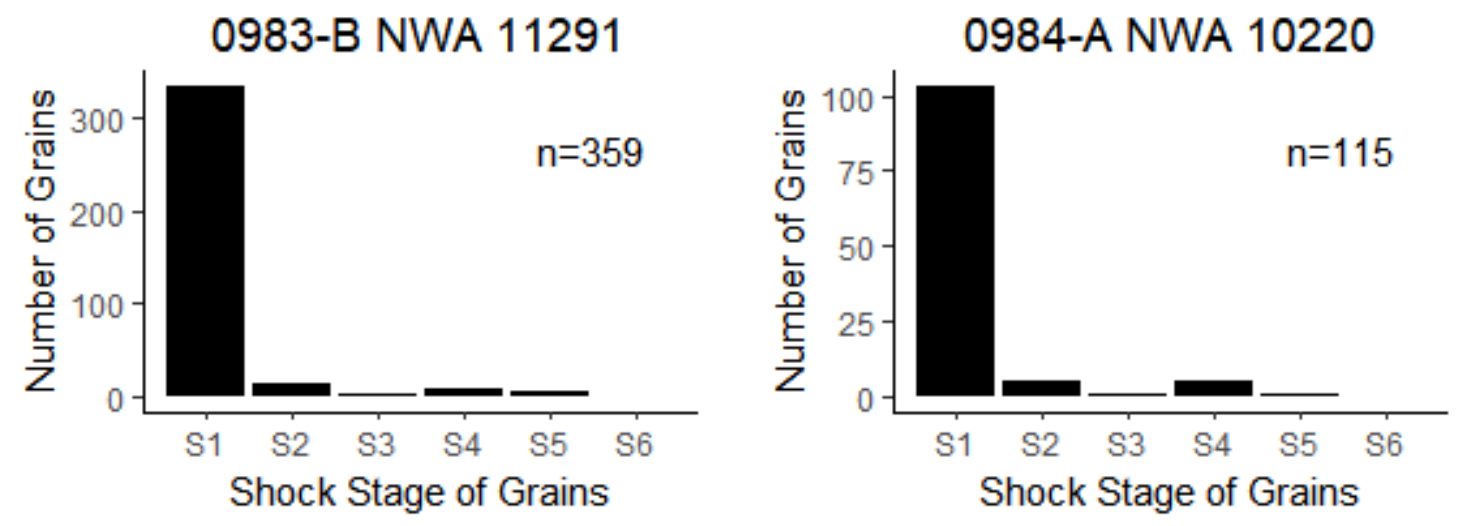

Figure 74. Grain Shock Stage Figure 75. Grain Shock Stage Histogram of Sample CML 0983-B. Histogram of Sample CML 0984-A. Shock stage is as defined by Stöffler et Shock stage is as defined by Stöffler et al. (1991, 2018).

al. (1991, 2018).

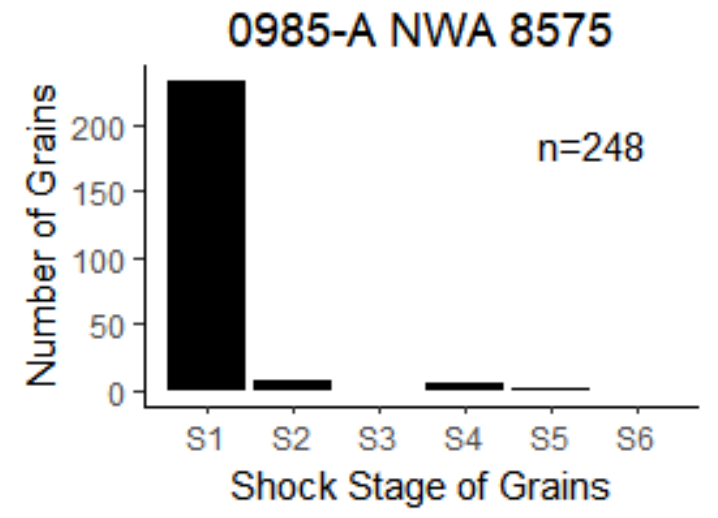

Figure 76. Grain Shock Stage Histogram of Sample CML 0985-A. Shock stage is as defined by Stöffler et al. (1991, 2018). 
0091-3 NWA 11991
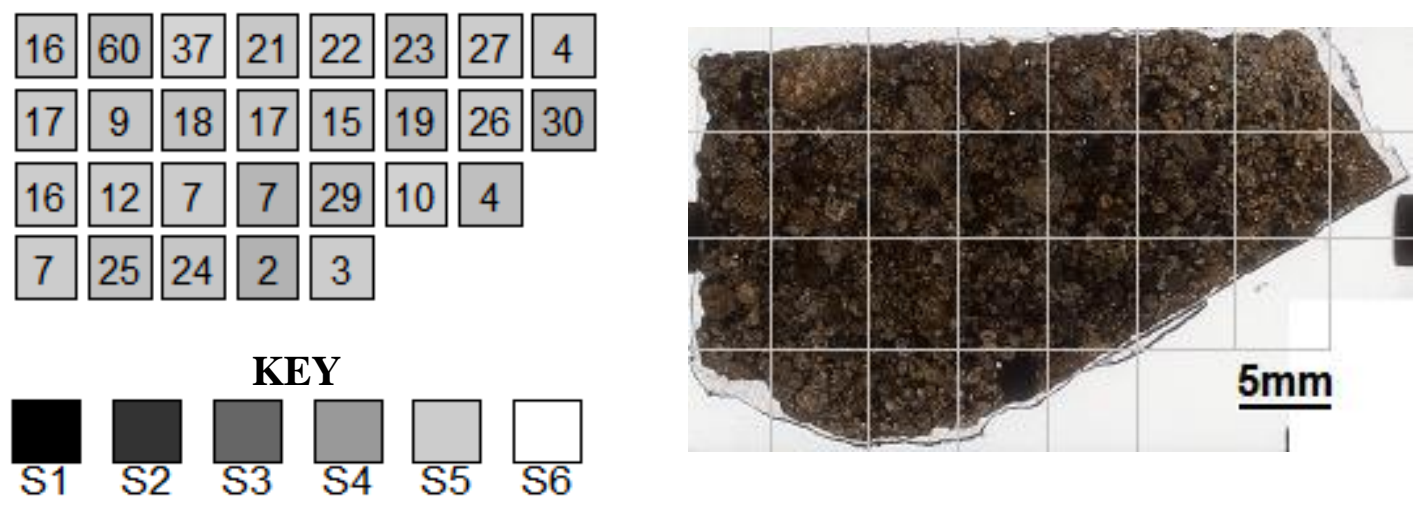

Figure 77. Shock Map of Sample CML 0091-3. Left image is a sectored shock map, and the right is an image of the sample in the same orientation. Numbers in the shock map sectors are the number of measured grains in those cells. Sector color is based on the weighted shock stage of a sector on the scale defined by the key.

\section{5-2A Moorabie}
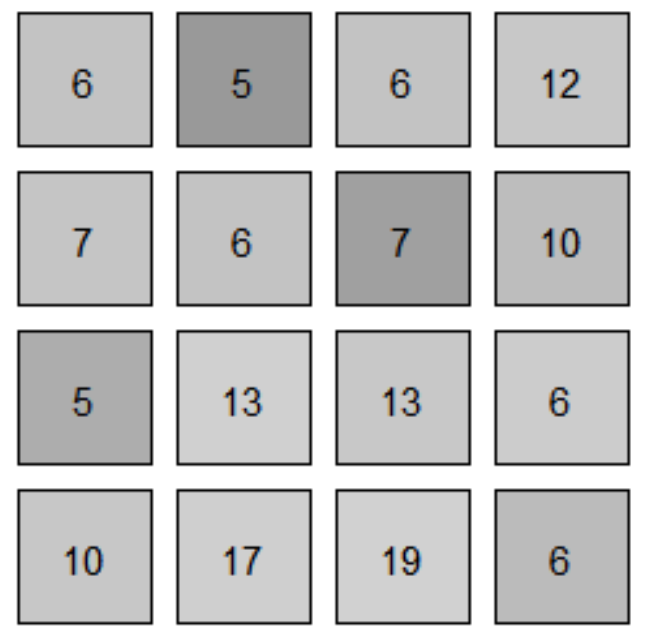

\section{KEY}
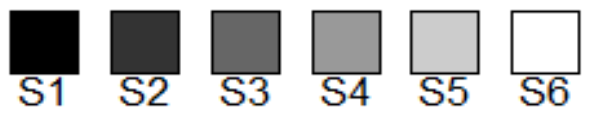

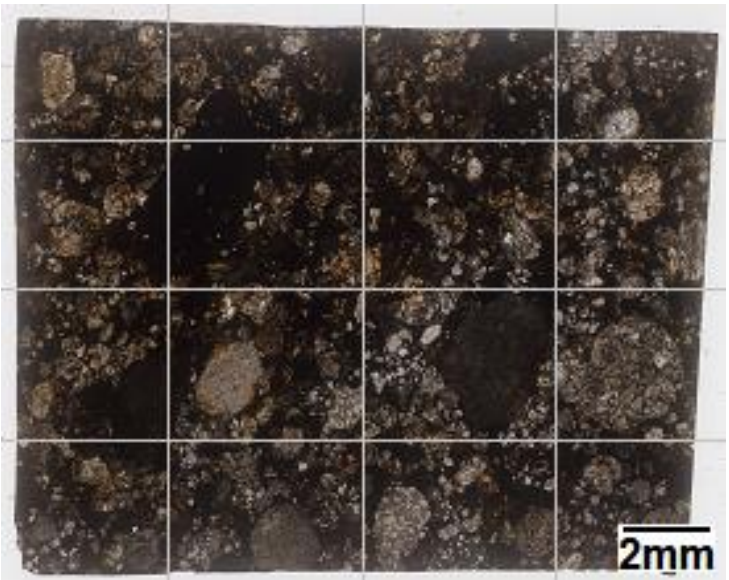

Figure 78. Shock Map of Sample CML 0545-2A. Left image is a sectored shock map, and the right is an image of the sample in the same orientation. Numbers in the shock map sectors are the number of measured grains in those cells. Sector color is based on the weighted shock stage of a sector on the scale defined by the key. 
0677-2 NWA 11992
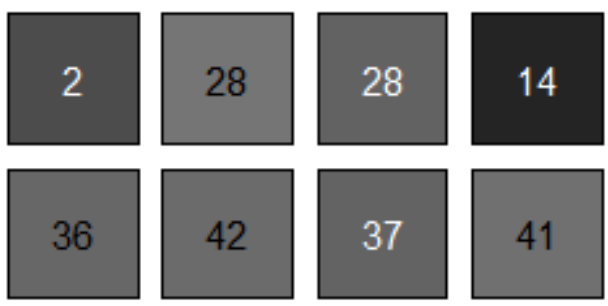

KEY
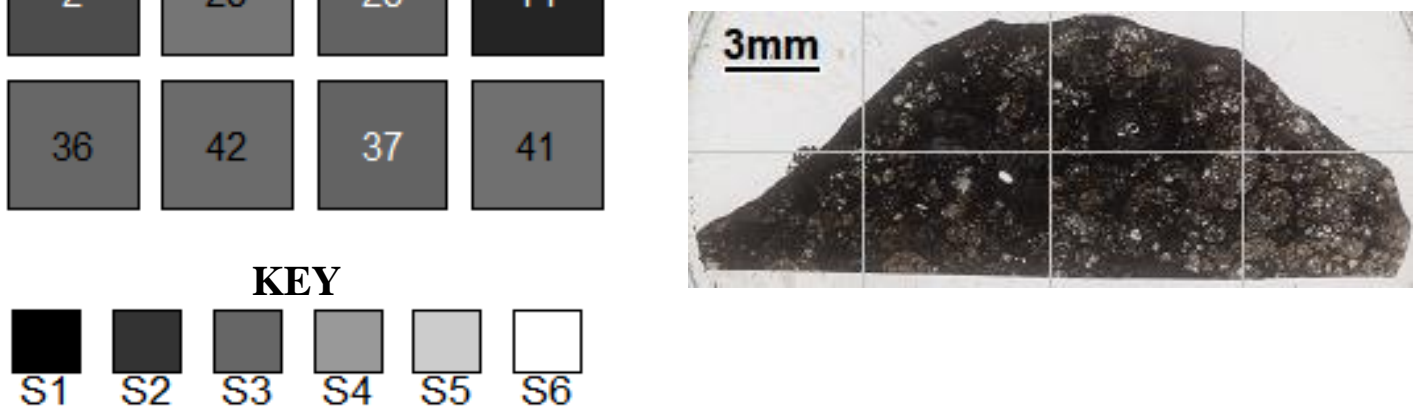

Figure 79. Shock Map of Sample CML 0677-2. Left image is a sectored shock map, and the right is an image of the sample in the same orientation. Numbers in the shock map sectors are the number of measured grains in those cells. Sector color is based on the weighted shock stage of a sector on the scale defined by the key.

\section{6-3 Lut 006}
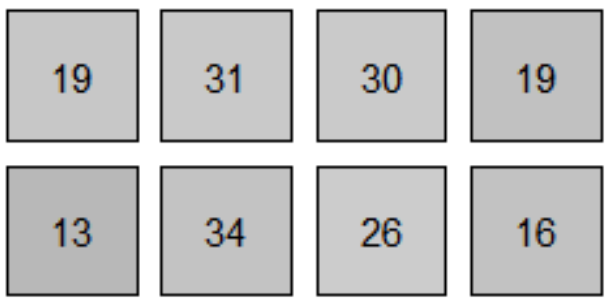

16
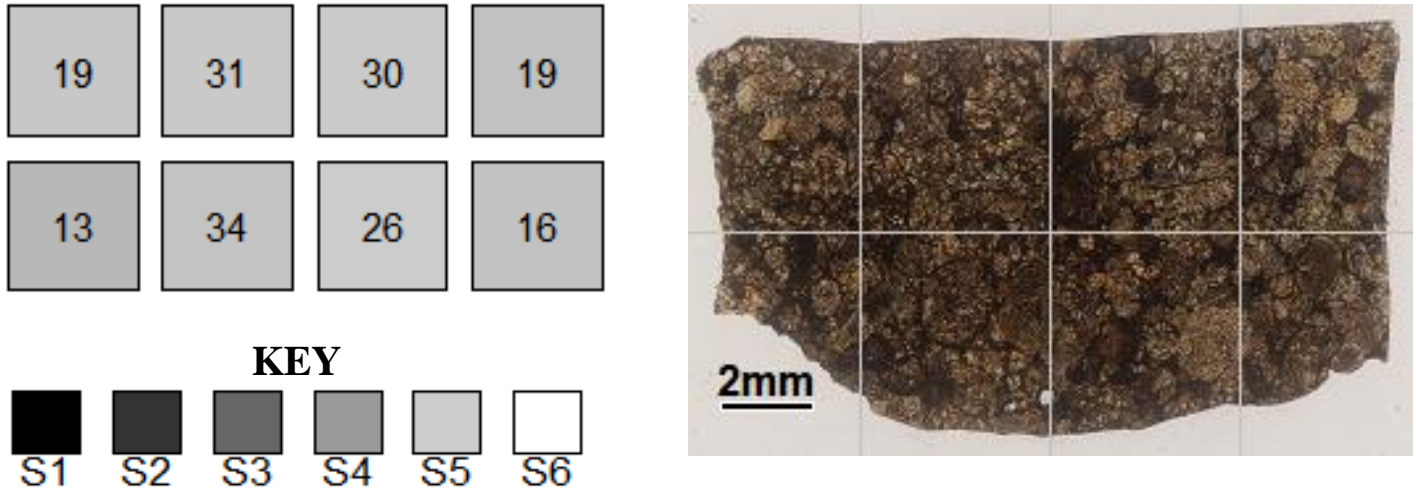

Figure 80. Shock Map of Sample CML 0736-3. Left image is a sectored shock map, and the right is an image of the sample in the same orientation. Numbers in the shock map sectors are the number of measured grains in those cells. Sector color is based on the weighted shock stage of a sector on the scale defined by the key. 
0754-2 NWA 11351
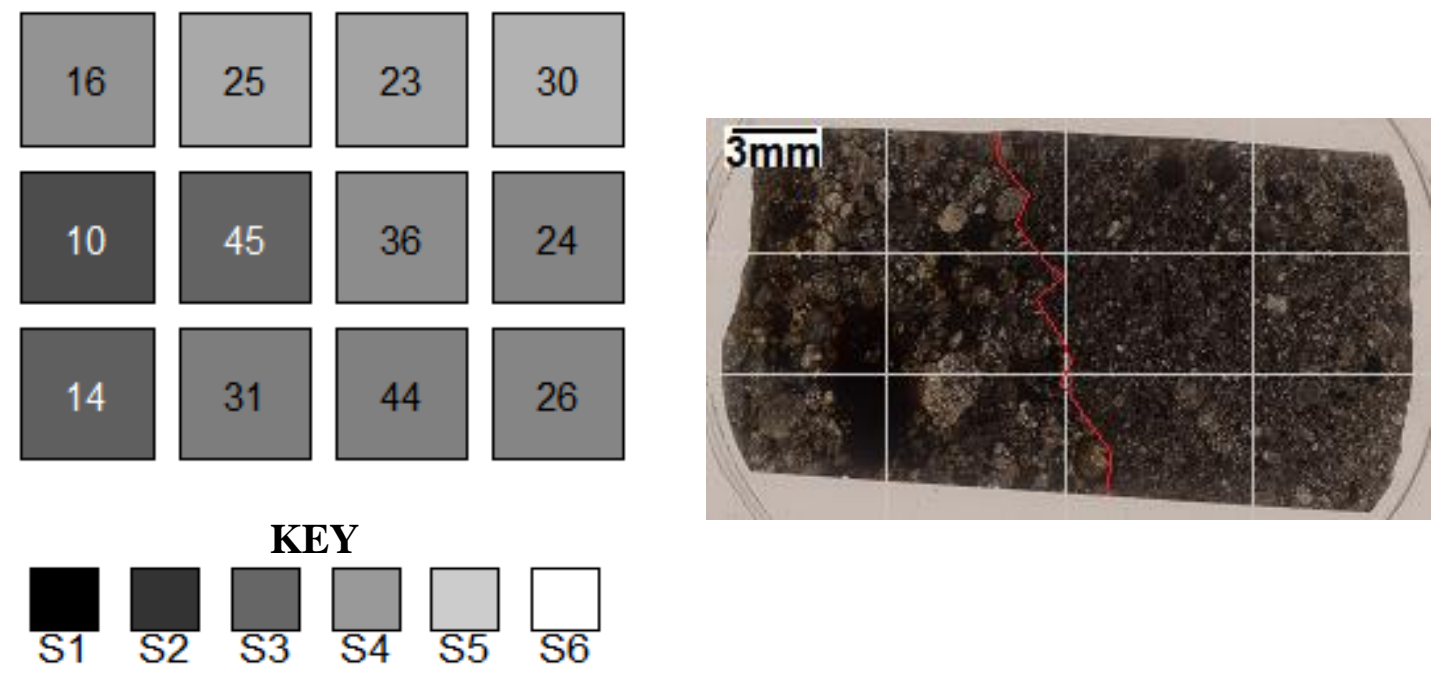

Figure 81. Shock Map of Sample CML 0754-2. Left image is a sectored shock map, and the right is an image of the sample in the same orientation. Numbers in the shock map sectors are the number of measured grains in those cells. Sector color is based on the weighted shock stage of a sector on the scale defined by the key. Red line denotes boundary of cluster chondrite clast, which occupies the left part of the thin-section image.

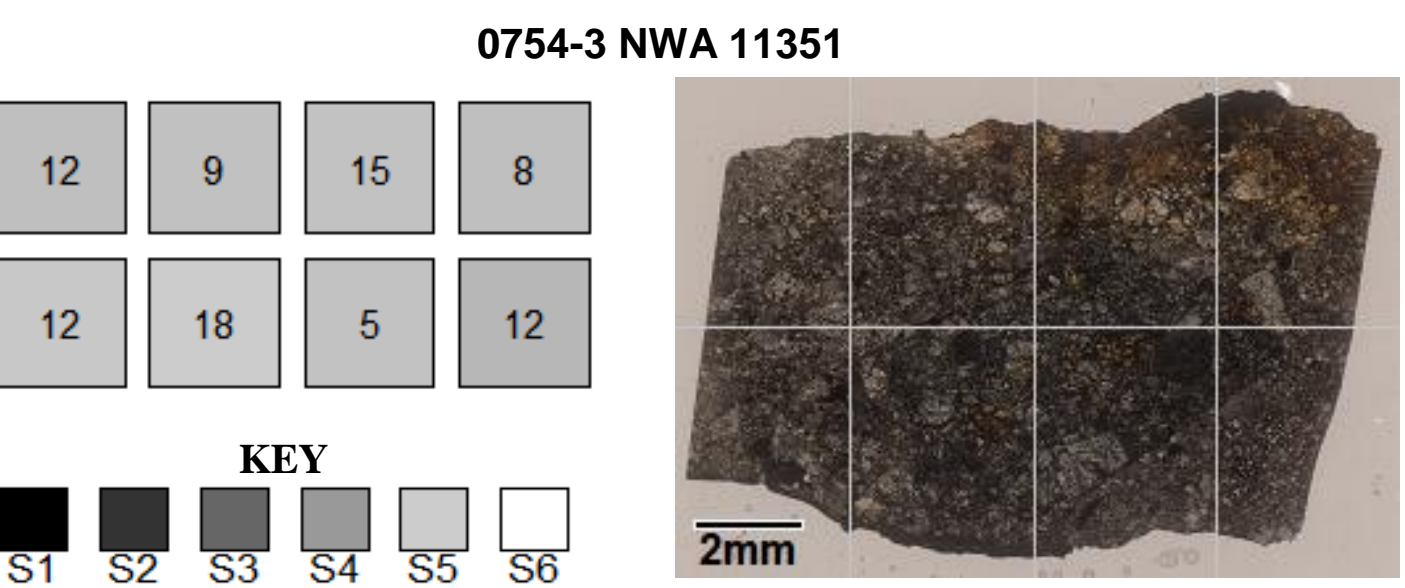

Figure 82. Shock Map of Sample CML 0754-3. Sample is composed of lithology C. Left image is a sectored shock map, and the right is an image of the sample in the same orientation. Numbers in the shock map sectors are the number of measured grains in those cells. Sector color is based on the weighted shock stage of a sector on the scale defined by the key. 


\section{A Tieschitz}
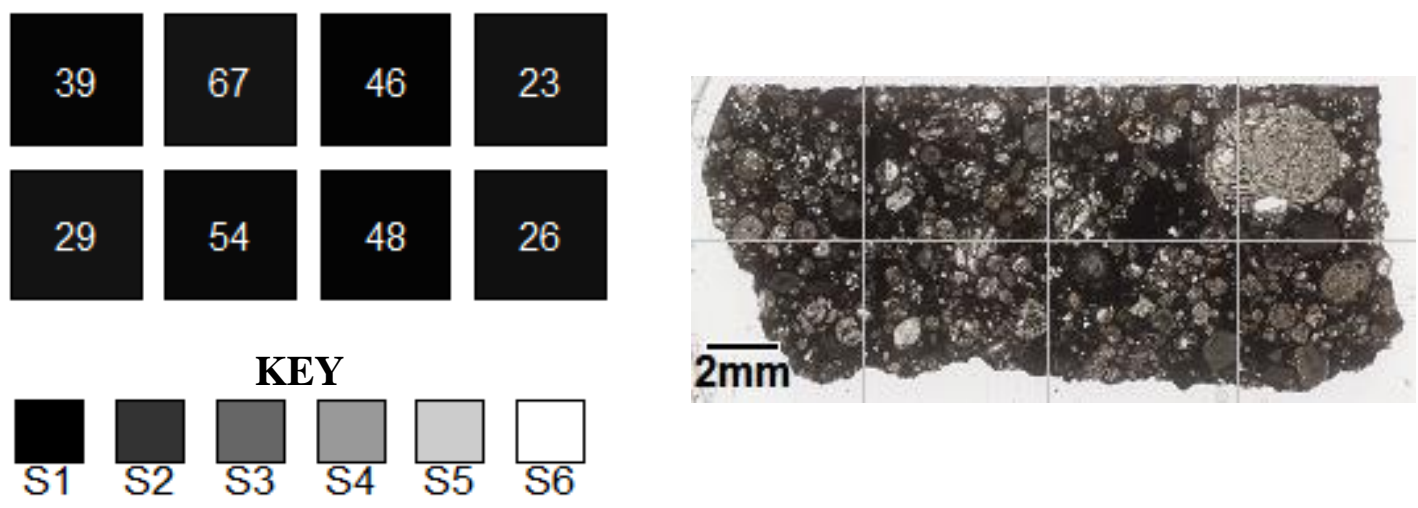

Figure 83. Shock Map of Sample CML 0781A. Left image is a sectored shock map, and the right is an image of the sample in the same orientation. Numbers in the shock map sectors are the number of measured grains in those cells. Sector color is based on the weighted shock stage of a sector on the scale defined by the key.

\section{6-2A NWA 1955}
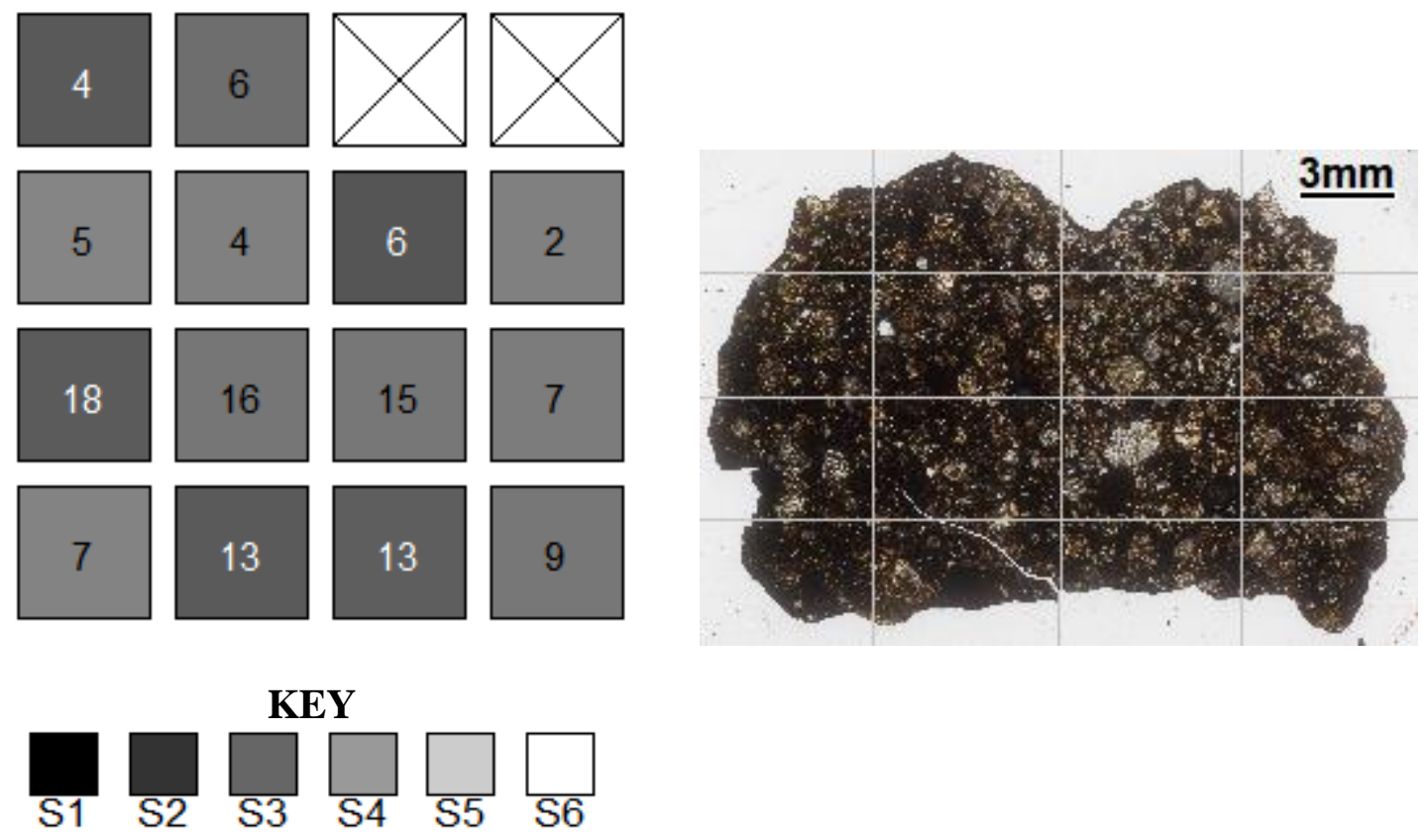

Figure 84. Shock Map of Sample CML 0856-2A. Left image is a sectored shock map, and the right is an image of the sample in the same orientation. Numbers in the shock map sectors are the number of measured grains in those cells. Sector color is based on the weighted shock stage of a sector on the scale defined by the key. Sectors with an $\mathrm{X}$ have no grains usable for shock stage assessment. 
0953-3B NWA 5421
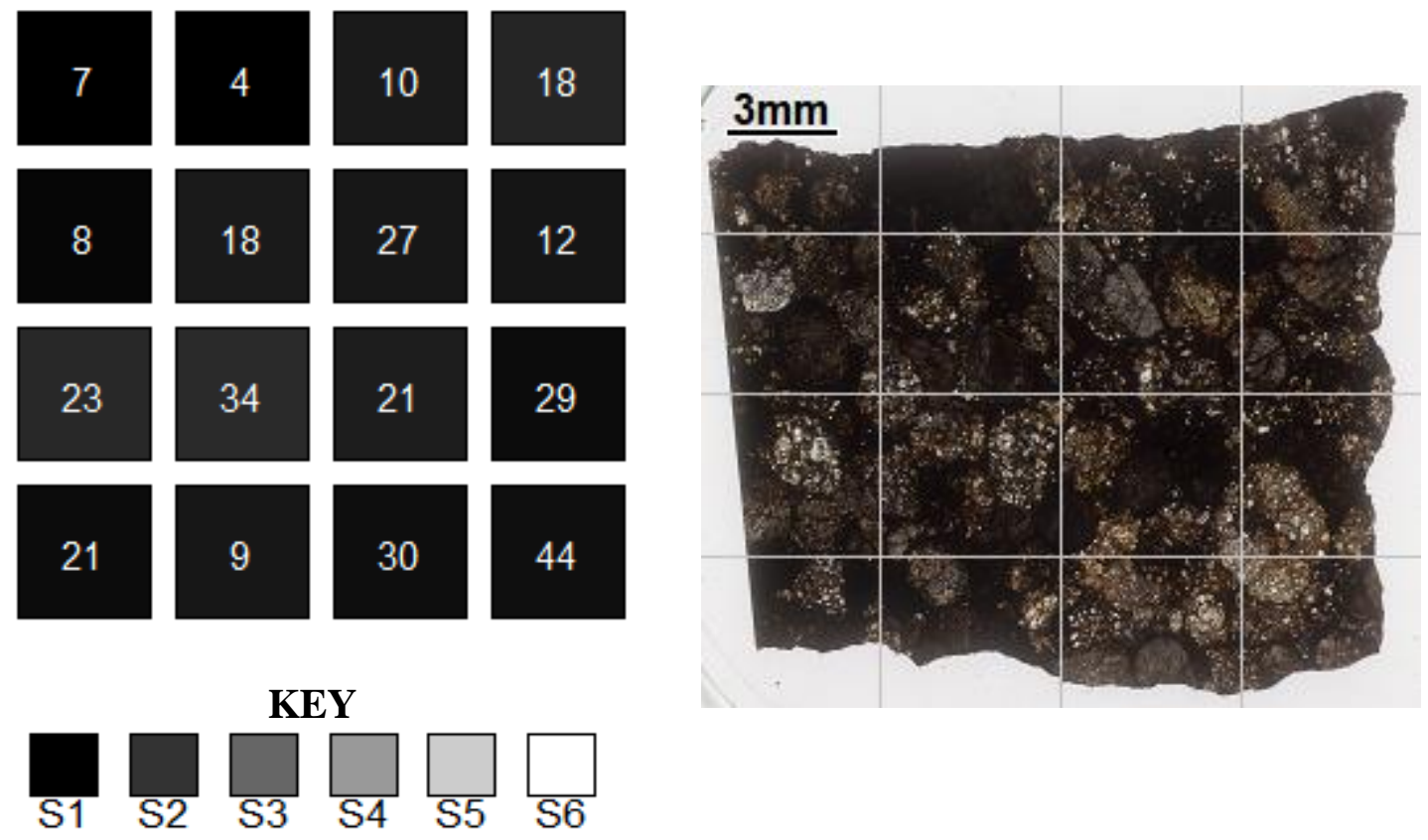

Figure 85. Shock Map of Sample CML 0953-3B. Left image is a sectored shock map, and the right is an image of the sample in the same orientation. Numbers in the shock map sectors are the number of measured grains in those cells. Sector color is based on the weighted shock stage of a sector on the scale defined by the key.

0978-1A NWA 11905
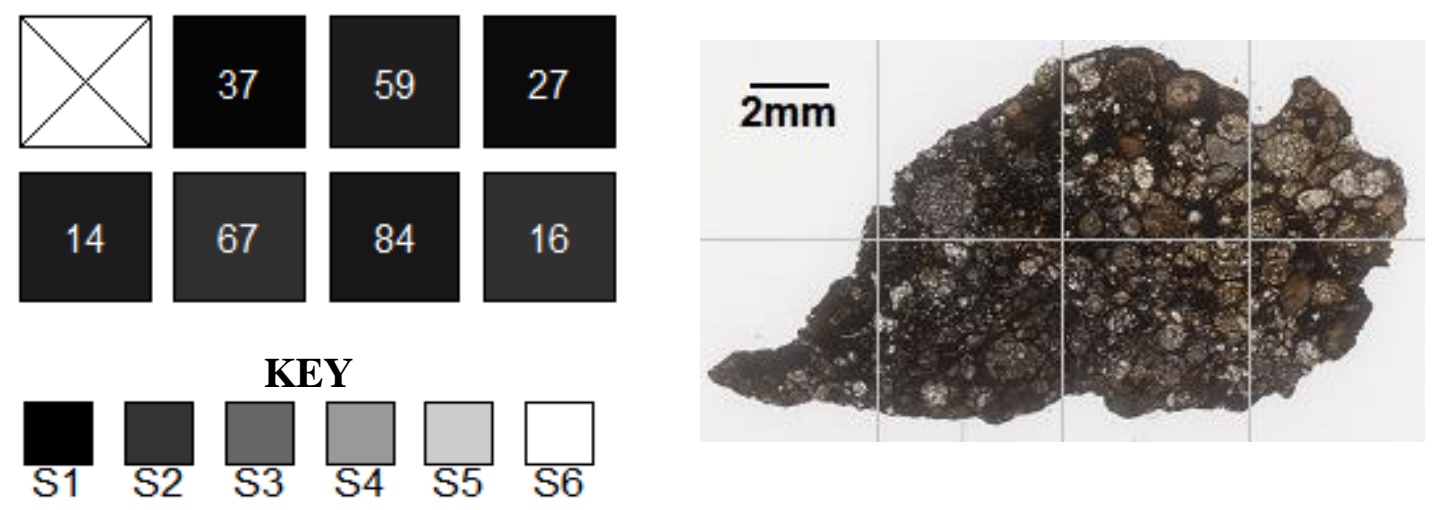

\section{6}
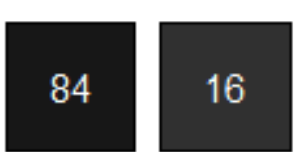

Figure 86. Shock Map of Sample CML 0978-1A. Left image is a sectored shock map, and the right is an image of the sample in the same orientation. Numbers in the shock map sectors are the number of measured grains in those cells. Sector color is based on the weighted shock stage of a sector on the scale defined by the key. 
0978-2A NWA 11905
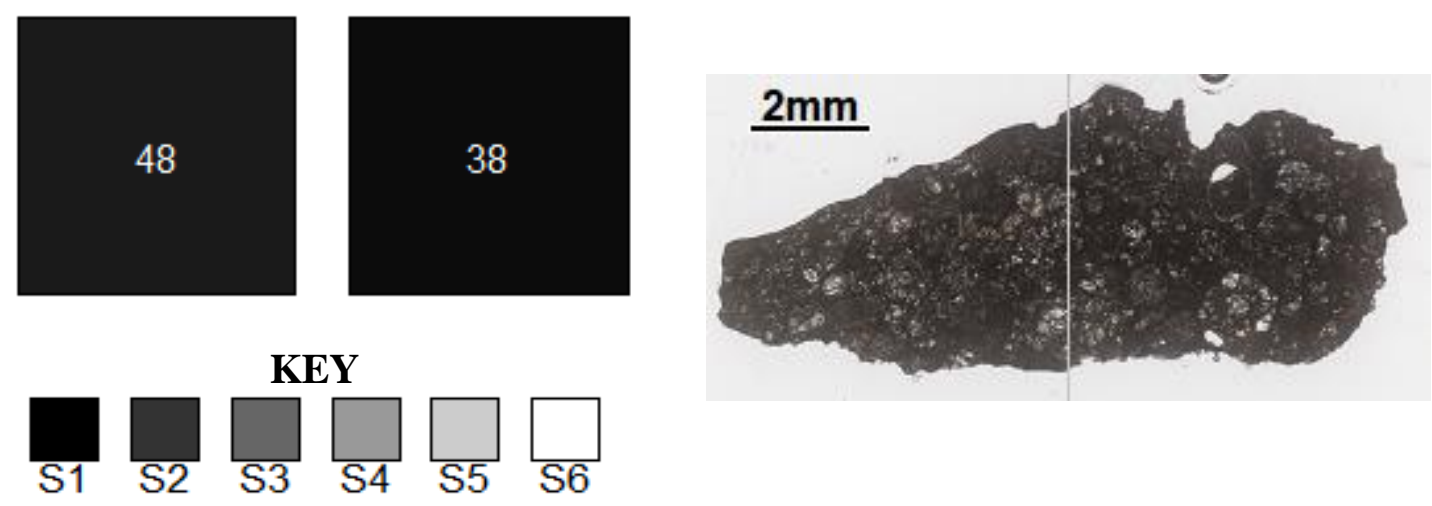

Figure 87. Shock Map of Sample CML 0978-2A. Left image is a sectored shock map, and the right is an image of the sample in the same orientation. Numbers in the shock map sectors are the number of measured grains in those cells. Sector color is based on the weighted shock stage of a sector on the scale defined by the key.

\section{3-A NWA 11291}
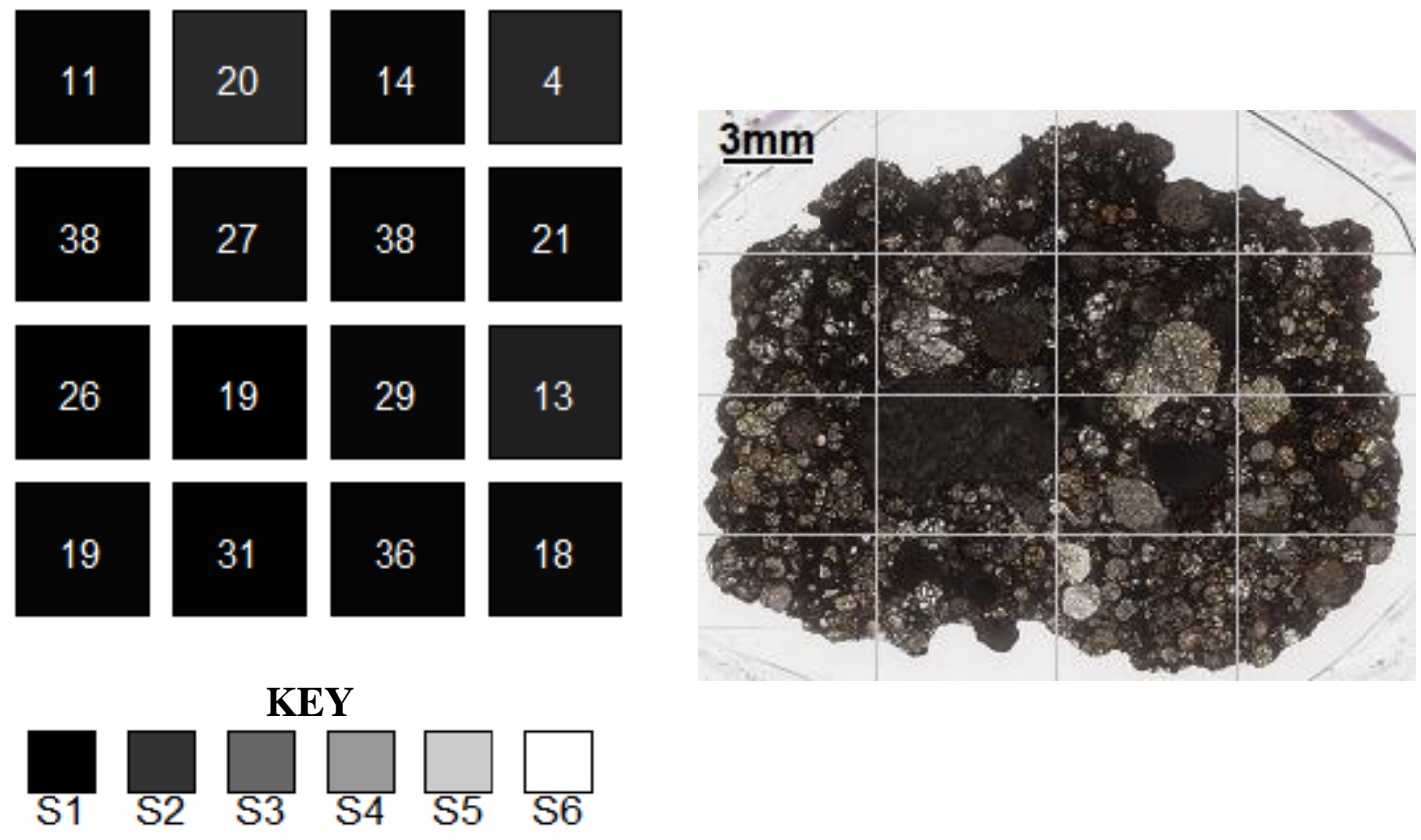

Figure 88. Shock Map of Sample CML 0983-A. Left image is a sectored shock map, and the right is an image of the sample in the same orientation. Numbers in the shock map sectors are the number of measured grains in those cells. Sector color is based on the weighted shock stage of a sector on the scale defined by the key. 
0983-B NWA 11291
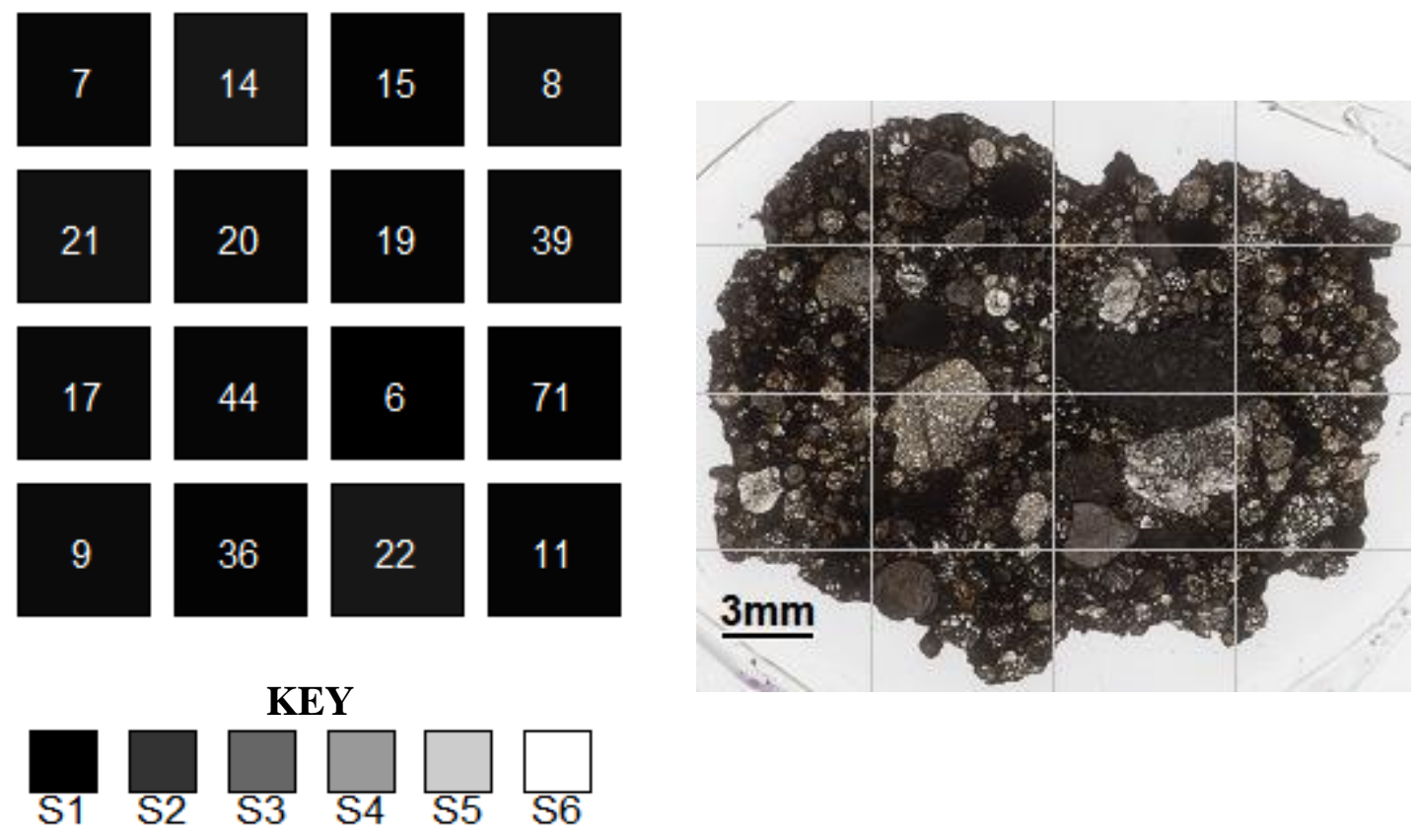

Figure 89. Shock Map of Sample CML 0983-B. Left image is a sectored shock map, and the right is an image of the sample in the same orientation. Numbers in the shock map sectors are the number of measured grains in those cells. Sector color is based on the weighted shock stage of a sector on the scale defined by the key. 
0984-A NWA 10220
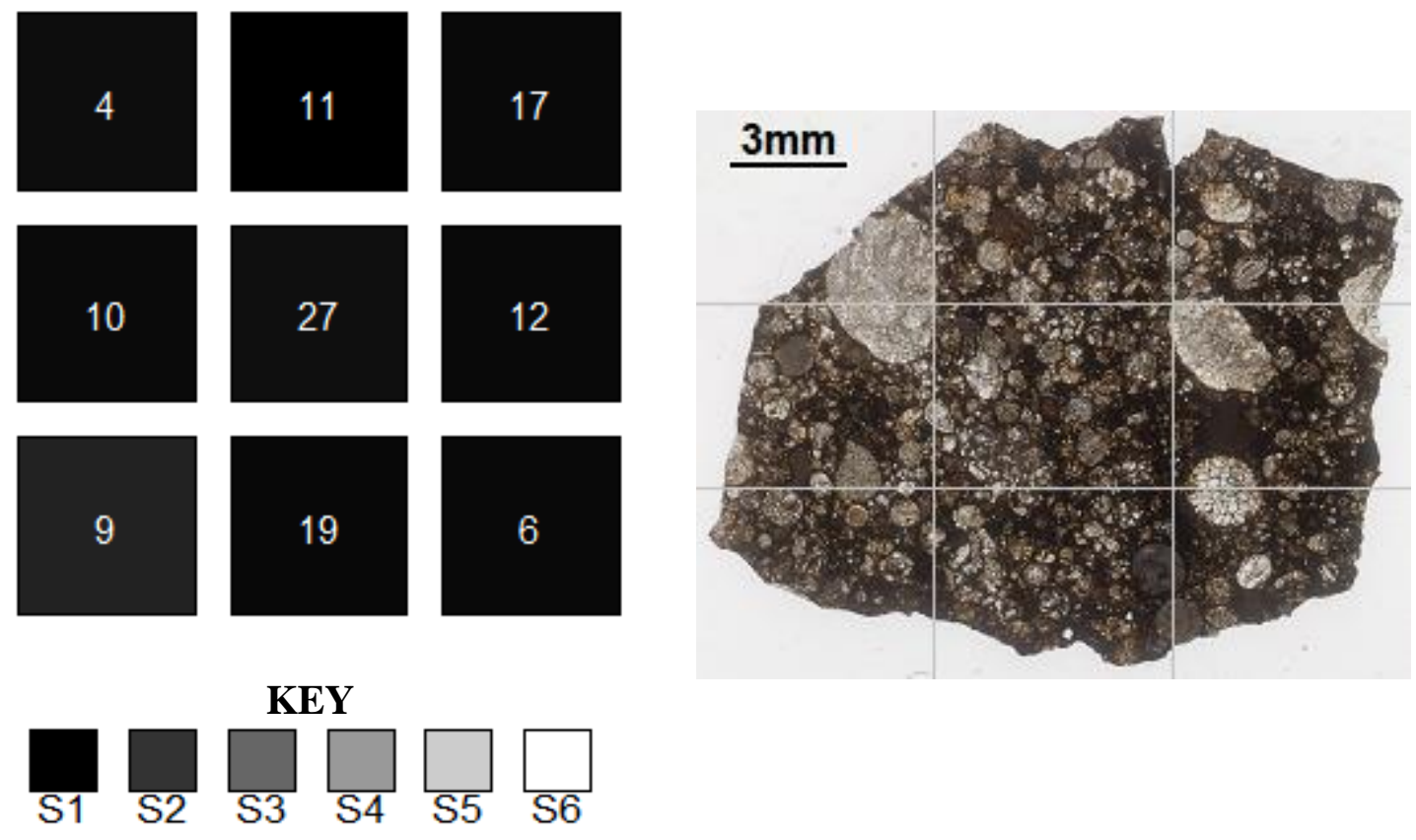

Figure 90. Shock Map of Sample CML 0984-A. Left image is a sectored shock map, and the right is an image of the sample in the same orientation. Numbers in the shock map sectors are the number of measured grains in those cells. Sector color is based on the weighted shock stage of a sector on the scale defined by the key. 


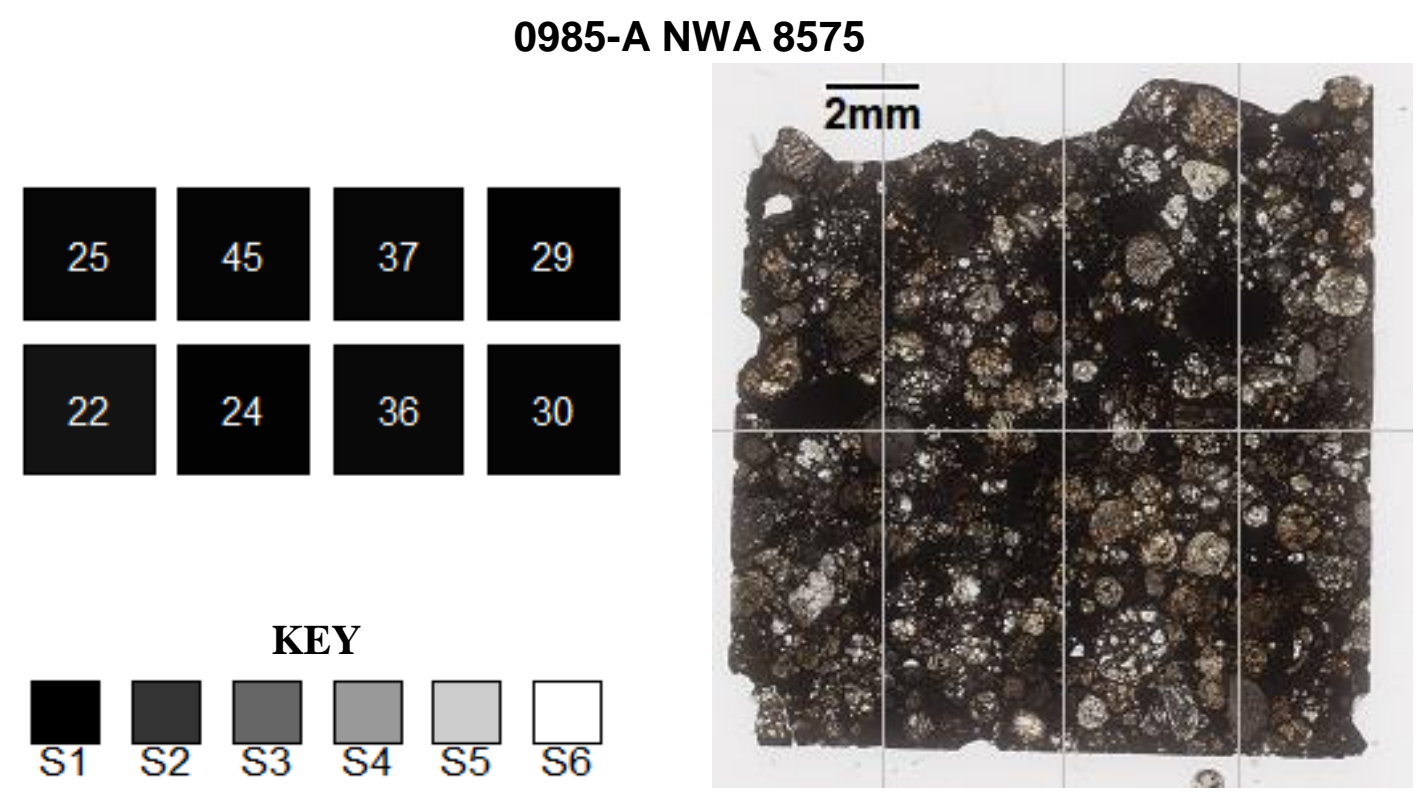

Figure 91. Shock Map of Sample CML 0985-A. Left image is a sectored shock map, and the right is an image of the sample in the same orientation. Numbers in the shock map sectors are the number of measured grains in those cells. Sector color is based on the weighted shock stage of a sector on the scale defined by the key. 